\title{
The 7th International Conference on Ambulatory Monitoring of Physical Activity and Movement
}

We are pleased to present the first special issue of the Journal for the Measurement of Physical Behaviour. This special issue features, for the first time, the abstracts of all the posters that were presented at the $7^{\text {th }}$ International Conference on Ambulatory Monitoring of Physical Activity and Movement (ICAMPAM).

The ICAMPAM conferences are hosted biannually by the International Society for the Measurement of Physical Behaviour (ISMPB). Our society is a non-profit scientific society that focuses on issues related to ambulatory monitoring, wearable monitors, movement sensors, physical activity, sedentary behaviour, movement behaviour, body postures, sleep, and constructs related to physical behaviours. Therefore, the published abstracts of this special issue focus on the objective measurement and quantification of physical behaviours such as:

- all free-living physical behaviours (including sleep) in its different forms (volumes and patterns which could give an indication of quality)

- measurements that are unrestricted, prolonged, and unsupervised

- measurements of physiological responses (e.g., energy expenditure) that are directly related to physical behaviours

- a wide range of applications: clinical, public health, behaviour sciences, etc.

The ICAMPAM conferences are designed to provide a forum for researchers and students to discuss the latest developments in physical behaviour monitoring using wearable devices. This first virtual conference, like all of the previous in-person meetings, served as a meeting point for young scientists and renowned experts in the field of health sciences, engineering, medical sciences, physiology, psychology, sports sciences, and more. The organising committee paid special attention to create a conference programme where many young scientists had the opportunity to present their work. The virtual format featured 20 invited speakers who gave live or prerecorded talks on a wide range of topics, including a major collaborative venture between pharma and academia, a DEI session, and a lively debate. The keynote presentations will be available to ISMPB members via our website.

We hope that the contents of this special issue will be informative to our readers and to new readers interested in objective measurement of physical behaviours.

On behalf of the organizing committee,

Jeff Hausdorff \& Martina Mancini, ICAMPAM 2021 Co-chairs

Malcolm Granat, ISMPB President

\section{ICAMPAM Conference Abstracts}

\section{Applications: Clinical Populations}

Association Between Physical Activity and Sleep Quality Among Hospitalized Older Adults With Dementia

Ashley Kuzmik ${ }^{1}$, Marie Boltz ${ }^{1}$

${ }^{1}$ The Pennsylvania State University
Introduction: Hospitalized older persons with dementia are at increased risk of poor sleep quality, associated with worse health outcomes, including cardiometabolic derangements and delirium. Physical activity has been associated with better sleep in non-acute care settings. The aim of this study was to assess the association between daytime physical activity and sleep quality in hospitalized older persons with dementia. Methods: This was a secondary analysis of an ongoing cluster randomized controlled trial. The sample included 259 participants diagnosed with dementia. Daytime physical activity and sleep quality were measured using the MotionWatch 8 (MW8). Sleep quality was defined by four characteristics: sleep duration, sleep efficiency, sleep latency, and sleep fragmentation. Linear regressions were conducted to test the associations between physical activity and sleep quality. Results: Most participants were either non-Hispanic Black (47.9\%) or non-Hispanic White $(49.8 \%)$, female $(60.2 \%)$, with a mean age of $81.36 \pm 8.24$, and indicated significant cognitive impairment $(\mathrm{MoCA}=$ $10.73 \pm 6.94)$. Higher levels of daytime physical activity were significantly associated with sleep duration $(\beta=.17, \mathrm{t}=2.8(253), \mathrm{p}=.006)$, sleep efficiency $(\beta=.18, \mathrm{t}=3.0(253), \mathrm{p}=.003)$, and sleep fragmentation $(\beta=-.23, t=-3.74(253), p<.001)$, but not sleep latency $(\beta=-.05$, $\mathrm{t}=-.78, \mathrm{p}=.435)$. All models were adjusted for age, gender, race, and comorbidities. Discussion: Findings suggest that daytime physical activity may increase sleep duration, improve sleep efficiency, and decrease sleep fragmentation among hospitalized older persons with dementia. This study highlights the need to promote daytime physical activity to improve sleep quality among older persons with dementia during hospitalization.

Automated Event-Based Algorithm for Quantifying Daily Life Ischemic Events in Peripheral Artery Disease

Aline Taoum ${ }^{1}$, Pierre Jéhannin ${ }^{2}$, Ségolène Chaudru ${ }^{3}$, Pierre-Yves de Müllenheim ${ }^{4}$, Guillaume Mahé $^{2}$, Alexis Le Faucheur ${ }^{1}$

${ }^{1}$ University of Rennes, ${ }^{2}$ University Hospital, ${ }^{3}$ University of Rennes 1, ${ }^{4}$ Institute of Physical Education and Sport Sciences (IFEPSA), UCO

Introduction: This study aimed to compare different algorithms in the implementation of an event-based analysis method of the daily walking pattern using wearable monitors to quantify walking pain manifestation (WPM) and stop-induced by walking pain (SIWP) in people with lower extremity peripheral artery disease (PAD). Methods: During a 7-day freeliving period, $21 \mathrm{PAD}$ participants wore a wGT3X+ accelerometer and a watch on which they were asked to press an event marker button to record WPM and SIWP. We compared different algorithms of first removing short $(\leq 15 \mathrm{~s})$ walking or stopping bouts and assigning SIWP within $15 \mathrm{~s}$ or 60 s after the end of bouts. Then, the daily pain-free walking time and maximal walking time were computed. Results: Figure 1 shows that removing short walking bouts first yielded lower WPM/week and higher SIWP/week compared to our original algorithm and to removing short stopping bouts first $(\mathrm{P}<0.05)$. Consequently, it provided significantly lower pain-free and maximal walking times $(\mathrm{P}<0.05)$ than both algorithms. It also provided higher maximal pain-free walking time than Gardner and Strandness tests and higher maximal walking time than Strandness test only $(\mathrm{P}<0.05)$. Discussion: When implementing an 
event-based algorithm to detect intermittent daily walking bouts, removing short bouts first of either stopping or walking significantly impacted clinical estimation of walking capacity in PAD. Although there was no direct observation of actual ischemic events, we consider that removing short walking bouts first would provide the most consistent assessment of walking capacity because this algorithm significantly increased the allocation of ischemic events, had a more consistent difference between the mean and the maximal pain-free and maximal walking times, and previously provided better detection of walking bouts in outdoor walking sessions. This automated event-based method will be implemented on our free-access web platform (mapam.ens-rennes.fr).

\section{Changes in the Activity Composition Over a Period of Two Years in People With Type 2 Diabetes and Prediabetes}

Kristina Larsson ${ }^{1}$, Philip von Rosen ${ }^{2}$, Jenny Rossen ${ }^{1}$, Unn-Britt Johansson $^{1}$, Maria Hagströmer ${ }^{2}$

${ }^{1}$ Sophiahemmet University, ${ }^{2}$ Karolinska Institutet

Introduction: The aim was to describe changes in activity composition (AC) over a period of two years, based on baseline physical activity (PA) level, in people with type 2 diabetes and prediabetes participating in a two-year PA intervention. Methods: Longitudinal data on 118 participants with type 2 diabetes and prediabetes ( $43 \%$ female, mean age $=64.8$ years) was used. PA (moderate-to-vigorous PA (MVPA), light-intensity PA (LIPA) and sedentary behaviour (SED)) during the awake time was measured with ActiGraph GT1M accelerometer at baseline, 6, 12, 18 and 24 months. Time in MVPA, LIPA and SED for each participant at each measurement point was converted to an $\mathrm{AC}$ (PA is from now referred to as the proportional time). Groups were created by splitting baseline values of the proportional time in MVPA into tertiles: low, middle, and high PA. The mean AC values in percentage for the three groups at each measurement point was calculated. Results: Figure 2 describes descriptive data on the $\mathrm{AC}$ in percentage for the low, middle, and high PA groups at baseline, 6, 12, 18 and 24 months. For the low PA group, MVPA were highest at 6 months and then decreased between 12 to 24 months. For the middle PA group MVPA were highest at 6 and 18 months, and lower at baseline, 12 and 24 months. For the high PA group, MVPA were highest at baseline, and then decreased during the twoyear period. Discussion: The low and middle PA groups seem to increase MVPA up to 6 months when the intervention was most intense. During the second year when the intervention was less intense the pattern of the activity composition varied. The high PA group decreased MVPA continuously during the whole two-year period. Regardless of initial PA, all individuals with type 2 diabetes and prediabetes might benefit from intense support to maintain beneficial PA levels over time.

Clinical and Mobility Measures to Discriminate Fallers From NonFallers in Parkinson's Disease

Rodrigo Vitorio ${ }^{1}$, Martina Mancini ${ }^{1}$, Patricia Carlson-Kuhta ${ }^{1}$, Fay Horak ${ }^{1}$, Vrutangkumar Shah ${ }^{1}$

${ }^{1}$ Oregon Health and Science University

Introduction: Although much is known about the multifactorial nature of falls in Parkinson's disease (PD), it remains unclear which measures best identify fallers. We aimed to identify both clinical and objective mobility measures that best discriminate fallers from non-fallers in PD. Methods: People with mild-to-moderate idiopathic PD were classified as fallers (at least one fall; $n=54)$ or non-fallers $(n=85)$ based on fall history in the previous six months. Clinical characteristics included demographic, motor and cognitive status and patient-reported outcomes. Mobility (balance and gait) characteristics were derived from 6 body-worn, inertial sensors while performing walking and standing tasks. Mobility outcomes included objective measures within 4 gait domains (upper body, lower body, turning and variability) and 3 postural sway domains (area, velocity and frequency). Model selection was done based on the 'best subsets selection strategy' on the training dataset $(80 \%)$ to find the best combinations of (up to four) measures in each scenario (i.e., clinical-only, mobility-only and combined clinical+mobility models) that best discriminate fallers from non-fallers. Logistic regression was applied on the test dataset (20\%) and the area under the curve (AUC) was calculated to evaluate the model performance. Results: The highest AUCs for the clinical-only, mobility-only and clinical+mobility models were $0.89,0.88$, and 0.94 , respectively. The most consistently selected measures in the top 10 ranked models were freezing of gait status $(8 x)$, the root mean square of anterior-posterior trunk acceleration while standing on a foam with eyes open $(5 x)$, gait double support duration $(4 \mathrm{x})$ and the postural instability and gait disorders score (4x; Figure 3 ). Discussion: These findings highlight the importance of considering multiple aspects of clinical as well as objective balance and gait characteristics for the classification of fallers and non-fallers in PD.

\section{Does a Cognitively Challenging Agility Bootcamp (ABC-C) Interven-} tion Improve Daily Physical Activity in Parkinson's Disease?

Hao Tan ${ }^{1}$, Kristan Dumas ${ }^{1}$, Graham Harker ${ }^{1}$, Matthew Welinski ${ }^{1}$, Patricia Carlson-Kuhta ${ }^{1}$, John Nutt ${ }^{1}$, Fay Horak ${ }^{1}$, Martina Mancini ${ }^{1}$

${ }^{1}$ Oregon Health and Science University

Introduction: Exercise interventions have been shown to improve mobility in people with Parkinson's disease (PD). We showed that an Agility Boot Camp intervention with Cognitive Challenge (ABC-C) improved mobility measured in the laboratory in people with PD. However, it is unknown if the ABC-C alters daily activity levels in people with $\mathrm{PD}$ or whether people with $\mathrm{PD}$ with marked gait impairments, such as freezing of gait (FoG) show a reduction in daily activities. This study aims to determine whether the ABC-C alters daily physical activity habits in people with $\mathrm{PD}$ and to understand how the presence of FoG impacts their response to the ABC-C. Methods: This is a cross-over, single-blind, randomized control trial. 42 subjects (18 with FoG) with PD wore an Actigraph (GT3X) on the waist throughout the study (12 weeks). Clinical measures of disease severity (MDS-UPDRS III and PIGD) were collected at baseline and after each intervention. The following measures of activity were averaged across 6-weeks each of exercise and education: step count, Kcal, sedentary time, moderate to vigorous physical activity (MVPA). T-tests were used to investigate differences after each intervention, and to detect activity differences between those with and without FoG. Results: Both people with and without FoG showed significantly higher mean daily MVPA and Kcal expenditure during the ABC-C compared to education $(\mathrm{p}<0.01)$. However, compared to those with FoG, subjects without FoG demonstrated a greater increase in Kcal expenditure and MVPA between the education and exercise interventions. A greater baseline disease severity, quantified by UPDRS III and PIGD scores, was correlated with lower Kcal expenditure, MVPA, and step count during the ABC-C. Discussion: The ABC-C increased daily physical activity in PD subjects, irrespective of FoG. However, results suggest that further improvements in daily life activities was achieved in individuals with milder disease at baseline.

\section{Exploring the Relationship Between Real-World Walking Speed and Motor Disease Severity in Parkinson's Disease: Insights From Cross- Sectional and Longitudinal Data}

Cameron Kirk ${ }^{1}$, Rana Rehman ${ }^{1}$, Brook Galna ${ }^{1}$, Lisa Alcock ${ }^{1}$, Saverio Ranciati $^{2}$, Luca Palmerini ${ }^{2}$, Judith Garcia-Aymerich ${ }^{3}$, Clint Hansen ${ }^{4}$, Eva Schaeffer $^{4}$, Lynn Rochester ${ }^{1}$, Silvia Del-Din ${ }^{1}$, Alison Yarnall ${ }^{1}$

${ }^{1}$ Newcastle University, ${ }^{2}$ University of Bologna, ${ }^{3}$ Barcelona Institute for Global Health (ISGlobal), ${ }^{4}$ Christian-Albrecht-University Kiel 
Introduction: Motor disease severity in Parkinson's disease (PD) is evaluated using the Movement Disorder Society Unified Parkinson's Disease Rating Scale part III (MDS-UPDRS III). This is considered the gold-standard, however, there are limitations associated with its use. Real-world walking speed (RWS), measured using wearable devices, has emerged as a potential tool to compliment the MDS-UPDRS III. The aim of this study was to explore the clinical value of RWS to assess motor severity by investigating the cross-sectional and longitudinal relationship with the MDS-UPDRS III. Methods: 213 PD participants were recruited from the Incidence of Cognitive Impairment with Longitudinal Evaluation - GAIT (ICICLE-GAIT) study. Participants underwent assessment of MDS-UPDRS III and RWS using a triaxial accelerometer (Axivity AX3) on the lower back at four time points. We employed linear regression to explore the cross-sectional relationship between RWS and MDS-UPDRS III and mixed effects models to investigate the longitudinal relationship between change in RWS and change in motor score over 54 months. Results: Cross-sectionally, a slower RWS was non-significantly associated with a higher UPDRS III $(\mathrm{R} 2=12 \%, \mathrm{P}=0.36)$. Longitudinally, a reduction of RWS over time was significantly related to an increase in the MDS-UPDRS III score $(\mathrm{R} 2=71 \%, \mathrm{P}=0.004)$. Discussion: Cross-sectionally, RWS was not significantly related to the MDS-UPDRS III. This lack of association could be explained by differences in the measurement conditions: while MDS-UPDRS III score was assessed when participants were optimally medicated (on-state), RWS captured the complexity of motor fluctuations due to 'on-' and 'off-states'. In contrast, longitudinal findings demonstrated that a deterioration in motor disease severity was significantly related to reduced RWS. RWS could therefore provide a complimentary tool to track the progression of the MDSUPDRS III.

\section{Monitoring Sleep-Related Parameters Using an Unobtrusive Bed Sensor in iSCI and Stroke Patients}

Maartje Hendriks ${ }^{1}$, Jaap van Lotringen ${ }^{1}$, Marije Vos- van der Hulst ${ }^{1}$, Noel Keijsers $^{1}$

${ }^{1}$ Sint Maartenskliniek

Introduction: Since adequate sleep is essential for optimal inpatient rehabilitation, there is an increased interest in sleep assessment. The aim of this study was to investigate the opportunities to measure sleeprelated parameters using an unobtrusive bed sensor in inpatient incomplete spinal cord injury (iSCI) and stroke patients. Methods: Patients with iSCI or stroke were monitored overnight for a 1-week period during their in-hospital rehabilitation using the Emfit QS bed sensor. Every morning, they filled out a questionnaire regarding their sleep of previous night. The following descriptive sleep-related parameters were reported: average hours of sleep, self-reported sleep score, nocturnal heart rate, respiratory rate, movement activity, and difference between self-reported and recorded bed exits per night. Differences between the iSCI and stroke group were calculated. Results: In total, sleep-related parameters of 20 participants (11 iSCI, 9 stroke) were measured. Sleeprelated parameters can be found in Figure 4. Patients with a stroke had a significantly higher self-reported sleep score compared to iSCI patients. Patients with an iSCI had significantly higher nocturnal heart rate and movement activity compared to stroke patients. Furthermore, there was a difference between self-reported and recorded bed exits per night in $26 \%$ and $38 \%$ of the nights for iSCI and stroke patients, respectively. Discussion: This is a first orientation in possibilities of sleep-related parameters which can be measured using an unobtrusive bed sensor in the clinical rehabilitation setting. Moreover, differences between patients with stroke and iSCI were found.

\section{Objective Monitoring of Functional Recovery After Total Knee and Hip Arthroplasty Using Sensor-Derived Gait Measures}

Ramon Boekesteijn ${ }^{1}$, José Smolders ${ }^{1}$, Vincent Busch ${ }^{1}$, Noël Keijsers ${ }^{1}$, Sander Geurts ${ }^{2}$, Katrijn Smulders ${ }^{1}$

${ }^{1}$ Sint Maartenskliniek, ${ }^{2}$ Radboud University Medical Centre

Introduction: Restoration of physical functioning is a major aim of total joint arthroplasty, which is typically evaluated by subjective patientreported outcome measures (PROMs). In this study, we aimed to investigate objective recovery of gait - including turning, upper body motion, and gait automaticity - after total knee (TKA) and hip arthroplasty (THA), and to compare post-operative functioning with healthy controls (HC). Methods: Gait was assessed in individuals pre-operatively, and at 2 and 15 months after TKA $(n=24)$ and THA $(n=24)$, and compared with HC $(n=27)$ of similar age. Data were collected using inertial measurement units (IMUs) on the feet, lower back, and sternum. Participants walked for 2 minutes back and forth over a $6 \mathrm{~m}$ walkway with $180^{\circ}$ turns, with and without a secondary cognitive task. PROMs were obtained using the knee osteoarthritis outcome score (KOOS) and hip disability and osteoarthritis outcome score (HOOS). Results: Gait speed improved 15 months after TKA by $0.18 \mathrm{~m} / \mathrm{s}$ and after THA by $0.17 \mathrm{~m} / \mathrm{s}$, reaching HC values. Three (of 24 TKA) and five (of 24 THA) participants did not reach a clinically meaningful improvement in gait speed $(\geq 0.10 \mathrm{~m} / \mathrm{s})$. After TKA and THA, turning and upper body motion parameters improved toward $\mathrm{HC}$ values. Dual-task costs were not different from those of healthy controls, either before or after surgery. Recovery patterns of gait parameters were more gradual than the HOOS and KOOS recovery profiles (see Figure 5). Discussion: Gait assessments with IMUs showed that next to spatiotemporal parameters, turning and upper body motion recovered to a similar level as HC. Yet, a substantial number of individuals after TKA and THA did not reach a clinically meaningful improvement in gait speed, irrespective of good PROM scores. In contrast to large and early improvements in PROMs, objective measures showed more gradual responses, demonstrating added value for clinical evaluations after TKA and THA.

\section{Parkinson's Disease and Visual Cueing: Effects on Gait Initiation and Walking}

Yuri Russo $^{1}$, Giuseppe Vannozzi ${ }^{1}$, Martina Mancini ${ }^{2}$

${ }^{1}$ University of Rome Foro Italico, ${ }^{2}$ Oregon Health and Science University

Introduction: People with Parkinson's Disease (PD) show impaired gait initiation (GI) - e.g., reduced Anticipatory Postural Adjustments (APAs) - compared to controls; in addition, such impairments are likely to be more severe for those suffering from freezing of gait (FOG). Visual cueing devices have been used to improve PD gait parameters, although their effect on GI is understudied. In the present study we investigated the impact of personalized visual cues, as transverse taped lines on the floor, on GI and gait parameters in PD with/without FOG (FOG+/FOG-). Methods: Controls (HC), FOG- and FOG+ were enrolled in the study. Participants were asked to start walking forward after five seconds of static posture; all volunteers performed the task both with and without cues, which were placed at $120 \%$ of their natural step length. APAs parameters (i.e. MedioLateral APAs size and duration) together with gait spatiotemporal parameters (i.e. step length and gait velocity) were measured by 3 inertial measurement units (Opals by APDM), on both feet and waist. Results: The groups did not differ for demographics or clinical characteristics, except for FOG+ being more severe compared to FOG- (MDS-UPDRS). FOG+ had smaller ML APAs compared to HC and FOG- (Figure 6A). No differences were found between HC and FOG-. Visual cueing reduced the size of APAs and lengthened APAs duration, yet significantly increased both stride length and gait speed (Figure 6B). No significant 
interactions were found. Discussion: Prior to walking, FOG+ produced smaller APAs compared to HC and FOG-, whereas no difference was found between the latter groups. APAs of the three groups were affected by visual cues in a similar way (i.e increased duration and reduced size). Contrarily, a marked increase of gait spatiotemporal parameters was observed when cueing was applied. Therefore, it is possible that postural preparation may not be as relevant when participants are presented with a signaled walking path.

\section{Saccade and Fixation Eye Movements During Walking in Mild Traumatic Brain Injury and Healthy Controls}

Ellen Lirani-Silva ${ }^{1}$, Samuel Stuart ${ }^{1}$, Lucy Parrington ${ }^{2}$, Kody Campbell ${ }^{2}$, Laurie King ${ }^{2}$

${ }^{1}$ Northumbria University, ${ }^{2}$ Oregon Health and Science University

Introduction: Clinical and laboratory assessment of people with mild traumatic brain injury (mTBI) indicate impairments in eye movements. These tests are typically done in a static, seated position. Recently, the use of mobile eye-tracking systems has been proposed to quantify subtle deficits in eye movements and visual sampling during different tasks. However, the impact of mTBI on eye movements during functional tasks such as walking remains unknown. The aim of this study was to evaluate differences in eyetracking measures during walking between healthy controls (HC) and mTBI individuals. Methods: Thirty-seven HC participants and 67 mTBI were instructed to walk back and forth over 10-meters, at a self-selected speed. A single 1-minute trial was performed. Eye-tracking measures were recorded using a mobile eye-tracking system (head-mounted infra-red Tobbii Pro Glasses 2, Tobii Technology Inc., VA, USA). Eye-tracking measures included saccadic (frequency, mean and peak velocity, duration and distance) and fixation measurements (frequency and duration). Gait was assessed using six inertial sensors (both feet, sternum, right wrist, lumbar vertebrae and the forehead) and gait velocity was selected as the primary outcome. Independent t-tests and Mann-Whitney $U$ tests were used to compare the groups. Results: Individuals with mTBI showed significantly reduced saccadic frequency $(\mathrm{p}=0.003)$, duration $(\mathrm{p}=0.006)$ and peak velocity $(\mathrm{p}=0.007$ ) compared to the HC group. No significant differences between groups were observed for the saccade mean velocity and distance, fixation measures and gait. Discussion: Findings suggest impaired saccadic eye movement, but not fixations, during walking in mTBI individuals. These findings have implications in real-world function including return to sport for athletes and return to duty for military service members. Future research should investigate whether or not saccade outcomes are influenced by the time after the trauma and rehabilitation.

\section{Sensor-Derived Physical Activity in People With Parkinsons Disease During the First Wave of Covid-19 Pandemic - A Cross-Sectional Study From Sweden}

Maria Hagstromer ${ }^{1}$, Breiffni Leavy ${ }^{1}$, David Moulaee Conradsson ${ }^{1}$, Erika Franzén ${ }^{1}$

${ }^{1}$ Karolinska Institutet

Introduction: People with Parkinson's disease (PD) are at risk of physical inactivity and may be especially vulnerable to negative health outcomes during the COVID-19 pandemic due to social distancing recommendations. The objective of this study was to investigate sensor-derived physical activity and its associated factors in people with mild-moderate PD during the first wave of the COVID-19 epidemic. Methods: Participants ( $\geq 60$ years) were recruited from an existing cohort and through advertisement. Data was collected during the first wave of the COVID-19 pandemic. Physical activity was measured over 7-days using the Actigraph GT3x accelerometer. Outcome variables was steps per day and time spent in intensity levels using cut-points for older adults. Data collection also incorporated perceived health status, exercise habits, and provision of rehabilitation during the pandemic. Multiple linear and logistic regression analyses were used to identify factors associated with physical activity (steps per day). Results: In total 89 participants with mean age $71.0 \pm 6.0$ years, disease duration $6.0 \pm 4.3$ years were included. Study participants took on average $5876 \pm 3180$ steps per day, spent 598 minutes sedentary, 197 minutes in low intensity physical activity and 47 minutes in moderate to vigorous physical activity. A majority $(67 \%)$ reported a pandemicrelated reduction in exercise habits, almost half of all participants reported cancelled rehabilitation and $40 \%$ took less than 5000 steps per day. In the multivariate analysis, female gender, being $\geq 70$ years, and greater reported mobility problems were associated with being less physically active. Discussion: Despite a pandemic-related reduction in reported exercise habits and rehabilitation, participants in this Swedish sample were relatively physically active. However, women, $\geq 70$ years, with mobility problems, were at greater risk of low levels of physical activity.

\section{The Effects of a Multidisciplinary Exercise Intervention in Patients With Parkinson's disease: In-Lab Testing and Community Ambula- tion Respond Differently}

Moriya Cohen ${ }^{1}$, Natalie Ganz ${ }^{2}$, Talia Herman ${ }^{2}$, Yitchak Green ${ }^{3}$, Inbal Badichi $^{3}$, Tanya Gurevich ${ }^{2}$, Jeffrey Hausdorff ${ }^{2}$

${ }^{1}$ Tel-Aviv University, ${ }^{2}$ Tel-Aviv Sourasky Medical Center, ${ }^{3}$ Ezra Lemarpeh

Introduction: Multidisciplinary exercise interventions can improve gait and balance in patients with Parkinson's disease (PD). However, it is not yet known if they also positively impact the quality or quantity of dailyliving walking. Methods: 46 PD patients (age: $70.05 \pm 7.71$; gender: $31.3 \%$ women; disease duration: $8.85 \pm 6.27 \mathrm{yrs}$ ) were evaluated before and after participating in 8-weeks of physical, occupational, and hydro-therapy, boxing, and dance ( 3 days/week; 5 hrs/day). Gait and balance were measured in the clinic. A 3D accelerometer was placed on the subjects' lower back to measure daily-living walking for 1-week before and after the intervention. Results: After the intervention, clinical measures of gait and balance improved, e.g.: MiniBest balance test delta: $1.82 \pm 3.30$ points, $\mathrm{p}=0.001$; Timed Up and Go delta: $-1.78 \pm 6.15 \mathrm{sec} ; \mathrm{p}=0.001$, and usual-walking gait speed delta $19 \pm 16 \mathrm{~cm} / \mathrm{s} ; \mathrm{p}<0.001$. In contrast, the number of steps and gait quality did not change $(\mathrm{p}>0.5)$. In exploratory analyses, subjects were categorized as responders (Rs) and non-responders (NRs) based on their change in everyday walking gait speed. Rs increased their daily-living gait speed (delta: $10 \pm 14 \mathrm{~cm} / \mathrm{s} ; \mathrm{p}<0.001$ ), whereas NRs did not. Rs $(\mathrm{n}=21)$ improved daily-living gait quality measures (e.g. stride regularity, step length, stride time variability, and sample entropy). At baseline, the disease severity was lower in Rs, compared to the NRs (MDS-UPDRSIII: Rs: $25.33 \pm 11.47$, NRs: $34.38 \pm 14.27$; $\mathrm{p}=0.02$ ). Discussion: These results demonstrate that improvements in PD in the clinic do not necessarily carry over to improvements in dailyliving gait; daily-living gait does not mirror gait measured in the clinic. The findings also suggest that in select patients with PD, it is possible to ameliorate daily-living walking quality, potentially reducing the risk of falls and other adverse outcomes associated with impaired mobility.

Using a Body-Fixed Accelerometer to Characterize Impairments in Daily-Living Physical Activity and Gait in Patients With Probable Idiopathic Normal Pressure Hydrocephalus

Michal Elias ${ }^{1}$, Inbar Hillel ${ }^{2}$, Eran Gazit ${ }^{2}$, Marina Brozgol$^{2}$, Ira Galperin ${ }^{2}$, Jeffrey Hausdorff ${ }^{2}$, Elissa Ash $^{2}$

${ }^{1}$ Tel Aviv University, ${ }^{2}$ Tel-Aviv Sourasky Medical Center 
Introduction: Idiopathic Normal Pressure Hydrocephalus (iNPH) is characterized by ventriculomegaly, gait disturbances, cognitive decline, and urinary incontinence. It is treated by surgical cerebrospinal fluid (CSF) diversion. The CSF tap test (TT) is used to select patients who may benefit from shunting, with sub-optimal sensitivity. Quantitative gait assessments may increase TT sensitivity, but have been limited to laboratory/clinical settings. We explored the feasibility of extracting daily-living mobility features using wearables to characterize and quantify gait impairments in iNPH, and to detect gait improvements in response to TT. Methods: Exploratory analyses were performed in 14 patients with probable iNPH and 20 age-matched healthy controls (HC). Subjects wore a lower back accelerometer continuously to evaluate the amount and quality of their activity. Pre-Post TT evaluations were performed in $12 \mathrm{iNPH}$ participants during usual and dual-task (DT) walking in the laboratory and during daily-life ambulation. Participants were classified as responders $(n=7)$ and non-responders $(n=5)$ based on clinical evaluation. Results: Probable iNPH patients were less active $(\mathrm{p}=0.014)$, took fewer steps $(\mathrm{p}=0.008)$, walked more slowly $(\mathrm{p}=0.007)$, with greater asymmetry and variability ( $p<0.001)$ during community ambulation. In-lab analyses revealed that TT responders walked faster $(\mathrm{p}=0.027)$, with larger step length and lower stride time $(\mathrm{p}=0.028)$ during usual walking. Improvements in asymmetry and variability of gait $(\mathrm{p}=0.018)$ were observed in DT walking. Dailyliving gait did not change post TT. Discussion: These findings show that during community ambulation, probable iNPH patients have marked gait alterations in multiple mobility domains, compared to their healthy peers. TT responders showed improvements in the laboratory which were not observed in the real-life environment, in this small sample. These preliminary findings demonstrate the feasibility of the assessments. Larger-scale studies may aid in the iNPH evaluation.

Using an Unobtrusive Bed Sensor to Monitor Spasticity During the Night: Preliminary Results

Maartje Hendriks ${ }^{1}$, Merel Rougoor ${ }^{1}$, Marije Vos- van der Hulst ${ }^{1}$, Noel Keijsers $^{1}$

\section{${ }^{1}$ Sint Maartenskliniek}

Introduction: Spasticity is a common symptom in various neurological conditions and can limit daily life activities as well as disturb sleep. As sleep is an important part of the rehabilitation, objective unobtrusive monitoring of nocturnal spasticity is important, yet not available. Therefore, the aim of this study was to determine if spasticity in the legs can be measured during the night by using contactless unobstructive bed sensors in neurological patients with nocturnal spasticity. Methods: Neurological patients with or without spasticity were monitored overnight for 3 consecutive nights during their in-hospital rehabilitation using two highly sensitive pressure sensors (Emfit QS bed sensors) placed at thorax and leg height. Participants were divided in three groups based on the presence of spasticity and their ability to perform voluntary movements: I) spasticity and voluntary movement (S\&VM), II) spasticity and no voluntary movement (S\&NVM), III) no spasticity and voluntary movement (NS\&VM). Primary outcome was the mean power for frequencies between $2-8 \mathrm{~Hz}$. Results: Up until now, 13 neurological patients were measured distributed along three groups (S\&VM: 4; S\&NVM: 4; NS\&VM: 5). Mean power measured with the leg bed sensor was $226.8 \pm 83,60.0 \pm 46$, and $93.2 \pm 58$ for respectively S\&VM, S\&NVM, and NS\&VM. Kruskal-wallis revealed a significant difference between groups $(\mathrm{p}=.035)$. Mean power measured with the thorax bed sensor was $362.4 \pm 169,231.8 \pm 133$, and $319.0 \pm 192$ for respectively S\&VM, S\&NVM, and NS\&VM, which was not significantly different between groups $(p=.60)$. Discussion: A bed sensor at leg height is able to distinguish groups based on high frequency movements. However, no clear association between nocturnal spasticity and high frequency movements. Group expansion and comparison with inertial measurement units around the ankles are necessary, to investigate the potential of unobtrusive bed sensors to measure nocturnal spasticity.

\section{Applications: Specific Populations}

Comparison of Physical Function and Incidental Physical Activity Between Two Categorical Blood Glucose Levels in a Target Range Amongst People With Type 2 Diabetes

Tal Yahalom-Peri ${ }^{1}$, Einat Kodesh ${ }^{2}$, Yamit Basson-Shleymovich ${ }^{1}$, Michal Azmon $^{1}$, Veronica Bogina ${ }^{1}$, Tsvi Kuflik ${ }^{1}$, Tali Cukierman-Yaffe ${ }^{1}$

${ }^{1}$ Sheba Medical Center, Israel, ${ }^{2}$ University of Haifa, Israel

Introduction: Physical activity is reported to be important for blood glucose management and overall health. Individuals with diabetes are encouraged to engage in exercise and to increase their total daily incidental physical activity (IPA). Continuous glucose monitoring (CGM) identifies an individual's current glycemic status and provides valuable information about the amount of time spent in the recommended glucose target range (70$180 \mathrm{mg} / \mathrm{dL}$ ), defined as Time in Range\% (TIR). In this study we aimed to compare the physical function and IPA between individuals with a lower $(\leq 70 \%)$ and higher $(>70 \%)$ TIR. Methods: Twenty participants were recruited at "The Center for Successful Aging with Diabetes", Sheba Medical Center. They underwent elaborate physical-functional tests including the 6-minute walk test (6MW) to asses aerobic capacity and the Sitto-Stand test (STS) to asses leg strength. They were connected to a CGM system (I Pro2 carelink, Medtronic) and wore an activity device (ActiGraph GT9X) on their waist for a week. Number of steps, sedentary and light to vigorous activity time were recorded during 5 consecutive days. Individuals were divided into 2 groups according to the TIR\% obtained from the CGM system ( $>70 \%$ and $\leq 70 \%$ ), all outcome measurements were compared between the groups. Results: The $>70 \%$ TIR group demonstrated significant $(\mathrm{p}=0.04)$ higher performance on the STS test (Mean Diff. 5.95 seconds) and trend towards statistical significance $(\mathrm{p}=0.07)$ on the $6 \mathrm{MW}$ (Mean Diff. 113.29 meter). No significant differences were found between the groups in the number of steps, and time in sedentary, light to vigorous activity. Discussion: In this study, participants with higher \%TIR performed better in functional tests, however their incidental daily physical activity was no different from the group with lower \% TIR. We also applied machine learning techniques for identifying recurring patterns between the participants. Larger studies are needed to further validate these findings.

Objective Measurement of 24-Hour Movement Behaviors in Preschool Children Using Wrist-Worn and Thigh-Worn Accelerometers Marieke De Craemer ${ }^{1}$, Marga Decraene ${ }^{2}$, Iris Willems ${ }^{2}$, Vera Verbestel ${ }^{2}$ ${ }^{1}$ Ghent University \& Research Foundation Flanders, ${ }^{2}$ Ghent University

Introduction: In recent years, more attention has been paid towards the study of 24-hour movement behaviors (including physical activity (PA), sedentary behavior (SB) and sleep) in preschoolers instead of studying these behaviors in isolation. This study aimed to evaluate the feasibility of using wrist- vs. thigh-worn accelerometers and to report accelerometerderived metrics of 24-hour movement behaviors in preschoolers. Methods: A convenience sample of 17 preschoolers ( $47.1 \%$ boys, 3.75 years) and one of their parents were recruited for this study. Preschoolers had to wear the ActivPAL accelerometer (attached to the upper thigh) and Axivity accelerometer (using a wrist band) simultaneously for 7 consecutive days and 24 hours a day. Parents completed an acceptability survey. Results: All 17 preschoolers $(100.0 \%)$ had 7 days of valid wrist-worn data, while only 12 preschoolers $(70.6 \%)$ had 7 days of valid thigh-worn data $(\mathrm{p}=0.03)$. When looking at the acceptability, 58.8\% of parents indicated that it was 
easy for their child to wear the Axivity for 7 consecutive days, and $64.7 \%$ of parents indicated so for the ActivPAL $(p=0.39)$. However, some parents stated that the wrist-band of the Axivity accelerometer was big which might have affected data collection. Significant differences were found for the measurement of total volume of PA and SB across 24 hours. Total PA was $474.23 \mathrm{~min} /$ day $( \pm 76.14)$ with the ActivPAL compared to $345.83 \mathrm{~min} /$ day $( \pm 54.50)$ with the Axivity $(\mathrm{p}<0.001)$. The volume of SB was $314.17 \mathrm{~min} /$ day ( \pm 66.69 ) with the ActivPAL compared to $445.75 \mathrm{~min} /$ day $( \pm 56.60)$ with the Axivity $(\mathrm{p}<0.001)$. No significant difference was found for sleep $(\mathrm{p}=0.98$; ActivPAL: $651.58 \mathrm{~min} /$ day \pm 34.29 ; Axivity: $651.25 \mathrm{~min} /$ day \pm 51.64 ). Discussion: Overall, parents perceived both devices to be feasible to use for preschoolers but future studies are required to validate both devices for the measurement of preschoolers' 24-hour movement behaviors.

\section{Relationship Between Gross Motor Skills and Physical Activity in Toddlers}

Sara King-Dowling ${ }^{1}$, Natascja Di Cristofaro ${ }^{2}$, Joyce Obeid ${ }^{1}$

${ }^{1}$ Children's Hospital of Philadelphia, ${ }^{2}$ McMaster University

Introduction: Motor skill competence is an important predictor of physical activity (PA) in older children and adolescents, however few studies have examined this relationship in children under the age of 3 . The purpose of the current study was to examine if gross motor skills are associated with device-measured PA in toddlers. Methods: Toddlers $(n=109)$ age $12-35$ months $($ Mage $=21.3 \mathrm{mos}, \mathrm{SD}=7.1 ; 51.4 \%$ girls) were recruited as part of the investigating the validity and reliability of accelerometer-based measures of PhysicaL Activity and sedentarY time in toddlers (iPLAY) study. Gross motor skills (including subdomains for stationary, locomotor, and object manipulation) were assessed using the Peabody Developmental Motor Scales 2nd Edition (PDMS). PA was measured with an accelerometer (ActiGraph wGT3X) worn around the waist for 7 days. Average daily vector magnitude counts per min (cpm) were calculated for children who wore the accelerometer for at least 6 hours on at least 4 days. Linear regressions controlling for age and sex were conducted to examine if overall gross motor skills, or any subdomains, were associated with PA. Results: Ninety-seven (89\%) toddlers had valid PA and motor assessments. Boys were more active than girls $(\mathrm{Mcpm}=1231.2$ vs. $1134.8, \mathrm{t}=2.1, \mathrm{p}=.04)$ and age was positively associated with PA $(\mathrm{r}=.38, \mathrm{p}<.01)$. Neither overall gross motor skills $(\mathrm{B}=0.51, \mathrm{p}=.47)$ nor any of the subdomains (Stationary $\mathrm{B}=-1.15, \mathrm{p}=.40$; Locomotor $\mathrm{B}=0.71, \mathrm{p}=.49$; Object Control $\mathrm{B}=0.92, \mathrm{p}=.38$ ) were associated with PA in toddlers. Discussion: This work confirms previous reports and supports motor developmental frameworks where the association between motor competence and PA emerges over time as children age. Given the unique movement patterns of toddlers, further research should examine these relationships using novel accelerometer metrics. Although not associated in this age group, promoting gross motor skill development may have implications for later PA.

\section{Applications: COVID-19}

Physical Activity Behaviour and Screen Time in Dutch Children During the COVID-19 Pandemic: Pre, During and Post School Closures

Gabrielle ten velde ${ }^{1}$, Judith Lubrecht ${ }^{1}$, Lisanne Arayess ${ }^{1}$, Christiana van Loo $^{1}$, Marijn Hesselink ${ }^{1}$, Dorien Reijnders ${ }^{1}$, Anita Vreugdenhil ${ }^{1}$

\section{${ }^{1}$ Maastricht University}

Introduction: Measures during the COVID-19 pandemic, including the closure of schools and sports facilities, may have lasting impact on the physical activity (PA) of children that persists for a long time. Objective:
To investigate the effect of COVID-19 measures on screen time and PA in Dutch children pre, during and post school closures. Methods: PA and screen-time data were collected from two different cohorts. In cohort A ( $\mathrm{n}=102,10.5 \pm 3.6 \mathrm{y}, 42.4 \%$ boys) data during the lockdown was collected using a questionnaire. Cohort B was derived from an on-going study ( $\mathrm{n}=131,10.2 \pm 0.9 \mathrm{y}, 43.5 \%$ boys). Data on PA and screen time were collected using a questionnaire and accelerometry 1 year before (may 2019) and after the controlled lockdown i.e. school closure (June 2020). Results: In cohort A, $62 \%$ reported less total PA. Self-reported screen time on week days increased $34 \pm 105 \mathrm{~min} /$ day during the lockdown. In cohort $\mathrm{B}$, sedentary time as measured by accelerometry, increased by $45 \pm 67 \mathrm{~min} /$ day and only $20 \%$ reached PA levels of $60 \mathrm{~min}$ /day compared to $64 \%$ in May 2019. Self-reported screen time increased by $59 \pm 112 \mathrm{~min} /$ day and $62 \pm 130 \mathrm{~min} /$ day during week and weekend days, respectively. Discussion: Children were less physically active and screen time was higher during and after the school closures due to the COVID-19 lockdown. This is alarming as an active lifestyle in children is crucial in preventing chronic diseases such as obesity.

\section{The Impact of the COVID-19 Pandemic on Symptoms, Activity and Quality of Life in People Living With Dystonia}

\author{
Silmara Gusso ${ }^{1}$, Rebecca Meiring ${ }^{1}$, Lynley Bradnam ${ }^{1}$ \\ ${ }^{1}$ University of Auckland
}

Introduction: People with dystonia frequently visit hospital for the management of their condition with BOTOX, however during the COVID-19 pandemic, clinics were closed, and many people were unable to access services. This study aimed to determine how COVID-19 restrictions affected people with dystonia and how they managed their symptoms during lockdown. Methods: An online survey was distributed internationally through dystonia support groups and social media in June 2020. Collected data included demographics, closed and open-ended questions regarding access to care and their impact on dystonia. The dystonia nonmotor symptom scale and the SF-36 were also used to measure the impact of dystonia on quality of life (HRQoL). Results: There were 72 responses $(\mathrm{n}=60$ female, mean 56 years, cervical dystonia: $27 \%$ ). Twenty five percent of respondents had their appointment delayed due to COVID restrictions and $66 \%$ had another one scheduled. Most (70\%) respondents were less active than usual due to fatigue, dystonic symptoms and disturbed sleep. Those that continued to exercise felt motivated, and reporting using exercise to manage dystonia. About $54 \%$ reported their pain was made worse than usual while $40 \%$ reported it was same. The nonmotor symptoms made worse than usual were fatigue, pain, sleep, walking/ balance, and mood. HRQoL results revealed low scores for role limitation in physical activities (median (IQR): 25 (0-75), physical (36 (29-45) and mental health (39 (19-50), fatigue (40 (30-50), and pain (45 (35-58). Around $30 \%$ of respondents reported thinking about their dystonia more than usual. Commonly used self-management strategies were walking $(63 \%)$, general exercise $(41 \%)$, mindfulness $(37 \%)$, and meditation $(34 \%)$. Discussion: COVID-19 restrictions negatively impacted people with dystonia, especially non-motor symptoms of pain, fatigue and sleep. Physical activity and aspects of HRQoL were reduced. Self-management techniques may assist coping with increased symptoms. (Figure 7)

\section{Applications: Public Health}

Activity Bout Accumulation Patterns in Two Clinical Samples Using the Fitbit Charge 2 Activity Monitor

Kristina Hasanaj $^{1}$, Krista Leonard ${ }^{1}$, Sarah Kozey-Keadle ${ }^{2}$, Megan Petrov ${ }^{1}$, Matthew Buman ${ }^{1}$

${ }^{1}$ Arizona State University, ${ }^{2}$ California Polytechnic State University 
Introduction: US and global physical activity guidelines now recommend moderate-vigorous physical activity (MVPA) can be accrued in any bout length for health benefits. Researchers often rely on research-grade devices (e.g. activPAL) and/or self-report to examine activity bout accumulation patterns. There are limited reports of bout accumulation patterns in consumergrade activity monitors (e.g. Fitbit), especially in clinical populations. This study aimed to describe activity bout accumulation patterns in Fitbit-measured activity (total activity, MVPA) in two clinical samples. Methods: Data were pooled from two sedentary clinical samples, one with obstructive sleep apnea and one with prediabetes; both had overweight/obesity. Participants wore a Fitbit Charge 2 activity monitor for 60-270 days. Minute-level activity and associated metabolic equivalents (METs) were directly accessed through the Fitbit application programming interface. Total activity was classified as METs $\geq 1.5$ and MVPA as METs $\geq 4$.0. SAS arrays summarized continuous activity bouts/day of $2,3,4,5,6,7,8,9$, and 10min. Results: Participants from the prediabetes sample $(n=60,73 \%$ male, $34.6 \pm 6.9$ BMI, $56.1 \pm 10.9 \mathrm{yr})$ and the obstructive sleep apnea sample $(\mathrm{n}=101,58 \%$ male, $33.0 \pm 6.5 \mathrm{BMI}$, $57.9 \pm 12.5 \mathrm{yr}$ ) had $69 \% \pm 24 \%$ days of valid wear ( $\geq 10 \mathrm{hr}$ waking weartime). The median number and length of bouts, and min of total activity and MVPA are in Figure 8. Discussion: According to this rich time-series Fitbit data in two clinical samples, total activity and MVPA were accrued in short bouts of activity, with substantial heterogeneity across individuals, and levels that substantially exceed current guidelines. A minute-level threshold for activity bout duration was not apparent. This patterning of physical activity confirms strategies to encourage even small, interrupted activity bouts may increase total activity, which is likely to accrue similar health benefits as fewer but more sustained bouts of activity.

\section{Alternatives for Measuring Sitting Accumulation in Workplace Surveys}

Bronwyn Clark $^{1}$, Samantha Stephens ${ }^{1}$, Ana Goode ${ }^{1}$, Genevieve Healy ${ }^{1}$, Elisabeth Winkler ${ }^{1}$

${ }^{1}$ The University of Queensland

Introduction: Reducing and breaking up sitting time are key targets for behaviour change in desk workers; however, the few available self-report measures of sitting time accumulation have only modest measurement properties. This study assessed the measurement qualities of a range of questions that may be suitable for measuring sitting time accumulation in the workplace. Methods: Workers from three office environments $(n=51$, mean age $=43$ years, $83 \%$ female) completed an online questionnaire immediately before and after wearing the activPAL3TM for 7 days (24-h protocol, diary-reported work hours). They reported typical behaviour at work in the past week: percentage of sitting in $\geq 30$ minute bouts (\% long sitting); number of interruptions per hour sitting ( $\mathrm{n}$ interruptions); number of strategies to breakup sitting used (n strategies); and, total times per day strategies were used (nx strategies). Minimal detectable change (MDC), testretest reliability (first versus second response) and criterion-validity (versus monitor) were assessed, testing agreement whenever possible. Results: Testretest intra-class correlations were 0.66 for $\%$ long sitting, 0.71 for $\mathrm{nx}$ strategies, 0.73 for $\mathrm{n}$ strategies (all $\mathrm{p}<0.05$ ), and 0.05 for $\mathrm{n}$ interruptions $(\mathrm{p} \geq 0.05)$. Consequently, MDC was higher for $\mathrm{n}$ interruptions $(186 \%)$ than n strategies (34\%), nx strategies (61\%) and \% long sitting (50\%). Spearman's correlations with criteria were 0.34 for $n x$ strategies, 0.42 for $\%$ long sitting, and 0.48 for $\mathrm{n}$ interruptions (all $\mathrm{p}<0.05)$ and $0.21(\mathrm{p} \geq 0.05)$ for $\mathrm{n}$ strategies. Mean difference $(95 \%$ agreement limits) was $-6(-58,47)$ for $\%$ long sitting. Discussion: Reliability and validity were better for $\%$ long sitting and $\mathrm{nx}$ strategies than for other accumulation measures reported previously and tested here, approaching levels of commonly used measures of total workplace sitting time. These questions may be useful additions for workplace studies requiring self-report options.
Association Between Parental and Child Objectively Measured Physical Activity: Results From a Brazilian Populational Birth Cohort Study

Luiza Ricardo $^{1}$, Ricardo Oliveira ${ }^{1}$, Cauane Blumemberg ${ }^{1}$, Debora Tornquist $^{1}$, Luciana Tornquist ${ }^{1}$, Inacio Crochemore-Silva ${ }^{1}$

${ }^{1}$ Federal University of Pelotas

Introduction: The present study aims to verify the association between parental and child physical activity (PA), using accelerometry, in a Brazilian populational cohort - the 2015 Pelotas birth cohort. Methods: The present study includes participants from the 2015 Pelotas birth cohort, which includes all live-born infants from the urban region of Pelotas in 2015. Paternal, maternal and child physical activity was measured for 7 consecutive days using accelerometers (ActiGraph, model wGT3X-BT, ActiGraph, USA), in the 12-, 24- and 48- months followups, respectively. Data processing was performed with R-package GGIR. All data was expressed in its raw form $(\mathrm{mg})$, with 5-s epochs, in a continuous form for children. Parental moderate-to-vigorous PA was calculated using 10 minutes bouts, and divides in tertiles. Crude and adjusted (income, maternal/paternal education, and maternal age) analyses were performed using linear regressions. Stata 16 software was used for all statistical analyses. Results: A total of 2955 children had valid accelerometer data, and 1326 had both parents with valid accelerometry. Overall child PA mean was $48.1 \mathrm{mg}(\mathrm{sd}=11.2)$, being higher among boys (boys: $50 \mathrm{mg}$, sd $=11.9$; girls: $46 \mathrm{mg}$, sd $=9.9$; p-value $<0.001$ ). Mean maternal MVPA was 9.1 minutes $(\mathrm{sd}=13.1)$, while paternal MVPA mean was $20.4(\mathrm{sd}=31.1)$. Child PA was positively associated with maternal MVPA at 24 months (Crude $\mathrm{p}=0.004$; Adjusted $\mathrm{p}=0.021$ ) and paternal MVPA at 12 months (Crude $\mathrm{p}=0.01$; Adjusted $\mathrm{p}=0.012$ ). Also, a child with both parents in the 3rd tertile of MVPA presented higher overall PA (Crude $\mathrm{p}<0.001$; Adjusted $\mathrm{p}=0.01$ ). Discussion: Our study showed a positive effect of maternal and paternal PA on child PA, being slightly more pronounced for mothers, and even stronger when both parents are active. Parental PA appears to be a consistent determinant of child PA, which could represent a behavioral influence, in which active parents tend to incentive the movement and active play (Figure 9).

\section{Associations Between Physical Activity and Cardiovascular Risk Factors Among Dutch Children}

Gabrielle ten velde ${ }^{1}$, Guy Plasqui ${ }^{1}$, Maartje Willeboordse ${ }^{1}$, Bjorn Winkens $^{1}$, Anita Vreugdenhil ${ }^{1}$

${ }^{1}$ Maastricht University

Introduction: Physical activity (PA) plays an important role in the prevention of cardiovascular diseases, especially for children. Previous studies which investigated PA and sedentary time (ST) used different measures which create inconsistency of results. The current study used recommended standardized PA measures and provides an overview of PA and ST among Dutch primary school children and their associations with cardiovascular risk factors. Methods: 503 children (55\% girls, $10 \pm 1 \mathrm{y}$ of age) were included. PA (total PA, light PA and moderate to vigorous PA (MVPA)) and ST were measured with the GT3X Actigraph accelerometer. Cardiovascular risk factors included BMI z - score, waist circumference, blood pressure and estimated cardiorespiratory fitness (CRF). Results: Children spent $57 \pm 20 \mathrm{~min} /$ day $(8 \%)$ on MVPA and $41.6 \%$ reached the MVPA guideline of $60 \mathrm{~min} /$ day. Total PA and MVPA were negatively associated with BMI z-score $(\mathrm{B}=-0.452, \mathrm{p}=0.011)$ and waist circumference $\mathrm{B}=-3.553, \mathrm{p}=0.011$ ) and positively associated with $\mathrm{CRF}$ $(B=2.527, p \leq 0.001)$. ST was associated with BMI z-score $(B=0.108$, $p=0.048)$ and waist circumference $(B=0.920, p=0.033)$. No associations were found between PA and blood pressure. Discussion: The findings 
emphasize the importance of promoting MVPA in children, but also highlight the potential benefits of reducing ST to improve cardiovascular risk factors.

\section{Comparison of Activity Monitors and Bout-Level Analysis Methods in} the Study of Daily-Life Walking Pattern in Older Adults

Adrien Chanteau ${ }^{1}$, Aline Taoum ${ }^{1}$, Pierre Jéhannin ${ }^{2}$, Guillaume Mahé2, Alexis Le Faucheur ${ }^{1}$

${ }^{1}$ University of Rennes, ${ }^{2}$ University Hospital

Introduction: The need of accumulating consecutive physical activity (PA) bouts $\geq 10$ minutes to reach the total weekly dose of moderate to vigorous PA (MVPA) has been removed from recent PA recommendations, suggesting that every PA bout counts, even short ones. Sound assessment of daily-life walking is challenging due to its intermittent nature with high proportion of short $(<1 \mathrm{~min})$ walking bouts. We aimed to compare activity monitors and event-based analysis methods in the estimation of daily-life walking pattern. Methods: 35 healthy older participants wore an ActivPAL (PAL) and a StepWatch3 (SW) for a 7-day free living period. Bout-level analysis methods were defined by the epoch length and the number of consecutive steps or strides to reach to consider a walking bout. SW data strides were processed over 10s-epoch with walking bout $\geq 2$ consecutive strides (SW_2STR_10S) according to Orrendurff et al. (2008). For PAL data, we also implemented the Orendurff method (PAL_2STR_10S), and another one that analysed step count over 1 s epoch with walking bout $\geq 3$ consecutive steps (ie. $\geq 1$ gait cycle, PAL_3STEPS_1S). In addition, PAL acceleration data were processed from the ActivPAL software (VANE algorithm) over 1s-epoch (PAL_VANE_1S). Bouts frequency and total walking time for different incremental walking duration bands were reported. The total walking time between methods or devices was compared using a repeated measures ANOVA. Results: The 11-20s walking bout durations were those that most frequently occurred when using the Orendurff method (PAL or SW), against the 1-10s duration band for both PAL_3STEPS_1S $(82 \pm 4.0 \%)$ and PAL_VANE_1S $(61 \% \pm 5.5 \%)$ methods. Total walking time was different between each method, except between SW_2STR_10S and PAL_2STR_10S that tended to show comparable results (Figure 10). Discussion: The use of different bout method analysis can provide a different picture of daily-life walking pattern.

\section{Contextualizing Device-Measured Sitting and Sitting Patterns Among} Older Adults Using Self-Reported Activities

Mikael Anne Greenwood-Hickman ${ }^{1}$, Rod Walker ${ }^{1}$, John Bellettiere ${ }^{2}$, David Wing $^{2}$, Andrea LaCroix ${ }^{2}$, Dori Rosenberg ${ }^{1}$

${ }^{1}$ Kaiser Permanente Washington Health Research Institute, ${ }^{2}$ University of California, San Diego

Introduction: Understanding patterns in the types of activities older adults engage in during sedentary time and how they differ by key demographics and device-based sitting patterns could help shape future intervention designs. Methods: We summarized daily sitting time spent in ten common activities (TV, computer, reading, talking and thinking, transit, hobbies, group activities, napping, eating, and other) for 998 older adults in the Adult Changes in Thought study who wore activPAL for 1 week (age $77 \pm 7.0,57 \%$ female). Differences by activPAL total daily sitting time (> vs. < sample median) and activPAL mean sitting bout duration (> vs. < sample median) described and compared using two sample T-tests. Results: Participants self-reported 10.6 hours $(\mathrm{SD}=4.3)$ daily sitting time and spent the most time watching TV (2.6 hrs/day [1.8]), on the computer (1.7 [1.6]), and reading (1.5 [1.1]). Figure 11 displays time in self-reported sedentary activities by levels of activPAL-based sitting measures. Those with low activPAL-measured sitting time spent significantly less time ( $\mathrm{p}<0.05)$ watching TV ( 2.1 vs. $3.0 \mathrm{hrs} /$ day $)$, using the computer ( 1.5 vs. $2.0 \mathrm{hrs} /$ day), reading ( 1.4 vs. $1.7 \mathrm{hrs} /$ day), and napping ( 0.4 vs. $0.7 \mathrm{hrs} /$ day). Only eating and transit time did not differ between groups. Those with more interrupted activPAL-measured sitting patterns (low mean bout duration) also had significantly less time spent in all activity categories except eating, transit, and group activities. Specifically, the group with the most vs. least interrupted sitting patterns spent less time watching TV ( 2.1 vs. $3.0 \mathrm{hrs} /$ day), using the computer (1.6 vs. $1.8 \mathrm{hrs} /$ day), and reading (1.4 vs. $1.7 \mathrm{hrs} /$ day). Discussion: TV and computer use are common sitting activities for older adults. Those who sit more tend to spend much of their additional sitting time watching TV and on the computer. Future interventions to reduce older adult sitting time may target TV and computer time.

\section{Development of Novel Accelerometry-Based Markers to Identify Performance Fatigability During a Fast-Paced 400m Walk in Older Adults}

Jaroslaw Harezlak ${ }^{1}$, Robert Boudreau ${ }^{2}$, Jacek Urbanek ${ }^{3}$, Kyle Moored ${ }^{2}$, Jennifer Schrack ${ }^{3}$, Eleanor Simonsick ${ }^{4}$, Nancy Glynn ${ }^{2}$

${ }^{1}$ Indiana University, ${ }^{2}$ University of Pittsburgh, ${ }^{3}$ Johns Hopkins University, ${ }^{4}$ National Institute on Aging

Introduction: Slowing down during a standardized physical task is a hallmark of performance fatigability. Current computational strategies to assess performance deterioration rely on lap splits from stopwatches, which are subject to recording errors and may be insensitive to subclinical changes. To overcome these limitations, we developed an analytical approach to extract three potential slow-down markers using accelerometry in older adults. Methods: Higher functioning older adults $(\mathrm{N}=57$, mean age $=79$ years $)$ wore an ActiGraph GT3X+ (sampling $=80 \mathrm{~Hz})$ on non-dominant wrist during a fast-paced $400 \mathrm{~m}$ walk. Raw tri-axial accelerations were first reduced to vector magnitude counts in $1 \mathrm{~s}$ windows to manually identify walk start/stop times. Next, the Adaptive Empirical Pattern Transformation (ADEPT) algorithm was used to estimate stride-tostride gait cadence in sub-second, raw accelerometry data, for each individual separately. Lastly, individual-level smoothed cadence trajectories were estimated with penalized regression splines via "mgcv" $\mathrm{R}$ package. Results: We developed three potential slow-down markers: 1) Time-to-slow-down defined as the first time-point where the first derivative of cadence trajectory up-crosses zero. If no slow-down is detected, marker is censored at walk completion time; 2) Start-to-slowdown defined as the first time-point, before time-to-slow-down, where the first derivative of cadence trajectory down-crosses zero. Marker is censored at zero if participants exhibit persistent slow-down starting immediately at the outset of walk; 3) Duration-of-slow-down defined as time difference between Marker1 and Marker2. Median (IQR) time-to-slowdown, start-to-slow-down and duration-of-slow-down were $2.83 \mathrm{~min}$ (1.45-6.68), $0.58 \mathrm{~min}(0.11-2.18)$ and $1.30 \mathrm{~min}(0.89-1.78)$, respectively. Discussion: Future work will refine and validate these performance fatigability markers and explore their associations with established fatigability measures and functional performance.

\section{Examining the Difference Between Weekend and Weekday Sleeping Patterns of Preschool Aged Children Enrolled in the Guelph Family Health Study (GFHS)}

Bridget Coyle-Asbil ${ }^{1}$, Hannah Coyle-Asbil ${ }^{1}$, David W Ma ${ }^{1}$, Jess Haines ${ }^{1}$, Lori Ann Vallis ${ }^{1}$

${ }^{1}$ University of Guelph

Introduction: Sleep is vital for healthy development; however, it is not clear how quality and quantity of sleep vary between the weekends and weekdays (WE-WD) in young children. Previous research focused on 
physical activity, has proposed that WEs and WDs must be included in analyses to appropriately reflect a child's typical behaviour. Currently no guidelines exist for researchers on whether this is necessary to accurately evaluate sleep in preschoolers. Although plausible, having strict WE/WD criteria could be a major drawback if wear compliance across the week is poor. The objective of this study was to determine whether there are differences in the WE/WD sleep patterns of preschool children, and whether this is experienced similarly by both sexes. Methods: Eight-five preschool aged children (44 M; $4.48 \pm 0.89$ years) enrolled in the GFHS wore an ActiGraph wGT3X-BT accelerometer $(100 \mathrm{~Hz})$ on their right hip for 7 days of 24 hours. WE nights were defined as Friday-Saturday and WD nights were Sunday-Monday. Data was exported in 1-min epochs with the low frequency extension feature enabled. Sleep scoring was performed using the Sadeh et al. (1994) algorithm and the Tudor-Locke et al. (2014) algorithm was applied to identify sleep periods and compute metrics. Total sleep time (TST), sleep efficiency (SE), sleep onset and offset metrics were used to compare sleep timing and quality between WEs and WDs. Results: A two-way ANOVA revealed no significant main effect of Day for any calculated sleep metric, however a significant main effect of Sex was observed for sleep onset $(\mathrm{F}(1,84)=6.695, \mathrm{p}=0.011, \beta=0.730)$ and sleep offset $(F(1,84)=4.966, p=0.027, \beta=0.601)$. In general, males got in and out of bed earlier than females on WEs and WDs. Discussion: Findings demonstrate that sleep inclusion criteria, does not need to be limited by WE/WD constraints in preschool populations, which has positive implications if wear compliance is poor for pediatric populations.

Factors Associated With Physical Activity and Sedentary Time in Older Adults: Results From the Whitehall II Accelerometer Substudy

Mathilde Chen ${ }^{1}$, Vincent van Hees ${ }^{2}$, Manasa Yerramalla ${ }^{1}$, Aline Dugra$\operatorname{vot}^{1}$, Benjamin Landre ${ }^{1}$, Aurore Fayosse ${ }^{1}$, Mohamed Amine Benadjaoud ${ }^{3}$, Séverine Sabia ${ }^{1}$

${ }^{1}$ Inserm, ${ }^{2}$ Amsterdam UMC, ${ }^{3}$ Institute for Radiological Protection and Nuclear Safety

Introduction: Identification of factors associated with older adults' physical activity (PA) and sedentary behavior (SB) is essential to inform effective targeted prevention strategies to prevent the impact of lack of PA and high sedentary time on health in older adults. While most previous studies examined factors associated activity levels separately, this study investigated factors associated with the activity distribution using the continuum of acceleration, avoiding loss of information that could arise from categorization of activity levels. Methods: This study included 3896 participants (25.3\% women, mean [range] age: 69.4 [60-83] years) from the Whitehall II accelerometer sub-study. The association of socio-demographic, lifestyle and cardiometabolic factors with the whole activity intensity distribution and time in SB, light PA and moderate-to-vigorous PA was examined using functional data analysis and linear regressions, respectively. The consistency of the associated factors over time was examined using factors assessed at the time of the activity measure, and 9 and 18 years prior this measure. Results: Cross-sectional analyses showed participants who were older, current smokers, obese, with chronic conditions, and those reporting poor SF36 physical component score spent more time in the lowest acceleration intensities at the expense of less time in higher intensities. Occupational position in men and SF36-mental component score in women were additionally associated with activity distribution. In both men and women, the pattern of factors associated with PA and SB were generally similar in prospective and cross-sectional analyses. Discussion: This study confirms socio-demographic disparities in PA and SB and identify several modifiable factors associated with which are eligible to health promotion interventions. Factors associated with activity intensity distribution at older age were consistent in the 20 years before physical activity measure.
Investigating an Ideal Combination of Time Spent in Physical Activity and Sedentary Behavior That is Associated With Greatest Reduction in Mortality Among Older Women

Jairo Migueles ${ }^{1}$, I-Min Lee ${ }^{2}$, Cristina Cadenas-Sanchez ${ }^{3}$, Francisco Ortega $^{4}$, Julie Buring ${ }^{2}$, Eric Shiroma ${ }^{5}$

${ }^{1}$ Linköping University, ${ }^{2}$ Harvard Medical School, ${ }^{3}$ Public University of Navarra, ${ }^{4}$ University of Granada, ${ }^{5}$ National Institutes of Health

Introduction: While physical activity has been associated with decreased mortality rates, it remains unknown if there is an "ideal" combination of time in physical activities of different intensities and sedentary behavior (SB) associated with the lowest mortality rate. This study examined the associations of combinations of time in moderate-to-vigorous intensity (MVPA), higher-light intensity (HLPA), lower-light intensity activities (LLPA), and SB with mortality rates in older women. Methods: Prospective cohort study of 16,676 US older women from the Women's Health Study. Women wore accelerometers in 2011-2015 and were followed up to 2017. Compositional Cox regression models were performed. Results: The mean (SD) age was 72 (5.7) years; 503 women died. Compared to the least active women (mean, $3 \mathrm{~min} /$ day MVPA, $27 \mathrm{~min} /$ day HLPA, $162 \mathrm{~min} /$ day LLPA, and $701 \mathrm{~min} /$ day SB): compositional models showed an inverse L-shaped dose-response association of MVPA replacing other behaviors with mortality rates $(\mathrm{P}=.02)$; higher SB replacing LLPA, HLPA, and MVPA was associated with higher mortality rates in a curvilinear dose-response manner $(\mathrm{P}<.001)$; replacing $10 \mathrm{~min}$ of SB for MVPA $(\mathrm{HR}=.86)$ or for HLPA $(\mathrm{HR}=.94)$ associated with $14 \%$ and $6 \%$ lower mortality rates, respectively; a $47 \%$ risk reduction $(\mathrm{HR}=.53)$ was observed among women meeting physical activity guidelines (mean, $36 \mathrm{~min} /$ day MVPA, $79 \mathrm{~min} /$ day HLPA, $227 \mathrm{~min} /$ day LLPA and $549 \mathrm{~min} /$ day $\mathrm{SB})$; and similar mortality rate reductions of $43 \%(\mathrm{HR}=$ .57) were observed with increases in HLPA (+90 min/day) and LLPA (+120 min/day) without increasing MVPA (+0 min/day). Discussion: There was no "ideal" combination of physical activities of different intensities and SB associated with the lowest mortality rates. Replacing SB with light intensity activity was associated with lower mortality rates, and "mixing and matching" times in different intensities yielded equivalent mortality risk reductions.

\section{Physical Activity and Sedentary Behaviour in Patients Following Attendance at Exercise Rehabilitation}

Sapna Khusal ${ }^{1}$, Estelle Watson ${ }^{1}$

${ }^{1}$ Department of Exercise Sciences, University of Auckland

Introduction: Following significant health events, exercise rehabilitation is prescribed to assist with increasing physical activity (PA) and reducing sedentary behaviour. People who attend exercise rehabilitation should, by definition, meet the current guidelines of PA required for good health but long-term adherence to exercise and PA is poor. This study determined whether a sample of people who have attended exercise rehabilitation in the past year meet the current recommended PA guidelines. Methods: Men and women $(n=59)$ between the ages of 40 and 80 years, living with a chronic condition wore an activPAL over 24 hours for seven days and qualitatively reported on their change in activity following rehabilitation. Self-efficacy for exercise (1-5 scale) and joint-related pain were measured. Results: Participants took a mean (SD) 8541 (3544) steps and spent 4.2 (1.2) hours standing, 1.8 (0.7) hours stepping and 8.9 (1.8) hours sitting. Participants spent 157.4 (131.0) minutes per week stepping at a cadence $>100$ steps per minute. Only $36.8 \%$ of participants achieved greater than 150 minutes per week of stepping at a cadence of 100 steps per minute while $63.2 \%$ achieved less than that. Twenty five percent of participants reported their activity decreasing, $36.0 \%$ reported that it had increased and 
$22.5 \%$ reported that it had stayed the same following rehabilitation. Participants were at least a little sure that they would engage in exercise in different situations (median (min-max) score: 2.3 (1.7-3.3)). Most respondents $(60 \%)$ reported experiencing knee pain while only $25 \%$ experienced hip pain. Discussion: Contradictory to self-report, less than half the respondents were able to achieve stepping activity at a moderate intensity after exercise rehabilitation. Sedentary behaviour was similar to that previously reported in older adults. Strategies are required following rehabilitation to ensure that participants are motivated to maintain longterm healthy activity behaviours.

Physical Activity Level and Perceived Barriers to Physical Activity Participation Among Nurses at the University College Hospital, Ibadan, Nigeria

Ayodeji Fabunmi ${ }^{1}$, Oluwafunmilayo Kajero ${ }^{1}$

${ }^{1}$ University of Ibadan

Introduction: Evidence has shown that regular physical activity of moderate to vigorous intensity has health benefits. Among health care professionals Nurses represent the largest group of health care professionals. The aim of this study is to determine physical activity level and perceived barriers to physical activity participation among nurses at the University college Hospital, Ibadan. Methods: This research is cross sectional survey design. Ethical approval was obtained before the study commence with number UI/EC/16/ 0194. All willing nurses were selected to participate in this study. International Physical Activity Questionnaire (IPAQ-SF) was used to collect information on physical activity level of participants. The Physical Activity Barriers (PAB) questionnaire was used to collect information on the perceived barriers to physical activity participation. Data were analysed using descriptive statistics (mean, standard deviation, frequencies, percentages and pie charts). Inferential statistics (anova) was used to test the difference in barriers to physical activity across the nursing rank. The level of significance was set at 0.05. Results: Out of 230 participants, $56(24.3 \%)$ had high physical activity level while $100(43.5 \%)$ and 74 (32.2\%) had moderate and low PAL respectively. "Lack of energy after work" (34.8\%) was the most frequently reported personal barrier, "lack of free time" (39.1\%) was the most frequently reported social environmental barrier while "absence of facilities or places to do PA" $(24.3 \%)$ was the most frequently reported physical environmental barrier. There was significant difference in social and physical environment factor as a limitation to PA across the nursing rank. Discussion: Participants in this study were females. Lack of time, taking care of the family and lack of energy were the frequently reported barriers to PA.

Physical Activity Patterns in People With Pre- and Type 2 Diabetes: A Latent Class Analysis of Longitudinal Data

Philip von Rosen ${ }^{1}$, Jenny Rossen ${ }^{2}$, Maria Hagströmer ${ }^{1}$

${ }^{1}$ Karolinska Institutet, ${ }^{2}$ Sophiahemmet University

Introduction: Few studies have identified physical activity (PA) profiles based on longitudinal data. Insight into how PA pattern change during interventions and among subgroups is required to understand predictors for behavior change. The primary aim of this study was to classify individuals with pre- and type 2 diabetes, participating in a two-year randomized controlled PA trial, based on their change in PA patterns across the trial. The secondary aim was to identify predictors associated with different PA patterns. Methods: All participants $(n=166)$ participated in the Sophia Step Study, a randomized controlled PA trial. PA and sedentary behaviour (SB) were assessed with an ActiGraph GT1M accelerometer at baseline, 6 and 12 months. A total of $15 \mathrm{PA}$ and SB variables were entered into a latent class mixed model for multivariate longitudinal outcomes and a multinominal regression analysis was conducted to model profile membership.
Results: Three different PA profiles were identified. Profile 1, Negative responders" ( $n=47,28 \%)$, reflects participants that reported adverse values for PA and SB, while Profile 2, "Responders" $(\mathrm{n}=22,13 \%)$, increased time spent in PA and decreased time in SB across time. Profile 3, "Maintainers" ( $\mathrm{n}=97,58 \%)$, reported minor changes for PA and SB across time. The multinomial logistic regression analysis showed BMI was significantly $(\mathrm{p}<0.05)$ associated with profile membership, where higher BMI values decreased the odds of belonging in the "Responders" and "Maintainers" profiles. Allocation to the intervention or control group was not associated with profile membership. Discussion: The majority of participants maintained their PA and SB pattern over 12 months and less than a third of all participants showed an unfavourable PA pattern. BMI was the only variable that predicted a beneficial PA pattern.

Relationship Between Selected Psychological Factors and Physical Activity Among Undergraduates, University of Ibadan. Nigeria

Ayodeji Fabunmi ${ }^{1}$, Esther Uzokife ${ }^{1}$

${ }^{1}$ University of Ibadan

Introduction: The purpose of this study was to assess the relationship between exercise self- efficacy, exercise self- motivation and physical activity among undergraduates. Methods: This study was a cross- sectional study. Participants were selected using random sampling technique from 10 halls of residence. Socio- demographic variables (age, gender and BMI) were obtained using a socio- demographic form, the physical activity level of the participants was assessed using the International Physical Activity Questionnaire Short Form (IPAQ- SF), exercise self- efficacy was assessed using the Self- Efficacy for Exercise Scale (SEES) and exercise selfmotivation was assessed using Exercise Motivations Inventory (EMI-2). Descriptive statistics of mean, standard deviation, frequency and pie charts were used to present data. Spearman's rank- order correlation was used to determine the relationship between exercise self- efficacy, exercise selfmotivation and physical activity. Alpha level was set at 0.05 . Results: 400 undergraduate students with age range between 16-33 years and mean age of $20.40 \pm 2.30$ years. BMI ranged from $16.26-40.07 \mathrm{~kg} / \mathrm{m}^{2}$ with a mean BMI of $21.65 \pm 2.91 \mathrm{~kg} / \mathrm{m}^{2}$. $50.5 \%$ of participants were moderately physically activity, $30.0 \%$ were involved in low physical activity, and $19.5 \%$ were involved in high physical activity. $63.2 \%$ of participants had moderate exercise self- efficacy, $31.0 \%$ had low exercise self- efficacy and $5.8 \%$ had high exercise self- efficacy. $71.8 \%$ of participants had moderate exercise self- motivation, $21.2 \%$ had low exercise self- motivation, and $7.0 \%$ had high exercise self-motivation. There was a significant relationship between exercise self- efficacy $(p=0.000 \mathrm{r}=0.194)$, exercise selfmotivation $(p=0.000 r=0.220)$ and physical activity. Discussion: The outcome of this study showed that participants with higher exercise selfefficacy and self- motivation had higher physical activity levels.

Relative Importance of Overall Activity and Intensity of Physical Activity for Cardiometabolic Risk in Adults With and Without a Chronic Condition

Nathan Dawkins ${ }^{1}$, Tom Yates $^{1}$, Charlotte Edwardson ${ }^{1}$, Ben maylor ${ }^{1}$, Melanie Davies ${ }^{1}$, David Dunstan ${ }^{2}$, Patrick Highton ${ }^{1}$, Louisa Herring ${ }^{1}$, Kamlesh Khunti ${ }^{1}$, Aelx Rowlands ${ }^{1}$

${ }^{1}$ University Of Leicester, ${ }^{2}$ Baker Heart and Diabetes Institute

Introduction: Higher levels of physical activity are associated with lower cardiometabolic risk. However, the relative contribution of overall activity and the intensity of activity is unknown. Our aim was to determine the relative contribution of overall activity and intensity distribution of activity to cardiometabolic risk in ostensibly healthy office workers and in people with one or more chronic disease. Methods: Clustered cardiometabolic risk score 
was calculated from mean arterial pressure, HDL cholesterol, triglycerides and HbAlc. Open source software (GGIR) was used to generate average acceleration and intensity gradient from wrist-worn accelerometer data for two datasets: office-workers who did not have a self-reported medical condition $(\mathrm{N}=399)$ and adults with $\geq 1$ chronic disease $(\mathrm{N}=1,137)$. A series of multiple linear regressions were used to assess the relative contribution of overall activity and intensity of activity to cardiometabolic risk. Results: When mutually adjusted for the other, both overall activity and intensity of activity were associated with cardiometabolic risk in the office workers. However, only overall activity was associated with cardiometabolic risk for the chronic disease group. Office workers with the lowest cardiometabolic risk typically undertook higher amounts of overall activity and/or higher intensity of activity. Those undertaking the highest amounts of overall activity in the chronic disease group had the lowest cardiometabolic risk irrespective of the intensity of the activity. Discussion: The office workers were more active than adults with chronic disease and had better health indicators. These findings are cross-sectional but suggest that interventions aiming to benefit cardiometabolic health in healthy adults could focus on increasing either intensity or amount of physical activity. However, in those with chronic disease increasing the amount of activity undertaken, irrespective of intensity, may be more appropriate.

\section{Sleep Parameters and Accelerometry: A Comparison Between Com- mon Definitions}

Kim Meredith-Jones ${ }^{1}$, Rachael Taylor ${ }^{1}$

${ }^{1}$ University of Otago

Introduction: Accelerometry is increasingly used to estimate sleep. To do this, each minute is automatically scored as sleep or wake from the actigraphy data. Sleep-wake data are then separately processed using automated algorithms to estimate a range of sleep parameters. Details describing the methodology for the derivation of sleep parameters is lacking, and reporting of sleep parameters is inconsistent, likely reflecting the lack of consensus regarding the definitions for typical sleep parameters. This study aims to demonstrate the differences between sleep parameter definitions, highlighting the difficulty of comparing results derived using different algorithms. Methods: Five widely used sleep parameter definitions (total sleep time (TST), sleep onset latency (SOL), wake after sleep onset (WASO), sleep efficiency (SE), number of awakenings) will be detailed. Results: TST definitions vary widely including: 1) total number of epochs scored asleep between time in bed (TIB) and time out of bed (TOB), 2) total duration of sleep periods between TIB and TOB 3) total number of epochs scored as sleep between sleep onset to offset, and 4) total duration of sleep periods between sleep onset to offset. WASO definitions include: 1) number of epochs scored awake between TIB and TOB, 2) number of epochs scored awake between sleep onset to offset, 3) duration of all awakenings between TIB and TOB, and 4) duration of all awakenings between sleep onset to offset. SOL definitions also vary including 1) time to first sleep epoch, and 2) time to start of sleep period. SE definitions were the most variable, including 1) \% epochs scored awake between TIB and TOB, 2) \% epochs scored awake between onset to offset, 3) $\%$ sum of all awakenings in minutes between TIB and TOB, and 4) $\%$ sum of all awakenings in minutes between onset to offset. Discussion: Improving understanding of the actigraphic process may ensure appropriate use and interpretation of sleep parameters in the future.

The Effect of Consumer-Based Activity Tracker Intervention on Physical Activity Among Recent Retirees: The REACT Trial

Tuija Leskinen ${ }^{1}$, Jesse Pasanen ${ }^{1}$, Kristin Suorsa ${ }^{1}$, Ilkka Heinonen ${ }^{1}$, Sari Stenholm ${ }^{1}$

${ }^{1}$ University of Turku
Introduction: Majority of older adults do not meet the physical activity recommendations highlighting the need for new, scalable strategies to promote physical activity among older population. The REACT trial (NCT03320746) examined the effect of a 12-month consumer-based activity tracker intervention on accelerometer-measured physical activity among recent retirees. Methods: Overall, 231 recently retired Finnish adults (65.2 (SD 1.1) yrs, 83\% women) were randomized into intervention and control groups. Intervention participants were requested to wear a commercial wrist-worn activity tracker (Polar Loop 2, Finland) for 12 months and to try to reach the activity goal shown on the tracker's display every day. The control group received no intervention. Physical activity was measured using a wrist-worn ActiGraph wGT3X-BT at baseline, 3-, 6, and 12-months. The 24-h accelerometer data were analyzed using the $\mathrm{R}$ package GGIR. Wake-time light (LPA) and moderate-to-vigorous physical activity (MVPA) were determined using threshold values of $\geq 30.0 \mathrm{mg}$ and $\geq 100.6 \mathrm{mg}$, respectively, and total physical activity as their sum. Hierarchical linear mixed models adjusted for accelerometer's wake wear time were used to examine the differences between the groups. Compositional data analysis will be conducted to examine simultaneous changes in all 24$\mathrm{h}$ movement behavior components and results will be presented at the conference. Results: The use of the commercial activity tracker did not change total, LPA or MVPA over the 12 months compared to the non-user controls (group*time interaction p 0.39, 0.23, 0.77, respectively). The short-term increase in total and LPA over the first 6 months favored intervention, but the differences in the changes between the groups were not significant (11 $\mathrm{min} / \mathrm{day}, 95 \% \mathrm{CI}-9$ to 31 and $12 \mathrm{~min} / \mathrm{day}, 95 \% \mathrm{CI}-6$ to 30, respectively). Discussion: Long-term use of a commercial activity tracker does not promote physical activity among a general population recent retirees (Figure 12).

\section{The Effect of Tailored Feedback on Daily Step Count Over 6 Months: A Longitudinal Study}

Sarah McGarry ${ }^{1}$, Joanne McVeigh ${ }^{1}$, Hannah Baker ${ }^{1}$, Emily Hoops ${ }^{1}$, Naomi Jones ${ }^{1}$, Rhiannon Halse ${ }^{1}$, Leon Straker ${ }^{1}$, Deborah Kerr ${ }^{1}$

\section{${ }^{1}$ Curtin University}

Introduction: Many adults are not achieving the recommended 10,000 steps per day which can negatively impact physical and mental health, particularly for those with a body mass index (BMI) $>25 \mathrm{~kg} / \mathrm{m} 2$. This study investigated whether tailored feedback increased daily step counts in adults with a BMI $>25 \mathrm{~kg} / \mathrm{m} 2$ over a six-month period. Methods: This was a substudy of a randomised controlled trial and included participants aged 18 to 65 years with a BMI $>25$ to $<40 \mathrm{~kg} / \mathrm{m} 2$. Participants wore a Fitbit Charge 2 (®) for six-months and received tailored feedback at six time points. A mixed-model repeated measure was used to assess the effect of tailored feedback on change in step count at each time point. Associations between gender, step count and categorical response to feedback (whether step count was maintained or not) were analysed. Results: Sixty-six participants were included (65\% female; age (mean (SD)) 48.4 years (10.7); BMI $31.2 \mathrm{~kg} / \mathrm{m} 2(4.1))$. Males had significantly increased step counts in the week following feedback at time point two (mean difference $(95 \% \mathrm{CI}$ ): 1184 (169 to 2130) steps/day, $\mathrm{p}=0.025$ ). Females had significantly increased step counts in the week following feedback at time point three (1077 (18 to 2135 ) steps/day, $\mathrm{p}=0.046$ ). Males had significantly higher step counts compared to females in all post-feedback weeks, except for time point three. Non-maintainers had significantly higher depression scores $(p=0.023)$ than maintainers. Discussion: The findings suggest that tailored feedback can be effective in maintaining daily step counts in people with a BMI $>25 \mathrm{~kg} / \mathrm{m} 2$ over six-months. Health-based programs aiming to change PA behaviour require feedback to be tailored to gender, performance, and motivational impacts such as depression. Future research 
is required to develop effective step count programs to assist females globally in reaching the recommended PA guidelines.

\section{The Relation Between Accelerometer-Derived Physical Activity and} Brain Structure: A Population-Based Study

Fabienne A Fox ${ }^{1}$, Kersten Diers ${ }^{1}$, Hweeling Lee ${ }^{1}$, Martin Reuter ${ }^{1}$, Monique M Breteler ${ }^{1}$, N Ahmad Aziz ${ }^{1}$

${ }^{1}$ German Center for Neurodegenerative Diseases

Introduction: Physical activity promotes neuronal health in animals but human studies examining the relation between PA and brain health are inconclusive. We therefore assessed whether objectively-quantified PA is associated with brain structure in a large cohort study. Methods: The source population consisted of the first 5000 participants of the Rhineland Study, a prospective cohort study in Bonn (Germany). We included data of 2649 participants (57.6\% female, mean age: 54.7 years, range: $30-94)$ with valid PA and MRI measurements. Metabolic equivalent (MET)-hours, step count and minutes spent stepping as well as time spent performing sedentary, light-intensity and moderate-to-vigorous physical activities were continuously recorded over 7 days with accelerometers. Brain volumetric and cortical thickness measures were obtained from 3T MRI scans using FreeSurfer. The relation of PA (independent variable) and brain structure (outcome) was examined with polynomial multivariable regression while adjusting for age, sex, eTIV, education and smoking. Results: MET-hours were associated with greater grey matter volume and precentral thickness. Whereas total brain and grey matter volume increased with more light-intensity activities (Figure 13A), lateral-occipital thickness decreased (Figure 13B). With increasing age, light-intensity activities displayed a stronger effect on total brain volume, but a weaker effect on lateral-occipital volume (Figure $13 \mathrm{C} \& \mathrm{D}$ ). Vertex-wise analysis demonstrated that higher PA levels were related to larger cortical thickness in frontal and temporal areas, but lower thickness in parietal and occipital areas. Discussion: PA was associated with several volumetric measures and cortical thickness, but its effect was not uniform across the brain. The effect of PA on brain volume accelerated at low PA levels and decelerated at high levels. PA may particularly benefit motor regions and could be critical in the prevention of age-associated brain atrophy.

Time Trends in Physical Activity Using Wearable Devices: Systematic Review and Meta-Analysis of Studies in Children, Adolescents, and Adults, 1995-2017

Scott Conger ${ }^{1}$, Lindsay Toth ${ }^{2}$, Channie Cretsinger ${ }^{3}$, Anders Raustorp ${ }^{4}$, Josef Mitás ${ }^{5}$, Shigeru Inoue ${ }^{6}$, David Bassett ${ }^{3}$

${ }^{1}$ Boise State University, ${ }^{2}$ University of North Florida, ${ }^{3}$ University of Tennessee, ${ }^{4}$ University of Gothenburg, ${ }^{5}$ Palacký University, ${ }^{6}$ Tokyo Medical University

Introduction: There is conflicting evidence on whether physical activity (PA) levels of humans have changed over the last quarter-century. The main objective of this study was to determine if there is evidence of time trends in PA, from cross-sectional studies that assessed PA at different time points using wearable devices (e.g., pedometers, accelerometers). A secondary objective was to quantify the rate of change in PA. Methods: A systematic literature review was conducted of English language studies indexed in PubMed, SPORTDiscus, and Web of Science (1960-2020) using search terms (time OR temporal OR secular) AND trends AND (steps per day OR pedometer OR accelerometer OR MVPA). Subsequently, a meta-analytic approach was used to determine if there was an overall time trend in ambulatory PA, expressed as steps per day or counts per minute. Sub-group analyses were conducted to answer this question in subgroups based on sex, age group [children, adolescents, adults], sex-and-age group, and PA assessment method [steps or CPM]. Results: Based on 16 peer-reviewed scientific studies conducted between 1995 and 2017, levels of ambulatory PA are trending downward in developed countries. Significant declines were seen in both males $(\mathrm{p}=0.015)$ and females $(\mathrm{p}=0.001)$. The decline in PA was seen most strongly in adolescents $(p<0.001)$, but it also approached statistical significance in adults $(p=0.089)$. For studies reporting steps/day, the overall rate of decline was approximately 834 steps/day per decade in all participants, and 1988 steps/day per decade in adolescents. Discussion: Evidence from studies conducted in eight developed nations over the last 25 years indicates that PA levels have declined. Declines in PA may have contributed to increases in the prevalence of obesity and chronic, non-communicable diseases.

\section{Applications: Healthy Environments}

Identifying Digital Biomarkers of Mobility During Daily Living in People Recovering From Mild Traumatic Brain Injury

Kody Campbell ${ }^{1}$, Martina Mancini ${ }^{1}$, Laurie King ${ }^{1}$

${ }^{1}$ Oregon Health and Science University

Introduction: Instrumented laboratory-based mobility assessments provide objective measures of function after mild traumatic brain injury (mTBI) that can be overlooked. Instrumented laboratory-based mobility tests provide only a snapshot of true function and may not adequately reflect mobility during daily living. Passive continuous monitoring may provide a more complete assessment of mobility. However, there is no evidence on which passive monitoring variables would be most sensitive for identifying impairments following mTBI. Here, we investigate which measures of passive monitoring differentiate people with mTBI from healthy controls. Methods: Participants recovering from $\mathrm{mTBI}(\mathrm{N}=30$; age $=37 \pm 12 \mathrm{y})$, and healthy controls $(\mathrm{N}=21$; age $=33 \pm 10 \mathrm{y})$ wore an inertial sensor around their waist for at least 5 continuous hours a day for at least 5 consecutive days. A previously validated algorithm processed raw inertial data to identify bouts of activity, and calculated metrics of turning and activity (Table 1). Area under the curve (AUC) from receiver operating characteristic curves and independent samples tests determined which metrics of passive monitoring differentiated groups. Results: Number of turns/hour and peak turn velocity variability had the highest AUCs among the turn metrics (Figure 14), although still $<0.70$. Among activity metrics, the average number of daily bouts had the highest AUC but was also $<0.70$. Turn duration and peak turn velocity variabilities, as well as average number of daily bouts significantly differed between groups (Table 1). Discussion: Although no single turn or activity metric showed a high AUC in discriminating between groups, people with mTBI showed lower turning duration and velocity variability as well as higher numbers of turns/hour. Future studies will use logistic regression models using 'best subsets selection strategy' to find combinations of turning and gait metrics that discriminate mobility in healthy controls and people with mTBI.

Park Availability and Physical Activity Among Children and Adolescents: Findings From the Healthy Communities Study

Matthew Stewart ${ }^{1}$, Manish Verma ${ }^{1}$, Alisha Rajbhandari ${ }^{2}$, Cathy Antona$\operatorname{kos}^{1}$, Natalie Colabianchi ${ }^{1}$

${ }^{1}$ University of Michigan, ${ }^{2}$ Battelle Memorial Institute

Introduction: There is a national initiative to provide every American access to a park within a 10-minute walk of the home. This study examined the association between park density within $1 \mathrm{~km}$ of a child's residence with self-reported park-specific physical activity and accelerometer-derived MVPA in a national sample of $\mathrm{K}$ through 8 th grade youth. Methods: A subsample $(\mathrm{n}=707)$ of youth from the Healthy Communities Study (HCS, $\mathrm{n}=5,138$ ) completed an enhanced protocol that included self- 
report and objective PA data. Self-report PA data was transformed into a binary outcome to identify whether participants had been active in a park in the last 24 hours. MVPA was captured using the ActiGraph GT3X+. Park density was the proportion of park land in a $1 \mathrm{~km}$ Euclidean buffer around the participant's residence, to which an arcsine transformation was applied. Covariates included age, sex, ethnicity, and family income. Analysis consisted of logistic and linear regression modeling with interaction effects. The analytic sample consisted of 493 youth with complete data. Results: Increasing park density was associated with greater self-reported physical activity at parks in the model with covariates (OR $=4.49 ; 95 \%$ CI: $1.35,18.29)$ and without covariates (OR $=4.82$; 95\% CI: 1.24, 18.71). Covariates were unrelated to park-specific PA. Accelerometer analysis revealed an active sample (median $=49.2$ minutes, range $=0.3-195.9$ ). MVPA was unrelated to park density, though older children $(\mathrm{OR}=-0.63$; 95\% CI: $-0.71,-0.56)$ and girls (OR $=-0.99$; 95\% CI: $-1.31,-0.66$ ) engaged in less MVPA. Interactions were not statistically significant. Discussion: Increasing park density may improve youth physical activity patterns. As the relationship between park density and physical activity was present only when examining context-specific physical activity, future research should further examine the relationship between parks and objectively-assessed physical activity using multi-sensor devices.

\section{Park Features and Physical Activity Among Low-Income and Racial/ Ethnic Diverse Children}

Scott Ogletree ${ }^{1}$, Claudia Alberico ${ }^{1}$, Myron Floyd ${ }^{1}$, Oriol Marquet ${ }^{2}$, JingHuei Huang ${ }^{1}$, J. Aaron Hipp ${ }^{1}$

${ }^{1}$ North Carolina State University, ${ }^{2}$ Universitat Autònoma de Barcelona

Introduction: This specific study aims to examine the types of park features where children are most active within park space. Understanding the types of park features associated with physical activity (PA) for children in lowincome, ethnically/racially diverse neighborhoods can be important to creating spaces that maximize the possibility of physical activity. Methods: Six parks in New York City, NY, and six in Raleigh-Durham, NC, were chosen to focus on the target population of children 5-10 years old $(n=357)$. The parks were selected in order to target neighborhoods with low-income, ethnic/racial diverse populations. Data collection occurred in spring and summer of 2017 and 2018. Parents and their children were intercepted and the children asked to wear accelerometers (Actigraph GT3X+) and GPS (QStarz BT1000XT) during park visits for a minimum of 15 minutes. The accelerometer and GPS data were joined at 15-second epochs, mapped and aggregated to defined park feature areas (e.g., basketball court, playground set, etc.). Results: Different park features were associated with different levels of MVPA by subgroup. For African American children the greatest percentage of MVPA was found in play areas like playgrounds or water features (40\%). For Latino children play courts, like ball courts, had the highest percentage of MVPA (46\%). For Asian children it was in play fields (45\%). Also of interest was the amount of time spent between defined park features. Children spent $35 \%$ of the monitored time in-between features, such as walking between a basketball court and swing set. When in-between, children were in MVPA $28 \%$ of the time. Discussion: For lower-income and diverse children in parks in low-income, diverse neighborhoods, including features like play courts and playgrounds with active play equipment can be a means of encouraging PA and well being. Additionally, when assessing children's PA in parks, in-between space must be furthered considered.

\section{Standing Tutorial Meetings in Higher Education}

H.Q. Chim ${ }^{1}$, Renate de Groot ${ }^{2}$, Pascal Van Gerven ${ }^{1}$, Mirjam oude Egbrink $^{1}$, Roy Erkens ${ }^{1}$, Hans Savelberg ${ }^{1}$

${ }^{1}$ Maastricht University, ${ }^{2}$ Open Universiteit
Introduction: With extensive research emphasizing the health risks of prolonged sedentary behavior (SB), we explored the effects of standing versus sitting in university tutorial group meetings on overall physical activity behavior (PAB) and learning. In this exploratory, longitudinal, and randomized controlled trial, 96 first-year students were randomly allocated to a Sit/Stand group, with 2-hour tutorial group meetings scheduled, once/ twice per week, for nine weeks. Methods: PAB was measured using the activPAL3 triaxial activity monitor, analyzed with generalized linear mixed models. Learning was evaluated using exam grades, audio-recorded tutorial interactions, and concept maps. Mann-Whitney U test was used to compare exam grades between groups. The tutorial interactions were coded as learning-oriented interactions (exploratory questioning, cumulative reasoning, or handling of conflict) or non-learning-oriented interactions (procedural or off-task). Marginal models and factorial ANOVAs were used to analyze the concept maps' data. Results: Both on tutorial and non-tutorial days in week $4-5$, the stand group $(n=41)$ showed less SB $(\beta=-0.092$, SE $=0.044,95 \% \mathrm{CI}:-0.179,-0.006)$ and more moderateto-vigorous physical activity $(\beta=0.320, \mathrm{SE}=0.160,95 \% \mathrm{CI}: 0.004$, $0.635)$ compared to the sit group $(\mathrm{n}=36)$. On tutorial days, the stand group showed more LPA than the sit group $(\mathrm{p}<.001, \mathrm{~d}=1.04)$. The average exam grade of the Stand group $(6.5 \pm 1.6$, on a 10-point scale) was higher than the Sit group $(5.8 \pm 1.6)$, but the difference only approached significance, $\mathrm{U}=1130.0, \mathrm{z}=1.80, \mathrm{p}=.071, \mathrm{r}=.20$. No other group difference was observed for learning. Discussion: To conclude, offering standing tutorial group meetings to university students is a recommended solution to break up prolonged SB and encourage more physical activity, while maintaining the learning performance of students.

\section{Walkable Neighborhood During an Intervention Focus on Physical Activity Promotion}

Antoni Colom ${ }^{1}$

${ }^{1}$ Health Research Institute of the Balearic Island (IdISBa). University Hospital Son Espases

Introduction: While urban built environments might promote active aging, an infrequently studied question is how the neighborhood walkability modulates physical activity changes during a physical activity intervention program. We assessed the influence of objectively assessed neighborhood walkability on the change in physical activity during the PREDIMED-Plus intervention program. Methods: The present study involved 228 PREDIMED-Plus senior participants aged between 55 to 75, recruited in Palma de Mallorca (Spain). Overweight/obese older adults with metabolic syndrome were randomized to an intensive weight-loss lifestyle intervention or a control group (106 intervention and 122 control groups). A home base neighborhood environment walkability index (residential density, land use mix, intersections density) was calculated using geographic information systems (1km sausage-network buffer). Physical activity was assessed using the accelerometer for seven days, and a REGICOR validated physical activity questionnaire at baseline and 2 follow-up visits (six-months and one-year later). Generalized Additive Mixed Models (GAMMs) were fitted to estimate the association between the neighborhood walkability index and physical activity changes during follow-up. Results: Higher neighbourhood walkability ( $1 \mathrm{z}$-score increment) was associated with moderate-to-vigorous accelerometer assessed physical activity duration, $(\beta=3,44 ; 95 \% \mathrm{CI}=$ 0.52;6.36 minutes per day). When analyses were stratified by intervention arm, the association was only observed in the intervention group $(B=6.357 ; 95 \% \mathrm{CI}=2.07 ; 10.64$ minutes per day $)(\mathrm{p}$ for interaction $=$ $0.055)$. There were no statistically significant associations between neighborhood walkability and self-reported physical activity. Discussion: The results indicate that the neighborhood's walkability could support a physical activity intervention, helping maintain or increase older adults' objectively measured physical activity. 


\section{Applications: Sports}

\section{Effects of Acoustically Paced Cadence Modulation on Impact Forces in Running}

Anouk Nijs ${ }^{1}$, Melvyn Roerdink ${ }^{1}$, Peter Beek ${ }^{1}$

${ }^{1}$ Vrije Universiteit Amsterdam

Introduction: Increasing cadence in running has been advocated as a means to improve performance and reduce injury risk through lower impact forces. Although acoustic pacing can be used for this purpose, it might by itself lead to increased impact forces. If so, this would counteract the decrease in impact forces pursued by increasing cadence with the help of acoustic pacing and thus have a counterproductive effect. The aim of the study was therefore to assess the effects of acoustic pacing and cadence on peak impact force and loading rate during running. Method: Unpublished data from a previous study, in which 16 participants ran on an instrumented treadmill with various forms of acoustic pacing, were analyzed to address the research question. Peak impact force (IP) and loading rate (LR) while running at three different cadences with and without pacing were extracted from the ground reaction force data and compared statistically between these two main conditions. In addition, we compared step-based and stridebased pacing because these forms may have different effects on the peak impact force and loading rate. Results: As expected, increasing the cadence was accompanied by a significant reduction in peak impact force $(\mathrm{F}(2,24)=8.26, \mathrm{p}=0.002, \eta \mathrm{p} 2=0.41)$ and loading rate $(\mathrm{F}(2,24)=$ $8.08, \mathrm{p}=0.002, \eta \mathrm{p} 2=0.40$ ), whereas acoustic pacing had no significant effect on the impact forces compared to unpaced running with similar cadence, both before and after pacing (IP: $\mathrm{F}(2,28)=0.10$, ns; LR: $\mathrm{F}(2,28)=0.02$, ns) There were also no significant differences in this regard between step-based and stride-based pacing (IP: $\mathrm{F}(2,18)=0.34$, ns; LR: $\mathrm{F}(2,18)=1.17, \mathrm{~ns})$. Discussion: Acoustic pacing does not adversely affect impact force when used to increase cadence in running with the aim of reducing the impact force and can thus be used for this purpose without introducing a counterproductive effect.

Functional Scores Improvement After 6-Month of an Exercise Program for Women With Osteoporosis: A Randomized Trial

Laura Bragonzoni ${ }^{1}$, Erika Pinelli ${ }^{1}$, Giuseppe Audino ${ }^{1}$, Claudio Ripamonti $^{2}$, Francesco Benvenuti, Laura Dallolio ${ }^{1}$, Sofia Marini ${ }^{1}$, Pasqualino Maietta Latessa ${ }^{1}$, Raffaele Zinno ${ }^{1}$, Giuseppe Barone ${ }^{1}$

${ }^{1}$ University of Bologna, ${ }^{2}$ Istituto Ortopedico Rizzoli

Introduction: Osteoporosis (OP) is a systemic disease of the skeleton characterized by a reduced bone mass. There is a consensus on the effectiveness of physical activity in preventing bone loss, falls and, fractures. The main aim of the study is to evaluate modifications of quality of life (QoL). The second aims concern functional score. Methods: Forty women with OP were enrolled in the randomized trial. Women were divided into 2 groups: OGT and IHT. Both groups performed a specific exercise protocol for 6-month in 2-days/week 1-hour sessions. Moreover, all women were requested to choose an additional third day of the week. The OGT was followed by a trainer through online supervision, while the IHT performed the protocol at home without supervision. The participants were assessed at the baseline and after 6-month of training. The assessed outcomes were: QoL measured through ECOS-16, fear of fall by short FES-I, aerobic capacity and endurance assessed by 6MWT, joints mobility by sit and reach and stick test, muscles force by handgrip and 30 seconds chair stand test. Results: ECOS-16 showed no changes at 6-month followup for both groups and no difference between groups. 6MWT and 30s chair stand test showed a statistically significant improvement in both groups. The distance travelled of the 6MWT changed in the OGT, from $420 \pm 25 \mathrm{~m}$ to $457 \pm 46 \mathrm{~m}$ ( $\mathrm{p}<0.005)$, and from $369 \pm 85 \mathrm{~m}$ to $415 \pm 66 \mathrm{~m}(\mathrm{p}<0.05)$ in the IHT. The repetition number of the 30 s chair stand test changed in the OGT from $12 \pm 3$ to $15 \pm 3(\mathrm{p}<0.001)$ and from $12 \pm 2$ to $13 \pm 3$ $(\mathrm{p}<0.005)$ in the IHT. Sit and reach and stick test showed not a significant improvement. Discussion: Despite the difficulty due to the SARS Covid-2 pandemic, the study results showed that participants had maintained the same quality of life after 6-month of training. Moreover, the functional outcomes have increased after the training. These results proved the efficacy of the exercise training in both settings.

\section{Objectively Measured Physical Activity - Weekly Pattern of 2-6-Year- Old Children Over Time}

Linnea Bergqvist-Norén ${ }^{1}$, Emilia Hagman ${ }^{1}$, Claude Marcus ${ }^{1}$, Maria Hagströmer $^{1}$

\section{${ }^{1}$ Karolinska Institutet}

Introduction: The knowledge about physical activity (PA) patterns among children under the age of 6 is scarce. The aim of this longitudinal study was therefore to explore objectively measured physical activity weekly patterns in 2-6-year-old children. Methods: In total, 106 (52\% girls) children, part of the Early STockholm Obesity Prevention Project, had PA measured at least twice between the ages 2-6 years. PA was measured with a tri-axial accelerometer (ActiGraph GT3X+) worn on the non-dominant wrist for seven consecutive days. The main outcome was average PA expressed as counts per minute (cpm) from the vector magnitude. To evaluate differences between days of week factorial repeated analysis of variance (ANOVA) was performed and to investigate differences between weekdays and weekend days paired t-tests were performed. Results: Number of valid days of PA was high and varied from minimum 6.6 to maximum 6.8 for all years. Differences between weekdays were not seen at any timepoint. However, differences between weekdays and weekend days, with higher PA during weekend days, were seen at all years except at age 2 $(\mathrm{p}=.012-<.001)$ these differences became greater over time (Figure 15). Overall, the total PA increased every year from a mean of $3188 \mathrm{cpm}$ at age 2 to a mean of $4392 \mathrm{cpm}$ at age 6. Discussion: 3-6-year-old children have an uneven spread of PA over the week. This finding is important to acknowledge and take into consideration when attempting to affect preschool children's PA. The finding that PA increased over time might not be surprising since the children become more motorically developed. Even so, keeping this in mind when designing interventions involving child PA could result in better fitted interventions.

\section{Postural Control and Proprioception in Women With Osteoporosis Before and After an Exercise Training}

Giuseppe Barone ${ }^{1}$, Erika Pinelli ${ }^{1}$, Maria Grazia Benedetti ${ }^{2}$, Raffaele Zinno $^{1}$, Giuseppe Audino ${ }^{1}$, Laura Bragonzoni ${ }^{1}$

${ }^{1}$ University of Bologna, ${ }^{2}$ Istituto Ortopedico Rizzoli

Introduction: Osteoporosis (OP) is a skeleton systemic disease characterized by a reduced bone mass. OP is accompanied by an increased risk of fall and consequently a high risk of fracture. Since postural control and proprioception are two of the most significant factors in falls prevention, specific balance and proprioceptive exercises could be the keys to reducing the risk of fall and fractures. This study aimed to assess proprioception and postural control, using Delos Postural Proprioceptive System (DPPS; Delos, Turin, Italy), in persons with osteoporosis, before and after an exercise training. Methods: A cohort of 29 women with osteoporosis aged $66.20 \pm 5.80$ was involved in the study. The participants will perform training, structured in 2-days per week, to improve joint mobility, muscle force and balance. Moreover, an additional aerobic activity was requested to improve endurance. Every six weeks, the trainer upgraded the exercise program. DPPS was used to assess postural control and proprioception in 
single-limb stance, respectively, with open and closed eyes. The parameter considered is the stability index (SI; percentage score where $100 \%$ is a theoretical task performed with maximum stability). Results: Since, no significant differences were found between limbs, the analysis was performed on the average results. The SI improved from $84.14 \% \pm 10.10$ to $86.96 \% \pm 5.60$ with opened eyes $(\mathrm{p}<0.05)$ and from $52.22 \% \pm 13.87$ to $56.25 \% \pm 17.60$ with closed eyes $(\mathrm{p}<0.05)$. Discussion: Study results suggest that a structured physical activity program improve balance ability with a consequence impact on the fitness and quality of life of osteoporotic people. In particular, including balance exercises in a training program is effective in improving postural control and proprioception. The latter seems to have greater benefit and is very important in poorly lighting places or conditions of sudden instability. (Figure 16)

\section{Applications: Work/Circadian Rhythm}

Changes in 24-Hour Movement Behavior During the Transition to Retirement: The Finnish Retirement and Aging Study (FIREA)

Anna Pulakka ${ }^{1}$, Saana Myllyntausta ${ }^{2}$, Tuija Leskinen ${ }^{3}$, Jaana Pentti ${ }^{3}$, Jussi Vahtera $^{3}$, Sari Stenholm ${ }^{3}$

${ }^{1}$ Finnish Institute for Health and Welfare, ${ }^{2}$ University of Eastern Finland, ${ }^{3}$ University of Turku

Introduction: Retirement changes time use and may therefore induce changes in 24-h movement behavior. This longitudinal study examined changes in daily time spent in sleep, sedentary time (SED), light physical activity (LPA) and moderate-to-vigorous physical activity (MVPA) before and after retirement by gender and occupation. Methods: The study population consisted of 569 participants (mean age 63.2 years, SD 1.1, $85 \%$ women) from the FIREA study. Daily time spent in sleep, SED, LPA and MVPA were measured with a wrist-worn triaxial ActiGraph accelerometer for one week before and after retirement with one-year interval. The accelerometer data were processed using the R package GGIR and threshold values of $<30 \mathrm{mg}$ for SED, $\geq 30 \mathrm{mg}$ for LPA and $\geq 100.6 \mathrm{mg}$ for MVPA were used. Sleep time was estimated based on combination of the GGIR sleep algorithm and self-reported in-bed and out-of-bed times. Occupational status was categorized as manual and non-manual. Linear mixed models were used to examine changes in 24-hour movement behavior in the transition to retirement. Compositional data analysis will also be conducted and results presented at the conference. Results: Women retiring from manual occupations reduced LPA by $52 \mathrm{~min}$ (95\% confidence interval [CI] 38-66) and MVPA by 11 min (95\% CI 7-15), whereas no marked changes in LPA or MVPA were observed among non-manual workers (Figure 17). In addition, SED increased by 28 min (95\% CI 12-44) among women retiring from manual occupations, but reduced by $23 \mathrm{~min}$ (95\% CI 11-35) among non-manual workers. Sleep increased among women retiring from both manual and non-manual occupations by 34-38 min. No marked changes in SED, LPA nor MVPA were observed among men, but sleep increased by 26 min (95\% CI 14-38) among non-manual workers. Discussion: The transition to retirement induced changes in 24-h movement behavior towards increased sleep and less physical activity among women, especially among those retiring from manual occupations.

Circadian Rest-Activity Rhythm and Falls in the Older Adults: A Prospective Cohort Study in Hong Kong

Zhihui $\mathrm{Lu}^{1}$, Timothy Chi Yui Kwok ${ }^{1}$

${ }^{1}$ The Chinese University of Hong Kong

Introduction: Given the rapid ageing population worldwide, falls have become a critical public health issue. There is evidence that dysregulated sleep were associated with increased risk of falls. Nonetheless, the association between circadian rest-activity rhythm and falls remains uncertain. Methods: Community-dwelling older adults $(82.7 \pm 3.8$ years) recruited from the MrOS and MsOS Hong Kong cohort were invited to wear the Actigraph accelerometer on wrist for seven days to measure circadian rhythm. Sleep complaints were assessed by questionnaire interview. The incidence of falls was ascertained by trimonthly telephone follow-up for one year. Nonparametric and cosinor analysis were used to calculate the variables describing the phase of circadian rhythm, including: onset time of the most active 10 hours (M10), and acrophase (time of peak activity). Based on cut-points derived from Classification and Regression Tree analysis, these circadian variables were then categorized into three groups as phase-advanced, reference and phaseddelayed. Logistic regression was used to investigate the association between the circadian rhythm and falls. Results: Ninety-one of 625 (14.6\%) participants experienced at least one fall within 12 months. Compared with the reference group, both early and later onset time of M10 were associated with a higher risk of falls. The association remained significant upon adjustment for other fall-related covariates, including sleep duration and complaints. Furthermore, later acrophase was also associated with increased risk of falls even with covariates adjusted. Discussion: Independent of fall-related risk factors including sleep duration and complaints, older adults with either early shifting or delay shifting in circadian phase may be at greater risk of falls. These findings imply that circadian rhythm disruption might be considered as a useful marker for falls, and circadian therapies might open up opportunities for fall prevention. (Figure 18)

\section{Hourly Cumulative Physical Activity Patterns in Community-Dwell- ing Middle-Aged and Older Adults}

Makoto AYABE ${ }^{1}$, Hideaki KUMAHARA ${ }^{2}$, Hideaki KUMAHARA ${ }^{3}$, Kensaku SASAYAMA ${ }^{4}$, Kazuhiro MORIMURA ${ }^{5}$, Takashi OYAMA ${ }^{1}$, Seiji SAITO $^{1}$, Yoshihide INUKAI ${ }^{1}$

${ }^{1}$ Okayama Prefectural University, ${ }^{2}$ Nakamura Gakuen University, ${ }^{3}$ Mie University, ${ }^{4}$ Mie University, ${ }^{5}$ Shujitsu University

Introduction: The present investigation aimed to examine the contribution of cumulative hourly physical activity (PA) patterns to the amount of 24-hour moderate-to-vigorous intensity PA (MVPA). Methods: Forty-two middleaged to older adults (mean age: $64.3 \pm 6.2$ years) were divided into inactive (IN), moderately active (MO), and active (AC) groups based on their 24-hour MVPA amounts. All participants wore a uniaxial accelerometer (Lifecorder, Kenz) on their waist for 7 consecutive days. The hourly PA was summed from 0:00 am to calculate the cumulative value, and the hourly cumulative value was divided by the 24-hour amount to calculate the relative cumulative value. Results: The MVPA category and time of day significantly affected the hourly PA for LPA, MVPA, and step count ( $p<0.05$ ). Cumulative LPA, MVPA, and step count differed significantly among the MVPA categories from early morning to midnight ( 6 am to $11 \mathrm{pm}, \mathrm{p}<0.05$ ). The amount of MVPA was significantly greater in the AC group than in the IN group at 6 am $(\mathrm{p}<0.05)$, and this difference was maintained until midnight. Discussion: The present study is the first to report that the contribution of PA in the morning hours represents $60 \%$ or more of the daily amount in the most active MVPA tertile. The difference observed among the MVPA tertile in the morning hours was maintained throughout the day. Hourly PA patterns differed based on the level of daily MVPA, and PA during the morning hours was identified as an important contributor to greater amounts of daily MVPA.

Is Rest-Activity Rhythm Long Term Associated With Mortality? The Como Vai Study

Andrea Wendt ${ }^{1}$, Luiza Ricardo ${ }^{1}$, Renata Bielemann ${ }^{1}$, Inácio CrochemoreSilva ${ }^{1}$

${ }^{1}$ Federal University of Pelotas 
Introduction: Circadian rhythm (CR) controls a series of physiological processes, including rest-activity rhythm (RAR), often measured by intraday variability (IV) and stability (IS). The first indicates rhythm fragmentation in a 24-hour cycle, while the second indicates similarity of the 24 hour-pattern across the days. This study aims at verifying the association between RAR and risk of all-cause mortality in older adults belonged to a population-based cohort. Methods: Como vai study is based on a population-based cohort comprising over 59-year-old individuals in Pelotas (Brazil). This analysis uses data from baseline (2014) and the first follow-up (2017), with RAR (IV and IS), measured by accelerometry (GeneActiv), as exposure, and deaths up to April 2017 as outcome. We present hazard rations (HR - Cox regression), considering sex, age, socioeconomic status, years of education, smoking, and morbidity score as covariates. Further adjustments for overall physical activity (OPA), moderate-to-vigorous physical activity (MVPA), inactivity, and total sleep time (TST) were applied. Results: From the 971 individuals included a total of 80 deaths were identified. Crude analysis showed that the increase of 1 standard deviation(SD) in IV, increased risk of death in 1.41 times $(1.19 ; 1.68)$, while the increase of $1 \mathrm{SD}$ in IS, decreased the risk in $47 \%$ $(\mathrm{HR}=0.57 ; 95 \% \mathrm{CI}: 0.45 ; 0.72)$. Associations remained even after sociodemographic adjustment. When adjusting for sleep and physical activity (PA), OPA and MVPA annulled the effect of IV and IS over mortality. Discussion: RAR seems to impact on mortality for older adults. These variables might reflect the $\mathrm{CR}$, responsible for many physiological processes, such as cytokines and hormonal secretion. Our adjusted analysis showed that PA annulled the effect of IV and IS in risk of death. PA may collaborate to CR regulation, and consequently, lower IV and higher IS. Interventions focused on PA for older people may help decreasing death risk.

\section{The Role of Age, Sex, and Pubertal Status in Patterns of Objectively Assessed Physical Activity and Sleep Patterns Among Youth}

Michelle Theodory ${ }^{1}$, Yao Xiao ${ }^{2}$, Andrew Leroux ${ }^{3}$, Vadim Zipunnikov ${ }^{4}$, Diana Paksarian ${ }^{1}$, Michael Milham ${ }^{2}$, Kathleen Merikangas ${ }^{1}$

${ }^{1}$ National Institute of Mental Health, ${ }^{2}$ Child Mind Institute, ${ }^{3}$ University of Colorado, ${ }^{4}$ Johns Hopkins Bloomberg School of Public Health

Introduction: A decline in physical activity (PA) across adolescence is associated with physical and mental health conditions, but the underlying biological and social correlates have been inconsistently identified. Moreover, research on adolescent PA often rely on subjective measures and focus on either PA or sleep (SL), rather than the full range of domains of PA, SL and circadian rhythms (CR). Here, we examine the associations between age, sex, and pubertal status with objectively assessed PA, SL and $\mathrm{CR}$ in a sample of youth in the New York City area. Methods: There were 328 male and 190 female participants aged 5-21 in the Healthy Brain Network Study (Alexander et al., 2017). Actigraphy measures were derived from the wrist-worn ActiGraph wGT3X-BT for participants with $95 \%$ wear time of at least 3 days. PA was analyzed as mean activity count (MAC), moderate-to-vigorous (MVPA) duration, and sedentary duration. SL was analyzed as SL duration, efficiency, and midpoint. CR was analyzed as timing of most active 10 hours (M10) and least active 5 hours (L5). Pubertal status (menarche for females and voice change for males) was self-assessed through the Peterson Puberty Scale. Results: Males had higher initial MAC than females and a larger decrease by age than females. MAC, MVPA, sleep duration, and M10 decreased with age and puberty. Sleep midpoint and L5 were later, however, for older youth. Sex differences were found among pubertal status' association with SL efficiency, sedentary duration, and some CR measures. Discussion: Our findings confirm previous research regarding a decline in PA and sleep duration, as well as a shift to later sleep midpoint across adolescence for both sexes.
This study concomitantly and uniquely elucidates changes in multiple measures of PA, sleep, and CR with age and puberty by sex - all of which have been linked to mental health. Future analyses will examine the influences of disorders and impairment as they are associated with PA and sleep.

\section{Technology \& Algorithm Development: Algorithm Dev}

Classifying Stepping Behaviour Using a Combined Cadence-Duration Heuristic

Craig Speirs ${ }^{1}$, Kate Lyden $^{2}$, David Loudon ${ }^{1}$, Malcolm Granat ${ }^{3}$

${ }^{1}$ PAL Technologies Ltd, ${ }^{2}$ KAL Research \& Consulting, LLC, ${ }^{3}$ University of Salford

Introduction: When analysing stepping behaviour, intensity (cadence steps per minute (spm)) or stepping volume thresholds are often used to classify bouts of stepping. This provides little context about the purpose or location of the stepping. A classification model that moves beyond volume based thresholds should allow these to be explored. In this study we propose a heuristic to classify bouts of stepping, using it to characterise daily volumes of stepping within a population of older adults. Methods: We identified a subset of 710 older adults from the NIH NCI iData study with at least 7 days (over 10 hours valid wear and 500 steps) of thigh-worn accelerometer data. We classified the stepping using cadence (thresholds at 75 and 120spm) and duration (thresholds at 60s and 15 minutes). Stepping bouts shorter than 10 s were classified as incidental stepping. For each subject we calculated mean daily step count for each class of stepping bout. These were then used to calculate mean daily step counts (and standard deviation) across the population. Results: In total 5081 days of activity were analysed. The mean daily step count was 7708 (3136), of which 1150 (418) were classified as incidental stepping. The distribution of stepping obtained from our heuristic is shown in Figure 19. As stepping bout duration increased, a larger proportion of stepping was undertaken at higher cadences. Discussion: We suggest this classification identifies three distinct groups of stepping behaviour: 1. Indoor stepping (short duration, low cadence) 2. Outdoor stepping (intermediate duration and cadence) 3 . Exercise related running (long duration, high cadence) There is also a substantial number of shorter duration, intermediate cadence steps we suggest are associated with longer bouts of outdoor stepping broken up by periods of standing. By grouping steps by upright bout we could extend the heuristic to associate this stepping with longer bouts of stepping characteristic of outdoor stepping.

\section{Comparing an Objective Measure of Foot Abduction Brace Non-Wear Time With Self-Reported Measures}

\section{Natan Silver ${ }^{1}$, Benjamin Griffiths ${ }^{2}$, Malcolm Granat ${ }^{2}$, Ehud Lebel ${ }^{3}$ \\ ${ }^{1}$ Shaare Zedek Medical Center, ${ }^{2}$ University of Salford, ${ }^{3}$ Shaare-Zedek} Medical Center

Introduction: The recommended treatment for idiopathic congenital clubfoot deformity is a non-operative bracing method called a foot abduction brace (FAB). Depending on the age of the child, the orthotic should be worn for predetermined time periods that reduce in duration as the child develops (Figure 20). Compliance is vital to achieve optimal functional outcomes and reduce the likelihood of deformity reoccurrence or surgery. However, compliance is typically monitored by self-report, which is timeconsuming to administer, provides only summary information, and lacks accuracy due to interpretation of the questions, social bias, and recall. This work presents a method for objectively monitoring FAB non-wear time using a single 3-axis accelerometer. Methods: Three participants mounted 
the device on their child's FAB for a range of days (3-7) during their normal treatment. Participants recorded wear and non-wear periods in a diary. 60-second epoched activity count data was calculated from raw $20 \mathrm{~Hz}$ data using open-access MATLAB functions. To objectively capture non-wear periods, a threshold-based open-access prosthesis non-wear algorithm was modified for FAB accelerometer count values. The methods were compared by calculating mean average percentage errors (MAPE) for each participant. Results: Both methods produced similar non-wear time duration with MAPE of $4.1 \%$. Furthermore, both methods produced similar non-wear patterns associated with the specific participant's treatment plan. Accelerometer raw data showed clear periods of FAB non-wear which were reported as wear, highlighting the need for objective compliance assessment. Discussion: A single accelerometer mounted to a FAB orthotic can be used to objectively assess non-wear-time. The method described could overcome the limitations of self-reported measures and improve treatment assessment. The use of open-access non-wear algorithms provides a useful method for future assessment of prosthetic and orthotic devices.

\section{Impact of ActiGraph Sampling Rate and Inter-Monitor Comparabil- ity on Measures of Physical Activity in Adults}

Karin Pfeiffer ${ }^{1}$, Kimberly Clevenger ${ }^{1}$, Jan Brond ${ }^{2}$, Daniel Arvidsson ${ }^{3}$, Kelly Mackintosh ${ }^{1}$, Melitta McNarry ${ }^{4}$, Alexander Montoye ${ }^{5}$

${ }^{1}$ Michigan State University, ${ }^{2}$ University of Southern Denmark, ${ }^{3}$ University of Gothenburg, ${ }^{4}$ Swansea University, ${ }^{5}$ Alma College

Introduction: Different versions of ActiGraph monitors are sometimes used within a given research study, but there is little information regarding inter-monitor comparability of newer models. Additionally, although sampling rate has been shown to influence accelerometer metrics, its influence on measures of free-living physical activity has not been directly studied. The purpose of this study was to examine differences in physical activity metrics due to inter-monitor variability and chosen sampling rate. Methods: Adults $(n=20)$ wore two ActiGraph wGT3X-BT monitors for one week (hip placement), with one accelerometer sampling at $30 \mathrm{~Hz}$ and the other at $100 \mathrm{~Hz}$, which was downsampled to $30 \mathrm{~Hz}$. Activity intensity was classified using vector magnitude (VM), Euclidean Norm Minus One (ENMO), and Mean Amplitude Deviation (MAD) cut-points. Equivalence testing was used to compare outcomes. Results: There was a lack of intermonitor equivalence for ENMO, time in sedentary/light- or moderateintensity activity according to ENMO cut-points, and time in moderateintensity activity according to MAD cut-points. Between sampling rates, differences existed for time in moderate-intensity activity according to VM, ENMO and MAD cut-points and time in sedentary/light-intensity activity according to ENMO cut-points. Bias ranged from 2.0 (MAD) to 15.9 (VM) mg for inter-monitor and 0.3 (ENMO) to 46.6 (VM) mg for sampling rate. Mean differences would equate to differences in moderateto-vigorous-intensity activity over a 10-h wear-day of 3.6 (MAD) to 10.8 (ENMO) min.day-1 for inter-monitor comparisons or $3.6(\mathrm{VM})$ to 5.4 (ENMO) min-day-1 for sampling rate. Discussion: Epoch-level intermonitor differences were larger than differences due to sampling rate, but both may impact outcomes such as time spent in each activity intensity. ENMO was the least comparable metric between monitors or sampling rates

\section{Is Behaviour Complexity Associated With Functional Ability Among Older Adults?}

Timo Rantalainen ${ }^{1}$, Erja Portegijs ${ }^{1}$, Taina Rantanen ${ }^{1}$, Lotta Palmberg ${ }^{1}$, Laura Karavirta ${ }^{1}$, Sebastien Chastin ${ }^{2}$

${ }^{1}$ University of Jyväskylä, ${ }^{2}$ Glasgow Caledonian University
Introduction: Information about mobility, and physical ability may be encoded in the complexity of daily pattern of physical behaviour. Therefore, physical behaviour complexity metrics could provide novel insight regarding the relationship between physical behaviour and health. The purpose of the present study was to examine the association between physical activity behaviour complexity, and mobility and functional capacity among community-dwelling older adults aged 75,80 , and 85 years-of-age. Methods: A total of 309 participants wore accelerometers concurrently on the thigh and the trunk for at least 3 consecutive days. Five activity states (lying, sitting, standing, walking, or vigorous activity) were defined in three different temporal grains ( $5 \mathrm{~s}, 1 \mathrm{~min}, 5 \mathrm{~min})$ and Lempel-Ziv complexity was evaluated. We assessed mobility with lifespace assessment and functional capacity with distance in preferred pace 6 min walk, and short physical performance battery. Results: Weak positive associations were observed between daily physical activity behaviour complexity and mobility and functional capacity at the finest temporal grains in both sexes (Spearman rho 0.19 to 0.27 ; $p<0.05$ ). No significant associations were observed in the coarsest temporal grain in either sex. Discussion: Lempel-Ziv estimates of physical activity behavior complexity with a fine temporal grain seems to provide a reasonable marker of physical behaviour complexity among community-dwelling older adults. The coarsest 5 min temporal grain may have smoothed out physiologically meaningful short activity bouts. Since complexity encodes information related to timing, intensity, and patterning of behaviour, physical behaviour complexity could be an informative indicator of future health.

\section{The Importance of Time of Day for Step Accumulation}

Craig Speirs ${ }^{1}$, Kate Lyden ${ }^{2}$, David Loudon ${ }^{1}$, Malcolm Granat ${ }^{3}$

${ }^{1}$ PAL Technologies Ltd, ${ }^{2}$ KAL Research \& Consulting, LLC, ${ }^{3}$ University of Salford

Introduction: We have previously shown that steps are accumulated in longer bouts on more active days, independent of habitual activity level. In non-clinical populations, vocational constraints on daily activity can limit opportunities to increase stepping. To better inform physical activity promotion interventions it is important to know when steps are accumulated. In this study we investigated the relationship between time of day and step volume, outlining a method to visualise and quantify step accumulation by time of day in a population of older adults. Methods: We identified a subset of days of thigh-worn accelerometer data (over 10 hours valid wear and 5000 steps) from the NIH NCI iData study. To better understand how the steps are accumulated, we visualised daily stepping chronologically centred around midday. This allowed us to compare steps accumulated before and after midday. We further segmented days into night (12am-6am), morning (6am-12pm), afternoon (12pm-6pm) and evening $(6 \mathrm{pm}-12 \mathrm{am})$ periods, classifying days based on when the largest proportion of stepping was accumulated. For each period, we calculated the number of days where the period contained the largest proportion of stepping and plotted daily step accumulation (Figure 21 ). The mean daily step count (and standard deviation) for each period was then calculated. Results: In total 7631 days of activity data were analysed. Across the population, most steps were accumulated in the afternoon (53\%), then the morning $(35 \%)$. There were a small number of days where most steps were accumulated during night-time $(1 \%)$, with these days having significantly more stepping. Discussion: For the selected days, we found a preference for physical activity to take place in the afternoon or morning. As this is not a representative dataset, additional datasets could be used to test if this observation can be generalised. This approach could be extended to see if this preference is sustained on days with a high step count. 
Towards the Quantification of Daily-Living Gait Quantity and Quality in Patients With Huntington's Disease: Preliminary Results Based on a Wrist-Worn Accelerometer

Karin Keren ${ }^{1}$, Monica Busse ${ }^{2}$, Nora Fritz ${ }^{3}$, Lisa Muratori ${ }^{4}$, Eran Gazit ${ }^{5}$, Inbar Hillel ${ }^{5}$, Micky Scheinowitz ${ }^{1}$, Tanya Gurevich ${ }^{5}$, Noit Inbar ${ }^{6}$, Nurit Omer $^{6}$, Jeffrey Hausdorff ${ }^{5}$, Lori Quinn ${ }^{7}$

${ }^{1}$ Tel Aviv University, ${ }^{2}$ Cardiff University, ${ }^{3}$ Wayne State University, ${ }^{4}$ Stony Brook University, ${ }^{5}$ Tel-Aviv Sourasky Medical Center, ${ }^{6} \mathrm{Tel}$ Aviv Medical Center, ${ }^{7}$ Columbia University

Introduction: Huntington's disease (HD) leads to altered gait patterns and reduced daily-living physical activity. Accurate measurement of dailyliving walking that takes into account involuntary movements (e.g. chorea) is needed. We aimed to develop and validate measures of daily-living step quality and quantify in patients with HD using an accelerometer worn on the wrist for 7 days. Methods: 29 HD participants and 21 healthy agematched controls completed clinic-based disease-specific assessments and a standardized laboratory-based circuit of functional activities, wearing triaxial accelerometers on the wrists, legs, and trunk. These data were used to develop gait detection algorithms. Subsequently, participants wore a triaxial accelerometer on their non-dominant wrist for 7 days. Gait features that reflect walking quantity (e.g., steps per day) and gait quality (e.g., regularity) were extracted and the \% of walking bouts with "irregular" movements was also quantified. Results: Measures of daily-living gait quantity were similar in the HD and healthy controls ( $p>0.05)$, although the HD patients tended to have fewer long walking bouts $(>30 \mathrm{sec})$ during daily-living $(\mathrm{p}=0.053)$. HD participants with higher clinician-rated upper body chorea had a greater $\%$ of walking bouts with irregular movements compared to those with low chorea scores $(\mathrm{p}=0.060)$ and healthy controls $(\mathrm{p}<0.001)$ (Figure 22A). Even after accounting for irregular movements, within-bout walking consistency was lower in the HD patients compared to controls $(\mathrm{p}<0.001)$, while across bout variability of these measures was higher $(\mathrm{p}<0.001)$. Measures of within bout consistency and variability were associated with disease-specific measures of motor function (134810 Figure 22B). Conclusions: These results suggest that a wrist-worn accelerometer can evaluate the quantity and quality of daily-living gait in people with HD while also estimating and accounting for the influence of irregular (choreic-like) movements.

\section{Technology \& Algorithm Development: Validation}

\section{6-Minute Walk Distance Using Inertial Sensors}

Vrutangkumar Shah ${ }^{1}$, Carolin Curtze ${ }^{2}$, Kristen Sowalsky ${ }^{3}$, Ishu Arpan ${ }^{1}$, Martina Mancini ${ }^{1}$, Patricia Carlson-Kuhta ${ }^{1}$, Mahmoud El-Gohary ${ }^{3}$, Fay Horak $^{1}$, James McNames ${ }^{4}$

${ }^{1}$ Oregon Health and Science University, ${ }^{2}$ University of Nebraska at Omaha, ${ }^{3}$ ERT- APDM Wearable Technologies, ${ }^{4}$ Portland State University

Introduction: The six-minute walk test (6MWT), with the distance walked as the primary outcome, has been used for monitoring endurance, disease state, and intervention effects. Wearable inertial sensors offer the advantage of collecting walk distance as well as gait characteristics during the 6MWT. This study aimed to validate our inertial sensor-based algorithm for the distance walked in the 6MWT. Methods: 24 subjects (20 with Multiple Sclerosis and 4 healthy controls) wore 2 inertial sensors (Opals by ERT-APDM Wearable Technologies) on both feet. Participants were instructed to walk as far as possible, back-and-forth along a 15-m, straight walkway (marked with small horizontal lines every $1 \mathrm{~m}$ to calculate the distance walked). The walking distance algorithm was previously developed on a dataset of 109 older men performing a $400-\mathrm{m}$ walk test along a $20-\mathrm{m}$ straight walkway (mean absolute error of $7.68 \mathrm{~m}$ ). Briefly, the 3-dimensional trajectory of each foot was estimated using zero-velocity updates, and the digital distance walked was calculated as the sum of changes in horizontal position between the stance phases of the gait cycles. The digital distance was validated against the clinical distance by calculating percentage mean absolute error. Results: The proposed algorithm showed a percentage mean absolute error of $4.17 \%$ with respect to the clinical distance, resulting in a slight overestimation of the clinical distance. The mean absolute distance error between the digital and clinical distance was $19.77 \mathrm{~m}(\mathrm{~min}=1.80 \mathrm{~m}, \max =64.24 \mathrm{~m})$ for $6 \mathrm{MWT}$ over an average clinical distance of $474.42 \mathrm{~m}$. Discussion: Inertial sensor-based algorithm showed a potential to estimate the distance walked during the 6MWT. Further work is needed to test the generalizability of the proposed algorithm with different walkways and administrators in a larger cohort, as well as determining test-retest reliability. (Figure 23)

\section{An Open-Source and Wearable System for Measuring 3D Human Motion in Real-Time}

Patrick Slade ${ }^{1}$, Ayman Habib ${ }^{1}$, Jennifer Hicks ${ }^{1}$, Scott Delp ${ }^{1}$

${ }^{1}$ Stanford University

Introduction: Analyzing human motion is essential for diagnosing movement disorders and rehabilitation interventions. Optical motion capture systems are the current standard for estimating kinematics but require expensive equipment in a predefined space. Wearable sensor systems can estimate kinematics in any environment, but are generally less accurate than optical motion capture. Here, we present OpenSenseRT, an opensource and wearable system that estimates upper and lower extremity kinematics in real time by using inertial measurement units and a portable microcontroller. Methods: The system uses off-the-shelf parts costing approximately $\$ 100$ USD plus $\$ 20$ for each tracked segment (Figure 24A). The open-source software and hardware are scalable, tracking up to 14 body segments with one sensor per segment. Kinematics are estimated in real-time using a musculoskeletal model and inverse kinematics solver, with the maximum computation frequency depending on the number of tracked segments (Figure 24C). Motion capture was used a ground truth reference for comparing kinematic estimates. Results: We compared the OpenSenseRT system to optical motion capture and found an average RMSE of 4.4 degrees across 5 lower-limb joint angles during three minutes of walking $(n=5)$ (Figure 24B) and an average RMSE of 5.6 degrees across 8 upper extremity joint angles during a Fugl-Meyer task $(n=5)$. The error increased at one degree per minute due to drift in the sensor measurements (Figure 24D). Discussion: OpenSenseRT is an opensource, low-cost, easy-to-assemble, and customizable system for computing real-time kinematics. We have openly shared all hardware and software to allow other researchers to use the system. This system will enable researchers to translate research relying on kinematics into real-world solutions. The accessibility of this technology could reduce the barriers to perform state-of-the-art motion analysis in low-income countries, improving health on a global scale.

Calibration and Cross-Validation of Cut-Points for Sedentary Time and Moderate-to-Vigorous Physical Activity From Hip, Non-Dominant and Dominant Wrist in Older Adults

Jairo Migueles ${ }^{1}$, Cristina Cadenas-Sanchez ${ }^{2}$, Juan M Alcantara ${ }^{3}$, Javier Leal-Martín $^{4}$, Asier Mañas ${ }^{4}$, Ignacio Ara ${ }^{4}$, Nancy Glynn ${ }^{5}$, Eric Shiroma ${ }^{6}$

${ }^{1}$ Linköping University, ${ }^{2}$ Public University of Navarra, ${ }^{3}$ University of Granada, ${ }^{4}$ Universidad de Castilla-La Mancha, ${ }^{5}$ University of Pittsburgh, ${ }^{6}$ National Institutes of Health

Introduction: Accelerometers' accuracy for sedentary time (ST) and moderate-to-vigorous physical activity (MVPA) classification depends on 
accelerometer placement, data processing, activities, and sample characteristics. As intensities differ by age, this study sought to determine intensity cut-points at various wear locations in >70-year-olds. Methods: Data from 59 older adults were used for calibration, and from 21 independent participants for cross-validation. Participants wore accelerometers on hip and wrists while performing activities and having energy expenditure measured with portable calorimetry. Euclidean Norm Minus One G (ENMO, mg) and counts/5s were derived from accelerometers' raw signal. ST and MVPA were defined as $\leq 1.5$ metabolic equivalents (METs) and $\geq 3$ METs $(1 \mathrm{MET}=2.8 \mathrm{ml} / \mathrm{kg} / \mathrm{min})$. Receiver operator characteristic (ROC) analyses showed fair-to-good accuracy (Area under curve [AUC]:0.62-0.89). Results: ST cut-points were: $7 \mathrm{mg}$ (cross-validation: sensitivity $=0.88$, specificity $=0.80$ ) and 1 count $/ 5 \mathrm{~s}$ (cross-validation: sensitivity $=0.91$, specificity $=0.96$ ) for hip; $18 \mathrm{mg}$ (cross-validation: sensitivity $=0.86$, specificity $=0.86$ ) and 102 counts $/ 5 \mathrm{~s}$ (cross-validation: sensitivity $=0.91$, specificity $=0.92$ ) for non-dominant wrist; and $22 \mathrm{mg}$ and 175 counts/5s (no cross-validated) for dominant wrist. MVPA cut-points were: $14 \mathrm{mg}$ (cross-validation: sensitivity $=0.70$, specificity $=0.99$ ) and 54 count $/ 5 \mathrm{~s}$ (cross-validation: sensitivity $=1.00$, specificity $=0.96$ ) for hip; $60 \mathrm{mg}$ (cross-validation: sensitivity $=0.83$, specificity $=0.99$ ) and 182 counts $/ 5 \mathrm{~s}$ (cross-validation: sensitivity $=1.00$, specificity $=0.89$ ) for non-dominant wrist; and $64 \mathrm{mg}$ and 268 counts $/ 5 \mathrm{~s}$ (no crossvalidated) for dominant wrist. Discussion: These cut-points can classify ST and MVPA in >70-year-olds, based on two acceleration metrics (i.e., ENMO [mg], and counts) and three body attachment sites (i.e., hip and wrists). The cut-points showed fair to good accuracy, and high sensitivity and specificity in an independent sample.

Calibration and Validation of Physical Activity Cut-Points for activPAL4: A Pilot Study

Kaja Kastelic ${ }^{1}$, Jure Zitnik ${ }^{2}$, Nejc Sarabon ${ }^{3}$

${ }^{1}$ University of Primorska, Andrej Marusic Institute, ${ }^{2}$ InnoRenew, CoE, ${ }^{3}$ University of Primorska, Faculty of Health Sciences

Introduction: Thigh-worn accelerometery is an emerging choice for 24-h movement behaviours assessment, yet methods to assess moderate-tovigorous physical activity (MVPA) needs further development. The aim of this pilot study was to establish activity cut-points for moderate (MPA) and vigorous physical activity (VPA) for activPAL4, and to test its validity in a free-living setting. Methods: Fourteen participants (34.6 \pm 11.5 years, 7 male) underwent a protocol of activities while wearing activPAL3 and activPAL4 simultaneously. A linear regression was used to establish cutpoints for activPAL4, based on the previously published cut-points for activPAL3. Testing its validity, we compared one-day free-living data from 60 participants $(36.6 \pm 8.3$ years, 28 male) who wore activPAL4 (thigh-worn) and ActiGraph wGT3X-BT (waist-worn) simultaneously. Results: Newly established vertical axis activPAL4 cut-points for MPA and VPA were 11,780-29,852 and $\geq 29,853$ counts/15s, respectively. Our sample spent on average $54.6 \pm 26.8$ and $32.6 \pm 20.5 \mathrm{~min} /$ day in MVPA when measured with ActiGraph and activPAL4, respectively. ActivPAL4 consistently counted less MPA, VPA and MVPA minutes (-13.2, -8.8 and $-22.0 \mathrm{~min} /$ day, respectively). The agreement observed were poor for MPA $(\mathrm{ICC}=0.42,95 \% \mathrm{CI} 0.09-0.65 ; \mathrm{p}<0.000)$ and VPA $(\mathrm{ICC}=0.42,95 \%$ CI $0.09-0.64 ; \mathrm{p}<0.000$ ), and moderate for MVPA (ICC $=0.50,95 \% \mathrm{CI}$ $-0.07-0.78 ; \mathrm{p}<0.000$ ). Bland-Altman plot (Figure 25) revealed wide limits of agreement. Discussion: The agreement between activPAL4 and ActiGraph was similar to that reported when comparing self-reported and accelerometry measured physical activity. This pilot study showed that activity counts method to obtain MPA and VPA from activPAL4 could be used in large-scale epidemiological studies, but has a limited value for the intervention studies with limited number of participants. More accurate and precise methods to assess MVPA using thigh-worn accelerometry need to be development in the future studies.

Can Machine Learning Activity Classification Models Developed in Children With CP be Used in Children With an Acquired Brain Injury?

Stewart Trost ${ }^{1}$, Matthew Ahmadi ${ }^{2}$, Margaret O’ $\mathrm{Neil}^{3}$, Emmah Baque $^{4}$

${ }^{1}$ Queensland University of Technology, ${ }^{2}$ University of Sydney, ${ }^{3}$ Columbia University Irving Medical Center, ${ }^{4}$ Griffith University

Introduction: Machine learning (ML) models have emerged as a more accurate and versatile alternative to threshold methods. However, little work has been undertaken to develop classification models for children with neurological impairments. Our group has developed and validated ML activity classification models for youth with cerebral palsy (CP). However, the extent to which CP models generalize to other patient groups with movement challenges has not been investigated. Therefore, this study evaluated the performance of a $\mathrm{CP}$ activity classification model in an independent sample of children with Acquired Brain Injury (ABI). Methods: 27 children with an ABI completed a series of PA trials while wearing an ActiGraph GT3X+ on the hip least affected by neurological impairment. PA trials consisted of: slow-paced walking (SPW, $20 \mathrm{~m} / \mathrm{min}$ ), moderatepaced walking (MPW, $40 \mathrm{~m} / \mathrm{min}$ ), fast-paced walking (FP, max effort), block stepping (BS), and an internet-based video game providing physical and cognitive training (VG). 52 features were inputted into a Random Forest (RF) classifier for recognition of sedentary behavior (SED), standing utilitarian movements (SUM), and walking (WALK). Results: Seated resting periods were correctly classified as SED (99.3\%). For MPW and FPW instances, $99.1 \%$ and $100 \%$ were correctly classified as WALK. For SPW instances, $24.8 \%$ were classified as WALK and $75.2 \%$ as SUM. For BS instances, $62.8 \%$ were classified as WALK and $37.2 \%$ as SUM. For VG instances, $62.7 \%$ were classified as SUM, $30.1 \%$ as SED, and $7.2 \%$ WALK. Discussion: A RF classifier trained in children with CP generalizes well to children with ABI, particularly for recognition of purposeful walking. ML activity classification algorithms can help clinicians monitor the PA levels of their patients more effectively. Improved device-based measures of PA also enable health researchers to better understand the health benefits of regular PA in children with ABI.

\section{Comparing Self-Report and Multiple Device-Based Measures of 24- Hour Time Use}

Elisabeth Winkler ${ }^{1}$, Abdullah Alzhrani ${ }^{1}$, Margaret Cook $^{1}$, Kelly Johnstone $^{1}$, Genevieve Healy ${ }^{1}$, Bronwyn Clark ${ }^{1}$

${ }^{1}$ The University of Queensland

Introduction: Low-burden, accurate options for measuring multiple time uses may help studies examining the health implications of how daily time is spent in various waking behaviours and asleep. This study compared measures of 24-hour time use that vary widely in researcher and participant burden. Methods: Staff and students $(n=61,36 \%$ female, Mean \pm Standard Devation $38 \pm 11$ years) recruited from the University of Queensland wore two activPALs (thigh and chest) 24 hours per day for 7 days while completing a daily sleep and wear log. Of these, 29 reported their usual weekday, Saturday and Sunday time uses: time in bed (TIB), lying awake, sitting, standing, and moving. Weekly average time uses were calculated for: two activPALs (traditional VANE algorithm) with upright position on the chest separating lying from sitting, plus log-identified TIB and nonwear (L-2xVANE); one activPAL (CREA algorithm), with either CREAestimated TIB (CREA) or the log (L-CREA); and, self-report (SR; weighted average). Correlations and mean differences were calculated for the time-use compositions (c[], with uses ordered as above and excluding 
non-wear), in R 3.6.3 (compositions package). Results: Correlations between the time-use compositions (centred log-ratio transformed) were limited: $r=0.18$ for SR, $r=0.25$ for CREA, and $r=0.61$ for L-CREA versus L-2xVANE. Variation against L-2xVANE was in all SR time uses and primarily in device TIB and lying versus sitting. Compositional means differed significantly $(\mathrm{p}<.05)$ for CREA c $[.22, .22, .35, .14, .07]$ and somewhat for SR $\mathrm{c}[.33, .07, .36, .10, .13](\mathrm{p}=.10)$ and L-CREA $\mathrm{c}[.32, .05, .39, .15, .08](\mathrm{p}=.29)$, relative to L-2xVANE $\mathrm{c}[.33, .09, .34$, $.16, .08]$. Discussion: Neither the least burden (SR) nor intermediate (CREA) methods were identical to the intensive method involving two activPAL devices and a log. The CREA algorithm coupled with the log came closest. The SR option showed promise, however, participants tended to underreport standing and overreport moving.

Comparison of Wrist-Worn Versus Hip-Worn Actigraph Sensors to Assess Real-Life Physical Activity in Adults: A Systematic Review

\author{
Ruopeng Sun ${ }^{1}$, Nolan Gall ${ }^{1}$, Matthew Smuck ${ }^{1}$ \\ ${ }^{1}$ Stanford University
}

Introduction: Over the past decade, wrist-worn accelerometers gained popularity in commercial and research use. Yet, no consensus exists for standardized wrist accelerometry data collection and processing, especially in comparison to the historically used hip-worn accelerometers. In this work, through a systematic review we aim to identify and analyze discrepancies between wrist-worn versus hip-worn Actigraph accelerometers in measuring adult physical activity. Methods: A systematic review was conducted on studies involving free-living data comparison between hip-worn and wrist-worn Actigraph accelerometers among adult users. We assessed the population, study protocols, data processing criteria (axis, epoch, wear time correction, etc.), and outcome measures (step count - SC, sedentary time -SED, moderate to vigorous physical activity -MVPA, etc.). SC discrepancy was analyzed using meta-analysis, whereas metaanalysis was not attempted for others due to heterogeneous data processing criteria amongst the studies. Results: We screened 201 studies with 16 studies qualifying for inclusion in the systematic review. Through metaanalysis, wrist-worn Actigraph overestimates SC by an average of 3490 steps/day ( $\sim 50 \%$ more) in comparison with hip-estimated SC. Regarding SED and MVPA estimation, the wrist sensor consistently overestimates MVPA time while underestimating SED time, with discrepancies ranging from a dozen minutes to several hours, as a result of different data processing protocols. Discussions: Our findings demonstrate wrist-worn Actigraph accelerometers consistently overestimate daily step count and MVPA while underestimating SED time in comparison to hip-worn monitors. These substantial discrepancies between wrist and hip sensors call attention to the need for a cautious approach to interpreting data from different wear locations. These results may also serve as a reference for data comparisons among studies using different sensor locations. (Figure 26)

\section{Computer Vision is a Reliable Method for Counting People on Side-} walks and Streets

Gregory Dominick ${ }^{1}$, Richard Suminski ${ }^{1}$, Matthew Saponaro ${ }^{2}$

${ }^{1}$ University of Delaware, ${ }^{2}$ AI Whoo

Introduction: Sidewalks/streets are settings where a large proportion of outdoor physical activity (PA) occurs. Current methods to assess PA in these settings have limitations that could be addressed with today's technology. This study developed and tested a new method utilizing a wearable video device (WVD) and computer vision (CV) to assess PAs performed on sidewalks/streets. Methods: Trained observers wearing WVDs recorded short (5-39 s) videos while stationary along sidewalk/ street segments in low, medium, and high walkable areas during morning, noon, and evening periods over the course of 1 year. Counts of people engaged in PA along the segments was determined from the videos manually by two investigators (criterion) and CV using multiple deep convolutional neural networks. Results: A total of 379 and 400 individuals were counted by $\mathrm{CV}$ and manually, respectively in 1743 videos ( $\mathrm{M}=16.4, \mathrm{SD}=7.2 \mathrm{~s} / \mathrm{video} ; 1-8$ people/video). The $\mathrm{CV}$ method was reliable for presence/absence of people (Kappa statistics $(\kappa)=.89$; $\mathrm{p}<0.001$ ) and counts of people in the videos (intraclass correlation coefficient $($ ICC $)=.898 ; \mathrm{p}<0.001)$. CV's performance in detecting the presence/absence of people in the videos compared to the criterion was as follows: $83.4 \%$ true negative (CV and criterion indicated 1452 videos were without people); $13.7 \%$ true positive (CV and criterion indicated 239 videos were with people); $1.2 \%$ false negative (CV indicated 21 videos were without people but were with people according to criterion); $1.7 \%$ false positive (CV indicated 30 videos were with people but were without people according to criterion). CV's performance remained high even when only videos with people in them according to criterion $(\mathrm{n}=260$ videos) were examined (91.9\% true positives; $8.1 \%$ false negatives). Discussion: Computer vision can be used to reliably extract data on people engaged in PA along sidewalks/streets. This approach enhances measurement accuracy while reducing the burden of analysis.

\section{Concurrent Validity of the SOFIT and iSOFIT in 7th and 8th Grade Students From Temuco, Chile}

Sebastian Miranda-Marquez ${ }^{1}$, Damian Chandia-Poblete ${ }^{2}$, Nicolas AguilarFarias $^{1}$

${ }^{1}$ Universidad de La Frontera, ${ }^{2}$ Queensland University of Technology

Introduction: Physical education is an important opportunity for children and adolescents to engage in regular physical activity (PA) and therefore obtain its wide benefits. Assessing the PA accrued in physical education lessons (PE) is key to better understand if these are realizing their potential contribution. The instrument System for Observing Fitness Instruction Time (SOFIT) allows the assessment of lessons by direct observation, and recently a new digital version has been launched (iSOFIT). However, its validity has not been evaluated nor compared to its original paper-based version. Thus, this study aimed to assess the concurrent validity of the iSOFIT in a sample of Chilean secondary students using the paper-based SOFIT as the reference method. Methods: Two independent observers assessed random physical education lessons of 7th and 8th grade of 10 schools in Temuco, either using the SOFIT or the iSOFIT. Both versions assess simultaneously the student's activity, lesson context and teacher behavior. The concurrent validity of iSOFIT was explored using Bland-Altman plots and measures of error. Results: Sixty-five lessons were assessed in 26 classes. Observed lesson times showed mean differences that ranged between $-2.2 \%$ and $5.2 \%$. The class context was the dimension with the smallest differences, while teacher behavior showed the largest differences between iSOFIT and SOFIT. Student activity and lesson context showed higher precision and lower variability, while the largest errors and highest variability were found when observing teacher behavior. Discussion: The findings showed that the iSOFIT had comparable performance with the SOFIT, providing researchers an additional option for assessing PE with digital tools.

\section{Convergent Validity of Actiwatch and activPAL for Assessing Time} in Bed

Paul Hibbing ${ }^{1}$, Jordan Carlson ${ }^{1}$, Stacey Simon ${ }^{2}$, Edward Melanson ${ }^{2}$, Seth Creasy $^{2}$

${ }^{1}$ Children's Mercy Kansas City, ${ }^{2}$ University of Colorado Denver, Anschutz Medical Campus 
Introduction: Wrist actigraphy is the standard method for assessing freeliving time in bed (TIB). A TIB detection algorithm called CREA was recently released for the thigh-worn activPAL (AP) monitor. Using this new method, we tested convergent validity of TIB estimates between AP and a standard wrist actigraphy method (Actiwatch, AW). Methods: 29 adults wore an AW and AP concurrently for up to 14 consecutive days. Both monitors estimated nightly start/end times for TIB, with allowance for interruptions (i.e., time out of bed). The AP times were determined by the CREA algorithm, and the AW times were determined using a validated manual inspection method. For each night, minute-by-minute data were compared from both monitors, beginning at the earlier of the two TIB start times, and ending at the later of the two TIB end times. Within this period, we examined minute-by-minute classification agreement for TIB (yes vs. no). Regression analysis was used to test agreement between devices for total TIB h/night, where perfect agreement would follow the line of identity (i.e., intercept of 0 and slope of 1). A mixed effects model was used to account for nesting of observations within individuals, and both variables were centered on the grand mean of the AP. Results: Total TIB estimates were higher from the AP $(7.5 \pm 1.1 \mathrm{~h} / \mathrm{night})$ than the AW $(6.3 \pm 1.5 \mathrm{~h} / \mathrm{night})$. The regression analysis showed an intercept of $0.17 \mathrm{~h} /$ night $(95 \% \mathrm{CI}:-0.46,0.13)$ and slope of $0.40 \mathrm{~h} / \mathrm{night}(95 \% \mathrm{CI}$ : $0.31,0.51)$. Standard error of the estimate was $1.55 \mathrm{~h} / \mathrm{night}$. At the epoch level, $5.8 \pm 1.8 \mathrm{~h} /$ night were classified as TIB by both monitors (see Figure 27). Discussion: The AP estimated significantly higher TIB duration than the AW. However, there was no criterion measure in the study (e.g., self-reported TIB), so it remains unknown which is more accurate. Future studies should address criterion validity and also compare AP and $\mathrm{AW}$ in greater detail, e.g., for detection of interruptions and naps.

Correcting the Errors: An Algorithm for Improving Heart Rate Assessment With Commodity PPG Hardware

Tom Nemeth ${ }^{1}$, Greg Dominick ${ }^{1}$, Kyle Winfree ${ }^{1}$

${ }^{1}$ Northern Arizona University

Introduction: Commodity hardware offer significant advantages over many clinical grade wearables. Given that they are designed for consumer use, they are excellent candidates for longitudinal data collection. However, this comes with a cost, in that such commodity devices often contain errors not present in clinical grade research wearables. Here, we offer a model for error correction in heart rate estimates provided from a Fitbit. Methods: Healthy young adults $(\mathrm{N}=23)$ wore both a Fitbit Charge HR2 and Polar H7 (with ActiGraph GT9X) concurrently, and continuously for one week. This resulted in roughly 500,000 minutes of concurrent wear time data, with heart rate averaged to minute level resolution. Linear models were used for estimating feature window size, which found a 19 minute rolling window to be an optimal size in providing historical context. From here, features were extracted using a gaussian weighted average across all Fitbit measures (steps, HR, METs, PA level, distance, calories) with additional features of slope and variance. Models were built using linear and support vector regression. Stepwise variable selection was conducted with the goal of minimizing BIC. Results: Prior to model correction, the mean error between the Fitbit and Polar HR monitors was $7.13 \mathrm{bpm}$. Of all minutes, $77.8 \%$ of the Fitbit HR assessments were within $10 \mathrm{bpm}$ of the Polar HR monitor. The linear model improved $(\mathrm{t}=30.9$, $\mathrm{p} \sim 0$ ) these metrics by reducing the mean error to $6.13 \mathrm{bpm}$ and $10 \mathrm{bpm}$ agreement to $83.5 \%$. Finally, the support vector regression results in a $5.15 \mathrm{bpm}$ mean error and overall agreement of $86.6 \%(\mathrm{t}=62.3, \mathrm{p} \sim 0)$. This is a $40 \%$ reduction in errors $(22.2 \%$ to $13.4 \%)$ compared to the original error between Fitbit and Polar. Discussion: Both models, the linear regression and support vector regression, provided a statistically significant improvement to the heart rate assessment of the Fitbit HR, using the
Fitbit measures as inputs to the model and Polar HR as the target output. (Figure 28)

Individual vs. Group Calibration of Machine Learning Models for Physical Activity Assessment Using Body-Worn Accelerometers

Alexander Montoye $^{1}$, Bradford Westgate ${ }^{1}$, Kimberly Clevenger ${ }^{2}$, Karin Pfeiffer $^{3}$, Joseph Vondrasek ${ }^{1}$, Morgan Fonley ${ }^{1}$, Joshua Bock ${ }^{4}$, Leonard Kaminsky $^{4}$

${ }^{1}$ Alma College, ${ }^{2}$ National Cancer Institute, ${ }^{3}$ Michigan State University, ${ }^{4}$ Ball State University

Introduction: Modeling approaches for translating accelerometer data into physical activity metrics are often developed using a group calibration approach. However, it is unknown if models developed for specific individuals will improve accuracy. Therefore, our study developed and compared accuracy of individually-calibrated vs. group-calibrated machine learning models for physical activity intensity assessment. Methods: Participants $(n=48)$ wore accelerometers on the right hip and nondominant wrist while performing activities of daily living in a semistructured laboratory and/or free-living setting. Criterion measures of physical activity intensity (sedentary, light, moderate, vigorous) were determined using direct observation. Data were reintegrated into 30second epochs, and eight random forest models were created to determine physical activity intensity by using all possible conditions of training data (individual vs. group), protocol (laboratory vs. free-living), and placement (hip vs. wrist). A 2x2x2 repeated-measures analysis of variance was used to compare epoch-level accuracy statistics (\% accuracy, kappa [k]) of the models when used to determine physical activity intensity in an independent sample of free-living participants. Results: Main effects were significant for the type of training data (group: accuracy $=80 \%, \mathrm{k}=0.59$; individual: accuracy $=74 \%[\mathrm{p}=0.02], \mathrm{k}=0.50[\mathrm{p}=0.01])$ and protocol (free-living: accuracy $=81 \%, \mathrm{k}=0.63$; laboratory: accuracy $=74 \%$ $[\mathrm{p}=0.04], \mathrm{k}=0.47[\mathrm{p}<0.01]$ ), but not for placement (hip: accuracy $=$ $79 \%, \mathrm{k}=0.58$; wrist: accuracy $=75 \%[\mathrm{p}=0.18] ; \mathrm{k}=0.52[\mathrm{p}=0.18])$. Point estimates for mean absolute error were generally lowest for the group training, free-living protocol, and hip placement. Discussion: Contrary to expectations, individually calibrated machine learning models yielded poorer accuracy than a group approach. Additionally, models should be developed in free-living settings to optimize predictive accuracy.

\section{Systematic Review of Device-Based Motion Sensors for Monitoring Physical Activity in Manual Wheelchair Users}

Kati Karinharju ${ }^{1}$, Kelly Clanchy ${ }^{2}$, Sjaan Gomersall ${ }^{1}$, Stewart Trost ${ }^{3}$, Sean Tweedy $^{1}$

${ }^{1}$ The University of Queensland, ${ }^{2}$ Griffith University, ${ }^{3}$ Queensland University of Technology

Introduction: Device-based motion sensors (DBMS), could potentially be applied to assess physical activities in manual wheelchair users (MWU). This study reviewed the evidence of the reliability and validity of the existing DBMS for estimating activities typical of daily wheelchair use. Methods: A systematic search was conducted in October 2019 and included studies were assessed using the COSMIN Methodology for Systematic Reviews of Patient-Reported Outcome Measures. Results: 29 articles evaluating 26 DBMS or sensor combinations met inclusion criteria (16 [62\%] accelerometer-based devices, 3 [12\%] combination of accelerometers and gyroscopes, 4 [15\%] inertial measurement units (IMU), 2 [7\%] gyroscope-based devices and 1 [4\%] power meter device). $16(62 \%)$ of the devices were custom-made and $10(38 \%)$ were commercially available, from which $9(90 \%)$ research-based devices and $1(10 \%)$ consumer-based device. 20 (77\%) devices from all DBMS provided an 
acceptable estimate of the validated outcome. Those based on 3-axial accelerometers and secured to the upper-arm or wrist provided the most accurate estimate of daily EE, self-propulsion and upper body exercising, whereas wheelchair mounted gyroscope and IMU devices provided the most accurate estimates of wheelchair movement time, distance and speed. The level of evidence based on the methodological quality and confidence level of truthfulness was 'very low' to 'low' for 17 (85\%) and 'moderate' to 'high' for $3(15 \%)$ devices. Discussion: Existing DBMS can provide indirect, but acceptable measure of habitual PA in MWU's. However, there is no single device that can provide a comprehensive and feasible measure of habitual PA for MWU's. Most approaches evaluated were validated in controlled settings with a combination of multiple devices and using elastic bands or straps when attaching the devices to the participants hand or wheelchair, which raises questions about their applicability for use in free living environment.

Technical Validation of the ActiGraph wGT3X-BT, GT9X, and Insight Watch Accelerometers

Joe Nguyen $^{1}$, Robert Brychta ${ }^{2}$, Kong Chen ${ }^{2}$

${ }^{1}$ ActiGraph, ${ }^{2}$ NIH/NIDDK

Introduction: ActiGraph accelerometers are used extensively to objectively assess physical activity, sedentary behavior, and sleep. With several generations of devices available for different research applications, an objective validation may help to characterize potential inter-generational differences in output arising from changes to hardware or firmware. Methods: A customized VWR orbital shaker was used to test the following ActiGraph devices (15 of each): wGT3X-BT (3X), GT9X (9X), and CentrePoint Insight Watch (CPIW). All devices were tested at two sampling frequencies, $30 / 32 \mathrm{~Hz}$ and $60 / 64 \mathrm{~Hz}$. A NIST-certified Lord Microstrain 3DM-GX5-25 (3DM) accelerometer was used as the criterion. The shaker was set to a radius of $5.08 \mathrm{~cm}$ and ramped from 0-250 RPM (0$3500 \mathrm{mg}$ ) in increments of $25 \mathrm{RPM}$. Raw vector magnitude (VM) was evaluated as a primary outcome. Results: The linearity of all devices was high ( $>99.9 \%$ ), there was no noticeable impact due to sampling frequency, and intermonitor variability for each device type was low $(\mathrm{CV}<1.0 \%)$. Baseline offset was (Mean $\pm \mathrm{SD})-11.2 \pm 7.3 \mathrm{mg}$ for the CPIW, $3.0 \pm 11.5 \mathrm{mg}$ for the $3 \mathrm{X}$, and $-21.8 \pm 25.3 \mathrm{mg}$ for the $9 \mathrm{X}$. Compared to the 3DM, the CPIW, 3X, and 9X averaged lower VM by 2.3 $(1754.1 \pm 5.9), 11.8(1744.6 \pm 9.4)$, and $31.0(1725.4 \pm 9.0) \mathrm{mg}$, respectively across all frequencies. VM was lower than the $3 \mathrm{DM}$ by $0.4 \% \pm 0.6 \%$ for the CPIW, $0.3 \% \pm 0.8 \%$ for the $3 \mathrm{X}$, and $1.8 \% \pm 0.5 \%$ for the $9 \mathrm{X}$ across all frequencies. As frequency increased above 50 RPM the relationship in percent difference from the 3DM was positive and linear for the CPIW (Figure $29 ;-1.0 \%$ to $0.4 \%)$, negative and linear for the $3 \mathrm{X}(0.4 \%$ to $-1.9 \%)$, and curvilinear for the $9 \mathrm{X}(-2.4 \%$ at $50 \mathrm{RPM},-1.2 \%$ at $150 \mathrm{RPM}$, $-2.1 \%$ at $250 \mathrm{RPM}$ ). Discussion: Using a shaker and criterion accelerometer we found small but consistent differences in acceleration across a wide frequency range between the ActiGraph devices evaluated. It is unknown how these differences translate to free-living.

Validation of a Multi-Sensor System to Detect Sedentary Screen Time in Overweight/Obese Adults

Alexander Tolas ${ }^{1}$, Rachel Lyons ${ }^{2}$, Kristina Hasanaj ${ }^{2}$, Amanda Tran ${ }^{1}$, Lemar Popal $^{1}$, Ajay Patel ${ }^{1}$, Matthew Buman ${ }^{2}$, Sarah Keadle $^{1}$

${ }^{1}$ California Polytechnic State University San Luis Obispo, ${ }^{2}$ Arizona State University

Introduction: Sedentary Screen Time (SST) consumes over half of leisure time for American adults, averaging $3.5 \mathrm{hrs} /$ day. Research states SST increases risk for chronic diseases and reduces longevity, however, most research is based on self-report questionnaires that ask about time spent watching TV. As the nature of how screen time consumption changes (e.g., streaming, social media) there is a need for technology-based systems that objectively assess SST. The purpose of this study was to validate a multi-sensor system to objectively detect SST in overweight or obese adults. Methods: Eight participants were enrolled (6 Female); (mean \pm SD) age $36.9 \pm 7.0 \mathrm{yr}$; BMI: $34.0 \pm 6.7 \mathrm{~kg} / \mathrm{m} 2$. Recreational SST was defined as leisure-time use of a screen for TV, movies, social media, or video games while sitting/lying. SST was assessed using the StandUPTV tablet application, which incorporates information from the Samsung tablet, TV using a WeMo Wi-Fi plug, and a Fitbit Charge 3. On two of the seven days, participants were asked to record two three-hour observation sessions using a GoPro camera on two random days across the seven day assessment period. Direct Observation (DO) was used as ground truth; all DO videos were manually coded by two trained researchers using Noldus Observer XT. Results: Based on DO, participants spent 1.44 hours (47.8\%) of the three-hour session in SST. The StandUPTV app estimated 1.77 hours of SST. On average, an overestimation of SST from the multi-sensor system of $0.33(0.13,0.54$ hours $)$ for the three-hour session was observed. From the StandUPTV app, 1.3 hours (73.7\%) was consumed through TV while 0.47 hours $(26.3 \%)$ through the tablet. Discussion: This study provides preliminary evidence that the multi-sensor system can objectively assess SST. Future research will examine whether estimates are improved by using a direct measure of posture (i.e., activPAL). The app is currently being refined for use in intervention studies designed to reduce SST.

\section{Validation of an Automated Sleep Detection Algorithm Using Multiple Accelerometer Data}

Tatiana Plekhanova ${ }^{1}$, Alex Rowlands ${ }^{1}$, Tom Yates ${ }^{1}$, Andrew Hall ${ }^{2}$, Melanie Davies ${ }^{1}$, Kamlesh Khunti ${ }^{1}$, Charlotte Edwardson ${ }^{1}$

${ }^{1}$ University of Leicester, ${ }^{2}$ University Hospitals of Leicester NHS Trust

Introduction: Commonly used accelerometer sleep detection algorithms rely on self-reported sleep onset and offset information. An algorithm to detect sleep in the absence of sleep diaries has been proposed, however, it has undergone limited validation. The aim of this study was to evaluate the validity of an automated sleep detection algorithm by assessing sleep estimates from three research-grade accelerometers worn on each wrist with concurrent laboratory-based polysomnography (PSG). Methods: Sixteen healthy volunteers (age $30.8 \pm 7.3$ years, BMI $25.5 \pm 3.6 \mathrm{~kg}$ / m2) wore an Axivity, GENEActiv and ActiGraph accelerometer on each wrist during one-night PSG assessment. Sleep estimates (sleep period time window (SPT-window), sleep duration, sleep onset and waking time, sleep efficiency, and wake after sleep onset (WASO)) were generated using the open-source GGIR package. Agreement of sleep estimates measured by accelerometers with PSG was determined using pairwise $95 \%$ equivalence tests $( \pm 10 \%$ equivalence zone), intra-class correlation coefficients (ICCs) with $95 \%$ confidence intervals and limits of agreement (LoA). Results: All accelerometer sleep estimates except for WASO were within the $10 \%$ equivalence zone of the PSG. Reliability between accelerometers worn on either wrist and PSG was moderate for SPT-window duration (ICCs $\geq 0.70$ ) and sleep duration (ICCs $\geq 0.70$ ), good for waking time (ICCs $\geq 0.78$ ), but poor for sleep onset (ICCs $\geq 0.44$ ), sleep efficiency (ICCs $\geq 0.41$ ) and WASO (ICCs $\geq 0.32$ ). The mean bias between all accelerometer sleep estimates worn on either wrist and PSG were low, however, wide 95\% LoA were observed for all sleep estimates, apart from waking time. Discussion: The automated sleep detection algorithm applied to data from Axivity, GENEActiv and ActiGraph accelerometers, worn on either wrist, provides comparable measures to PSG for SPT-window and sleep duration, sleep efficiency, sleep onset and waking time, but a poor measure of wake during the sleep period. 
Validity of the wGT3X+ for Activity Bout Analysis Using the Watershed Algorithm

Aline Taoum ${ }^{1}$, Quentin Delamare ${ }^{2}$, Pierre Jéhannin ${ }^{3}$, Guillaume Mahé ${ }^{3}$, Alexis Le Faucheur ${ }^{1}$

${ }^{1}$ University of Rennes, ${ }^{2}$ IRISA/Inria Rennes, ${ }^{3}$ University Hospital

Introduction: Among all the wearable monitors used in physical activity (PA) research, the ActivPAL provides an event-based classification from which stepping and non-stepping bouts are easily assessed. It would be interesting to propose such detection for the other well-known monitors deployed in PA research, such as the wGT3X+ accelerometer. We have previously developed and validated a methodology to detect walking activity during outdoor walking sessions only. Therefore, this study aimed to test the previously developed algorithm on wGT3X+ data in free-living measurements. Methods: 35 healthy older participants wore a wGT3X+ and an ActivPAL for a 7-day free-living period. The watershed algorithm was applied to wGT3X+ vector magnitude (VM) raw data and to both vertical axis (VA) and VM counts/s, with the low-frequency extension filter enabled (LFE) or not $(\mathrm{N})$. The threshold was computed using either the total 7-day data (Algorithm 1) or on moving windows of 1 hour (Algorithm 2). Then, the number of activity bouts and time of activity were compared with those obtained from the CREA algorithm of the ActivPAL software (PALanalysis v8.11.5.64) from the stepping and cycling activities combined. Results: The number of bouts/day and the total activity time/day were both significantly different when comparing all the analyzed data to the ActivPAL analysis $(\mathrm{P}<0.05)$. The activity time was overestimated by $>39 \%$ using the VM counts (LFE, N), and was underestimated by $<24 \%$ using the VA counts (LFE, N) for both algorithms (Figure 30). The raw data had the highest under- and overestimations of the activity time when using algorithms 1 and 2, respectively. Discussion: The watershed algorithm applied on wGT3X + VA counts/s (LFE) data showed promising findings (MAPE $\approx 11 \%$ ) in analyzing free-living activity bouts. Further work is required to improve the detection of free-living activity bouts using the wGT3X+ accelerometer.

Validity of Wrist- and Waist-Worn ActiGraph wGT3X-BT and Kenz Lifecorder for Step Counting At Walking and Running Speeds Under Controlled Conditions

Hideaki Kumahara ${ }^{1}$, Miwa Agune ${ }^{2}$, Yuuichi Watanabe ${ }^{2}$, Hibiki Aikawa ${ }^{2}$, Makoto Ayabe ${ }^{3}$

${ }^{1}$ Nakamura Gakuen University, ${ }^{2}$ Nakamura Gakuen University Graduate School, ${ }^{3}$ Okayama Prefectural University

Introduction: This study aimed to assess the validity of the ActiGraph wGT3X-BT (AG) and Kenz Lifecorder uniaxial accelerometer (LC) for step counting and compare the step outputs obtained from the wrist (AGwrist) and waist (AGwaist) ActiGraph attachment sites during walking and running. Methods: Twelve participants ( $23 \pm 7 \mathrm{yrs})$ concurrently wore the AGs at their waist and wrist whilst walking/running on a treadmill. Moreover, the LC is also worn on the waist. Treadmill speed was started at $40 \mathrm{~m} / \mathrm{min}$ and progressively increased by intervals of 15 or $20 \mathrm{~m} / \mathrm{min}$ (a 4 -min in each stage) until the participants exceeded over 17 (very hard) of the Borg RPE scale. Two observers visually counted the steps using a hand counter and subsequently confirmed the steps using the observations of video recordings to serve as the criterion standard. Results: The mean errors at all speeds were $-2.7 \pm 9.8 \%$, $-7.5 \pm 19.4 \%$ and $-46.4 \pm 14.6 \%$ for the LC, AGwaist and AGwrist, respectively. Significantly higher correlation coefficients for the criterion were observed in the steps determined using the waist-worn devices $(\mathrm{r}=0.98$ and 0.95$)$ as compared with the AGwrist $(r=0.63)$.
Moreover, the LC was significantly underestimated by $-31.2 \pm 18.7 \%$ only at the lowest walking speed. The AGwaist was significantly underestimated at 40 and $55 \mathrm{~m} / \mathrm{min}$ of walking speeds by $-68.0 \pm 15.3 \%$ and $-0.8 \pm 0.5 \%$, respectively. In contrast, the AGwrist was significantly underestimated by -50.9 to $-45.7 \%$ during $40-260 \mathrm{~m} /$ min speeds. Moreover, a significant but moderate relationship between the AGwaist and AGwrist was observed. Discussion: The AGwaist provided a valid estimate of steps taken during most walking and running, except at a very slow speed, and provides comparable results to the LC. However, a larger error was evident for the AGwrist, regardless of any walking/running speeds. Therefore, the step counts obtained from wrist- and waist-worn AG attachment sites are generally not comparable during both walking and running.

\section{Wrist-Worn Accelerometers Overestimate Arm Use in Stroke Patients When Not Correcting for the Effect of Walking}

Ruben Regterschot ${ }^{1}$, Ruud Selles ${ }^{1}$, Gerard Ribbers ${ }^{1}$, Hans Bussmann ${ }^{1}$

\section{${ }^{1}$ Erasmus MC}

Introduction: Studies increasingly apply wrist-worn accelerometers to measure arm use after stroke. Wrist-worn accelerometers measure arm use by recording all arm movements, including non-functional arm movement due to whole-body movements (e.g., walking, cycling). Recording non-functional arm movements as arm use may result in an arm use overestimation. It is unclear to what extent wrist-worn accelerometers overestimate arm use as a result of an effect of whole-body movements. Therefore, this study quantified to what extent wrist-worn accelerometers overestimate paretic arm use in stroke patients as a result of whole-body movements. Methods: In this study we assumed that all arm movements during whole-body movements are non-functional arm movements. We quantified the arm use overestimation by comparing the outcomes of two measurement methods: 1) arm use measured with wrist-worn accelerometers during all whole-body postures and movements (P\&M method), 2) arm use measured with wrist-worn accelerometers during sitting/standing only (sit/stand method). In a longitudinal observational cohort study we performed arm use measurements during weeks 3,12 , and 26 poststroke in 33 stroke patients. Results: At all time points, daily paretic arm use measured with the P\&M method was higher than measured with the sit/stand method $(\mathrm{p}<0.001)$. The mean difference in daily paretic arm use outcome between methods increased from $31 \%$ at week 3 to $41 \%$ at week 26 ( $\mathrm{p}<0.001)$. The differences in daily paretic arm use between methods were strongly associated with the daily walking time $(r=0.83-0.92)$. Changes in the daily paretic arm use difference between methods from week 3 to week 26 poststroke were strongly associated with the changes in daily walking time from week 3 to week 26 after stroke $(r=0.89)$. Discussion: This study shows that wrist-worn accelerometers overestimate paretic arm use in stroke patients when not correcting for the effect of walking.

\section{Technology \& Algorithm Development: eHealth}

The Feasibility of an Ecological Momentary Assessment to Measure Physical Activity and Sedentary Behaviour in Shift Workers

Malebogo Monnaatsie ${ }^{1}$, Stuart Biddle ${ }^{1}$, Adam Schmidt ${ }^{1}$, Amy Williams ${ }^{1}$, Anna Rogers ${ }^{1}$, Tracy Kolbe-Alexander ${ }^{1}$

${ }^{1}$ University of Southern Queensland

Introduction: Ecological momentary assessment (EMA) can be used to measure physical activity (PA) and sedentary behaviour (SB) in the realtime and natural environment. EMA might be suitable to address the atypical shift work schedules to understand the lifestyle behaviours. The 
aim of this research was to determine the feasibility of the Smartphone Ecological Momentary Assessment (SEMA) to measure PA and SB in shift workers. Methods: Full-time workers $(\mathrm{N}=128)$ received five SEMA prompts per day, for 7-10 days. The prompt survey included questions $(n=8)$ about PA and SB, location and time spent in the activity. SEMA prompts sent to shift workers were tailored according to work schedule, while prompts were sent at the same time of day for non-shift workers. One-Way ANOVA was used to assess outcomes in shift workers and nonshift workers. Results: Participants included 75 shift workers and 53 nonshift workers, $58 \%$ were female, and the mean age was $36(\mathrm{SD}=11)$ years. Compliance for completed prompt responses were similar between shift workers $(64 \%)$ and non-shift workers $(67 \%)(\mathrm{P}=.85)$. Missed prompts showed significant difference between the groups $(\mathrm{P}=.046)$. The findings related to prompt response showed that the most frequently answered SEMA was the first prompt of the day (24\%), while the 5th prompt received the least responses in both shift and non-shift workers. Self-reported physical activity was similar for shift and non-shift workers, but sedentary behaviour showed a significant difference between the groups. Discussion: Compliance for SEMA in our study is lower $(63 \%)$ compared to other studies using EMA in older adults (92\%) and adults (71\%). Therefore, to increase compliance in future, studies with shift workers including a post evaluation of the mobile EMA could be useful to understand usability. Keywords: shift work, ecological momentary assessment, physical activity, sedentary behaviour

Validity of Smartphone-Based Measurement of the Five Times Sit-toStand Test

Ronny Bergquist ${ }^{1}$, Francesca Gariboldi ${ }^{2}$, Ane $\emptyset_{\text {vreness }}{ }^{1}$, Emma Farina ${ }^{2}$, Kristin Taraldsen ${ }^{1}$, Beatrix Vereijken ${ }^{1}$, Jorunn Helbostad ${ }^{1}$, Sabato Mellone ${ }^{2}$

${ }^{1}$ Norwegian University of Science and Technology, ${ }^{2}$ University of Bologna

Introduction: Measuring physical function has been identified as a key factor for detecting early onset of functional decline in communitydwelling older adults. Instrumenting standardized clinical tests with inertial measurement units and smartphones can potentially enhance the sensitivity of clinical tests. Recently, an instrumented test battery has been developed in an app-format, designed for unsupervised self-testing in a home-setting. The test battery includes the Five Times Sit-to-Stand. The aim of this study was to evaluate the concurrent validity of the smartphone-based test using a camera system and force plates as gold standard. Methods: A convenience sample of community-dwelling older adults, 60 years and older, is being recruited in Trondheim, Norway. A smartphone (Samsung Galaxy S8) was fixed to the thigh in a position similar to when being worn in the trouser pocket. For the camera system (Vicon, $200 \mathrm{~Hz}$ ), the pelvis and right femoral coordinate systems were built according to. Pelvis and hip angles in the sagittal plane were calculated using Euler angles. The test ends when the body touches the chair; the seat contact was identified from a force platform (AMTI $1000 \mathrm{~Hz}$ ) under the chair. The initial and final stand/sit events were identified from the angular velocity of the pelvis in the sagittal plane according to previous studies. We used the Bland-Altman test to calculate the limits of agreement. ICC $(3,1)$ was used to assess interrater reliability. Results: Preliminary results reported in Figure 31 include a total of 30 trials from the first 17 participants. Discussion: The data collection and analyses are still ongoing. The measurement of the first sit-to-stand transition is the least accurate one but still with a maximum difference of $350 \mathrm{~ms}$. Preliminary results support the hypothesis that a pocket-worn smartphone can be a valid tool for selfadministering and instrumenting the five times sit-to-stand test.

\section{Technology \& Algorithm Development: User Centered Design}

\section{Monitoring Gait Developmental Trajectory in Preterm Children: A Sensor-Based Approach}

Maria Cristina Bisi ${ }^{1}$, Manuela Fabbri ${ }^{2}$, Duccio Maria Cordelli ${ }^{1}$, Rita Stagni ${ }^{1}$

${ }^{1}$ University of Bologna, ${ }^{2}$ IRCCS Institute of Neurological Sciences of Bologna

Introduction: Preterm children have an increased risk of motor delay. Gait analysis and wearable technology allows the assessment of motor performance in toddlers, identifying early deviations from typical development. Using a sensor-based approach, gait performance of preterm and full-term toddlers at different risk of motor delay was analysed. The aim was to measure quantitative differences among groups and provide a tool for early monitoring gait development. Methods: Three groups of 2 year old toddlers, matched for age and walking experience, participated in the study: preterm at high risk of motor delay (Hrisk-PT, $\mathrm{n}=8$, born at $<28$ gestational weeks or with $<1000 \mathrm{~g}$ of body weight), at moderate risk (Mrisk-PT, $\mathrm{n}=21$, born at 28-36 gestational weeks), and at low risk (FT, $\mathrm{n}=17$, born full-term). Children walked at self-selected speed wearing three inertial sensors on the lower back and on the shanks. Temporal parameters, short- and long- term variability, symmetry, and nonlinear metrics of trunk kinematics (i.e. harmonic ratio, recurrence quantification analysis, multiscale entropy) were calculated and statistically analysed with respect to risk of motor delay (Kruskal-Wallis test, significance level 5\%). Results: For increasing risk of motor delay (from FT to Hrisk-PT), children showed significantly longer stride-, stance- and double-supporttime, higher short-term variability and lower multiscale entropy values on the frontal plane (Figure 32). No difference was found for the other parameters. Discussion: Sensor-based gait analysis allowed differentiating gait performance of toddlers at different risk of motor delay, highlighting a less mature motor performance in those at higher risk (i.e. higher variability, lower complexity). The proposed set of parameters can serve as biomarkers for the early detection of the risk to develop persistent motor impairments.

\section{Multimodal Cues for Gait Rehabilitation With Smart Glasses in Persons With Parkinson's Disease (PD): A Methodology for the Selection of Effective Design Solutions}

Mattia Corzani ${ }^{1}$, Silvia Imbesi ${ }^{2}$, Giovanna Lopane ${ }^{3}$, Lorenzo Chiari ${ }^{4}$, Giuseppe Mincolelli ${ }^{2}$

${ }^{1}$ University of Bologna, ${ }^{2}$ University of Ferrara, ${ }^{3}$ IRCCS Istituto delle Scienze Neurologiche di Bologna, ${ }^{4}$ Health Sciences and Technologies Interdepartmental Center for Industrial Research (CIRI-SDV)

Introduction: Persons with PD are affected by motor impairments that compromise their autonomy. Recent advances in wearable systems showed that sensory biofeedback (BF) can improve gait in PD. Through verbal BF, an existing smartphone-based gait rehabilitation system (CuPiD-system) makes PD subjects better aware of their gait performance. Thanks to Smart Glasses (SG), real-time visual and haptic cues are also feasible: this study describes strategies to design an innovative mHealth system obtained by integrating the smartphone-based CuPiD-system with SG. The aim is to rehabilitate postural and transient gait disturbances and to provide gait training at home for persons with PD. Methods: We performed a pilot trial on five subjects not belonging to a particular category of users, following a Human-Centered design research approach. We proposed sensory BF suggesting a rhythm to be followed: auditory by wireless earphones, visual and haptic by the SG. We analyzed the subjects' qualitative and quantitative responses through an interview and a specific gait analysis protocol. Results: 
This testing phase investigated how sensory BF influences the user's gait, the most efficient cues to improve user's performance, and acceptance of the mHealth system. We applied an ad-hoc redesigned version of the QualityFunction-Deployment (QFD) design tool to manage the complexity of the collected data, Figure 33. Discussion: While visual BF improves spatial gait parameters, auditory and somatosensory BF improve temporal gait features (cadence). QFD's results confirm the role of sensory BF on gait rehabilitation: auditory and haptic BF reach a higher efficacy than the visual one. Some critical aspects emerged: the gap between the user's cadence and the target one; the subjects' sensory preferences. In the next phase on PD subjects, the target cadence will be subject-specific, and questionnaires should be used to evaluate subjects' sensory preferences and integrate them into the QFD matrix.

\section{Technology \& Algorithm Development: Future Perspectives}

Defining Continuous Walking Events in Free-Living Activities: Mind the Gap?

Abolanle Gbadamosi ${ }^{1}$, Benjamin Griffiths ${ }^{1}$, Alexandra Clarke-Cornwell ${ }^{1}$, Malcolm Granat ${ }^{1}$

${ }^{1}$ University of Salford

Introduction: Free-living activities range in duration, intensity, and are typically interspersed with interruptions of other behaviours; for example, standing events within walking bouts. Currently, there is no standardised method of characterising continuous walking. Processes that identify and describe interruptions that occur during walking are needed to better understand overall compliance to physical activity guidelines. This study seeks to develop a robust method of characterising continuous walking measured by an activity monitor. Methods: Participants $(n=24)$ were asked to wear the activPAL activity monitor for seven days to measure physical behaviours. A defined cadence threshold was specified as 100 steps/minute based on moderate-to-vigorous physical activity (MVPA) for a healthy population, and an algorithm was developed to re-define continuous walking events. The algorithm grouped walking events with short standing events as one single bout, provided the average cadence of the bout did not fall below the defined cadence threshold. Results: The average total time spent walking per day before applying the grouping algorithm was $123.1 \pm 36.6$ minutes and after grouping was $126.3 \pm 38.0$ minutes $(\mathrm{p}<0.001)$. The lower the defined cadence threshold, the higher the average time spent in MVPA (Figure 34). Furthermore, the composition of the grouped events comprised of short standing events and a few long walking events. Compliance to physical activity guidelines did not change as a result of grouping; however, there was an increase in the average time spent in MVPA ranging from 0.30 to 11.2 minutes after application of the grouping algorithm. Discussion: This study provides a robust and practical methodological approach to combining short interruptions in continuous walking by considering the intensity and duration of the activities included; therefore, providing a comprehensive evidence base to apply to physical activity guidelines and epidemiological studies.

Sensor-Based Ambulatory Assessment of Gross-Motor Development in School-Children: Influence of Age, Sex, and Anthropometry

Rita Stagni ${ }^{1}$, Alice Masini ${ }^{1}$, Stefania Toselli ${ }^{1}$, Sofia Marini ${ }^{1}$, Laura Bragonzoni $^{1}$, Andrea Ceciliani ${ }^{1}$, Marcello Lanari ${ }^{1}$, Alessandra Sansavini ${ }^{1}$, Alessia Tessari ${ }^{1}$, Davide Gori ${ }^{1}$, Laura Dallolio ${ }^{1}$, MariaCristina Bisi ${ }^{1}$

${ }^{1}$ University of Bologna

Introduction: Timely motor development in childhood is the foundation of healthy adult life. Several approaches have been proposed, but recently, wearable inertial sensors allowed its quantitative assessment in ambulatory conditions. The present study is comprised in the I-MOVE study and aims to analyze gross motor development in school children with respect to age, sex, and anthropometric characteristics. Methods: 150 children from primary school participated in the study, 72 first- (38M, 34F; age $75 \pm 5$ months) and 78 third grade $(48 \mathrm{M}, 34 \mathrm{~F}$; age $108 \pm 6$ months). Anthropometric characteristics (height, weight, triceps and subscapular skinfold thicknesses) were collected according to standardized procedures. Children walked at selfselected speed (NW) and in tandem (TW) wearing three inertial sensors on the lower back and on the shanks. Temporal parameters, short- and longterm variability, and nonlinear metrics of trunk kinematics (i.e. recurrence quantification analysis, multiscale entropy) were calculated and statistically analyzed (Kruskal-Wallis test 5\%). Results: Stance and double support duration increased during NW and decreased during TW with age independently from sex, while their variability decreased. Automaticity (i.e. recurrence indexes) increased with age during NW more in female than in males, while it decreased during TW independently from sex. Complexity (i.e. entropy) increased with age during TW. No dependency on height or weight alone was identified independently from age, while overweight (i.e. BMI and skinfold thicknesses) was associated to a reduction in the development of automaticity during TW independently from sex. Discussion: Both sex and anthropometry resulted to influence motor development in the target population, in particular overweight appears to delay the maturation of automaticity.

Timing of the Associations Between Objectively-Measured Physical Activity Levels and Glycemic Control and Variability Indices in the General Population: Results From the Food \& You Digital Cohort Study

\section{Douae El Fatouhi ${ }^{1}$, Harris Héritier ${ }^{2}$, Marcel Salathé ${ }^{2}$, Guy Fagherazzi ${ }^{3}$}

${ }^{1}$ Inserm U1018, Centre for Research in Epidemiology and Population Health, ${ }^{2}$ École Polytechnique Fédérale de Lausanne (EPFL), ${ }^{3}$ Luxembourg Institute of Health

Introduction: Disturbances of glycemic control and large glycemic variability in people without diabetes (DT) have been associated with increased risk of type 2 DT, cardiovascular diseases, and cancers. It is therefore important to identify lifestyle-related drivers such as physical activity to reduce glycemic variability and improve glycemic control among adults without DT. Methods: We analyzed data from 85 participants without DT of the Food \& You digital cohort. Physical activity, expressed as daily step count and assessed objectively using mHealth technologies (smartphone health apps or wearable activity trackers), was studied in relation to glycemic control and variability indices using generalized estimating equations models controlled for age, sex, BMI, smoking status, self-declared stress level, and for the daily step count measurement method. Glycemic indices were evaluated using data collected from 13 days of continuous glucose monitoring. Results: This study revealed no association between daily steps and glycemic indices of the same day. Our results suggest rather that every $1000 \mathrm{steps} /$ day increase in daily steps was associated with a $0.3588 \mathrm{mg} / \mathrm{dL}$, a $0.0917 \mathrm{mg} / \mathrm{dL}$, and a $0.0023 \%$ decrease in the maximum of glucose values, mean glucose, and in the glucose management indicator of the following day, respectively (95\% CI -0.6931 to $-0.0245 ; 95 \%$ CI -0.1793 to $-0.0042 ; 95 \%$ CI -0.0044 to -0.0002$)$. Discussion: Increasing the level of physical activity was linked to blunted glycemic excursions during the next day among individuals without DT. Because health-related benefits of a healthier lifestyle can be long to be observed, such potential short-term physiological benefits associated with certain behaviors could be integrated into health-related apps as personalized feedback to motivate individuals to engage in healthy behaviors. Our study gives also some insights into the 
promising possibilities offered by consumer wearable devices in epidemiological studies.

\section{Unsupervised Human Activity Recognition Using a Hidden Semi-} Markov Model on Wearable Sensor Data

Mariano Bernaldo ${ }^{1}$, Jack de Boer ${ }^{1}$, Claudine J.C. Lamoth ${ }^{1}$, Natasha Maurits $^{1}$

${ }^{1}$ University of Groningen, University Medical Center Groningen (RUG, UMCG)

Introduction: Monitoring of patients using wearables during their activities of daily living (ADL) could improve quality of care for patients with movement disorders. Human activity recognition (HAR) is a useful preliminary step to extract meaningful outcomes from the patient's movement. However, differences in disease characteristics, severity and patient characteristics may reduce the performance of solutions targeting the general patient population. Van Kuppevelt et al.(2019) recently explored the use of unsupervised machine learning as an alternative to a priori set threshold-based methods currently used in physical activity (PA) intensity assessment. We evaluate the possibility of using a similar method for HAR and explore alternative locations and number of sensors to improve accuracy. Methods: Nine healthy participants (mean 22.2, SD 1.7 years old), were measured during simulated ADL using Inertial Measurement Units (IMU) attached to the lower back, waist and sternum and both shanks, thighs, wrists and upper arms. A Hidden Semi Markov Model (HSMM) was trained to identify participant activity - sitting, lying, standing, walking - for every five second window using four features: the magnitude and the $3 \mathrm{D}$ angles of the acceleration vector. The results were compared to labels manually extracted from the measurement video. Results: When using the data from three accelerometers - sternum, left and right thigh - the trained model had a total accuracy of $71 \%$. The model could distinguish the basic postures and movements well, but performed worse on the recognition of 'intermediate' states, such as sitting comfortably on a couch (sitting-lying error) and slowly moving around the house (walking-standing error). Discussion: Preliminary results show that unsupervised learning may offer a viable HAR solution. Further research is necessary with different patient groups to test the reliability and robustness of the solution.

\section{Measurement \& Analysis: Big Data}

Compositional Data Analysis of Physical Behavior Among Finnish Adults in Relation to Cardio Respiratory Fitness

Henri Vähä-Ypyä ${ }^{1}$, Kari Tokola ${ }^{1}$, Pauliina Husu ${ }^{1}$, Harri Sievänen ${ }^{1}$, Tommi Vasankari ${ }^{1}$

${ }^{1}$ The UKK Institute for Health Promotion Research

Introduction: Studies measuring physical behavior (PB) on a $24 / 7$ basis and addressing the compositional nature of $\mathrm{PB}$ have gained increasing attention. This study investigated the PB patterns in relation to measured cardio respiratory fitness (CFR) using compositional analyses. Methods: The study was based on the FinFit 2017 study where the PB of 20- to 69year-old Finns was assessed 24/7 by triaxial accelerometer. During waking hours, the accelerometer was kept at the right hip, and during time in bed, at the non-dominant wrist. Light (LPA), moderate (MPA) and vigorous (VPA) physical activity were analysed with the mean amplitude deviation (MAD) method in $6 \mathrm{~s}$ epochs. The classification was done using both absolute 3.0 and 6.0 MET and relative individual $40 \%$ and $60 \%$ of the oxygen uptake reserve intensity thresholds. The angle for the posture estimation (APE) algorithm was used to identify lying, reclining, sitting and standing. Evaluation of time in bed was based on the wrist movement.
CRF was estimated by the 6 min walk test. Results: The composition of PB was entered into linear regression models via an isometric log-ratio transformation. Times spent in sleep $(\mathrm{p}<0.05)$ and reclining $(\mathrm{p}<0.05)$ were negatively associated with CRF, whereas sitting $(\mathrm{p}<0.05)$, standing $(\mathrm{p}<0.05)$, MPA $(\mathrm{p}<0.05)$ and VPA $(\mathrm{p}<0.05)$ were positively associated with CRF, when absolute thresholds were used. The relative thresholds inverted the physical activity results and only LPA time was positively associated $(\mathrm{p}<0.05)$ with CRF, whereas MPA $(\mathrm{p}<0.05)$ and VPA $(\mathrm{p}<0.05)$ were negatively associated with CRF. Discussion: Compositional data analyses with absolute and relative intensity thresholds provide novel and confusing insights into PB. Both the posture recognition and use of intensity thresholds based on individual fitness level facilitate interesting avenues for future investigations.

Differences in Accelerometer Measured Patterns of Physical Activity and Sleep/Rest Between Ethnic Groups Across Different Ages: An Analysis of UK Biobank

Nathan Dawkins ${ }^{1}$, Tom Yates ${ }^{1}$, Cameron Razieh ${ }^{1}$, Charlotte Edwardson ${ }^{1}$, Ben Maylor ${ }^{1}$, Francesco Zaccardi ${ }^{1}$, Kamlesh Khunti ${ }^{1}$, Alex Rowlands ${ }^{1}$

${ }^{1}$ University of Leicester

Introduction: Physical activity and quality sleep are important for health. Whether device-measured physical activity and quality of sleep differs by ethnicity and whether this varies by age-groups is unclear. Our aim was to compare physical activity and sleep/rest in white, South Asian and black ethnic group adults by age. Methods: Physical activity and sleep/rest disruption were assessed using 7-day wrist-worn accelerometer data for 87,917 participants, aged 45-79 years from UK Biobank. Data were stratified by sex and linear regression models used to analyse whether activity and sleep/rest disruption differed by ethnicity. An ethnicity x agegroup $(45-54,55-64,>65$ years) interaction term was used to assess whether differences between ethnicities were consistent across age-groups. Results: Compared to people of white ethnicity, overall physical activity was higher in people of black ethnicity, but lower in people of South Asian ethnicity. Nearly two-thirds (64\%) of black women and 70\% of black men accumulated 10 minutes of brisk walking per day, compared to $51 \%$ of white women, $54 \%$ of white men, $48 \%$ of south Asian women and $54 \%$ of South Asian men. Both minority ethnic groups had more disrupted sleep/ rest (poorer sleep quality) than white adults. Ethnicity $\mathrm{x}$ age-group interactions showed that the drop in physical activity and sleep quality by age-group category was delayed in both South Asian and black adults ( $>65$ years), relative to white adults ( $>55$ years) (Figure 35$)$. Discussion: While adults of black ethnicity are more active and South Asian adults less active than white adults, our data suggests that the age-related reduction in physical activity may be delayed in both South Asian and black adults. However, sleep disturbance is greater in South Asian and black adults, irrespective of age. Understanding how physical activity and rest differ by age and ethnicity may provide insight into chronic health conditions that are more prevalent in certain ethnic groups.

Muscle-Strengthening Activities Among Lesbian, Bisexual, and Heterosexual Women: National Health Interview Survey, 2013-2018

Andrea Kaniuka ${ }^{1}$, Cayla McAvoy ${ }^{1}$, Rajib Paul ${ }^{1}$, Catrine Tudor-Locke ${ }^{1}$

${ }^{1}$ University of North Carolina at Charlotte

Introduction: Muscle-strengthening activities (MSA) are associated with bone health, particularly for women. However, women are less likely than men to meet federal public health recommendations for MSA $\geq 2$ days/ week. Further, sexual orientation disparities for MSA may exist among women. Therefore, we examined the relationship between sexual orientation (i.e., lesbian, bisexual, other, and heterosexual) and engagement in 
MSA $\geq 2$ days/week among a nationally representative sample of adult women. Methods: Pooled Integrated Public Use Microdata Series-National Health Interview Survey (IPUMS-NHIS) data from 2013-2018 were restricted to women $\geq 18$ years of age. The sample $(\mathrm{N}=92,515)$ reported frequency of MSA engagement, sexual orientation, and demographic covariates (race/ethnicity, BMI, poverty, age, education, and marital status). Multiple survey logistic regression was conducted, regressing recoded bivariate engagement in MSA (yes/no meeting recommendations) on sexual orientation and listed covariates. Results: Under the adjusted regression, lesbian women were $39 \%$ more likely (AOR: 1.39, 95\% $\mathrm{CI}=1.20$ to 1.61 ) than heterosexual women to engage in $\mathrm{MSA} \geq 2$ days/week. Women with bisexual and other sexual orientations did not differ significantly from heterosexual women (bisexual: $\mathrm{AOR}=1.15$, $95 \% \mathrm{CI}=0.99$ to 1.34 ; other: $\mathrm{AOR}=0.89,95 \% \mathrm{CI}=0.63$ to 1.25 ). Minoritized race/ethnicity, BMI both above and below the normal range, poverty, increasing age, and having less than college education were associated with significantly lower odds of meeting MSA recommendations, and not being married was associated with significantly greater odds of meeting recommendations (Figure 36). Discussion: Women, in general, are failing to meet MSA recommendations and disparities across sexual orientations were identified. Research investigating barriers/facilitators to MSA, accounting for sexual orientation, is warranted to encourage participation in this healthful form of physical activity.

\section{Measurement \& Analysis: Machine Learning/Data Mining}

Assessing Gait in the Laboratory and in the Real World: The Impact of Environment and Bout Length on the Classification of Parkinson's Disease

Rana Zia UR Rehman ${ }^{1}$, Yu Guan ${ }^{1}$, Jian Shi $^{1}$, Lisa Alcock ${ }^{1}$, Alison Yarnall $^{1}$, Lynn Rochester ${ }^{1}$, Silvia Del Din ${ }^{1}$

${ }^{1}$ Newcastle University

Introduction: Parkinson's disease (PD) is a common neurodegenerative disease and gait impairment is typical. Applying machine learning (ML) models to real-world (RW) gait has potential to be more sensitive for PD classification as compared to laboratory (lab) data. Methodology and data aggregation levels (e.g. various walking bout length) for analysing RW gait may influence classification performance. The aim of this study was to investigate the impact of environment (lab vs RW) and bout lengths on ML models' performance for PD classification. Methods: 47 people with PD (68 \pm 9years) and 52 healthy controls (HC, age: $70 \pm 7$ years) were recruited from the ICICLE-GAIT study. In the lab, participants walked at their normal pace for 2 minutes; in the RW, participants were assessed continuously over 7 days. In both environments, 14 gait characteristics were quantified from a device attached on the lower back. RW gait characteristics were grouped by different bout lengths (e.g. bouts $<5$ seconds). Gait characteristic discriminative power (PD vs HC) was evaluated with the Area Under the Curve (AUC). ML models (support vector machine, random forest (RF), ensemble models) were trained on $70 \%$ and tested on $30 \%$ of the data. Results: RW gait characteristics from longer bouts (e.g. bouts $>60 \mathrm{~s}$ ) better discriminated PD vs HC compared to the lab $(0.51<\mathrm{AUC}<0.77)$, with RW walking speed showing the highest $\mathrm{AUC}=0.77$. Overall, $\mathrm{RF}$ trained on gait characteristics from bouts $>60$ seconds performed better $(\mathrm{F} 1 \mathrm{score}=77.20 \pm 5.51 \%)$ compared to the lab $(68.75 \pm 12.80 \%)$. Discussion: ML models applied to RW gait showed better classification performance compared to lab. Findings from this study suggest that choice of environment (i.e. RW) and data aggregation level (i.e. longer bouts) is important to achieve maximum discrimination performance (PD vs HC) as they have direct impact on ML performance for PD classification.
Classifying Lower-Limb Amputee Postures Using a Single ShankMounted Accelerometer

Benjamin Griffiths ${ }^{1}$, Laura Diment ${ }^{2}$, David Henson ${ }^{3}$, Malcolm Granat ${ }^{1}$

${ }^{1}$ University of Salford, ${ }^{2}$ University of Southampton, ${ }^{3}$ Imperial College London

Introduction: Current approaches for monitoring human physical behaviours (PB) by classifying postures use a single thigh-worn accelerometerbased device. In a lower-limb amputee population, we can embed the device within the prosthesis, eliminating issues with compliance and enabling increased battery size to extend monitoring time. However, embedding the device around the thigh would only allow us to monitor above-knee amputees, and, not below-knee amputees. Our work proposes a methodology for recognising PB postures (sitting, standing, and walking) from a single shank-located accelerometer-based device. We further suggest a novel technique for collecting labelled free-living PB data for developing machine learning (ML) algorithms to recognise $\mathrm{PB}$ postures. Methods: Free-living PB posture data (1 healthy, 1 amputee) was collected over 5 days using 2 devices. A thigh worn activPAL collected PB posture data as the ground truth, and an anterior-shank worn accelerometer collected 3-axis acceleration data at a rate of $20 \mathrm{~Hz}$. PB postures and the corresponding shank accelerometer data were extracted in 15 -second epochs, with a 5 second overlapping window, and a set of 136 features were calculated from each epoch's acceleration data. Linear discriminant analysis reduced the dimensions of the feature set down to 2 principle components which were given to a k-nearest neighbour algorithm $(\mathrm{k}=20)$ that used a Euclidean distance metric. Results were analysed using a leaveone-group-out cross-validation for both participants Results: Cross-validation provided a mean test accuracy (f1 score) of $84 \%$ across the 2 groups. Figure 37 shows the classification confusion matrix. Discussion: It is possible to accurately classify PB postures using a single shank located accelerometer and a 'shallow' ML model. Furthermore, this method enables long-duration studies on the PB patterns of lower-limb amputees within free-living.

\section{Detecting Freezing of Gait Using Raw Inertial Sensor Data From People With Parkinson's Disease}

Johanna O’Day ${ }^{1}$, Marissa Lee ${ }^{1}$, Kirsten Seagers ${ }^{1}$, Shannon Hoffman ${ }^{1}$, Scott Delp ${ }^{1}$, Helen Bronte-Stewart ${ }^{1}$

${ }^{1}$ Stanford University

Introduction: Wearable inertial measurement units (IMUs) offer potential for continuous monitoring of Parkinson's disease (PD). IMU-based algorithms that detect walking impairments like freezing of gait (FOG) are reliant on hand-engineered features that may be specific to certain freezing presentations, preventing generalizability. We aim to develop a model that uses raw IMU data to detect FOG. Methods: Seven participants with PD walked in a freeze-eliciting course wearing 6 IMUs on the chest, lower back (L5), shanks, and feet. Two experienced raters labeled FOG using video. IMU data were split into 2-s windows and classified by majority label (FOG or non-FOG). Each example was augmented with event timing perturbations and sensor placement rotations. We trained a 2-layer convolutional neural network with all participants' data to create a general model and with a single participant's data to create a participant-specific model. We trained the participant-specific model using the worst-performing participant from the general model. Models were evaluated using area under the receiver operator characteristic (AUROC) and average precision. Results: The general model could detect FOG from raw IMU signals $($ AUROC $=0.81)$. For the worst-performing participant $(\mathrm{P} 3$, AUROC $=$ 0.66 ), a participant-specific model improved FOG detection (AUROC $=0.85$ ) (See Figure 38). Average precisions for the general and 
participant-specific models were two-fold greater than baseline, 0.62 (baseline $=0.26$ ) and 0.70 (baseline $=0.28$ ). Discussion: General and participant-specific models trained with raw IMU data detect FOG with accuracies comparable to models that use hand-engineered features (AUROC $=0.77-0.96)$. This work demonstrates that model performance does not decline when using raw IMU signals, which may capture a wider set of FOG behaviors than hand-engineered features, thus indicating our models may better generalize across the PD population.

\section{Development and Evaluation of Wearable Devices-Based Physical Activity Intensity Classification Models in Manual Wheelchair Users With Spinal Cord Injury}

Zijian Huang ${ }^{1}$, Yousif Shwetar ${ }^{1}$, Akhila Veerubhotla ${ }^{1}$, Steven Knezevic ${ }^{2}$, EunKyoung Hong ${ }^{2}$, Ann Spungen ${ }^{2}$, Dan Ding ${ }^{1}$

${ }^{1}$ VA Pittsburgh Healthcare System, ${ }^{2}$ James J. Peters VA Medical Center

Introduction: There is a lack of convenient and accurate physical activity (PA) intensity self-monitoring methods for manual wheelchair users (MWUs) with spinal cord injury (SCI). Wearable devices have been widely used to monitor PA for their popularization and price reduction. We evaluated the performance of three published ActiGraph's proprietary "vector magnitude count(VMC)"-based PA intensity classification models for MWUs on an out-of-sample dataset. Raw accelerometer signals-based models were also developed and evaluated. Methods: Wrist-worn accelerometer data were collected from $60 \mathrm{MWUs}$ with SCI when performing a set of structured activities using ActiGraph wearable devices. The mean absolute deviation (MAD) and the euclidean norm minus one (ENMO) were derived from raw-accelerometer signals to develop cut-off threshold models, while random forest (RF) was used to develop the machine learning model. The VMC-based models were evaluated on all data that meet the inclusion and exclusion criteria, while the raw accelerometer signals-based models were evaluated with the train-test split technique. The model performance was characterized using recall, precision, specificity, and normalized Mathew's correlation coefficient (nMCC). Results: All models achieved good performance in distinguishing sedentary vs. non-sedentary activities with the nMCC ranged from 0.87 to 0.89 . However, all models showed limited performance in distinguishing moderate to vigorous PA (MVPA) vs. non-MVPA with the nMCC ranged from 0.76 to 0.79 . Discussion: Although the RF model marginally outperformed the MAD model and achieved the best performance, the MAD model is still more practical when considering the model complexity. While the MAD model may be used for tracking daily sedentary activities, more work needs to be done in the future to develop an accurate model for tracking MVPA.

\section{Physical Activity Levels of Patients With Chronic Low Back Pain and Central Sensitization: Insights From a Machine Learning Method}

Xiaoping Zheng ${ }^{1}$, Michiel Reneman ${ }^{1}$, Egbert Otten ${ }^{1}$, Claudine Lamoth ${ }^{1}$

${ }^{1}$ University of Groningen, University Medical Center Groninige

Introduction: Chronic low back pain (CLBP) is a leading cause of high levels of disability. Central sensitization (CS) is often present in CLBP. It is reported that physical activity (PA) can reduce the risk of disability, and is often recommended in management of CLBP. However, evidence of the relation between PA levels and CLBP is inconsistent and even less is known about association with CS. This study aimed to investigate PA patterns in patients with CLBP and low or high CS using an unsupervised machine learning method. Methods: Based on Central Sensitization Inventory, 42 patients were divided into: low (23; CLBP-) and high CS (19; CLBP). An accelerometer sensor was attached at right hip. For each patient, 4 days of data were used for analyses. From x, y, $\mathrm{z}$ axes, the vector magnitude was calculated. For each group, a Hidden semi Markov Model (HSMM) was trained. To determine the number of hidden states (PA patterns), Bayesian Information Criterion (BIC) was used. Differences in state duration time, state occupation time in day, state transitions were assessed with t-tests, and state composition with Jensen-Shannon divergence (JSD). Results: Indicated by BIC, 5 states HSMMs were selected. These states were: long-time sedentary (e.g.,sleeping), sedentary (e.g.,desk work), light activity (e.g.,vacuuming), light locomotion (e.g.,slow walking), moderate-vigorous activity (e.g.,running). JSD showed no difference between 2 HSMMs' state compositions. Differences in overall PA levels were non-significant $(\mathrm{p}>0.05)$. Differences in state transitions showed that between light and moderate activity, CLBP- more frequently have sedentary state. Discussion: This study observed that CLBP- and CLBP have no differences in overall PA levels, but have differences in the distribution of PA patterns over the course of performing light and moderate activities. CLBP- rested more frequently between light and moderate activities while CLBP maintained light and moderate activities longer. (Figure 39)

\section{Quantifying Activity to Study Healthy Ageing}

Stylianos Paraschiakos ${ }^{1}$, Arno Knobbe ${ }^{2}$, Eline Slagboom ${ }^{1}$, Marian Beekman $^{1}$

\section{${ }^{1}$ Leiden University Medical Center, ${ }^{2}$ Leiden University}

Introduction: While, nowadays, people generally live longer, they are increasingly facing health issues, leading to a reduction in functionality and quality of life along with an increase in medical costs. Sedentary lifestyles among the elderly mount to $10 \mathrm{hrs}$ inactivity per day. Hence combined caloric intake/physical activity interventions are applied to encourage the elderly to follow a more active and healthy lifestyle. These need to be carefully monitored. Methods: The Growing Old TOgether (GOTO) study introduces such an intervention, where a 13-weeks lifestyle program was applied, with a target of $12.5 \%$ caloric restriction and $12.5 \%$ increase in energy expenditure through an increase in physical activity, in 164 older adults (mean age $=63.2$ years; $\mathrm{BMI}=23-35 \mathrm{~kg}$ ) $\mathrm{m} 2$ ). Quantifying the changes in physical behaviour is of utmost importance in order to investigate their effect on health gains. Using data from wrist and ankle activity accelerometers (wearables) and machine learning models, we were able to recognize the different activities performed and to report their frequencies and durations both in baseline and after the 13weeks intervention. To quantify these observations, we built activity profiles that we intend to associate with changes in physiological parameters of health. Results: Our initial analysis of ankle and wrist acceleration magnitudes shows that on average, the post-intervention ankle magnitude was significantly $(\mathrm{p}=0.01)$ increased by $15 \%$ during day time (8-20:59 hrs). In detail, we observed that the main increase in activity levels for the female participants was late in the morning (10 13:00) while for the male ones late in the afternoon (14:00-16:00). On the other hand, the wrist magnitude did not have any significant difference. Discussion: As a result of the above, the baseline weight decreased by $4.2 \%$, while many other parameters of metabolic health, such as blood pressure, and thyroid, glucose and lipid metabolism improved significantly. (Figure 40)

\section{Scalar on Time-By-Distribution Regression and its Application to Modelling Cognitive Function in Alzheimer's Disease}

Rahul Ghosal ${ }^{1}$

${ }^{1}$ Johns Hopkins Bloomberg School of Public Health

Introduction: Wearable data is a rich source of information that can provide deeper understanding of links between human behaviours and human health. Existing modelling approaches use wearable data summarized at 
subject level via scalar summaries using regression techniques, temporal (time-of-day) curves using functional data analysis (FDA), and distributions using distributional data analysis (DDA). We propose to capture the interaction between temporal and distributional information in wearable data using subject-specific time-by-distribution (TD) data objects. Methods: we propose scalar on time-by-distribution regression (SOTDR) to model associations between scalar response of interest such as health outcomes or disease status and TD predictors. We show that two-way TD data objects can be parsimoniously represented via a collection of timevarying L-moments that capture distributional changes over the time-ofday. Results: The proposed method is applied to the accelerometry study of mild Alzheimer's disease (AD). Mild AD is found to be significantly associated with reduced maximal level of physical activity, particularly during morning hours. Discussion: It is also demonstrated that TD predictors attain much stronger associations with clinical cognitive scales of attention, visual memory, and executive function when compared to predictors summarized via scalar total activity counts, temporal functional curves, and quantile functions.

\section{Sedentary Time and Light Activity Features Differentiate People With Low Back Pain From Healthy Controls}

Ruopeng Sun ${ }^{1}$, Christy Tomkins-Lane ${ }^{2}$, Amir Muaremi $^{3}$, Patricia Zheng ${ }^{4}$, Manoj Mohan ${ }^{1}$, Matthew Smuck ${ }^{1}$

${ }^{1}$ Stanford University, ${ }^{2}$ Mount Royal University, ${ }^{3}$ Novartis Institutes for BioMedical Research, ${ }^{4}$ University of California San Francisco

Introduction: Physical inactivity is described as both a cause and a consequence of low back pain (LBP) largely based on self-reported measures. A better understanding of the connections between routine physical activity and LBP may improve LBP interventions. In this study, we aim to characterize the physical activity of people with LBP in comparison to healthy controls using accelerometers, and to derive a set of LBP-specific minute thresholds that may be used as targets for future physical activity interventions. Methods: Twenty-two people with LBP $(47.8 \pm 13.3 \mathrm{yrs}$ old $)$ were compared to 155 age and gender-matched healthy controls (from NHANES, $47.9 \pm 14.3 \mathrm{yrs}$ old). All subjects wore an ActiGraph accelerometer on the right hip for 7-consecutive days. Physical activity features were derived using the Physical Performance intervals recently introduced for populations with musculoskeletal pain, and Freedson's intervals. A random forest classifier was trained with leaveone-out cross-validation. An interpretation algorithm (SHAP) was applied to assess feature importance and to establish LBP-specific activity minute thresholds. Results: The classifier identified a set of 8 features (all related to sedentary and light activity, <800 CPM) that achieved $88.1 \%$ accuracy for distinguishing LBP from controls. Moderate-to-vigorous physical activity features were not found to be discriminative. In addition, we identified a set of daily time and intensity activity thresholds that are associated with the LBP prediction. Discussion: This study identified sedentary to light intensity activity, not moderate-to-vigorous, as key to discriminating people with LBP from healthy controls. Future research should investigate these features for response to change in LBP status and their utility in LBP patient monitoring. The time and intensity thresholds identified through this work may be explored as targets for future physical activity interventions aimed at LBP treatment or prevention (Figure 41)

\section{The Contribution of Gyroscope Data to Accelerometer Estimates of Free-living Physical Behavior Intensity}

Robert Marcotte ${ }^{1}$, Christos Pedone ${ }^{1}$, Patty Freedson ${ }^{1}$, John Staudenmayer $^{1}$, John Sirard ${ }^{1}$

${ }^{1}$ University of Massachusetts Amherst
Introduction: To compare free-living activity intensity classifiers trained using hip-worn accelerometer (ACC), gyroscope (GYRO), and combined $($ ACC + GYRO) data. Methods: Ten participants (age:31.1 \pm 16.0 years, $50 \%$ male) were video-recorded during one 4-hour session while wearing an ActiGraph GT9X (AG) on the right hip. Direct observation videos were annotated retrospectively for postural orientation, activity type, and activity intensity, which provided the criterion measure. Time- and frequencydomain AG signal features were computed within 15-second epochs from the raw accelerometer- and IMU gyroscope-sensor data. Separate random forest models were trained using ACC, GYRO, or both (ACC + GYRO) to estimate activity intensity category (sedentary, light, moderate-tovigorous) and evaluated using leave-one subject out cross-validation. Results: Balanced classification accuracy using ACC, GYRO, and ACC + GYRO data was $79.9 \% \pm 5.9 \%$ (mean $\pm \mathrm{SD}$ ), $81.5 \% \pm 5.0 \%$, and $83.8 \% \pm 4.7 \%$, respectively. Mean absolute percent error for estimated time spent in SED, LPA, and MVPA was lower using ACC + GYRO $(5.4 \% \pm 3.4 \%, 13.7 \% \pm 8.6 \%$, and $30.2 \% \pm 32 \%$, respectively) compared to using ACC $(7.6 \% \pm 3.6 \%, 24 \% \pm 28 \%$, and $34.2 \% \pm 32.3 \%$, respectively) or GYRO $(7.5 \% \pm 3.1 \%, 28.1 \% \pm 26 \%, 37 \% \pm 30 \%$, respectively). Limits of agreement were narrower for ACC + GYRO-estimated time spent in SED and LPA compared to using either ACC or GYRO alone (Figure 42). Discussion: ACC and GYRO sensors measure different aspects of human movement that may improve individual-level precision when used together compared to using either sensor independently. Further work should investigate the utility of multi-sensor devices for estimating aspects of physical behavior in a larger sample over extended periods of free-living behaviors.

\section{The Development of an Open Source Algorithm for Digital Biomar- kers of Step Cadence From Wrist-Worn Accelerometer Data Using the V3 Approach}

\section{Joshua Twaites ${ }^{1}$, Melvyn Hillsdon ${ }^{1}$, Joss Langford}

${ }^{1}$ University of Exeter

Introduction: Wrist-worn accelerometers continuously monitor accelerations associated with physical activity behaviours. In order to translate the acceleration data into measures of physical activity behaviours, algorithms are required. This work aims to develop digital biomarkers of step cadence, including daily step counts and time spent at varying cadences (stepping, low cadence walking, high cadence walking). Methods: Verification Seventy-eight participants wore a thigh mounted activPAL and wristmounted GENEActiv over a week in free-living conditions. The activPAL data were used as the criterion measure. Analytical Validation - A method for analysing raw accelerometer data was developed to optimise the agreement between daily step totals measured at the wrist and thigh. Clinical Validation - In order to examine how the algorithm generalised to unknown participants, the digital biomarkers of cadence were calculated for 713 people aged 65-98. Correlations were computed between the biomarkers and a 4-meter gait speed test. Results: Applying a zerocrossing counter with a $100 \mathrm{mg}$ hysteresis $(+/-50 \mathrm{mg})$ to filtered acceleration data led to optimal performance. Step counts from the wrist-worn devices differed by $6 \%$ (650 steps) from the activPAL, showing a high degree of accuracy. The average F1-score of the cadence measures was 0.89 (SD 0.082 ). In the 713 participants, a correlation of $0.37(p<0.001)$ was found between time spent in 'high cadence walking' and 4-meter walk times, explaining $13.6 \%$ of the variance. Discussion: It is possible to create an algorithm that can translate acceleration gathered from a research-grade, wrist-worn accelerometer into digital biomarkers of step cadence with a high degree of accuracy. Furthermore, these cadence measures correlate with gait speed tests in older adults, indicating the clinical validity of such biomarkers. 


\section{Measurement \& Analysis: Multi-Modal Assessment}

Freezing of Gait Among Patients With Parkinson's Disease Measured During Daily Living: Associations With Self-Report Questionnaires and Structured Provoking Tests

Diana Denk ${ }^{1}$, Talia Herman ${ }^{1}$, Demi Zoetewei ${ }^{2}$, Pieter Ginis ${ }^{2}$, Marina Brozgol $^{1}$, Pablo Cornejo Thumm ${ }^{1}$, Irina Galperin ${ }^{1}$, Eva Decaluwe ${ }^{2}$, Natalie Ganz ${ }^{1}$, Luca Palmerini ${ }^{3}$, Nir Giladi ${ }^{1}$, Alice Nieuwboer ${ }^{2}$, Jeffrey Hausdorff ${ }^{1}$

${ }^{1}$ Tel-Aviv Sourasky Medical Center, ${ }^{2}$ KU Leuven, ${ }^{3}$ University of Bologna

Introduction: Freezing of Gait (FOG) is an episodic phenomenon that is common among patients with advanced Parkinson's disease (PD). FOG markedly reduces quality of life and increases fall risk. Two approaches have been used to quantify FOG: self-report questionnaires and the percent time frozen (\%TF) during FOG-provoking, structured tests (e.g., walking in a tight circle or turn). More recently, the \% TF during unsupervised, community ambulation has been proposed to measure FOG as patients carry out their routine activities of daily-living. Here we evaluate the association between this new measure of FOG and previously studied measures and related factors. Methods: Data from 28 people with PD and FOG were assessed clinically. They completed structured FOG-provoking tests, both OFF and ON dopaminergic medications, and two previously validated FOG Questionnaires (i.e., total of 44 outcomes). Subsequently, they wore a 3D accelerometer and gyroscope on each shoe for 1 week as they carried out their routine activ ities. Based on the $\% \mathrm{TF}$, during daily-living subjects were divided (median split) into those with mild ( $\mathrm{n}=14<1 \% \mathrm{TF})$ and moderate $(\mathrm{n}=14>1 \%$ TF) FOG. Result: Out of 44 outcomes, only 5 differed in those with mild and moderate daily-living FOG (Figure 43). For example, in the OFF (but not $\mathrm{ON}$ ) medication state, \% TF during the four-meter walk and the Timed up and Go test with dual-task was lower in subjects with mild daily-living FOG, compared to those with moderate daily-living FOG. The two groups were similar in age, disease duration and scores on both selfreport tests of FOG. Discussion: The present findings suggest that "reallife" $\% \mathrm{TF}$ is related to a subset of clinical measures and that it reflects FOG-provoked in the OFF medication state, while it is not associated with self-report of FOG or FOG-provoking tested in the ON state. Dailyliving measurement provides a complementary and informative approach to the objective evaluation of FOG.

Joint and Individual Variations of Sleep, Physical Activity and Circadian Rhythmicity Features in CoLaus Study

Sun Kang ${ }^{1}$, Andrew Leroux ${ }^{2}$, Wei Guo ${ }^{1}$, Martin Preisig ${ }^{3}$, Kathleen Merikangas ${ }^{1}$, Vadim Zipunnikov ${ }^{4}$

${ }^{1}$ National Institute of Mental Health, ${ }^{2}$ University of Colorado, ${ }^{3}$ University Hospital of Lausanne, ${ }^{4}$ Johns Hopkins Bloomberg School of Public Health

Introduction: There is now growing evidence from dysregulation of sleep (SL), physical activity (PA) and/or circadian rhythms (CR) in people with major depression (MDD). However, few studies of large population cohorts have examined associations between the full range of features that can be derived from actigraphy. Here, we examine the interaction and inter-correlations among the three major domains (SL, PA and CR) extracted from actigraphy using a machine learning approach to quantify their joint and individual variation. Methods: The sample included 2317 participants from the CoLaus study, a prospective cohort study from the general population of Lausanne, Switzerland. Clinical diagnoses were obtained through a comprehensive diagnostic interview to ascertain lifetime history of mood and other disorders. There are a total of 1153 people with MDD and 1164 with no history of MDD. Results: The mean age is 61.79 years (range: 45 to 86 years), and $54.42 \%$ of participants are female. Features of SL, PA and CR were assessed from using Actigraphy collected with a wrist-worn triaxial accelerometer for an average of 12 days. JIVE (joint and individual variation explained) method was applied to derive the joint and individual variance of the features in SL, PA and CR. Findings indicate that the greater amount of joint variation was explained by PA and lower amounts by SL and CR. Regression analyses of the JIVE components show that participants with MDD differed significantly from controls on the first and second joint JIVE scores, but not on the individual JIVE scores. Discussion: These results demonstrate how the JIVE method enabled us to examine the joint and individual components of the actigraphy domains of SL, PA, and CR. JIVE regression allowed us to separate domain-specific sources of variability while addressing possible multicollinearity. We show that MDD was more strongly associated with joint rather than individual JIVE scores for each domain that have been the focus of most prior research in the field.

Joint Kinematics and Reaction Forces in Upside-Down Dog Position: Quantitative Differences Among Yoga Practitioners

Raffaele Zinno ${ }^{1}$, Stefano Di Paolo ${ }^{1}$, Erika Pinelli ${ }^{1}$, Giuseppe Barone ${ }^{1}$, Laura Bragonzoni ${ }^{1}$

\section{${ }^{1}$ University of Bologna}

Introduction: The efficacy of the yoga exercises could rely on the experience of the practitioner: beginners are likely to actuate negative postural compensation, thus overloading the joints. Easier variants of the yoga positions can be adopted to reduce the stress on the joints. The aim of this study was to assess differences in joint kinematics and ground reaction force on hands between yoga practitioners of different experience levels in the execution of the "upside-down dog" position, and its variants. Methods: Three men were enrolled: advanced, intermediate, beginner. Upper body kinematics was collected through a set of 15 wearable inertial sensors and ground reaction forces were collected through a force platform placed under the hands. Variants of the upside-down dog position were applied by changing height of the support under the hands: from $90 \mathrm{~cm}$ (easiest position, EP) to $0 \mathrm{~cm}$ (hardest position, HP), in $10 \mathrm{~cm}$-step for each variation. Results: From EP to HP, the beginner showed an always more posterior pelvic tilt $\left(23.5^{\circ}\right.$ excursion), while the intermediate and the advanced remained stable and neutral $\left(6.5^{\circ}\right.$ and $4.7^{\circ}$ of excursion, respectively). The shoulder flexion angle decreased of $33.5^{\circ}$ for the beginner, of $23.0^{\circ}$ for the intermediate and of $13.5^{\circ}$ for the advanced. The vertical force component on hands increased from EP to HP for all the subjects, as well as anterior force. At the HP, the vertical forces were $41.5 \%, 33.0 \%$ and $33.2 \%$ and the anterior forces were $15.5 \%, 21.0 \%$ and $23.6 \%$ of the body weight respectively for the beginner, intermediate and advanced. Discussion: When the variants became harder, the beginner was not able to maintain a neutral pelvic angle. The posterior pelvic tilt causes an anterior translation of the center of mass, and along with the reduced shoulders flexion angles, may cause an overload of the upper body chain. Harder variants of the position reflected important differences in joint kinematics and forces. (Figure 44)

Sleep Measurement Using Wrist-Worn Accelerometer Data Compared to Polysomnography

John Chase ${ }^{1}$, Michael Busa ${ }^{1}$, John Sirard ${ }^{1}$

${ }^{1}$ University of Massachusetts Amherst

Introduction: Accurate assessment of sleep and physical activity with one wearable device would decrease participant burden and provide quantitative assessment of vital health behaviors in large populations. Purpose: To determine if using alternative sleep onset (SO) definitions 
impacts accelerometer-derived sleep measurements, compared with polysomnography. Methods: Nineteen participants $(45 \% \mathrm{~F})$ completed a 48-h visit in a home simulation laboratory. Sleep characteristics were calculated for both nights of the stay by polysomnography (PSG) and a wrist-worn ActiGraph GT3X+ (AG). Criterion sleep measures included PSG-derived Total Sleep Time (TST), Sleep Onset Latency (SOL), Wake After Sleep Onset (WASO), Sleep Efficiency (SE), and Efficiency Once Asleep (SE_ASLEEP). Analogous variables were derived from temporally-aligned AG data using the Cole-Kripke sleep scoring algorithm. For PSG, SO was defined as the first score of 'sleep.' For AG, SO was defined three ways: 1,5 , and 10 consecutive minutes of 'sleep'. Agreement statistics and linear mixed effects regression models were used to interpret interactions and main effects of 'Device' (PSG vs. AG) and 'Sleep Onset Rule' (AG; 1, 5, 10-min). Results: Regression models indicated no significant interactions or main effects of 'Sleep Onset Rule' for any variable. The AG underestimated SOL (19.7 min) and WASO (7.9 min), which contributed to overestimation of TST (26.2 $\mathrm{min})$. Due to overestimation of TST, the AG also overestimated SE $(1.9 \%)$, and SE_ASLEEP (2.3\%). Sleep-wake agreement and sensitivity for PSG with all AG methods were high (89.0-89.5\% and $97.2 \%$, respectively), while specificity was low (23.6-25.1\%). Discussion: The AG under-estimation of SOL was the primary source of the overestimation of TST. Therefore, future research should focus on development of new sleep-wake detection algorithms with more sophisticated machine learning techniques and/or additional biometric signals (e.g., heart rate).

\section{The Effect of Including and Excluding Postural Status in Estimates of Sedentary Behavior}

Nicholas Lamoureux ${ }^{1}$, Gregory Welk ${ }^{1}$

${ }^{1}$ Iowa State University

Introduction: Sedentary behavior (SB) is characterized as any waking behavior with an energy expenditure below 1.5 METs performed in a seated or reclined posture. While many accelerometer devices capture the energy expenditure component, the postural component is often not fully considered. The purpose of this study was to evaluate differences in observed SB when computed with and without consideration of the postural status. Method Data were obtained from the FLASH (Free Living Activity Study for Health) which captures free living data on participants wearing multiple monitors over a 24-hour period. This study uses only records from the ActivPAL since it provides a robust indicator of posture (differentiating between sitting and standing). The ActivPAL data were processed to a second-by-second level using an open-source R package. After removing periods of non-wear, total time spent in SB was quantified using MET values only (SB1: EE $<1.5 \mathrm{METs}$ ), or MET values in combination with activPAL posture assessments (SB2: EE $<1.5 \mathrm{METs}$ and sitting/laying posture). Periods where posture indicated standing, stepping, or cycling were classified as non-sedentary, regardless of MET estimate. Results Data were processed from 130 participants (63\% female; age: $26.4 \pm 10.8$ years, BMI: $26.0 \pm 6.0$, wear time: $1413.9 \pm 46.9)$. The SB estimates were moderately correlated $(r=0.61)$, but differed considerably in magnitude. The inclusion of the postural requirement resulted in estimated SB decreasing from 1300.19 minutes (SB1) to 1087.72 minutes (SB2). Differences ranged from 45 to 599 minutes with an average absolute deviation of $21.4 \%$. Bland Altman plots show that bias varies across the range of SB values. Conclusion The results document large differences in estimated SB when posture is considered in the determination. Appropriate categorization is important for studies examining lifestyle patterns since periods of Light activity may be inappropriately coded as SB by standard accelerometers. (Figure 45)

\section{The Geosocial Observation Method for Studying Organized Groups and Group Physical Activity Outcomes for Children}

Michaela Schenkelberg ${ }^{1}$, Ann Essay ${ }^{2}$, Marisa Rosen ${ }^{2}$, Chelsey Schlechter $^{3}$, Mary Von Seggern ${ }^{1}$, Richard Rosenkranz ${ }^{4}$, David Dzewaltowski ${ }^{2}$

${ }^{1}$ University of Nebraska Omaha, ${ }^{2}$ University of Nebraska Medical Center, ${ }^{3}$ University of Utah, ${ }^{4}$ Kansas State University

Introduction: Children increasingly spend time and accumulate physical activity (PA) in adult-led organized group settings (e.g., classrooms, afterschool programs, clubs, youth sports). This study describes The Geosocial Observation Method (G-O Method) for reliably observing a group as a geographic and socially (geosocial) arranged, dynamic, timesegmented unit while simultaneously assessing group PA. Methods: A sample of organized groups $(n=66)$ nested within two communities from Wellscapes, a whole-of-community randomized controlled trial, participated in this study from 2018-2020. The G-O Method defined a geographic space for obtaining direct observation data through video for each sampled meeting day. A coding protocol was applied to time segment the meeting day routine into stable, replicable social units defined as sessions, with episodes nested within. A start/stop time boundary for each unit was defined by the location and session purpose (e.g., active recreation). Nested within sessions, episode start/stop time boundaries were defined and coded for purpose (e.g., warm-up), member arrangement (e.g., small group), and whether the segment fostered participation or exclusion (setting demand). Group PA was assessed using ActiGraph accelerometers worn by children for the duration of the group meetings. Results: Observations of group meetings $(n=231)$ yielded 940 session units and 1253 episode units. Group-level PA data were based on 1045 children across all groups. Interrater reliability was assessed on a sample of 40 group meetings ( $\mathrm{n}=162$ sessions, $\mathrm{n}=119$ episodes). Percent agreement for sessions (start/stop time, purpose) and episodes (start/stop time, purpose, member arrangement, setting demand) ranged from $82-100 \%$ and $80-100 \%$, respectively. Discussion: The G-O Method is reliable for characterizing the geographic, social, and temporal boundaries of organized groups. Paired with accelerometry, this method assesses geosocial units and group PA outcomes. (Figure 46)

\section{Using Mobile Technologies to Investigate Impaired Sleep, Mood, and Energy as Real-Time Triggers of Migraine}

Debangan Dey ${ }^{1}$, Tarannum Lateef, Kathleen Merikangas ${ }^{2}$, Andrew Leroux $^{3}$, Vadim Zipunnikov ${ }^{1}$, Mike Xiao

${ }^{1}$ Johns Hopkins Bloomberg School of Public Health, ${ }^{2}$ National Institute of Mental Health, ${ }^{3}$ University of Colorado

Introduction: Migraine headaches are highly prevalent across the lifespan and often associated with significant functional impairment, yet migraine remains underdiagnosed and undertreated. In this study, we modelled realtime associations between domains associated with migraine headaches including impaired sleep, mood and energy, using mobile technologies. Our main objectives were - (1) to identify whether poor sleep or prior day changes in average mood and energy states are associated with incident morning headache, (2) to evaluate whether those with a history of comorbid mood disorders have greater incidence of morning headaches. Methods: A sample of 476 participants from the National Institute of Mental Health Family Study of Affective Spectrum Disorders was used. Participants completed electronic momentary assessments (EMA)s four times a day over a two-week period. We investigated associations for AM and PM headache separately via a series of generalized linear mixed effects models adjusted for the potential confounders: age (years), sex, history of anxiety or mood (bipolar I/II, major depressive) disorders, history of migraine, and weekend versus weekday. Our models account for both 
the average level of emotional states and sleep across the two weeks as well as within-subject normalized changes in these states on the previous day. Results: We discovered statistically significant associations between AM headache and both the average sleep quality and the change from average sleep quality on the previous night. The effect of migraine history was stronger in case of AM headache compared to PM headache. We find an opposite directional effect of average energy and change from average energy on the previous day on PM headache incidence. Discussion: We show that assessing migraine triggers in real time using mobile technology allows us to better predict headache incidence.

\section{Measurement \& Analysis: Statistics}

\section{A Comprehensive Statistical Analysis Framework for Validation of Digital Mobility Outcomes}

M. Encarna Micó Amigo ${ }^{1}$, Anne-Elie Carsin ${ }^{2}$, Sarah Koch $^{2}$, Tecla Bonci ${ }^{3}$, Andrea Cereatti ${ }^{4}$, Rana Zia Ur Rehman ${ }^{1}$, Cameron Kirk ${ }^{1}$, Lynn Rochester $^{1}$, Aida Aydemir ${ }^{5}$, Claudia Mazzà ${ }^{3}$, Judith Garcia-Aymerich ${ }^{2}$, Silvia Del Din ${ }^{1}$

${ }^{1}$ Newcastle University, ${ }^{2}$ Barcelona Institute for Global Health (ISGlobal), ${ }^{3}$ Sheffield University, ${ }^{4}$ Politecnico di Torino, ${ }^{5}$ a business of Merck KGaA, Darmstadt, Germany

Introduction: Quantifying digital mobility outcomes (DMOs, e.g. walking speed) with wearable sensors (WS) requires the implementation of algorithms from which DMOs are extracted. To ensure DMOs are valid they need to be compared to the reference values, as provided by gold standard technology (e.g., walkway system), however standard methods do not exist. In this study, a comprehensive statistical framework to evaluate DMOs criterion validity was developed and implemented. Using this framework a worked example describing performance metrics and statistical methods for spatiotemporal DMOs was presented. Methods: Using available datasets within the scientific Mobilise-D consortium, 2 we developed and implemented a framework to validate a heterogeneous set of spatiotemporal DMOs (Figure 47). Criterion validity of DMOs derived from a single WS was determined by comparison to a corresponding reference system. Results: We applied the statistical framework to DMOs of interest and agreed within Mobilise-D: start, end and duration of detected gait sequences (i.e. "walking" activity); identification and laterality (left/right) of initial contact events; step duration; stride length; cadence; walking speed; angle, duration, start and end of turns. These DMOs were measured in both laboratory and real-world contexts, in healthy controls, people with Parkinson's disease, stroke survivors, people with choreic movement disorders and people with Multiple Sclerosis. The proposed performance metrics and statistical methods were performed for each DMO by cohort. Discussion: We provided a comprehensive statistical framework to evaluate the criterion validity of DMOs. We propose this framework could be adopted as a tool to standardise DMO validation and facilitate analyses of DMOs in future studies. Moreover, this framework should be considered by professionals, researchers, and engineers and can be implemented in other fields where evaluation of the validity of outcomes is necessary.

\section{Agreement of Step Metrics Derived From ActiGraph and activPAL} Accelerometers Worn Concurrently Among Older Adults

Eric Hyde ${ }^{1}$, Steve Nguyen ${ }^{2}$, Mikael Anne Greenwood-Hickman ${ }^{3}$, Andrea LaCroix $^{2}$, Christopher Moore ${ }^{4}$, Loki Natarajan ${ }^{2}$, Dori Rosenberg ${ }^{3}$, Fatima Tuz-Zahra $^{2}$, Rod Walker ${ }^{3}$, John Bellettiere ${ }^{2}$

${ }^{1}$ San Diego State University/University of California, San Diego, ${ }^{2}$ University of California, San Diego, ${ }^{3}$ Kaiser Permanente Washington Health Research Institute, ${ }^{4}$ University of North Carolina, Chapel Hill
Introduction: The agreement of step-based metrics across various wearable devices is a known research gap. Our study evaluated the agreement of daily step counts, peak 1-min cadence, and peak 30-min cadence between the hip-worn ActiGraph (AG) GT3X+ accelerometer, using both the normal extension (AGNE) and the low frequency extension (AGLFE), and the thigh-worn activPAL 3 micro (AP) monitor in a sample of US older adults. Methods: 953 older adults enrolled in the Adult Changes in Thought Study who wore the AG concurrently with the AP for 4-7 days beginning in 2016. Using the AP as the reference measure, device agreement was assessed using mean error (AGNE-AP and AGLFE-AP) and $95 \%$ limits of agreement, mean absolute percentage error (MAPE), Pearson correlations, and concordance correlations. For each step metric, distributions were plotted as histograms. Results: For AGNE-AP, the mean error and MAPE were: daily steps $-1851(-3799,97)$ and $27.2 \%$ steps/day, peak 1-min cadence $-16.2(-46.0,13.7)$ and $16.3 \%$ steps $/ \mathrm{min}$, and peak 30 -min cadence $-17.7(-38.7,3.3)$ and $24.0 \%$ steps/min. Pearson correlations were $0.94,0.85$, and 0.91 and concordance correlations were 0.81 , 0.65 , and 0.73, respectively. For AGLFE-AP, the mean error and MAPE were: daily steps $4968(1629,8307)$ and $72.7 \%$ steps/day, peak 1-min cadence $-1.4(-13.7,10.9)$ and $4.7 \%$ steps $/ \mathrm{min}$, and peak 30 -min cadence $1.4(-9.6,12.5)$ and $7.0 \%$ steps $/ \mathrm{min}$. Pearson correlations were $0.91,0.91$, 0.95 and concordance correlations were $0.49,0.91$, and 0.94 , respectively. Discussion: Overall, our results suggest that discrepancies exist in daily step estimates across the AGNE, AGLFE, and AP (MAPE > 25\%), and step cadence estimates between the AGNE and AP. Further research is needed to clarify whether these discrepancies impact investigations of the associations between step-based metrics and health outcomes. (Figure 48)

\section{Associating Aging With Actigraphy-Based Walking Features Ex- tracted Via Structured Functional Principal Components}

Verena Werkmann ${ }^{1}$, Nancy Glynn ${ }^{2}$, Jaroslaw Harezlak ${ }^{1}$

${ }^{1}$ Indiana University, ${ }^{2}$ University of Pittsburgh

Introduction: As walking is the most accessible form of physical activity, one's physical health is likely related to walking features. However, current methods extracting walking features from raw accelerometry data do not account for varying cadence and commonality of features among subjects. Methods: We use data collected using ActiGraph GT3X+devices (sampling $=80 \mathrm{~Hz}$ ) as part of the Developmental Epidemiologic Cohort Study (DECOS), $\mathrm{N}=48$, age $=78.7 \pm 5.7$ years, $46.8 \%$ women. We apply structured functional principal component analysis (SFPCA) to extract features from walking signals on both population- and subjectspecific levels from a fast-paced 400m walk, an indicator of aerobic fitness in older adults. We also use the population level feature scores to study their associations with age and physical performance measures (gait speed and Short Physical Performance Battery, SPPB). Specifically, we transform raw data into the frequency domain by applying local Fast Fourier Transform to obtain the walking spectra. SFPCA decomposes these spectra into easily interpretable walking features expressed as cadence and acceleration, which can be related to physical performance, potentially shedding light on subclinical disease. Results: We found that 7 population level and 39 subject-specific level features explained more than $95 \%$ of their respective level variation, thus significantly reducing the complexity of the data. Our results show that $68 \%$ of the total data variation arises at the population level and $32 \%$ at the subject-specific level. Moreover, we found that lower acceleration magnitude at the cadence was associated with older age, slower gait speed, and worse SPPB scores. Higher acceleration magnitude at cadence multiples of 2.5 and 3.5 were only associated with faster gait speeds. Discussion: SFPCA extracted population- and subjectspecific empirical walking features in the frequency domain which were meaningfully associated with a number of health indicators and older age. 
Comparison of Accelerometer Daily Minimum Wear Time in Healthy Children and Children With Inflammatory Bowel Disease

Elizabeth Ball ${ }^{1}$, Madelyn Byra ${ }^{1}$, Brian Timmons ${ }^{1}$, Joyce Obeid ${ }^{1}$

${ }^{1}$ McMaster University

Introduction: Accelerometers have been used to estimate habitual physical activity (PA) and sedentary time (SED) in children with inflammatory bowel disease (IBD). It is not clear if minimum wear time criteria for estimating PA and SED in healthy children applies to IBD. Unpredictable patterns of disease activity and frequent hospital visits in IBD may impact the stability of movement behaviours. The objective of this study was to compare the minimum accelerometer wear time to achieve reliable activity estimates in IBD and healthy controls. Methods: Children with IBD $(\mathrm{N}=17)$ and healthy controls $(\mathrm{N}=15)$ wore an ActiGraph GT3X accelerometer during waking hours for 7 days. Outcomes of interest included axis 1 counts per minute (cpm), vector magnitude cpm, sedentary time, time spent in light (LPA), moderate-to-vigorous (MVPA), and total physical activity (TPA), determined using Evenson cut-points. Dayto-day reliability coefficients (r) were calculated using Spearman-Brown formula based on the intraclass correlation coefficient. Acceptable reliability was set at $r \geq 0.8$. Only participants with $\geq 1 \mathrm{hr}$ of data on $\geq 3 \mathrm{~d}$ were included in the analysis. Results: Children with IBD achieved acceptable reliability for axis $1 \mathrm{cpm}(\mathrm{r}=0.834)$, vector magnitude cpm $(\mathrm{r}=0.86)$, sedentary time $(\mathrm{r}=0.854)$, LPA $(\mathrm{r}=0.957)$, and TPA $(\mathrm{r}=0.878)$ after 3 $\mathrm{d}$ of wear, and after $4 \mathrm{~d}$ for MVPA $(\mathrm{r}=0.821)$. Healthy children achieved acceptable reliability after $3 \mathrm{~d}$ for sedentary time $(\mathrm{r}=0.818), 4 \mathrm{~d}$ for LPA $(\mathrm{r}=0.876), 6 \mathrm{~d}$ for vector magnitude $\mathrm{cpm}(\mathrm{r}=0.833)$, and $7 \mathrm{~d}$ for axis $1 \mathrm{cpm}(\mathrm{r}=0.818)$ and MVPA $(\mathrm{r}=0.827)$. Discussion: Minimum wear time criteria to estimate PA, and specifically MVPA, is shorter in IBD compared to healthy controls (4 vs. 7 days), but similar for SED. It is plausible that PA in children with IBD is already low and limited by their condition, resulting in less day-to-day variability. Healthy children may have a wider range of activities thus increasing the days needed to reliably estimate PA.

Comparison of Accelerometry-Derived Physical Activity Summary Measures By Age, Sex, and BMI

John Muschelli ${ }^{1}$, Andrew Leroux ${ }^{2}$, Jacek Urbanek ${ }^{1}$, Amal Wanigatunga ${ }^{1}$, Jiawei Bai ${ }^{1}$, Ciprian Crainiceanu ${ }^{1}$, Jennifer Schrack ${ }^{1}$

${ }^{1}$ Johns Hopkins University, ${ }^{2}$ University of Colorado

Introduction: Open-source summaries of raw, sub-second level acceleration data have recently been proposed, including Monitor Independent Movement Summary (MIMS), Euclidean Norm Minus One (ENMO), Mean Amplitude Deviation (MAD), Activity Index (AI). However, the correlation between these summaries and the widely used ActiGraph activity counts (AC) has not been assessed. Methods: We used data from 655 participants in the Baltimore Longitudinal Study on Aging (BLSA) who wore a wrist-worn Actigraph GT9x continuously for a week. We further: (1) provided unified R software to aggregate raw acceleration data using MIMS, ENMO, MAD, AI; (2) estimated the correlations between $\mathrm{AC}$ and open-source statistics, and explored whether they differed by age, sex and BMI; and (3) estimated a mapping between AC and open-source measures, which can be used to harmonize measurements. Results: At the time of accelerometer wear, study participants: had a mean (sd) age of 69.8 (14.2) years and BMI of 27.3 (5.0), were 54.5\% females, $67.9 \%$ were white and $95.9 \%$ reported good to excellent health. Participant's daily total AC averaged 2,219,472. Estimated participant-specific correlation between AC and: MIMS, ENMO, MAD, and AI had a mean of 0.988, 0.733, 0.913 and 0.970 , respectively. With the exception of ENMO, there were no covariate-specific correlation patterns (see for age in Figure 49). The derived mapping between AC and: MIMS, ENMO, MAD, and AI allowed to translate existing thresholds of PA expressed in AC into units of the open-source measures. Discussion: We quantified correlations and derived mappings between minute-level ActiGraph AC and open-source statistics. Our work will enhance the comparability of results and facilitate the harmonization of data across research studies. This research is especially timely given the recent release of PA data from NHANES 2011-2014 that uses MIMS.

\section{Distributional Data Analysis Via Quantile Functions and its Applica- tion to Modelling Digital Biomarkers of Gait in Alzheimer's Disease}

Rahul Ghosal ${ }^{1}$, Vijay R. Varma ${ }^{2}$, Dmitri Volfson ${ }^{3}$, Inbar Hillel ${ }^{4}$, Jacek Urbanek $^{5}$, Jeffrey M. Hausdorff ${ }^{4}$, Amber Watts ${ }^{6}$, Vadim Zipunnikov ${ }^{1}$

${ }^{1}$ Johns Hopkins Bloomberg School of Public Health, ${ }^{2}$ National Institute on Aging (NIA), National Institutes of Health (NIH), ${ }^{3}$ Neuroscience Analytics, Computational Biology, Takeda, ${ }^{4}$ Tel-Aviv Sourasky Medical Center, ${ }^{5}$ Johns Hopkins University, ${ }^{6}$ University of Kansas

Introduction: With the advent of continuous health monitoring via wearable devices, users now generate their unique streams of continuous data such as minute-level physical activity or heart rate. Aggregating these streams into scalar summaries ignores the distributional nature of data and often leads to the loss of critical information. Methods: We propose to capture the distributional properties of wearable data via user-specific quantile functions that are further used in functional regression and multimodal distributional modelling. In addition, we propose to encode userspecific distributional information with user-specific L-moments, robust rank-based analogs of traditional moments. Importantly, this L-moment encoding results in mutually consistent functional and distributional interpretation of the results of scalar-on-function regression. We also demonstrate how L-moments can be flexibly employed for analyzing joint and individual sources of variation in multi-modal distributional data. Results: The proposed methods are illustrated in a study of association of accelerometry-derived digital gait biomarkers with Alzheimer's disease (AD) and in people with normal cognitive function. Our analysis shows that the proposed quantile-based representation results in a much higher predictive performance compared to simple distributional summaries and attains much stronger associations with clinical cognitive scales. Discussion: Distributional modelling of wearable data can help to identify individuals at-risk for developing dementia within large, population-based studies.

\section{Effect of Physical Activity on PPG Signal Quality}

Serena Moscato ${ }^{1}$, Lorenzo Chiari ${ }^{1}$

${ }^{1}$ University of Bologna

Introduction: Photoplethysmographic (PPG) sensors allow the detection of blood volume changes non-invasively. The signal has a stereotyped waveform (i.e., PPG pulse) which occurs within each heartbeat. PPG is mainly used for heart rate (HR) estimation, but several studies proved that a deeper morphological analysis (MA) could provide relevant parameters related to the user's cardiovascular state. PPG sensors are highly susceptible to motion artifacts, lowering the reliability of the derived parameters. We aim to assess the impact of physical activity on the quality of PPG signals recorded in a free-living context. Methods: Thirty-one subjects were monitored for 24 hours with the Empatica E4 wristband. An Activity Index (AI) was computed from the accelerometer data and associated with each PPG pulse. We defined four activity levels based on the quartiles of the AIs distribution. Within each recording, 100 PPG pulses from each activity level were randomly selected and labeled by three independent annotators through a custom Matlab GUI into three quality classes: bad 
(noise only), basic (valid for HR estimate), and diagnostic (valid for MA). Quality classes and AIs are negatively correlated $(\mathrm{r}=-0.6093$, $\mathrm{p}<0.01)$. Results: The four activity levels correspond respectively to rest/sleep, sedentary, light, and light-moderate activity. The percentages of PPG pulses with basic and diagnostic quality decrease when the activity increases (Figure 50A). Not surprisingly, the distribution of quality classes during the 24 hours highlights that the highest amount of PPG pulses with diagnostic and basic quality can be collected during the night. Still, some good pulses can also be collected during lightmoderate activity (Figure 50B). Discussion: Despite the significant impact of physical activity on PPG quality, it is still possible to detect basic and diagnostic quality PPG pulses when the user is active. This preliminary analysis shows that a prodromal quality analysis is necessary to obtain reliable information from PPG signal.

Examining Relative Intensity Estimates From Fitbit-Derived Heart Rate in Exercise Phenotypes: Understanding Where the Errors Live

Megan Heintzelman ${ }^{1}$, Kyle Winfree ${ }^{2}$, Matthew Saponaro ${ }^{3}$, Richard Suminski ${ }^{1}$, Gregory Dominick ${ }^{1}$

${ }^{1}$ University of Delaware, ${ }^{2}$ Northern Arizona University, ${ }^{3}$ AI Whoo

Introduction: Wearables such as Fitbit underestimate HR compared to criterion measures. Yet few studies have explored how this bias affects relative intensity estimates. This study examined the variation of HR error by exercise phenotype across the intensity spectrum. Methods: Participants $(\mathrm{N}=19)$ wore a Fitbit Charge HR (FB) and Polar H7 (PH7) HR monitor for 7 days while recording the type and duration of exercise lasting $>10 \mathrm{~min}$. via a study app. Minute-level HR data were calculated using the percent heart rate reserve method (\%HRR $=30-39 \%, 40-59 \%$, $\geq 60 \%$ ), reflecting light, moderate, and vigorous intensity, respectively. Exercises were grouped into phenotypes by similarity using a Euclidean distance between all possible pairings on axes of time in different intensity levels. A threshold metric was determined using a detrended fluctuation analysis on the number of resultant groups for each unique distance measured $\left(12^{\wedge} 2\right)$. We then determined the mean and standard deviation of the error, as well as the variance between FB and PH7 \%HRR at each intensity level among exercise phenotypes. Inter-device differences were characterized as mean $\pm \mathrm{SD}$, congruence with ICCs, and localization of inter-device \%HRR differences with Bland-Altman. Results: A total of 3,963 min of activity was recorded for 12 exercise types, grouped into 7 mutually exclusive phenotypes (walk, run+elliptical, weights+yoga, spin+sport+HIIT, exercise class, pilates, bike+crossfit). Overall, as \%HRR increases, the error variance decreases from light to moderate, and from moderate to vigorous intensity (Figure 51). HR error was lowest for run+elliptical $(7.27 \%)$ and walk $(7.77 \%)$ and highest in weights+yoga (17.86\%) and spin+sport+HIIT (15.57\%). Discussion: Activity type contributes to the overall variance and error of relative intensity estimates derived from FB. Ambulatory exercise (e.g., walk and run) provide more accurate relative intensity estimates versus intermittent exercise (e.g. weights).

Factors Contributing to the Use of Wearables and Subsequent Engagement in Protective Health Behaviour

Ruhi Bajaj ${ }^{1}$, Rebecca Meiring ${ }^{1}$, Fernando Beltran ${ }^{1}$

${ }^{1}$ University of Auckland

Introduction: Wearable technology, in the form of activity trackers and smartwatches, motivates people to remain active by providing real-time data about their physical activity along with digital biomarkers that may result in people making decisions to protect their health. This study aimed to determine the relationship between health risks and the benefits of knowing family medical history on the use of wearables and subsequent engagement in protective health behaviour. Methods: Health risk (perceived vulnerability/perceived severity) is the likelihood or seriousness of an adverse health consequence due to a specific disease or condition. Protective health behaviour is one's level of engagement in behaviours that are beneficial to health, such as regular exercise, healthy eating, and using wearables for continuous health monitoring. Wearable devices act as 'coping responses' to overcome health risks, and response cost indicates the time, money, and effort invested in using these devices. Self-efficacy refers to one's ability to use wearables competently to attain various health goals. An online survey was conducted, involving wearable users over 18 years of age $(n=300 ; 57 \%$ male $)$ recruited through Amazon MTurk, to analyze protective health behaviours using Structural Equation Modeling. Results: This study found that the perceived benefits of knowing family medical history fully mediates the relationships of health risks with protective health behavior as shown in Figure 52. In addition, self-efficacy influenced the protective health behaviour, but response cost did not. Discussion: Individuals who perceive themselves as being at high risk of health threats and believe that there are benefits of knowing their family medical history are more likely to use wearables to protect their health. These people strongly believe that their family health patterns drive their health behaviours and outcomes. They also feel competent to use wearables to attain various health goals.

\section{Frequency and Severity of Device-Related Complaints, Overall and} By Month of Year, in the ABC Study

Weng Chi Lou ${ }^{1}$, Nga Nguyen ${ }^{1}$, Theresa Whalen ${ }^{1}$, Fiona Bruinsma ${ }^{1}$, Graham Giles ${ }^{1}$, Roger Milne ${ }^{1}$, Brigid Lynch ${ }^{1}$

\section{${ }^{1}$ Cancer Council Victoria}

Introduction: The Australian Breakthrough Cancer (ABC) Study is an Australian cohort investigating how genes and lifestyle influence the risk of cancer. A sub-sample of participants were recruited to an accelerometer study. Here, we describe the frequency and severity of device-related complaints, overall and by month of year. Method: We invited 7,737 eligible ABC Study participants to participate in the Accelerometer Study. Participants wore two devices (Actigraph GT3X+ on elasticised waist band; and activPAL adhered to thigh with Nexcare Tegaderm waterproof dressing) for 7 consecutive days. Actigraphs were worn during waking hours; activPALs were worn 24 hours per day. We characterised complaints as: mild (not sufficient to cause discontinued use of device); moderate (discomfort resulting in discontinued use, switching leg or position, or using alternative adhesive method); and severe (painful irritation causing immediate cessation and strong complaint). Results: 4,238 participants (50\% women) consented and were sent devices. Between September 2016 and March 2020, 13 complaints were made related to the waist-worn Actigraph device ( 7 mild and 6 moderate complaints), all but 1 of which related to the elasticised waistband being uncomfortable. Over the same period there were 120 complaints related to activPALs (69 mild, 38 moderate and 13 severe complaints). The most common mild complaint related to difficulty with adhesion, while moderate or severe complaints mostly related to skin irritation, allergies or blisters. A seasonal pattern was apparent for these complaints (Figure 53). Only 10\% of complaints occurred during Australia's winter months. Discussion: We achieved high compliance with study protocols, and complaints - particularly severe ones - were rare, but it is likely that not all mild or even moderate skin irritation were reported. Alternative adhesion methods to help maximise adherence to activPAL protocols during warmer months should be investigated. 
Intraindividual Variability in GPS-Derived Community Mobility Characteristics of Older Adults: Links With Physical and Cognitive Functioning

Kyle Moored ${ }^{1}$, Breanna Crane ${ }^{2}$, Michelle Carlson ${ }^{2}$, Andrea Rosso ${ }^{1}$

${ }^{1}$ University of Pittsburgh, ${ }^{2}$ Johns Hopkins Bloomberg School of Public Health

Introduction: GPS technologies allow for objective measurement of community mobility, including the area (i.e., space) and shape of one's daily outdoor activity. Existing studies focus on average GPS-derived measures, but fewer studies examine intraindividual variability (e.g., within-person deviation across days). We computed multiple measures of variability in GPS-derived activity and examined associations with in-lab performance. Methods: Participants were 164 adults (Age: $\mathrm{M}=77.3 \pm 6.5$ ) from a randomized trial to improve walking in community-dwelling older adults. Participants carried a portable GPS at baseline that passively collected real-time location data for 7 days. Areas (i.e., activity space) and compactness (i.e., activity shape) of standard deviational ellipses (SDEs) and minimum convex polygons (MCPs) were derived for each wear day. 7-day medians and median absolute deviations (MAD) for each measure were generated to capture central tendency and variability of weekly activity, respectively. Coefficients of variation (MAD/median) were also calculated to assess variability accounting for median activity. Spearman correlations tested associations with cognitive (Modified Mini Mental Status, 3MS) and physical (6-Minute Walking Test, 6MWT) function with adjustment for age and sex. Results: Activity space medians $(\rho=.17-.20$, all $\mathrm{p}<.05)$ and MADs $(\rho=.17-.18$, all $\mathrm{p}<.05)$ were positively associated with 6MWT performance. MCP MAD also had a positive association with $3 \mathrm{MS}(\rho=.20, p=.05)$. Coefficients of variation for activity space measures had trending negative associations with $6 \mathrm{MWT}$ performance $(\rho=-.14$ to -.15 , all $p=.06)$. Compactness measures were not associated with function (all $\mathrm{p}>.05$ ). Discussion: Having a large and consistent activity space appeared most related to higher physical and cognitive function. Future work will examine within-day variability in activity and whether these metrics predict future cognitive and functional health in older adults.

postGGIR: An Open Source R/R-Markdown Package for Post-GGIR Processing of Accelerometer Data

Vadim Zipunnikov ${ }^{1}$, Andrew Leroux ${ }^{2}$, Kathleen Merikangas ${ }^{3}$

${ }^{1}$ Johns Hopkins Bloomberg School of Public Health, ${ }^{2}$ University of Colorado, ${ }^{3}$ National Institute of Mental Health

Introduction: Objectively-assessed motor activity has recently received a lot of attention in mental health research. The R package GGIR is a popular tool for processing raw accelerometer data. A GGIR user must load the GGIR output, clean the data, and extract important features of interest. The flexibility and amount of user control offered by GGIR is an undeniable strength of the software. To further simplify data processing pipelines that use GGIR, we developed postGGIR, an R package that both facilitates user-friendly visualization of summary output from GGIR and streamlines the data processing pipeline, extracting features from the domains of sleep, physical activity, and circadian rhythmicity. Methods and results: postGGIR streamlines GGIR data processing by performing the following six steps. First, the output of GGIR is examined for missingness and duplicates by counting the number of files in each GGIR output folder as well as in GGIR summary files. Second, the quality of the accelerometer data is assessed using estimated missingness and non-wear time. Third, the accelerometer data that GGIR saves as separate CSV files is read, merged, transformed, aggregated, and imputed. Fourth, additional features are extracted from minute level activity data to provide more comprehensive description of domains of sleep, physical activity and circadian rhythmicity. Fifth, the features from these three domains are then decomposed into new latent features using the Joint and Individual Variation Explained (JIVE) method that provides latent factors capturing information individual to each of the three domains as well as the joint information. In the final sixth step, comprehensive.html reports of the data processing pipeline and visualization of the processed data are created using $\mathrm{R}$ Markdown. Discussion: postGGIR builds on flexibility and comprehensiveness of GGIR and provides a postGGIR processing pipeline which saves user's time.

\section{Pursuit of Ideal Timing and Distribution of Movement Behaviours for} Post-Concussion Symptoms

Nicholas Kuzik $^{1}$, Mike Borghese ${ }^{1}$, Adrienne Davis ${ }^{2}$, Gurinder Sangha ${ }^{3}$, Ken Tang ${ }^{1}$, Mark Tremblay ${ }^{1}$, Andrée-Anne Ledoux ${ }^{1}$, n/a n/a ${ }^{4}$

${ }^{1}$ Children's Hospital of Eastern Ontario Research Institute, ${ }^{2}$ Hospital for Sick Children, ${ }^{3}$ Children's Hospital London Health Sciences Centre, Western University, ${ }^{4}$ on behalf of the Pediatric Emergency Research Canada (PERC) Pediatric Concussion Assessment

Introduction: Rest is often prescribed for pediatric concussion management. However, evidence is limited for the ideal balance of rest and exertion post-concussion. Specifically, research is needed to determine the ideal timing and distribution of movement behaviours (i.e., sleep, stationary time, light-intensity physical activity [LPA], and moderate- to vigorous-intensity physical activity [MVPA]) for post-concussion symptoms. The objective of this study was to investigate the ideal distribution of movement behaviours for each day after an acute concussion. Methods: This was a secondary analysis of the PedCARE study, a Canadian multicentre randomized clinical trial. Children aged 10.00 to 17.99 years with an acute concussion were recruited. Cognitive and somatic postconcussion symptoms were measured with the Health and Behaviour Inventory (HBI) at baseline and 2-weeks. Children wore an Actical accelerometer 24-hours/day for 13 days. Sleep times were reported and verified using visual inspection of accelerometer data while other movement behaviours were classified using established cut-points. For days 113, robust regression models were built with HBI categories and isometric log-ratio movement behaviour compositions. On significant days, changes in cognitive and somatic HBI scores were predicted for all possible substitutions of movement behaviours within a 1SD range (e.g., \pm 1 SD sleep). The best $5 \%$ of predicted HBI scores were retained to calculate ideal distributions of movement behaviours for that day. Results: The composition of movement behaviours on days 6 and 13 were significantly associated $(\mathrm{p}<0.05)$ with improved cognitive and somatic HBI symptom changes (Figure 54). Discussion: Compared to the mean distribution of movement behaviours, the best days for improving postconcussion symptoms seem to have more MVPA and less sleep. Future research should examine longitudinal associations between the distribution of movement behaviours and post-concussion symptoms.

\section{Real-World Gait Effects Quantization of Orthotic Shoes on Diabetic Peripheral Neuropathy Patients Using Inertial Sensors}

\section{Phuoc Nguyen ${ }^{1}$, Mary Spires ${ }^{1}$, James Leonard ${ }^{1}$, Lauro Ojeda ${ }^{1}$}

${ }^{1}$ University of Michigan

Introduction: A common complication of Type II diabetes is distal symmetric diabetic peripheral polyneuropathy (DPN) commonly involving the feet. It can result in numbness or loss of sensation and pain in the feet. DPN frequently results in altered foot posture leading to gait alteration. Orthopedic extra-depth shoes (EDS) and these fitted with custom 
orthotic insoles (COIS) are preventive measures and treatment for reducing foot complications, however their effectiveness at mobility have not been quantized. To fulfill this gap, we propose using inertial measurement units (IMUs). Methods: We collected 3 weeks of IMU data (one with each type of shoe) from the two feet of 7 participants (1F, 6M, 68.1/2.3 $\mathrm{yr}$ avg/std) diagnosed with Type II diabetes ( $\geq 2 \mathrm{yr}$ ) and DPN ( $\geq 1$ yr). From IMU data we estimated stride speed and length, and used histograms to identify preferred stride length and speed. The differences of those values across the three types of shoes were compared using sample t-test $(\mathrm{alpha}=0.1)$ to determine statistical significance. Results: Compared to NS, patients with EDS walked using longer $(4.1 \mathrm{~cm}$ or $3.6 \%$ avg inc, $\mathrm{p}=0.072)$ and faster strides $(4.9 \mathrm{~cm} / \mathrm{s}$ or $5.1 \%$ avg inc, $\mathrm{p}=0.157)$. Comparing NS to COIS, with the latter patients showed longer $(2 \mathrm{~cm}$ or $1.9 \%$ avg inc, $\mathrm{p}=0.171$ ) and faster strides $(2.1 \mathrm{~cm} / \mathrm{s}$ or $2.4 \%$ avg inc, $\mathrm{p}=0.517)$. Comparing the two orthotic interventions, with COIS most patients walked using shorter $(2.1 \mathrm{~cm}$ or $1.6 \%$ avg dec, $\mathrm{p}=0.370)$ and slower strides $(2.8 \mathrm{~cm} / \mathrm{s}$ or $2 \%$ avg dec, $\mathrm{p}=0.522$ ). Discussion: Patients with either orthotic intervention increased stride length and speed, but only the EDS stride length increase was statistically significant. Interestingly, COIS did not seem to outperform commercial EDS in any parameter. We admit that differences in daily life environments and activities can influence the performances and these results must be further investigated under a longer period of testing and with a larger sample size. (Figure 55)

Role of Psychosocial Factors on the Effect of Physical Activity on Physical Function in Patients After Lumbar Spine Surgery

Hiral Master ${ }^{1}$, Renan Castillo ${ }^{2}$, Stephen Wegener ${ }^{2}$, Jacquelyn Pennings ${ }^{1}$, Rogelio Coronado ${ }^{1}$, Christine Haug ${ }^{1}$, Richard Skolasky ${ }^{2}$, Lee Riley III ${ }^{2}$, Brian Neuman ${ }^{2}$, Joseph Cheng ${ }^{3}$, Oran Aaronson ${ }^{4}$, Clinton Devin ${ }^{5}$, Kristin Archer $^{1}$

${ }^{1}$ Vanderbilt University, ${ }^{2}$ Johns Hopkins University, ${ }^{3}$ University of Cincinnati, ${ }^{4}$ Saint Thomas Medical Partners, ${ }^{5}$ Steamboat Orthopedic and Spine Institute

Introduction: The purpose of this study was to investigate the longitudinal postoperative relationship between physical activity (PA), psychosocial factors, and physical function (PF) in patients undergoing lumbar spine surgery. Methods: Analytic sample included 248 participants (age $=62.2$ \pm 11.9 years and $51 \%$ females) who underwent surgery for a degenerative lumbar spine condition. PA was measured using a triaxial accelerometer (Actigraph GT3X) at 6-weeks (6wk), 6-months (6M), 12-months (12M), and 24-months (24M) after spine surgery. PF (a computerized version of Patient-Reported Outcomes Measurement Information System) and psychosocial factors (pain self-efficacy, depression and fear of movement) were assessed at preoperative visit and $6 \mathrm{wk}, 6 \mathrm{M}, 12 \mathrm{M}$ and $24 \mathrm{M}$ after surgery. Structural equation modeling (SEM) techniques were utilized to analyze data and results are represented as standardized regression weights (SRW). Overall SRW was computed across 5 imputed datasets to account for missing data. The mediation effect of each psychosocial factor on the effect of PA on PF was computed. Each SEM model was tested for model fit by assessing established fit indexes. Results: The overall effect of PA (quantified as steps per day) on PF (SRW ranged from 0.08 to 0.19 , $\mathrm{p}<0.05$ ) was stronger compared to the overall effect of PF on PA (SRW ranged from non-existent to $0.14, \mathrm{p}<0.01$ to 0.3 ). These effects remained consistent after accounting for psychosocial factors in each of the mediation models. Depression and fear of movement at $6 \mathrm{M}$ mediated $3.4 \%$ and $5.4 \%$ of the effect of PA at $6 \mathrm{wk}$ on PF at $12 \mathrm{M}$, respectively. Pain selfefficacy was not a statistically significant mediator. Discussion: The findings of this study support the use of early postoperative strategies to address PA in order to improve long-term PF. Since depression and fear of movement had a very small mediating effect, further work is needed to investigate other potential mediators, e.g. pain catastrophizing and resilience. (Figure 56)

\section{Utilizing GPS-Derived Objective Technologies to Explore Community Mobility Characteristics in Older Adults}

\author{
Breanna Crane $^{1}$, Kyle Moored ${ }^{2}$, Andrea Rosso ${ }^{2}$, Michelle Carlson ${ }^{1}$ \\ ${ }^{1}$ Johns Hopkins Bloomberg School of Public Health, ${ }^{2}$ University of \\ Pittsburgh
}

Introduction: Community mobility is defined as activity outside of the home and is necessary for many independent activities of daily living. In contrast to subjective, self-reported approaches often used to capture community mobility, GPS-derived objective measures leverage passive, real-time data collection techniques with the goal of mitigating recall bias and minimizing participant burden. A key limitation of GPS technologies is limited battery life. We will discuss the challenges and opportunities of using GPS devices with battery-saving features, considerations for data processing, and associations of derived metrics with physical and cognitive performance. Methods: Community mobility was assessed objectively using GPS technologies. Measures were generated for a sample of 164 community-dwelling older adults (Mage $=77.3 \pm 6.5$ ) in a physical therapy intervention aimed at improving walking ability. We characterized community mobility with activity space metrics (e.g., standard deviation ellipse (SDE) area), timing (e.g., time outside home), and shape (e.g., SDE compactness) of one's life-space. SDE measures were time-weighted, and sensitivity analyses were performed to explore the impact of large time gaps given the battery-saving features of the GPS technologies. Results: After adjusting for age and sex, percentage of time outside of home and SDE area, but not SDE compactness, were correlated with better performance on tests of mobility (e.g., 6-Minute Walking Test: $\rho=.16-.17$, all p's < .05). Percentage of time outside of home was also associated with better executive functioning (e.g., time to complete Trail-Making Test, Part B: $\rho=-.18, p=.02$ ). Discussion: Performance on various physical and cognitive tests was significantly correlated with various GPS-derived community mobility measures. These findings will aid in understanding which community mobility measures are linked to cognitive and physical performance among community-dwelling older adults.

\section{Measurement \& Analysis: Monitoring during COVID-19}

\section{Characterizing Physical Behaviors in Individuals With Aphasia}

Delia Moore ${ }^{1}$, Sarah Millar ${ }^{1}$, Rana Abdulkhaliq ${ }^{1}$, Carina Reyes ${ }^{1}$, Michelle Gravier $^{1}$, Jennifer Sherwood ${ }^{1}$, Albert Mendoza ${ }^{1}$

${ }^{1}$ California State University East Bay

Introduction: Aphasia is an acquired language disorder resulting from brain injury that affects $25-40 \%$ of stroke survivors, an estimated 2.5 million people in the United States. Aphasia impairs the ability to read, write, speak, and understand. Consequently, aphasia has been shown to negatively impact quality of life (QoL) and reduce physical activity (PA) levels. Regular PA and decreased sedentary behaviors (SB) can slow agingassociated physical decline and improve QoL. However, there is limited research on physical behaviors of Individuals with aphasia (IWA), or the effects of PA on language and cognitive rehabilitation in this population. The present study is part of a larger study to evaluate the feasibility and effectiveness of implementing an aphasia-friendly exercise class on QoL, SB and PA, and cognitive and linguistic outcomes. Here, we seek to characterize pre-intervention behaviors in IWA participating in the larger study. Methods: Nine participants (44\% female); age mean(SD): 60 (16) years; time post-stroke: 119 (102) months; were recruited and completed 
pre-intervention QoL, cognitive, and linguistic assessments (not reported here). Aphasia severities ranged from mild to severe. Free-living, PA data was collected using activPAL 4+ (thigh-worn on most active leg) for 7consecutive days. An average of 5 valid wear days ( $\geq 4$ days with $\geq 10$ hrs. of waking wear) were examined. Results: On average, IWA accumulate less steps per day, sit for more than twice as many minutes each day, and sleep approximately $10 \%$ longer each day than healthy age-matched adults (Figure 39). Discussion: IWA may benefit from aphasia-friendly interventions to: (1) increase PA behaviors, such as daily steps, time spent stepping and standing, (2) decrease SB, such as, daily sitting, and (3) improve QoL measures.(Figure 57)

\section{Describing 24-h Activity Patterns of Adolescent Boys Before and During the COVID-19 Lockdown in New Zealand Using a 24-h Activity Recall (STAR-24)}

Meredith Peddie ${ }^{1}$, Tessa Scott ${ }^{1}$, Jillian Haszard ${ }^{1}$

${ }^{1}$ University of Otago

Introduction: Single tools that assess sleep, physical activity and screen use (the three components of $24 \mathrm{~h}$ movement guidelines) are scarce. Therefore, the objective of this study was to use the newly developed Screen Time and Activity Recall (STAR-24) to demonstrate differences in time-use between two samples of adolescent males collected before and during COIVD-19 lockdown in New Zealand. Methods: 109 adolescent (15-18 year) boys each completed the STAR-24 twice with $n=74$ completing the questionnaire before lockdown and $n=35$ completing the questionnaire during lockdown. Results: More than $50 \%$ of the sample reported gaming between 10 am and 12 noon during lockdown, transport was not reported as an activity, and activities of daily living spiked around mealtimes. Differences in estimated moderate-to-vigorous physical activity and sleep between the sample in lockdown compared to the sample before lockdown were small (mean difference ( $95 \% \mathrm{CI}$ ) 21 ( -9 to 51 ) $\mathrm{min}$ ) and 0.5 ( -0.2 to 1.2) h, respectively). While differences in sleep duration were small, sleep timing appeared to shift to later bedtimes and later wake times during lockdown. Total screen time was $2 \mathrm{~h}$ higher ( 0.7 to $3.3 \mathrm{~h}$ ) and recreational screen time was $48 \mathrm{~min}$ higher ( -36 to $132 \mathrm{~min}$ ) during lockdown, compared to before lockdown. Discussion: While total screen time was markedly higher during lockdown, differences in physical activity and sleep seemed less pronounced than reported in other countries. The STAR24 holds promise as a single tool that can assess compliance with 24-h movement guidelines while providing clear illustrations of how populations are using their time and richer data to inform public health initiatives.

\section{Direct Observation of COVID-19 Transmission Behaviors and Physi- cal Activity in Public Open Spaces}

Richard Suminski Jr. ${ }^{1}$, Gregory Dominick ${ }^{1}$, Megan Heintzelman ${ }^{1}$

${ }^{1}$ University of Delaware

Introduction: Preventative behaviors (e.g. mask wearing) reduce COVID19 transmission; however, little is known about the practice of these behaviors during physical activity (PA). The purpose of the current study was to describe mask wearing and physical distancing behaviors in relation to PA performed in public open spaces using the direct observation method. Methods: A wearable video device was used to obtain videos along a 4.7 mile route that coursed through educational, retail/business, and residential areas. Videos were collected once/week during 6 weeks in the Fall and 6 weeks in the Spring between 11:15 a.m. and 12:19 p.m. Information of interest [PA type (walking, jogging, cycling), face masks presence, physical distance from others ( $<$ or $>6$ feet), and physical characteristic (e.g., sex/gender)] was manually extracted from videos by trained reviewers using comprehensive rules and computer manipulations (e.g., zooming). Results: A total of 1949 people were described. Most were $18-30$ years of age $(79.4 \%)$, white $(85.5 \%)$, female $(58.9 \%)$ and normal weight $(80.8 \%)$. Individuals engaged in PA were more compliant with mask wearing and physical distancing than people not engaged PA [80.5\% vs. $60.7 \%$ compliance; $\left.\chi^{2}(1,1882)=77.7 ; p<.001\right]$. Compliance did not differ between PA types. Among physically active individuals, compliance varied by minority categorization $[90.7 \%$ of minorities compliant vs. $78.0 \%$ of non-minorities; $\left.\chi^{2}(1,1299)=16.4 ; \mathrm{p}<.001\right]$ but not by $\mathrm{sex} /$ gender $(81.9 \%$ of males compliant vs. $79.2 \%$ of females) or weight status (79.5\% of normal weight compliant vs. $80.7 \%$ overweight/obese). Certain environments (e.g. crosswalks) were identified as "hot spots" where higher rates of adverse transmission behaviors occurred. Discussion: Direct observation was used to describe COVID-19 transmission behaviors as they relate to PA. Data obtained in this manner may be useful for assessing micro-scale phenomena for use in agent-based modeling and policy formation.

Feasibility and Effectiveness of an Online Exercise Group to Promote Physical Activity in Chronic Aphasia

\author{
Albert Mendoza $^{1}$, Jennifer Sherwood ${ }^{1}$, Michelle Gravier ${ }^{1}$
}

${ }^{1}$ California State University East Bay

Introduction: Aphasia is an acquired language disorder affecting $~ 2.5$ million people in the U.S. that has been shown to negatively impact quality of life (QoL) and physical activity (PA). Regular PA and decreased sedentary behaviors (SB) may slow physical decline and improve QoL in a typicallyaging population. However, limited research has been conducted examining the role PA may play in QoL for IWA. The present study aims to evaluate the feasibility and effectiveness of an aphasia-friendly exercise class on QoL, PA, and SB. Methods: Eleven participants (55\% female); age mean(SD): 62(14) years consented and were given QoL assessments at pre- and post-intervention. IWA enrolled in the exercise class were assigned to the INT group ( $=9)$, and those enrolled in other aphasiafocused groups were assigned to the $\mathrm{CON}$ group $(\mathrm{n}=2)$. Aphasia severity, as measured by the Quick Aphasia Battery overall score at pre-intervention ranged from 2.10 (severe) to 9.3 (very mild). 10 weeks of twice-weekly, 50-minute exercise sessions consisting of range of motion, aerobic, muscular endurance and strength were conducted via Zoom. Free-living, PA data was collected using activPAL 4+ (thigh-worn [on most active leg]) for 7-consecutive days at pre- and post-intervention. Valid wear days ( $\geq 4$ days with $\geq 10$ hrs. of waking wear) were examined. Paired-, and twosample t-tests were used to examine participant average change and mean group differences, respectively, significance set at $\mathrm{p}<0.05$. Results: Average attendance was high $(80 \%)$ and IWA with a range of severities were able to participate, supporting feasibility. The Burden of Stroke (BOSS) "positive emotions" subscore improved significantly for the INT but not the CON group $(\mathrm{p}=0.04)$. Standing time decreased significantly less for the INT than the CON group $(\mathrm{p}=0.02)$. Discussion: IWA may benefit from web-based, aphasia-friendly interventions aimed to improve QoL and increase PA, decrease SB. (Figure 58)

\section{Number of Daily Steps Among Finnish Children and Adolescents Was Lower During Covid-19-Lockdown in Spring 2020 Compared to Spring 2018}

Pauliina Husu ${ }^{1}$, Tommi Vasankari ${ }^{1}$, Anne-Mari Jussila ${ }^{1}$, Kari Tokola ${ }^{1}$, Henri Vähä-Ypyä ${ }^{1}$, Sami Kokko ${ }^{2}$, Harri Sievänen ${ }^{1}$

${ }^{1}$ The UKK Institute for Health Promotion Research, ${ }^{2}$ University of Jyväskylä, Faculty of Sport and Health Sciences

Introduction: Covid-2019 forced countries worldwide to use different social distancing policies. In Finland, the schools and many hobbies 
switched to distance learning (lockdown) for two months during spring 2020. Physical behavior was measured in a sample of children and adolescents during the lockdown and the results were compared to correspondingly measured data from the Finnish School-age Physical Activity (FSPA) 2018 study. Methods: During the lockdown, a sample of children and adolescents was recruited from two local areas and participants physical behavior was measured by a triaxial accelerometer for seven consecutive days. During waking hours, the accelerometer was worn at the right side of the hip and during sleeping hours at the nondominant wrist. The same method was used in the FSPA study, where a random sample of schools was drawn from the Statistics Finland Database. Children and adolescents aged 7-, 9-, 11-, 13- and 15-years were recruited via selected schools to complete a nationally representative sample. The Mann-Whitney U test was used to test differences in the mean numbers of daily and hourly steps among the similar-aged participants from the same two areas in spring $2020(\mathrm{n}=255)$ and spring $2018(\mathrm{n}=288)$. Results: The number of daily steps on weekdays was on average 8,383 in 2020 and $11,833$ in 2018 ( $\mathrm{p}<0.001)$. During the weekend, the corresponding step numbers were and 8,881 and 9,299 , respectively $(p=0.100)$. During the weekdays, children and adolescents accumulated on average 200-800 fewer steps per hour from 7 am to $2 \mathrm{pm}$ in 2020 than in 2018. During the evening hours on weekdays and the weekend, there were no systematic differences in hourly step numbers between 2020 and 2018. Discussion: Significantly lower step numbers during the school hours measured in 2020 may lead to decreased health and wellbeing among children and adolescents. Potential long-term adverse consequences of lockdown measures should be carefully scrutinized.

Objective Measurement of the Evolution of the Distribution of Physical Activity in Different Social Times During Confinement Against COVID-19: A Longitudinal Follow-Up of French Adolescents to Understand the Process of Resilience in Physical Activity

Thibaut Derigny ${ }^{1}$, François Potdevin ${ }^{1}$, Georges Baquet ${ }^{1}$, Joseph Gandrieau $^{1}$, Christophe Schnitzler ${ }^{1}$

${ }^{1}$ Université de Lille, Artois, Univ. Littoral Côte d'Opale

Introduction: The health crisis and resulting lockdowns are life events which disrupt physical activity (PA) lifestyles for adolescents, a population whose PA levels are already problematic. Whilst no other studies have used objective measurements to quantify the loss of PA in youth following lockdowns, this study seeks to examine how the lockdown impacted PA according to different social times throughout the day, using accelerometry. Methods: 43 French adolescents (age: $17 \pm 1.1$ y.o.) participated in a longitudinal study, which took place one week before lockdown (October 2020), and during the second week of lockdown (November 2020) using ActiGraph GT3X accelerometers. Respectively following the algorithms of Troiano 2007 and Freedson 1998, we determined the wearing periods and the PA cut points. At the same time, these adolescents completed a daily digital diary to reconstruct the ten daily social times: transport, home, school, physical education lesson, unsupervised leisure, supervised leisure, household chores, job, meals and rest, which were synchronized with the accelerometric data. Results: Firstly, the overall PA decreases significantly due to the reorganization of social times associated with PA: transport, physical education, supervised leisure. Secondly, we show a significant increase of unsupervised leisure. However, the latter is not able to compensate for the loss of the aforementioned social times. Finally, the loss in PA is correlated with the initial PA profile of the students. Discussion: We show that lockdown has reorganized the entire distribution of PA across social times, shifting the peak of physical activity from supervised leisure and PE to unsupervised leisure. Based on a temporal division we highlight which population has had its PA the most impacted and, more importantly, which social times are to be targeted in order to reduce this loss of PA.

Participants Experiences of a Six-Week Remote Sedentary Fractionation Digital eHealth Intervention With 24-Hour Accelerometery Monitoring During COVID-19

Aidan Buffey ${ }^{1}$, Brian Carson ${ }^{1}$, Alan Donnelly ${ }^{1}$

${ }^{1}$ University of Limerick

Introduction: Objective physical activity (PA) and sedentary behaviour (SB) data from adults during COVID-19 is limited, barriers include the distribution and cost of accelerometers. Accelerometers are not always suited to long-term monitoring, due to the battery life, storage capacity and participant compliance. The thigh-worn activPAL3M accelerometer allows remote data upload via USB-C and the participant can start a new recording. This study aimed to investigate the feasibility, acceptability and challenges of prolonged remote activPAL3M device wear. Methods: Twenty participants received a thigh worn activPAL3M to complete a six-week sedentary break fractionation intervention. The activPAL3M was worn six full days a week (24-hour protocol) for six weeks. On the seventh day (Sunday) participants remotely uploaded the weeks activPAL3M recorded data using 'PALtransfer' and restarted a recording for the coming week, simultaneously charging the device. Post study, participants completed an online survey [Qualtrics] with questions asking for their experiences of wearing the activPAL3M and of 'PALtransfer'. Results: Eight participants had no issues with wearing the activPAL3M for the duration of the study. Three participants were conscious of the device in the early weeks or 'during evenings' and when wearing 'tight clothes'. Six participants reported irritation from the Tegaderm used to secure the activPAL3M when worn 'long-term'. Ten participants found uploading the data via 'PALtransfer' 'easy' and 'fine'. Five participants reported 'difficulty' with the first upload 'whilst getting the hang'. Discussion: The activPAL3M and 'PALtransfer' software enabled participants to remotely upload objective accelerometer PA and SB data and begin new recordings allowing a single activPAL3M to be worn for six weeks. This development allows for longer monitoring periods without the exchange of devices, reducing the time burden of swapping devices to both the participant and researcher.

\section{Physical Activity Levels, Mental Health and Wellbeing in Children} and Young People in Wales During COVID-19

Liezel Hurter ${ }^{1}$, Melitta McNarry ${ }^{1}$, Denise Hill ${ }^{1}$, Gareth Stratton ${ }^{1}$, Kelly Mackintosh

${ }^{1}$ Swansea University

Introduction: The COVID-19 pandemic has caused unprecedented disruption to the lifestyles of children, with school closures resulting in the withdrawal of the primary source of structure and physical activity (PA). The aim of this study was to determine the PA levels, mental health and wellbeing of children and young people in Wales during lockdown. Methods: 1,708 participants ( 8 - 18 years; 858 girls) completed an online survey measuring PA levels, mental health and wellbeing, with a subsample of 800 , stratified by age, sex and socio-economic status, wearing an Axivity AX3 accelerometer on their non-dominant wrist for seven days. The survey included three validated questionnaires: the Stirling Children's Wellbeing Scale, the Good Childhood Index, and the Physical Activity Questionnaire for Older Children (PAQ-C) or Adolescents (PAQ-A), dependent on age. Raw accelerometer data were processed in R-package GGIR and analysis of covariance were used to compare between-group variables. Results: PA levels were low, with mean PA scores from PAQ-C and PAQ-A of $2.2 \pm 0.7$ and $1.9 \pm 0.6$. Accelerometer results confirmed this, 
with only $13 \%$ of participants meeting UK government guidelines for PA, and accumulating, on average, $848 \pm 204$ mins of sedentary time per day. PA levels $(F=9.4)$, wellbeing $(F=17.6)$ and life-satisfaction scores $(\mathrm{F}=41.1)$ declined significantly with age (all $\mathrm{p}<0.001)$. No significant sex differences were found in PA, irrespective of self-report $(p=0.57)$ or device-based $(\mathrm{p}=0.33)$ measures. However, boys scored significantly higher for wellbeing $(\mathrm{F}=25.9 ; \mathrm{p}<0.001)$ and life-satisfaction $(\mathrm{F}=10.7 ; \mathrm{p}=0.001)$. Discussion: The national lockdown has resulted in children spending most of their time being sedentary. Converse to previous research, there were no significant sex differences, suggesting that boys' PA levels were more negatively affected than girls'. The PA levels and wellbeing of secondary school children are specifically of concern.

\section{Preliminary Evaluation of a Remote Digital Ehealth Sedentary Time Fractionation Intervention for Older Adult Home-Based Workers During COVID-19}

\author{
Aidan Buffey ${ }^{1}$, Brian Carson ${ }^{1}$, Alan Donnelly ${ }^{1}$ \\ ${ }^{1}$ University of Limerick
}

Introduction: Prolonged sitting has been detrimentally associated with measures of cardiometabolic health. Individuals working from home (WFH) due to COVID-19 guidance may increase their sedentary time due to everyday movement being restricted, increased screen time to stay connected and home environment. This study aimed to objectively examine the physical activity (PA) and sedentary behaviour (SB) patterns of adults WFH and reduce bouts of $\mathrm{SB}>60$ mins, via a remote eHealth intervention designed to break sitting during working hours every 45 minutes with five minutes of light-intensity walking. Methods: Twentytwo participants (>30 years) were recruited from the University of Limerick. Participants fitted a thigh worn activPAL3M accelerometer, worn continuously (24-hour protocol) six days a week for six weeks. Utilising an AB design, Week 1 to Week 3 was the baseline period, where participants continued their habitual PA and SB. Week 4 to Week 6 was the intervention period, where participants followed the eHealth intervention. The eHealth intervention used a desktop application [BreakTimer] which appeared on the participants' workstation screen every 45 minutes prompting participants to complete five minutes of walking, breaking their sitting. Results: Paired-sample t-tests revealed a significant reduction in the number of sedentary bouts $>60$ mins $(-14.55 \%, \mathrm{p}=0.047),>90$ mins $(-27.63 \%, \mathrm{p}=0.017)$ during working hours and the time accumulated in bouts of sedentary behaviour $>60$ mins $(-18.01 \%, p=0.003)$ and $>90$ mins $(-27.34 \%, \mathrm{p}=0.011)$ during the intervention compared with control periods (See Figure 59). Discussion: An eHealth intervention designed to prompt breaks from sitting every 45 minutes with five minutes of walking during working hours significantly reduced the number of sedentary bouts $>60$ and $>90$ mins and reduced the accumulated time spent sedentary in bouts $>60$ and $>90$ mins. The findings suggest a digital prompt reduced prolonged bouts of sitting in adults WFH.

Project COPE: An Investigation of Daily Experiences of Stress, Physical Activity, and Sleep During the COVID-19 Pandemic

Rachel Lyons ${ }^{1}$, Kristina Hasanaj ${ }^{1}$, Kasondra McCracken ${ }^{1}$, Cheryl Der Ananian $^{1}$, Matthew Buman ${ }^{1}$

${ }^{1}$ Arizona State University

Introduction: Prolonged COVID-19 pandemic stress is a major public health concern linked to recent increases in depression, anxiety, and PTSD. Strategies to lower stress, particularly in vulnerable sub-groups, are needed. Adults forced to work from home due to COVID-19 might be among the most stressed, as they lack important resources and must balance work and family roles within the same geophysical space.
Moderate-to-vigorous physical activity (MVPA) and sleep duration/quality are potent strategies for lowering stress; yet their role during the COVID-19 pandemic are not known. This study examined time-varying associations of MVPA and sleep with momentary perceived stress during the COVID-19 pandemic to discern their role in decreasing stress. Methods: Thirty remote-working adults ( $86.7 \%$ women; age 37.5 $[ \pm 10.4]$ years) volunteered for the 14-day study. Wrist-worn GENEActiv accelerometers continuously measured MVPA and sleep, and four Ecological Momentary Assessments (EMAs) per day assessed stress. The impact of between- and within-person variations in MVPA, sleep quality rating (SQR), total sleep time (TST), and sleep efficiency on momentary stress were analyzed via multilevel models. Results: Between-person models revealed a significant negative effect of SQR on perceived stress (b [se] $=-0.65$ [0.30], $\mathrm{p}=.04$ ). MVPA, TST, and sleep efficiency were not significant between-person predictors of stress. Within-person models indicated that higher than normal MVPA (b [se] $=-0.005[<0.01]$, $\mathrm{p}=0.02), \mathrm{SQR}(\mathrm{b}[\mathrm{se}]=-0.28$ [0.07], $\mathrm{p}<.001)$, TST $(\mathrm{b}$ [se $]=-0.01$, $<0.001, \mathrm{p}=0.01$ ), and sleep efficiency ( $\mathrm{b}$ [se] $=-0.52$ [0.24], $\mathrm{p}=.03$ ) were all associated with significant decreases in momentary stress. Discussion: Daily fluctuations in MVPA and sleep correlate more strongly with momentary stress than do overall patterns of these behaviors. Leveraging slight improvements in habitual MVPA and sleep is an attainable strategy for mitigating stress during the COVID-19 pandemic.

\section{Quantitative Physical Activity Assessment in Children During a COVID-19 Stay-At-Home Order}

Kirsten Tulchin-Francis ${ }^{1}$, Wilshaw Stevens, $\mathrm{Jr}^{2}$, Cristina Lopez ${ }^{2}$, Heather Roberts $^{3}$

${ }^{1}$ Scottish Rite for Children/UT Southwestern, ${ }^{2}$ Scottish Rite for Children, ${ }^{3}$ Scottish Rite for Children/Texas Woman's University

Introduction: Physical activity (PA) is vital for physical fitness, mental health and social development. US guidelines recommend children 517years achieve 60min of moderate-to-vigorous PA (MVPA) daily, or approximately 12,000 steps. COVID-19 led to social distancing restrictions, including closure of schools and playgrounds and cancellation of organized sports. The aim of this study was to assess quantitative PA during COVID-19 Stay-at-Home(CSAH) orders in typically developing (TD) youth. Methods: TD children (5-10yrs) living in a single US metroplex were given a StepWatch Activity Monitor (SAM) to wear for 5 days ( 3 weekdays/2 weekend) during CSAH in April 2020. Strides were doubled for total steps. Retrospective data from age-matched children (same metroplex) pre-pandemic were used as a historical (HIS) control. Non-parametric tests $(\alpha<.05)$ were used to determine differences between groups and weekdays/weekend days. Post-hoc power analysis required 14 subjects/group. Results: There was no difference in age $(\mathrm{CSAH}=33,15 \mathrm{~F}, 7.4 \pm 0.3 \mathrm{yrs} ; \mathrm{HIS}=26,14 \mathrm{~F}, 8.0 \pm 0.2 \mathrm{yrs}, \mathrm{p}=.09)$. CSAH had $77 \mathrm{~min}$ less active time $(\mathrm{p}=.001)$ and 3,737 less steps/day ( $\mathrm{p}<.001$ ) compared to HIS. Only $36 \%$ of CSAH averaged 12,000 steps/ day (HIS:85\%); this dropped to $27 \%$ on weekdays (HIS:92\%). HIS had reduced weekend steps compared to weekdays $(\mathrm{p}=.04)$, with no difference in CSAH $(\mathrm{p}=.58)$. Discussion: Children during COVID-19 SAH orders had significantly reduced PA compared to a pre-pandemic data. 12,000 steps/day has been shown to be an accurate proxy to reaching minimal MVPA recommendations. School closure and reduced peer play appear to significantly impact child PA as pre-pandemic differences between weekdays/weekends were not seen during CSAH. Schools should assure quality physical education remains an essential part of in-person or virtual instruction. Parents should be educated on the importance of PA outside of the classroom and offered suggestions for home, neighborhood and community options. (Figure 60) 
Smartphone-Delivered, Home-Based Gait Training for Persons With Parkinson's Disease: Feasibility of a Tele-Rehabilitation Program

Mattia Corzani ${ }^{1}$, Giovanna Lopane ${ }^{2}$, Valeria Petrone ${ }^{2}$, Fabio La Porta ${ }^{2}$, Marina Brozgol $^{3}$, Nir Giladi ${ }^{3}$, Pablo Thumm ${ }^{3}$, Jeffrey Hausdorff ${ }^{3}$, Lorenzo Chiari $^{4}$, Luca Palmerini ${ }^{4}$

${ }^{1}$ University of Bologna, ${ }^{2}$ IRCCS Istituto delle Scienze Neurologiche di Bologna, ${ }^{3}$ Tel-Aviv Sourasky Medical Center, ${ }^{4}$ Health Sciences and Technologies - Interdepartmental Center for Industrial Research (CIRI-SDV)

Introduction: Telerehabilitation allows patients to utilize rehabilitation services at home. The novel telerehabilitation system tested in this study is an innovative wearable device (smartphone +2 IMUs on the shoes, Gait Tutor, GT) that was developed to provide gait training to people with Parkinson's Disease (PD) in the real-world, outside of specialized centers. GT provides real-time feedback to users based on IMUs placed on the shoes. In a previous project, $\mathrm{CuPiD}, 20$ patients with $\mathrm{PD}$ used the system for 6 weeks without adverse side effects. Based on these initial results, we aim to assess the feasibility and potential efficacy of a GT training program of 9-months. Methods: Twenty patients with PD are undertaking gait training in the ON therapy condition for 30 minutes, 3 times/week for 9 months using the GT. The system provides positive and corrective biofeedback (BF) on selected gait parameters (e.g., step length). Here we report the results on the first subject, P1 (71 yrs, F, Hoehn and Yahr: II, disease duration $=8 \mathrm{yrs}$ ). Results: $\mathrm{P} 1$ performed 111 trials out of the 117 planned in 9-months. During lockdown, P1 performed 13 trials/month vs 11 trials/month in the routine periods. Figure $61 \mathrm{~A}$ shows the trend of the main gait outcomes achieved in each trial. Figure 61B reports the trend of the percentage of praising and corrective $\mathrm{BF}$ received in each trial. Measures of progression are shown in Figure 61C. Discussion: Wearable systems for self-administered gait training can provide a way to ecologically improve and prolong the effects of exercise in PD subjects, potentially reducing costs. The compliance of $\mathrm{P} 1$ with the training program was very high. The clinical data suggest an improvement in UPDRS-III and a benefit on postural behavior. During lockdown, P1 performed 15\% more trials than the routine periods. The use of GT at home during the 9months allowed for maintaining physical activity, possibly mitigating the detrimental effects of the COVID lockdown.

\section{The Effect of COVID-19 Restrictions on Level of Physical Activity and Health in Older Home-Dwelling Adults in Norway}

Arnhild Nygård ${ }^{1}$, Kristin Taraldsen ${ }^{1}$, Randi Granbo ${ }^{1}$, Geir Selbæk ${ }^{2}$, Jorunn Helbostad ${ }^{1}$

${ }^{1}$ Norwegian University of Science and Technology, ${ }^{2}$ Oslo University Hospital

Introduction: The spread of the coronavirus spring 2020 led to lockdown of indoor physical activity and exercise groups. The aim of this study was to investigate how physical activity, general health and mental health in community-dwelling older adults was affected by the COVID-19 restrictions in Norway. Methods: This study used a retrospective design. Inclusion criteria were being 65 years and older and living at home. Invitation was sent via Facebook or by newsletters. Participants completed a questionnaire either digitally or as a paper version. The questionnaire included questions on physical exercise (5-point Likert scale), general health (5-point Likert scale) and mental health (CONOR-MHI) before (13th of March) and during lockdown (June-August 2020). Results: The 565 participants had mean age of $74( \pm 5.3$, range: $65-95)$ years, $60.4 \%$ were female, almost $60 \%$ had a university degree, $84 \%$ reported being physically active more than once per week, and about $20 \%$ reported a fall the previous 12 months. Wilcoxon signed-rank test showed that the corona lockdown significantly reduced both activity level, general health and mental health (see Figure 62). Compared with those who exercised individually before the 13th March, the odds ratio for being less active for those attending group exercise were 2.91 (95\% CI $=1.97-4.29$, $\mathrm{p}<0.001$ ). Odds of being less active during lockdown increased with a decrease in general health status. Those who were less active during lockdown experienced worse health than those who maintained their activity level, Odds ratio $27.29(95 \% \mathrm{CI}=10.68-69.72, \mathrm{p}<0.001)$ for general health and 3.08 (95\% CI $=2.10-4.51, \mathrm{p}<0.001)$ for mental health, respectively. There were no significant higher or lower odds for gender or type of exercise. Discussion: We included a relatively highly educated and active group of older participants in this study, but the COVID-19 restrictions still affected both level of activity and general and mental health in a negative way.

Using the Fitbit to Understand the Long-Term Impact of COVID-19 Lockdown on Activity Levels Amongst Intervention Trial Participants With Type 2 Diabetes

Agus Salim ${ }^{1}$, Genevieve Healy ${ }^{2}$, Alison Carver $^{3}$, Neville Owen ${ }^{1}$, David Dunstan

${ }^{1}$ Baker Heart and Diabetes Institute, ${ }^{2}$ The University of Queensland, ${ }^{3}$ Australian Catholic University

Introduction: Lockdown restrictions have reduced COVID-19 community transmissions; however, they may pose challenges for non-communicable disease management. A 112-day hard lockdown in Victoria, Australia (commencing March 23, 2020), which affected an intervention trial of reducing and breaking up sitting time in desk workers with type 2 diabetes, provided the opportunity to compare long term activity levels during and preceding lockdown using a provided fitbit Inspire HR. Methods: Participants wore a fitbit continuously during all waking hours; for this analysis, data was collected between August 2019 and September 2020. Step and heart rate data were collected at 1-minute intervals. Energy expenditure (METs) was automatically estimated from device-measured heart rate and categorized as inactive, light, moderate, and vigorous activity for each minute. Linear regression models were used to compare post-to-pre lockdown behaviours, and were adjusted for individual diurnal activity cycle, and seasonal effect. Results: A total of 2309 wear days were recorded across 11 participants with a median of 194 days (min: 160; max: 374) per participant. There was significant heterogeneity between participants and in the impact of lockdown on their activity levels, with an overall decrease in the post-to-pre rate of steps (RR: 0.97; 95\% CI: 0.96, 0.98) and METs (RR: 0.99; 95\%CI: 0.99, 0.99); rate of time spent inactive increased (RR: 1.03; 95\%CI: 1.03, 1.04), whilst light (RR: 0.97; 95\%CI: 0.97, 0.98) and moderate (RR: 0.95; 95\%CI: 0.94, 0.97) activity decreased. Discussion: For participants with type 2 diabetes enrolled in a sittingreduction intervention trial, the lockdown resulted in an overall decrease of step count, energy expenditure, light and moderate activity, and an increase of inactivity. Data from the wrist-worn Fitbit consumer device can provide interpretable longer-term activity data, which now requires corroboration using concurrent data from research grade devices. (Figure 63)

\section{Measurement \& Analysis: Future Perspectives}

A Systematic Review and Repository of Novel Methods for Estimating Physical Activity and Energy Expenditure From Accelerometer Data

Kimberly Clevenger ${ }^{1}$, Andrew Kaplan ${ }^{2}$, Cailyn Van Camp ${ }^{3}$, Alexander Montoye $^{4}$, Scott Strath ${ }^{5}$, Karin Pfeiffer ${ }^{3}$

${ }^{1}$ National Cancer Institute, ${ }^{2}$ Indiana University School of Medicine, ${ }^{3}$ Michigan State University, ${ }^{4}$ Alma College, ${ }^{5}$ University of WisconsinMilwaukee 
Introduction: Raw acceleration data and "novel" analytic approaches like machine learning for physical activity measurement will not be widely used if they are not accessible to other researchers. This systematic review characterizes the accessibility and use of novel analytic techniques for classifying energy expenditure and/or physical activity intensity using raw or count-based accelerometer data. We establish an online repository for these methods to increase accessibility and use. Methods: Three databases were searched for relevant articles published between January 2000 and February 2021. Whether the authors provided access to the model (e.g., by request, sample code) or not was recorded. To quantify use of each model, the number of articles using each method was coded from a list of citing articles compiled from Google Scholar. Available code, models, sample data, and instructions were compiled and added to the repository. Results: Studies $(\mathrm{N}=162)$ included adults $(\mathrm{n}=141)$, and/or children $(\mathrm{n}=34)$. Half of articles $(n=107)$ did not provide access to their model or included partial access $(n=19)$, while 4 indicated it could be provided upon request. Authors who provided access to the model $(\mathrm{n}=55)$ included equations, cut-points, or decision trees in the paper $(n=43)$ or provided access to code $(n=13)$. Use of models ranged from 0 to 24.75 uses/year (average 0.62), with 91 models that have not been used yet. Approximately half of uses occurred in a free-living setting (49\%) and/ or by the same authors (42\%). Models from fifty-five articles are now included in the repository. Discussion: The proliferation of approaches for analyzing accelerometer data outpaces the use of these models in practice which is unsurprising as less than half of the developed models are made accessible for use by other researchers. The repository should facilitate accessibility and use of novel methods for analyzing accelerometer data, improving data harmonization and consistency across studies. (Figure 64)

\section{Assessment of Cardiovascular Demand Using Wrist-Based Wearables}

Kevin Abbruzzese ${ }^{1}$, Vincent Alipit ${ }^{1}$, Sally LiArno ${ }^{1}$

${ }^{1}$ Stryker Orthopaedics

Introduction: Wearable devices provide the ability to monitor physiological signals in real time. Heart rate can provide insight into cardiovascular performance. Cardiovascular demand (CVD) is a multivariate biometric feature that examines heart rate response based on duration, intensity, and stress of activity. Several wrist-based wearables were assessed to determine overall cardiovascular demand during exercise. Methods: Several watch-based wearable sensors were assessed by a single individual. WHOOP, Garmin VIVOSMART® 4 and Fitbit Inspire were worn to monitor CVD during one hour of high-intensity activity. Each device provides estimations of cardiovascular performance. WHOOP Strain, Garmin Body Battery, Garmin Stress and Fitbit with Personal Activity Intelligence (PAI) were documented during activity. Results: CVD was assessed based on two factors: stress and exertion. An Anderson Darling test was performed to assess normality for both groups $(\mathrm{p}>0.05)$ and an ANOVA test was used to determine statistical differences. No significant differences were observed in the stress group between WHOOP Strain and Garmin Stress $(\mathrm{p}=0.790)$. No significant differences were observed for exertion factor between Fitbit with PAI and Garmin Body Battery. ( $p=0.145)$. Discussion: Cardiovascular demand represents the physiological impact from activities as an estimation of user exertion and physiological function. Intensity and duration can influence CVD scores. User exertion can be represented by PAI or Garmin Body Battery. Autonomic physiological responses can be estimated by heart rate variability and indicate stress. These CVD metrics can provide insight into stress and exertion and impact on cardiovascular function. (Figure 65)

\section{Biometric Accuracy From Wrist-Based Wearables}

Kevin Abbruzzese ${ }^{1}$, Vincent Alipit ${ }^{1}$, Sally LiArno ${ }^{1}$

${ }^{1}$ Stryker Orthopaedics

Introduction: Wearable biosensor technologies provide the ability to monitor biometrics in real time. Physiological signals like heart rate (HR) can be captured from wrist-based wearables. The accuracy of the biometric data can be variable based on the device and intensity of the activity. Several wrist-based photoplethysmography (PPG) wearables were assessed during intense activities and compared to the gold standard Polar H10 chest strap control. Methods: A within-subject assessment $(\mathrm{N}=1)$ was designed to compare several watch-based wearable sensors over five sessions. Fitbit Inspire, Garmin VIVOSMART® 4, Polar H10 and WHOOP were simultaneously worn to monitor HR during one hour of high-intensity activity. Wrist-based wearables were worn on both dominant and non-dominant hand. Average heart rate data was assessed and compared to Polar H10 chest strap data, Figure 66. Results: An AndersonDarling test was performed to assess normality $(\mathrm{p}=0.591)$. ANOVA statistical test was performed to assess significance between groups and significance was observed between devices $(\mathrm{p}<0.001 \mathrm{~A}$ Tukey post-hoc analysis determined that the HR reported by the WHOOP device was significantly lower than those reported by Fitbit and Garmin. Reported HR for wrist-based wearables were not statistically significant from HR values measured by Polar H10 chest strap. Discussion: The demand for mobile health and wellness monitoring continues to increase. While no significant differences were observed in HR between devices and control, WHOOP reported significantly lower HR values compared to the other wrist-based wearables. This difference in HR suggests that multivariate factors dependent on HR magnitude, like stress and energy expenditure could also result in differences.

\section{Canadian Health Measures Survey Cycle 7: Changes to the Movement Behaviour Measurement Protocol}

Nicholas Kuzik $^{1}$, Janine Clarke ${ }^{1}$, Rachel Colley ${ }^{1}$, Thomas Ferrao ${ }^{1}$, Aidan Gribbon $^{1}$, Michelle Guerrero ${ }^{1}$, Nadjet Oulhaci ${ }^{1}$, Jennifer Servais ${ }^{1}$, Mélie St-Laurent ${ }^{1}$, Mark Tremblay ${ }^{2}$

${ }^{1}$ Statistics Canada, ${ }^{2}$ Children's Hospital of Eastern Ontario Research Institute

Introduction: Statistic Canada's Canadian Health Measures Survey (CHMS) is the main surveillance tool for examining movement behaviours (i.e., sleep, sedentary behaviours, and physical activity) in Canada. Since 2007, the CHMS has used the Actical accelerometer (right hip, waking day wear protocol). However, in the next cycle of data collection (estimated: Jan 2022-Dec 2023), the CHMS will use the ActiGraph wGT3X-BT (right hip, 24-hour wear protocol). To compare previous estimates of movement behaviours using Actical data with the forthcoming national estimates using ActiGraph data, a pilot study is underway to facilitate this transition. Methods: Participants $(n=150)$ aged 3-79 will be recruited to simultaneously wear ActiGraph and Actical accelerometers 24 hours/day for 7 days. Previous CHMS data will be used to compare waking and 24-hour wear protocol data. Results: Techniques will be created to translate 24-hour wear time outputs from Actical to ActiGraph. Further, techniques will be created to translate waking day Actical outputs to 24-hour ActiGraph and Actical outputs. Analyses will separate participants into several age groups. Discussion: This research will allow Canada's movement behaviour surveillance system to determine trends in movement behaviours across past and future cycles of data collection. Establishing continuity in outputs is imperative to understand the associations between movement behaviours with a variety of health outcomes in a nationally representative sample, which can subsequently steer public health initiatives and policy. 
While some data collection decisions are fixed (e.g., accelerometer brand), future data processing decisions need to be considered moving forward. For instance, consensus will be needed for the epoch length, valid wear time definitions, and movement behaviour classifications. Expert feedback will be solicited to strengthen data processing decisions, and techniques used to translate between wear protocols and devices.

\section{Changes in Pre-Frontal Cortex Oxygenation During Linear and Curvilinear Walking Trajectories: A Combined fNIRS and IMUs Study}

Valeria Belluscio ${ }^{1}$, Gabriele Casti $^{1}$, Marco Ferrari $^{2}$, Valentina Quaresima ${ }^{2}$, Jorn Horschig $^{3}$, Maria Sofia Sappia ${ }^{3}$, Giuseppe Vannozzi ${ }^{1}$

${ }^{1}$ University of Rome Foro Italico, ${ }^{2}$ University of L'Aquila, ${ }^{3}$ Artinis Medical Systems

Introduction: Functional near infrared spectroscopy (fNIRS) measures brain activity. Increased oxygenated hemoglobin $(\mathrm{O} 2 \mathrm{Hb})$ level in the prefrontal cortex (PFC) was observed during linear walking, particularly when there is a high attention demand on the task, like dual-task (DT) paradigms. While linear walking is an automatic task, curvilinear walking involves cognitive to motor adaptations, but despite its ecological value, no studies investigated PFC during this task. Therefore, PFC activity was assessed during linear and curvilinear trajectories, in single-task (ST) and DT. Methods: Twenty adults $(10 \mathrm{M}, 28.45 \pm 5.14 \mathrm{yrs})$ performed 3 linear and 3 curvilinear walking trials of 30 s each, the latter performed using the Figure-of-8-Walking-Test (F8WT), in both directions, in ST and DT using the "Serial 7s" test. PFC activity was measured using a $20+8$ channels fNIRS device (Brite 23,10 Hz, Artinis, NL), for both right and left hemispheres: each trial was divided into 5 s-time windows to observe eventual $\mathrm{O} 2 \mathrm{Hb}$ changes over time. Spatio-temporal and gait quality parameters were also quantified using 4 wearable inertial sensors (Opal, $128 \mathrm{~Hz}$, APDM, USA). Results: Increased $\mathrm{O} 2 \mathrm{Hb}$ was observed in both walking modalities in the first $5 \mathrm{~s}$ time-window of each trial, with the lowest values displayed at the end of the task (Figure 1, left hemisphere). These differences are evident during the F8WT in both directions and especially in the DT condition (panels d-f). Figure 67. Median O2Hb and IQR are displayed. Horizontal lines with asterisks $(*)$ indicate statistically significant differences among time-windows Results about motor performance confirmed the existing literature, with reduced walking speed, stride frequency, dynamic stability and body symmetry. Discussion: Curved walking in DT requires highest PFC activation compared to linear walking. Being more representative of real-life situations, curved walking has the potential to reveal crucial information about people's balance.

\section{Cross-Sectional Associations Between 24-hr Activity Behaviours and Cardiometabolic Health in Adolescents: A Compositional Data Analysis}

Leonard Browne $^{1}$, Kieran Dowd ${ }^{2}$, Ciarán McDonncha ${ }^{1}$, Brian Carson ${ }^{1}$, Helen Purtill ${ }^{1}$, Ailish Hannigan ${ }^{1}$, Matthew Herring ${ }^{1}$, Eibhlis O Connor ${ }^{1}$, Clodagh O' Gorman ${ }^{1}$, Alan Donnelly ${ }^{1}$

${ }^{1}$ University of Limerick, ${ }^{2}$ Athlone Institute of Technology

Introduction: By employing a compositional data analysis (CoDA), this study examined the relative associations between adolescent 24-hr activity behaviours and indicators of cardiometabolic health. It also investigated predicted differences in cardiometabolic health when time was reallocated between activity behaviours. Methods: Upon ethics committee approval, participants $(\mathrm{n}=222 ; 16.3$ years $(0.92) ; 49 \%$ Male) recruited from eight schools in the mid-west region of Ireland provided measures of body composition, cardiorespiratory fitness (CRF), grip-strength, blood pressure (BP); blood lipid and markers of glucose control. Objective measures of sleep, SedT, standing time, light intensity physical activity (LIPA) and moderate-to-vigorous physical activity (MVPA) were obtained from the activPAL 3 micro. Compositional linear regression models were used to examine the relationship between the aforementioned activity behaviours and cardiometabolic health indicators while controlling for age, sex and school location. Results: LIPA (relative to the remaining behaviours) was associated with the sum-of-skinfold thickness ( $\hat{a}=-13.9 ; \mathrm{p}<0.05)$, CRF $(\hat{\mathrm{a}}=3.9 ; \mathrm{p}<0.05)$ and diastolic BP $(\hat{\mathrm{a}}=-8.3 ; \mathrm{p}<0.05)$. Relative to the remaining behaviours SedT and standing time were associated with lower grip-strength ( $\hat{a}=-5.4 ; \mathrm{p}<0.05)$ and LDL-Cholesterol $(\hat{a}=-0.38$; $\mathrm{p}<0.05)$ respectively. MVPA was associated with HDL-Cholesterol $(\hat{\mathrm{a}}=0.7 ; \mathrm{p}<0.05)$ and triglycerides $(\hat{\mathrm{a}}=-0.1 ; \mathrm{p}<0.05)$. Reallocating 30 minutes from sleep, SedT or standing time to LIPA or MVPA, was associated with significant improvements in cardiometabolic health indicators (Figure 68).Discussion: The findings of the present study reinforce the health benefits associated with MVPA but also provide evidence to support recommendations to increase LIPA and standing time through decreasing sitting time to improve cardiometabolic health in adolescents.

\section{Energy Expenditure Estimates From Wrist-Based Wearables}

Kevin Abbruzzese ${ }^{1}$, Andre Freligh ${ }^{1}$, Vincent Alipit ${ }^{1}$, Sally LiArno ${ }^{1}$

${ }^{1}$ Stryker Orthopaedics

Introduction: The demand for mobile-health and wellness monitoring continues to increase. Physiological signals and energy expenditure estimates can be obtained from wrist-based wearables. The overall energy expenditure of an individual is dependent on time and intensity of an activity. Several wrist-based wearables were assessed during intense activities and compared to the gold standard Polar H10 wearable chest strap. Caloric energy expenditure was assessed between devices. Methods: A within-subject assessment $(\mathrm{N}=1)$ was designed to compare several watch-based wearable sensors over five sessions. Fitbit Inspire, Garmin VIVOSMART® 4, Polar H10 and WHOOP were simultaneously worn to monitor energy expenditure during one hour of high-intensity activity. Wrist-based wearables were worn on both the dominant and non-dominant hand. Caloric energy expenditure was assessed and compared to Polar H10 chest strap, Figure 69. Results: An Anderson Darling test was performed to assess normality $(\mathrm{p}>0.05)$. ANOVA statistical test was performed to assess significance in energy expenditure between groups, and significance was observed between devices $(p=0.001)$. A Tukey post-hoc analysis determined that caloric expenditure reported by the WHOOP device was significantly lower than those reported by the Fitbit, Garmin and Polar H10 devices. Discussion: Wearable devices provide the ability to capture activity and estimate individual output. Multivariate-dependent factors like energy expenditure are influenced by accuracy of heart rate data.

\section{Feasibility of Long-Term Heart Rate Monitoring Compared to Accel- erometry in Older Adults}

Laura Karavirta ${ }^{1}$, Jukka Lipponen ${ }^{2}$, Timo Rantalainen ${ }^{1}$, Erja Portegijs ${ }^{1}$, Taina Rantanen ${ }^{1}$

${ }^{1}$ University of Jyväskylä, ${ }^{2}$ University of Eastern Finland

Introduction: Heart rate monitoring can advance the assessment of physical activity intensity compared to accelerometry alone. However, medication and arrhythmias are common especially in older age and question the added value of the laborious heart rate monitoring. We investigated the exclusion rate in long-term heart rate monitoring in comparison to accelerometry, and physical activity in those included in and excluded from the heart rate analyses. Methods: Out of the population-based sample of 910 (75, 80 and 85 year old) adults who participated in the laboratory measurements, 495 consented to wear a thigh-mounted accelerometer 
(sampled at $100 \mathrm{~Hz}$ ) for a week. A single-channel ECG device was additionally offered to participants without a pacemaker (Figure 70). Daily mean acceleration and moderate-to-vigorous physical activity (MVPA) were computed. Walking speed and resting heart rate in supine position were measured in the laboratory. Medication was self-reported. Results: Of the 438 participants with $\geq 3$ days of heart rate data, $46.6 \%$ reported to use medication in at least one of the following groups: antiarrhythmic medications $(1.6 \%)$, beta blockers $(36.1 \%)$ or calcium channel blockers $(21.2 \%)$. The participants with valid heart rate and accelerometry data did not differ from those who had accelerometry only in terms of age, sex, or resting heart rate, but had higher walking speed $(1.2(0.2) \mathrm{m} / \mathrm{s}$ vs. $1.1(0.2) \mathrm{m} / \mathrm{s}$, $\mathrm{p}<0.001)$, daily average acceleration (26.0 (8.9) mg vs. 22.5 (7.4) mg, $\mathrm{p}<0.001$ ) and MVPA per week (253 (179) min vs. 186 (141) min, $\mathrm{p}<0.001)$. Discussion: The present results indicate potential selection bias in long-term heart rate monitoring by unintentionally excluding older people who are less physically active. The high exclusion rate is mainly due to the high proportion of older adults using medication known to affect heart rate. Loosening the inclusion criteria and simultaneous development of new validated methods regardless of medication are warranted.

\section{Longitudinal Associations Between Physical Activity Fragmentation Indices and Physical Function in Older Adults}

\section{Jason Wilson ${ }^{1}$, Mathias Skjødt ${ }^{2}$, Paolo Caserotti ${ }^{2}$, Mark Tully ${ }^{1}$}

${ }^{1}$ Ulster University, ${ }^{2}$ University of Southern Denmark

Introduction: Research using composite measures of physical activity variables describing the diversity of bout lengths, such as fragmentation indices, have shown higher physical activity fragmentation to be crosssectionally associated with lower physical function. However, few studies have prospectively investigated this association. This study explored the longitudinal associations between physical activity fragmentation and physical function in community-dwelling older adults. Methods: Six hundred and ninety older adults ( $\geq 65$ years old) recruited during the SITLESS project provided complete data at baseline and 22 months follow-up. Fragmentation indices were calculated as a reciprocal of the average physical activity ( $\geq 100$ counts per minute) bout duration measured using ActiGraph wGT3X+ accelerometers. Physical function was measured using the two-minute walk test (2MWT). Two minimum bout lengths were utilised to calculate fragmentation indices: $\geq 120$-seconds and $\geq 300$-seconds. Multiple linear regression analyses, after adjusting for relevant covariates, were used to assess longitudinal associations. Results: After adjustment for relevant covariates (Figure 71), including 2MWT distance at baseline, changes in physical activity fragmentation index using $\geq 300$-second bouts were associated with changes in 2MWT distance $(B=-0.09 ; \mathrm{p}=0.013)$. No significant association between physical activity fragmentation index using $\geq 120$-second bouts and $2 \mathrm{MWT}$ distance was found. Discussion: Using $\mathrm{a} \geq 300$-second minimum bout length, change to a more fragmented physical activity pattern appeared to be associated with reduced physical function in community-dwelling older adults. This study provides further evidence of the potential for physical activity fragmentation indices to be useful additional measures for researchers and suggests addressing how activity is accumulated may be a useful target for interventions specifically in older adults.

\section{Sleep and Exercise Among Physicians: Does One Affect the Other?}

Mary Hidde $^{1}$, Emily Williams ${ }^{1}$, Kate Lyden ${ }^{2}$, Julia Sharp ${ }^{1}$, Heather Leach ${ }^{1}$ ${ }^{1}$ Colorado State University, ${ }^{2}$ KAL Research \& Consulting, LLC

Introduction: Physicians have demanding jobs which may impact sleep and exercise habits. It is unclear whether a causal relationship exists between the two behaviors (i.e., does nighttime sleep impact exercise the next day?). The aims of this study were to evaluate (1) exercise and the following nights' sleep, and (2) sleep and the following day's exercise. Methods: Cardiologists $(n=12)$ and oncologists $(n=6)$ wore an activPAL accelerometer on the right thigh for 7-days, 24-hours per day to measure exercise (walking-related behaviors with a sustained cadence of $>75$ for $\geq 1$ minute) and sleep (non-upright events lasting $>1$ hour). In order to allow for common sleep interruptions (i.e., bathroom breaks, wake after sleep onset), events are expanded to adjacent non-upright events $>1$ hour. Participants with $\geq 4$ valid days ( $\geq 10$ hours of wear time and day/night pairs) were included in analyses. To examine associations between daily exercise and sleep, a linear mixed effects model was used, with participant as a random effect and specialty as a fixed effect $(\mathrm{p}<.05)$. Results: A total of 128 days of data were included, and participants spent an average of $17.9 \pm 14.1 \mathrm{~min} /$ day exercising and $8.21 \pm 0.7$ hours/day sleeping. Linear models revealed that exercise did not predict sleep $(\mathrm{b} 0=502, \mathrm{pEX}=.92$, pOncologist $=.51, \mathrm{pEX} *$ Oncologist $=.57)$ and sleep did not predict exercise $(\mathrm{b} 0=78, \mathrm{pSL}=.09, \mathrm{pSL}=.47, \mathrm{pEX} *$ Oncologist $=.20)$. In terms of magnitude of association, for every hour increase in exercise, oncologists spent $22 \mathrm{~min}$ less in bed the following night. For every hour increase in exercise, cardiologists spent $3.3 \mathrm{~min}$ more in bed the following night. Discussion: There were no significant associations between daily exercise and sleep, suggesting physician's sleep and exercise behaviors may be dictated by demanding work schedules. Future studies should examine how work schedules (e.g., time at work, weekday vs weekend days) influence sleep and exercise among physicians.

\section{Systematic Review of Accelerometer-Based Methods for 24-Hour Physical Behavior Assessment in Young Children (0-5-Years-Old)}

Annelinde Lettink ${ }^{1}$, Teatske Altenburg ${ }^{1}$, Jelle Arts ${ }^{1}$, Vincent van Hees ${ }^{1}$, Mai J. Chinapaw ${ }^{1}$

${ }^{1}$ Amsterdam UMC

Introduction: To our knowledge no systematic reviews exist on the measurement properties of accelerometer-based methods for monitoring 24-hour physical behaviors in 0-5-year-olds. Methods: We searched PubMed for studies examining test-retest or inter-device reliability, criterion-, convergent-, cut-point- or prediction validity of accelerometer-based methods for assessing physical activity (PA), sedentary behavior (SB) and/ or sleep. We developed a new checklist inspired by COSMIN to rate quality of evidence. Results: The 52 eligible studies examined two different analysis approaches: conventional cut-point-based methods, and more advanced methods. In infants, no cut-points exist for SB and PA, while for sleep existing cut-points were not valid. In toddlers, cut-points appeared valid for distinguishing SB and low intensity PA from high intensity PA, while no cut-points exist for sleep. In preschoolers, valid cutpoints both for hip and wrist placement for assessing SB, LPA, MVPA, and sleep were identified. In infants, one machine learning (ML) study identified an advanced method for posture, SB and PA classification, while several advanced methods could identify sleep from three months of age. In toddlers, one ML method could distinguish between SB and ambulation PA, while no studies were found on sleep. In preschoolers, several ML methods distinguished SB, LPA and MVPA in a laboratory setting but not free-living, while various advanced methods for sleep were reported. Many of the advanced methods were not open-source or commercial software. Discussion: Up to age three valid cut-points to assess 24hour physical behaviors were lacking, while only one advanced method could distinguish some waking behaviors. However, more evidence is available for preschoolers: both valid cut-points and advanced methods were identified. In young children we need more high-quality studies exploring data from multiple sensor placements and multiple axes for classifying physical behaviors. 


\section{Wearable-Specific Indicators of PA Behaviour (WIPAB): A Scoping Review}

Anne Backes ${ }^{1}$, Vincent van Hees ${ }^{2}$, Guy Fagherazzi ${ }^{1}$, Laurent Malisoux ${ }^{1}$

${ }^{1}$ Luxembourg Institute of Health, ${ }^{2}$ Amsterdam UMC

Introduction: Physical activity (PA) is a complex human behaviour, which raises the question whether solely the Frequency, Intensity, Time and Type of PA (FITT framework) are sufficient to describe it. The FITT framework has been great for questionnaire research, but with rise of wearable sensors as default research tool, additional dimensions of PA behaviour may be identified and captured. This scoping review aimed to identify those analytical methods and their summary variables that can only be captured with a wearable, which were here defined as wearablespecific indicators of PA behaviour (WIPAB). Methods: Three databases (MEDLINE, Embase and Web of Science) were screened for articles published in English between January 2010 and April 2020. Articles, which objectively assessed PA behaviour by using tri-axial acceler- ometers and which investigated WIPAB were selected. An overview of the identified WIPAB was generated, as well as a synthesis of the approaches. Results: From the 24 articles included in the review, 13 WIPAB were identified. They can be classified into three different categories focussing on 1) activity intensity distribution, 2) activity accumulation and 3) temporal correlation and regularity of the acceleration signal. The first category provides information on the distribution of the activity intensities across 24 hours. The second category demonstrates how an individual accumulates activity or sedentary time by analysing the distribution or proportion of shorter and longer bout lengths. The third category quantifies how regular or complex a PA behaviour is, through the analysis of correlations between values or the presence and reoccurrence of sub-patterns in the time series. Only 5 of these WIPAB have been used in health research so far. Discussion: The WIPAB may provide important information about complex PA behaviour, however more research is needed to understand their added value and practical feasibility relative to the FITT framework. (Figure 72) 


\section{Appendix}
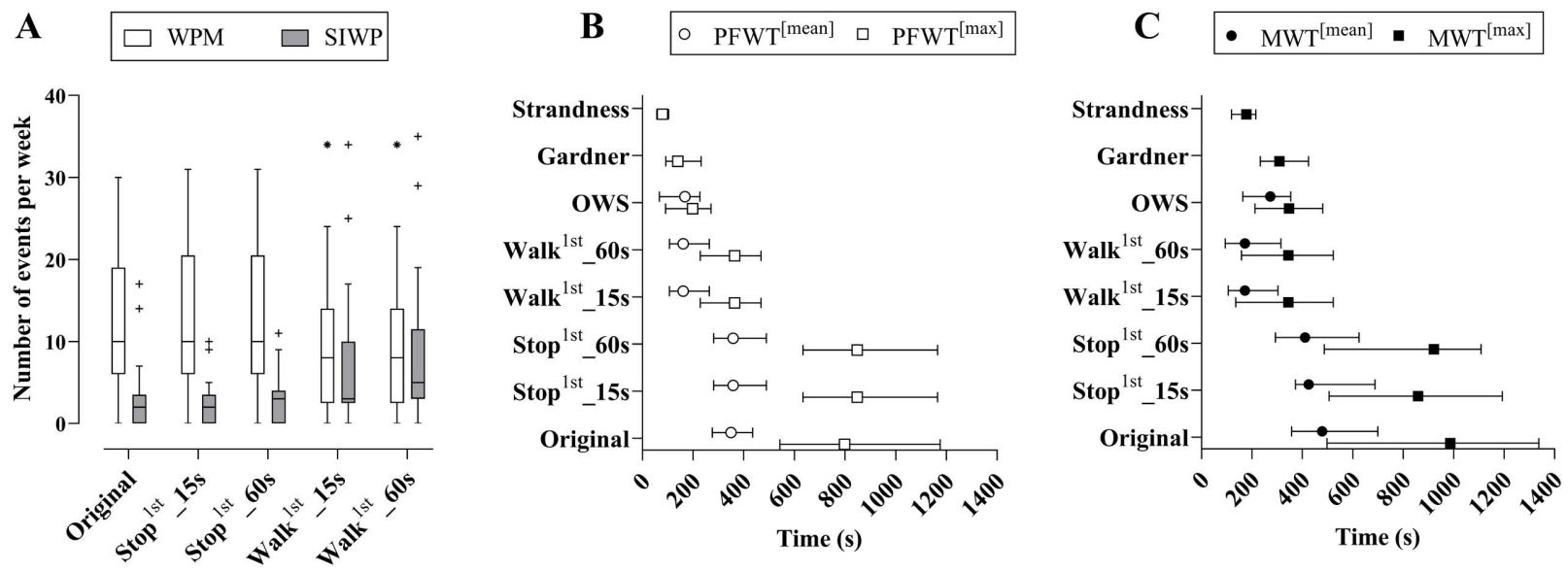

Figure 1. Panel A: Boxplots of the number per week of walking pain manifestation and stop-induced by walking pain when considering our previously published algorithm (Original), removing short stopping bouts first with a delay of $15 \mathrm{~s}$ or $60 \mathrm{~s}$ (Stop ${ }^{1 \mathrm{st}}{ }_{-} 15 \mathrm{~s} / 60 \mathrm{~s}$ ), and removing short walking bouts first with a delay of $15 \mathrm{~s}$ or $60 \mathrm{~s}$ (Walk ${ }^{1 \mathrm{st}} 15 \mathrm{~s} / 60 \mathrm{~s}$ ); Panels B and $\mathrm{C}$ : Medians and interquartile ranges of the pain-free walking times and maximal walking times presented by their maximal and mean values in each context of measurement and for each algorithm applied for the free-living measurement in PAD participants.

MWT, maximal walking time; OWS, outdoor walking session; PFWT, pain-free walking time; SIWP, stop-induced by walking pain; WPM, walking pain manifestation.

Figure 1 

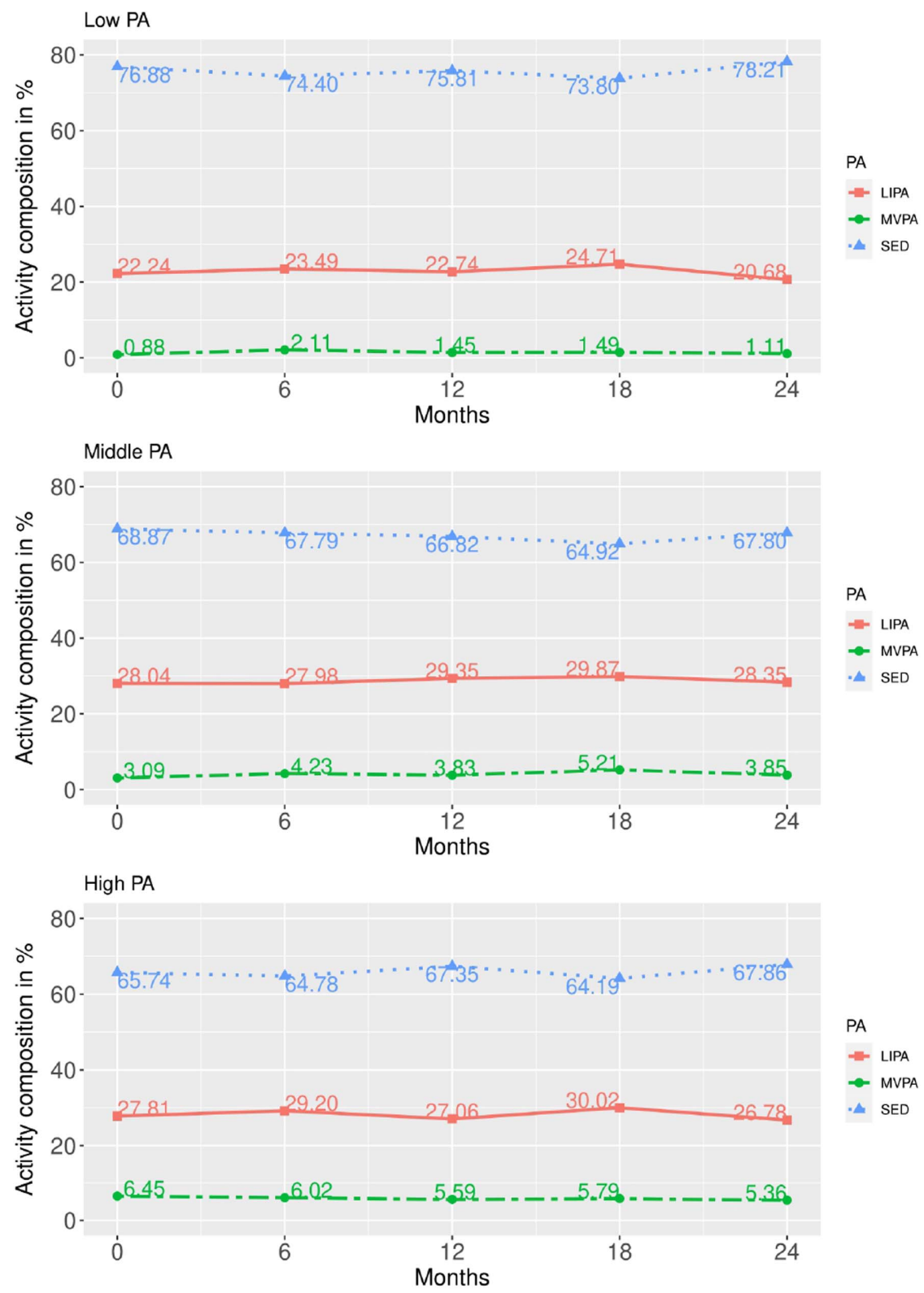

Figure 1. The activity composition in percentage for the low, middle, and high PA groups at $0,6,12,18$ and 24 months.

Figure 2 
Table 1. Top ten ranked models in discriminating fallers and non-fallers in Parkinson's disease. $A B C$ Scale, Activities-Specific Balance Confidence Scale; AUC, area under the curve; BIC, Bayesian information criterion; MSD-UPDRS PIGD, Movement Disorders Society Unified Parkinson's Disease Rating Scale Postural Instability and Gait Disorders; NFoGQ, New Freezing of Gait Questionnaire; RMS-AP EOfoam, root mean square of anterior-posterior trunk acceleration while standing on a foam with eyes open; ROM, range of motion; SCOPA-COG, Scales for Outcomes in Parkinson's Disease-Cognition.

\begin{tabular}{|c|c|c|c|c|c|c|c|}
\hline \multicolumn{4}{|c|}{ Outcomes } & \multirow{2}{*}{ AUC } & \multirow{2}{*}{ BIC } & \multirow{2}{*}{\multicolumn{2}{|c|}{ Specificity Sensitivity }} \\
\hline 1st & 2nd & 3rd & 4th & & & & \\
\hline $\begin{array}{l}\text { NFoGQ } \\
\text { past month }\end{array}$ & $\begin{array}{l}\text { Gait double } \\
\text { support }\end{array}$ & $\begin{array}{l}\text { Turn duration } \\
\text { variability }\end{array}$ & $\begin{array}{l}\text { RMS-AP } \\
\text { EOfoam }\end{array}$ & $\begin{array}{c}0.94 \\
(0.83-1)\end{array}$ & -22.22 & 1.00 & 0.78 \\
\hline $\begin{array}{l}\text { NFoGQ } \\
\text { past month }\end{array}$ & SCOPA-COG & $\begin{array}{l}\text { Gait double } \\
\text { support }\end{array}$ & $\begin{array}{l}\text { RMS-AP } \\
\text { EOfoam }\end{array}$ & $\begin{array}{c}0.90 \\
(0.74-1)\end{array}$ & -22.85 & 0.86 & 1.00 \\
\hline $\begin{array}{l}\text { NFoGQ } \\
\text { past month }\end{array}$ & SCOPA-COG & & & $\begin{array}{c}0.87 \\
(0.70-0.99)\end{array}$ & -17.09 & 0.73 & 1.00 \\
\hline $\begin{array}{l}\text { MDS-UPDRS } \\
\text { PIGD score }\end{array}$ & $\begin{array}{l}\text { NFoGQ } \\
\text { past month }\end{array}$ & Step duration & & $\begin{array}{c}0.84 \\
(0.67-0.97)\end{array}$ & -19.29 & 0.88 & 0.70 \\
\hline $\begin{array}{l}\text { Turn duration } \\
\text { variability }\end{array}$ & & & & $\begin{array}{c}0.84 \\
(0.66-0.96)\end{array}$ & -2.38 & 0.89 & 0.70 \\
\hline $\begin{array}{l}\text { NFoGQ } \\
\text { past month }\end{array}$ & $\begin{array}{l}\text { Gait double } \\
\text { support }\end{array}$ & $\begin{array}{l}\text { Trunk sagittal } \\
\text { ROM }\end{array}$ & $\begin{array}{l}\text { RMS-AP } \\
\text { EOfoam }\end{array}$ & $\begin{array}{c}0.83 \\
(0.64-0.98)\end{array}$ & -22.55 & 0.75 & 0.89 \\
\hline $\begin{array}{l}\text { MDS-UPDRS } \\
\text { PIGD score }\end{array}$ & & & & $\begin{array}{c}0.83 \\
(0.66-0.97)\end{array}$ & -9.50 & 0.78 & 0.80 \\
\hline $\begin{array}{l}\text { MDS-UPDRS } \\
\text { PIGD score }\end{array}$ & $\begin{array}{l}\text { NFoGQ } \\
\text { past month }\end{array}$ & & & $\begin{array}{c}0.83 \\
(0.66-0.96)\end{array}$ & -19.20 & 0.94 & 0.60 \\
\hline $\begin{array}{l}\text { MDS-UPDRS } \\
\text { PIGD score }\end{array}$ & $\begin{array}{l}\text { NFoGQ } \\
\text { past month }\end{array}$ & $\begin{array}{l}\text { RMS-AP } \\
\text { EOfoam }\end{array}$ & & $\begin{array}{c}0.82 \\
(0.63-0.96)\end{array}$ & -19.84 & 0.50 & 1.00 \\
\hline ABC scale & $\begin{array}{l}\text { NFoGQ } \\
\text { past month }\end{array}$ & $\begin{array}{l}\text { Gait double } \\
\text { support }\end{array}$ & $\begin{array}{l}\text { RMS-AP } \\
\text { EOfoam }\end{array}$ & $\begin{array}{c}0.81 \\
(0.56-1)\end{array}$ & -21.69 & 0.88 & 0.88 \\
\hline
\end{tabular}

Figure 3

Blue: clinical measure; grey: gait measure; yellow: balance measure.

Table 1: Sleep-related parameters using an unobtrusive bed sensor for iSCl and stroke patients, indicated as mean (SD) or as count.

\begin{tabular}{|l|l|l|l|}
\hline & iSCI (n=11) & Stroke (n=9) & $P$ value \\
\hline Hours of sleep (h, min) & $8 \mathrm{~h}, 3 \mathrm{~min}(1 \mathrm{~h}, 8 \mathrm{~min})$ & $8 \mathrm{~h}, 10 \mathrm{~min}(30 \mathrm{~min})$ & .74 \\
\hline $\begin{array}{l}\text { Self-rep orted sleep score } \\
\text { (NRS) }\end{array}$ & $6(1)$ & $7.6(1.1)$ & .003 \\
\hline Noctumal heart rate (bpm) & $72(13)$ & $61(8)$ & .04 \\
\hline $\begin{array}{l}\text { Noctumal respiratory rate } \\
\text { (cpm) }\end{array}$ & $16(3)$ & $15(1)$ & .21 \\
\hline $\begin{array}{l}\text { Noctumal movement } \\
\text { activity (activity points) }\end{array}$ & $239(116)$ & $136(49)$ & .02 \\
\hline
\end{tabular}

iSCI = incomplete sp inal cord injury; $\mathrm{h}=$ hours; $\mathrm{min}=$ minutes; $\mathrm{NRS}=$ numeric rating scale; $\mathrm{bpm}=$ beats per minute; $\mathrm{cpm}=\mathrm{cycles}$ per

Figure 4 minute. 

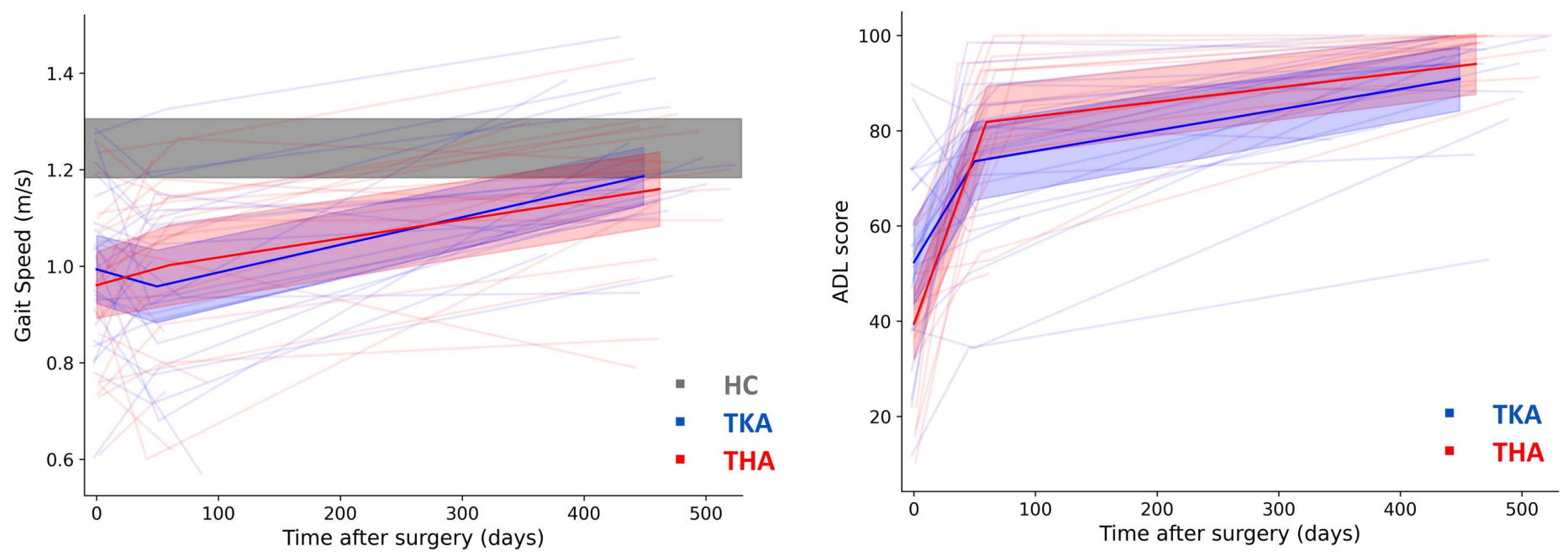

Figure 1: Recovery patterns of gait speed (left) and self-reported outcomes, i.e. the HOOS/KOOS subscale for activities of daily living (ADL) (right), with 0 representing extreme difficulty with an activity and 100 representing no difficulty. Individual trajectories are presented together with group mean (bold line) and the $95 \%$ confidence interval (shaded areas).

Figure 5

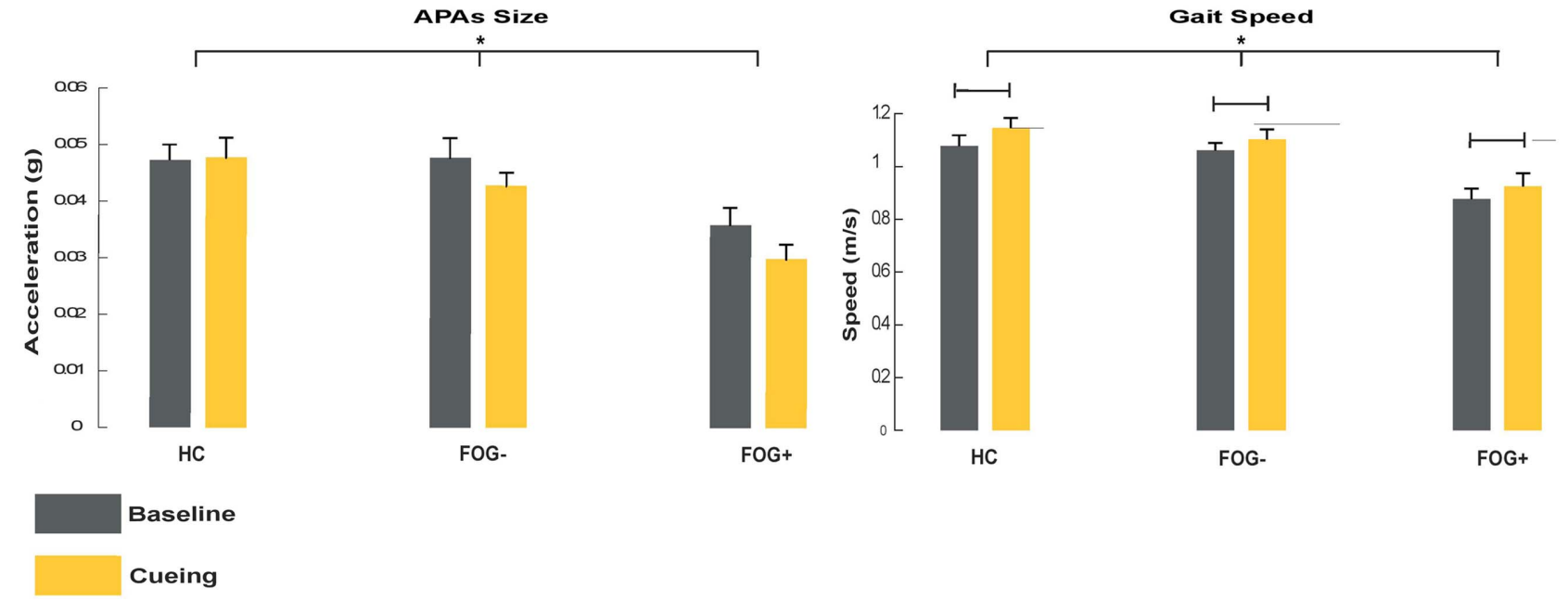

Figure 6 


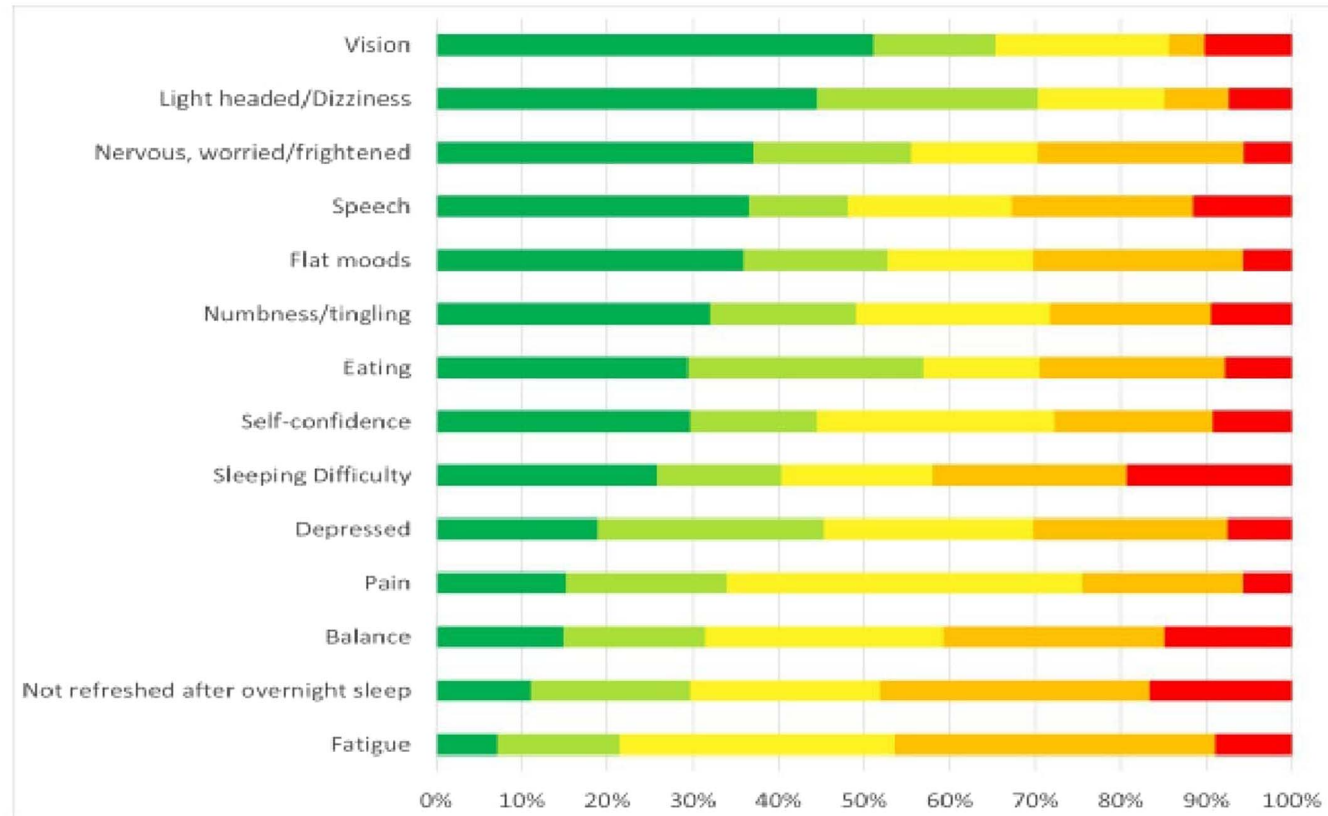

Figure 1. Non-motor symptoms during COVID assessed by the Non-motor Symptom Scale rated by a 1-5 scale. 1 (green) = no symptom, 5 (red) $=$ worse possible symptom

\section{References:}

1. Chen PM, Hemmen TM. Evolving Healthcare Delivery in Neurology During the Coronavirus Disease 2019 (COVID-19) Pandemic. Front Neurol. 2020;11:578. Published 2020 May 29. doi:10.3389/fneur.2020.00578

2. Zullo, S., Ingravallo, F., Crespi, V., Cascioli, M., D'Alessandro, R., Gasperini, M., Lalli, C., Lugaresi, A., Marogna, M., Mori, M., Pesci, I., Pistoia, F., Porteri, C., Vedovello, M., Veronese, S., Pucci, E., Solari, A., \& Gruppo di Studio "Bioetica e Cure Palliative" of the Italian Neurological Society (2021). The impact of the COVID-19 pandemic on people with neurological disorders: an urgent need to enhance the health care system's preparedness. Neurological sciences: official journal of the Italian Neurological Society and of the Italian Society of Clinical Neurophysiology, 42(3), 799-804. https://doi.org/10.1007/s10072-020-04984-4

Figure 7

Table 1. Median (interquartile range) number and length of bouts and duration per day of total activity and MVPA

\begin{tabular}{|c|c|c|c|c|c|c|}
\hline & \multicolumn{3}{|c|}{ Total activity } & \multicolumn{3}{|c|}{ MVPA only } \\
\hline & $\mathrm{n}$ bouts/day & Bout length (min) & $\begin{array}{l}\text { Duration } \\
\text { (min/day) }\end{array}$ & $\mathrm{n}$ bouts/day & Bout length (min) & $\begin{array}{l}\text { Duration } \\
\text { (min/day) }\end{array}$ \\
\hline$\geq 2 \min$ & $39.0(12.3)$ & $4.0(1.0)$ & $249.3(115.9)$ & $13.0(8.1)$ & $3.0(0.5)$ & $65.3(59.8)$ \\
\hline$\geq 3 \mathrm{~min}$ & $27.0(9.0)$ & $5.0(1.5)$ & $219.8(119.9)$ & $8.0(7.0)$ & $4.1(1.0)$ & $55.5(57.3)$ \\
\hline$\geq 4 \min$ & $20.0(8.0)$ & $6.5(1.0)$ & $197.5(115.3)$ & $6.0(6.0)$ & $6.0(1.5)$ & $45.8(51.1)$ \\
\hline$\geq 5 \min$ & $15.0(7.3)$ & $8.0(2.0)$ & $178.3(110.9)$ & $4.0(4.0)$ & $7.0(1.5)$ & $40.0(46.6)$ \\
\hline$\geq 6 \mathrm{~min}$ & $11.8(6.3)$ & $9.1(1.5)$ & $160.3(108.0)$ & $3.0(3.0)$ & $8.3(1.0)$ & $35.3(41.5)$ \\
\hline$\geq 7 \mathrm{~min}$ & $10.0(5.1)$ & $11.0(1.6)$ & $144.3(105.8)$ & $2.0(2.0)$ & $10.0(2.0)$ & $34.3(35.9)$ \\
\hline$\geq 8 \mathrm{~min}$ & $8.0(5.1)$ & $12.0(2.0)$ & $130.0(100.6)$ & $1.8(2.0)$ & $11.0(2.0)$ & $33.3(34.0)$ \\
\hline$\geq 9 \min$ & $7.0(5.0)$ & $13.0(1.6)$ & $120.5(99.4)$ & $1.0(2.0)$ & $12.5(1.8)$ & $31.8(30.3)$ \\
\hline$\geq 10 \mathrm{~min}$ & $5.5(4.5)$ & $14.5(1.6)$ & $107.5(95.1)$ & $1.0(2.0)$ & $13.6(2.5)$ & $31.0(30.9)$ \\
\hline Total (unbouted) & -- & -- & $290.0(115.6)$ & -- & -- & $78.0(68.5)$ \\
\hline
\end{tabular}

Note. Total activity $=$ light, moderate, vigorous physical activity. MVPA $=$ moderate, vigorous physical activity.

Figure 8 
Table 1. Crude and adjusted association between maternal and paternal MVPA and 4-yearold child overall PA. Pelotas, 2015 birth cohort.

\begin{tabular}{|c|c|c|c|c|c|c|c|}
\hline & & \multicolumn{6}{|c|}{ Child Overall PA (Acceleration ENMO - mg) } \\
\hline & & \multicolumn{3}{|c|}{ Crude } & \multicolumn{3}{|c|}{ Adjusted } \\
\hline & & Mean & $95 \% \mathrm{Cl}$ & P-value* & Mean & $95 \% \mathrm{Cl}$ & P-value* \\
\hline Maternal MVPA (tertiles) & & & & 0.004 & & & $0.021^{\varepsilon}$ \\
\hline $\mathrm{T} 1$ & 695 & 47.3 & $46.5 ; 48.2$ & & 47.6 & $46.7 ; 48.5$ & \\
\hline $\mathrm{T} 2$ & 698 & 48.7 & $47.8 ; 49.5$ & & 48.7 & $47.8 ; 49.5$ & \\
\hline T3 & 694 & 49.4 & $48.5 ; 50.2$ & & 49.1 & $48.2 ; 49.9$ & \\
\hline Paternal MVPA (tertiles) & & & & 0.01 & & & $0.012^{\#}$ \\
\hline $\mathrm{T} 1$ & 583 & 47.1 & $46.1 ; 48$ & & 47.2 & $46.2 ; 48.1$ & \\
\hline $\mathrm{T} 2$ & 550 & 47.4 & $46.5 ; 48.4$ & & 47.5 & $46.5 ; 48.5$ & \\
\hline T3 & 582 & 48.9 & $48 ; 49.9$ & & 49.0 & $48 ; 49.9$ & \\
\hline Mother and Father in T3 & & & & $<0.001$ & & & $0.001^{\epsilon}$ \\
\hline No & 634 & 47.0 & $46.1 ; 47.8$ & & 47.0 & $46.2 ; 47.9$ & \\
\hline Yes & 178 & 50.1 & $48.6 ; 51.7$ & & 50.4 & $48.6 ; 52.1$ & \\
\hline
\end{tabular}

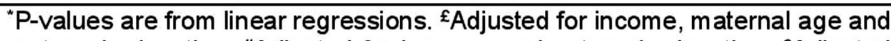
maternal education. "Adjusted for income, and paternal education. ${ }^{\epsilon}$ Adjusted for income, maternal age, paternal education, and matemal education

Figure 9

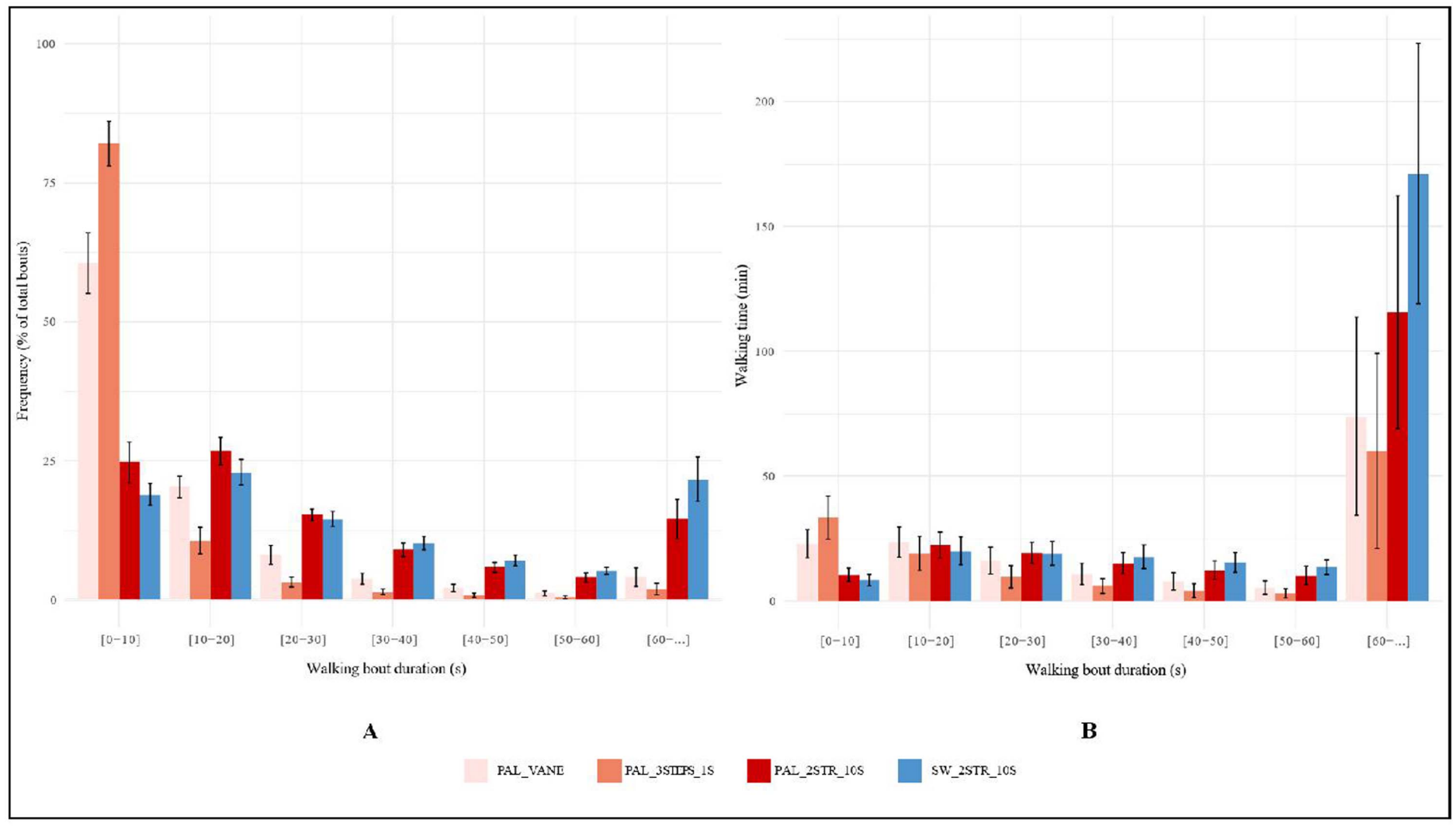

Figure 1. Frequency (A) and Walking Time (B) distributions of walking bouts duration for all subjects, according to the methods and monit ors used. Figure 10 
Figure 1. Self-Reported Sedentary Activities Stratified by activPAL-based Sitting Pattern Metrics.

A

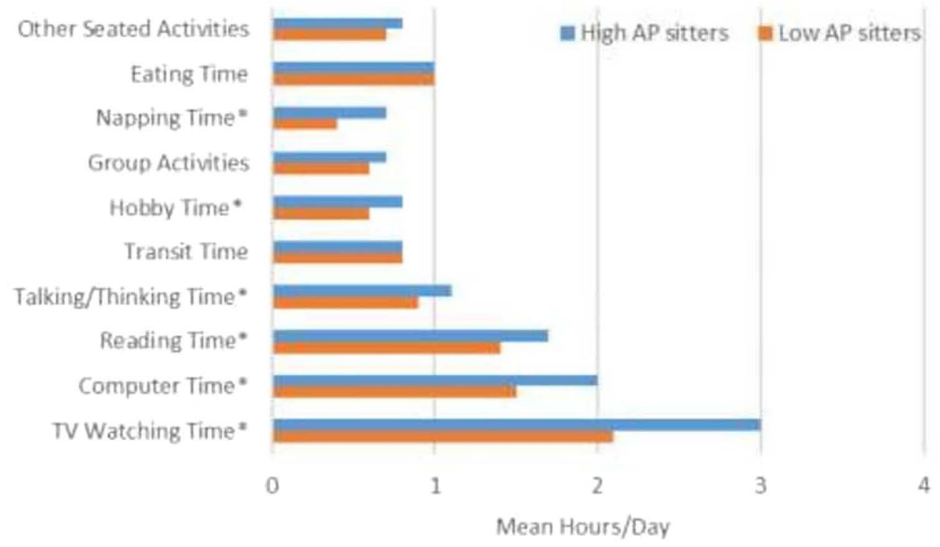

B

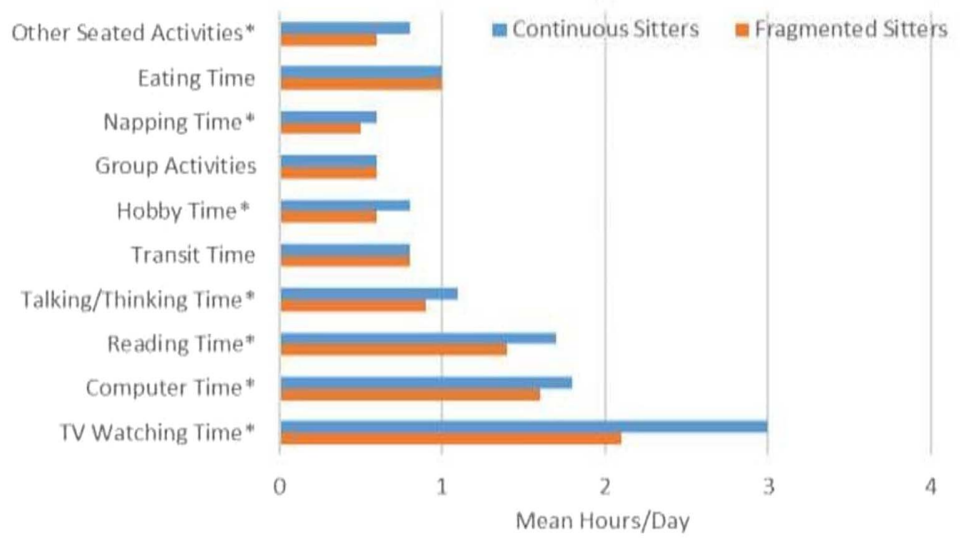

${ }^{*} p<0.05$

AP = activPAL. A) High vs. Low AP sitters are defined by the sample's median activPAL-measured total daily sitting time (10.0 hours/day). B) Fragmented vs. Continuous sitters are defined by the sample's median activPAL-measured mean sitting bout duration (14.0 mins/bout).

Figure 11

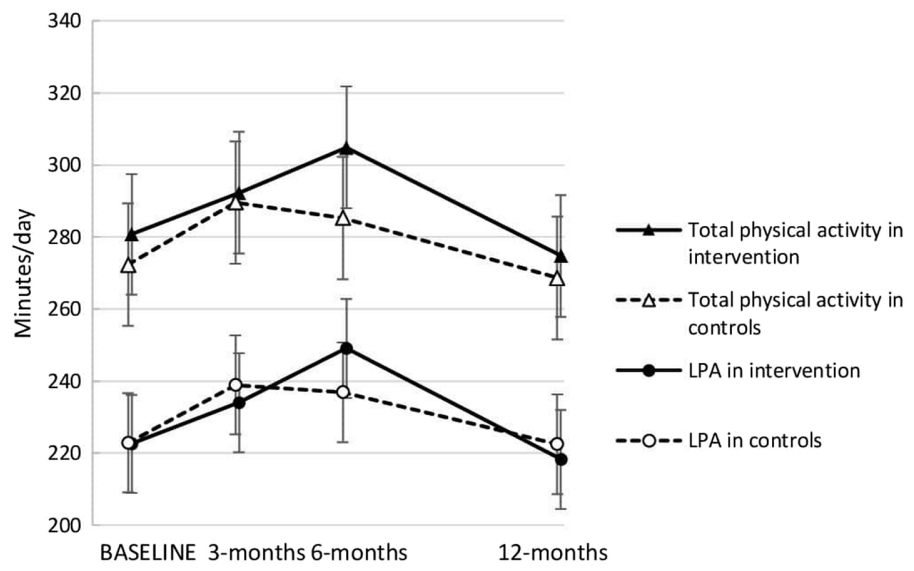

Figure 12 
Figure 1. Standardised effect estimates of the association between physical activity levels and brain structures.

A

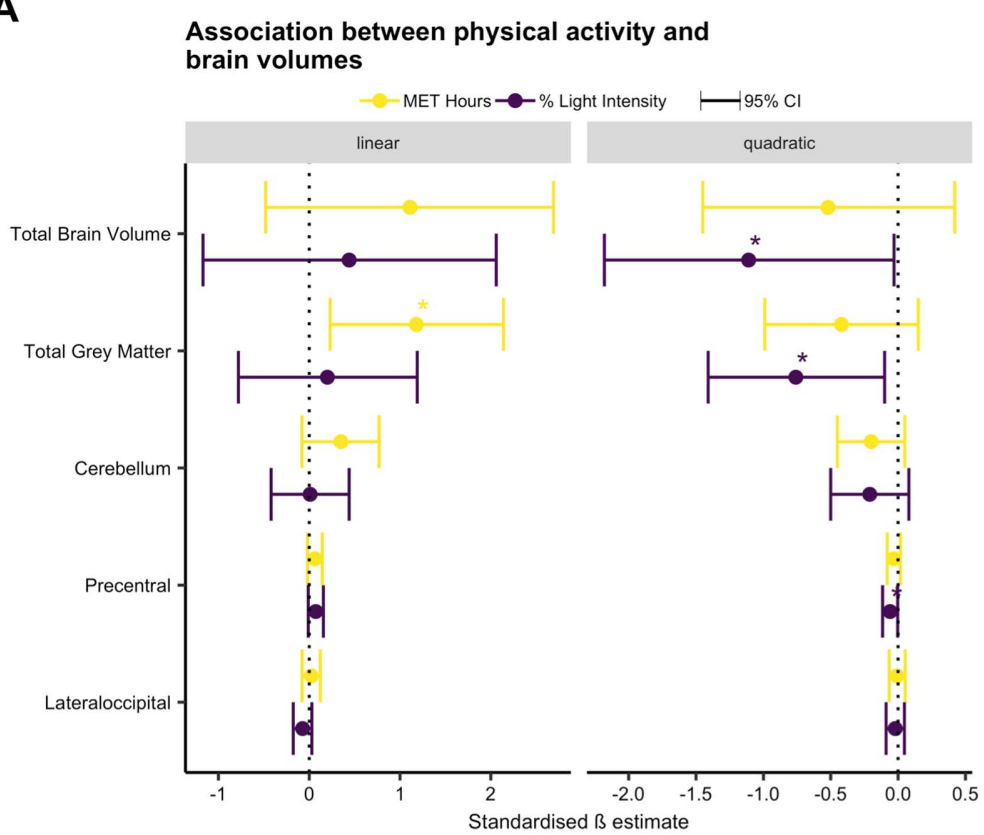

C

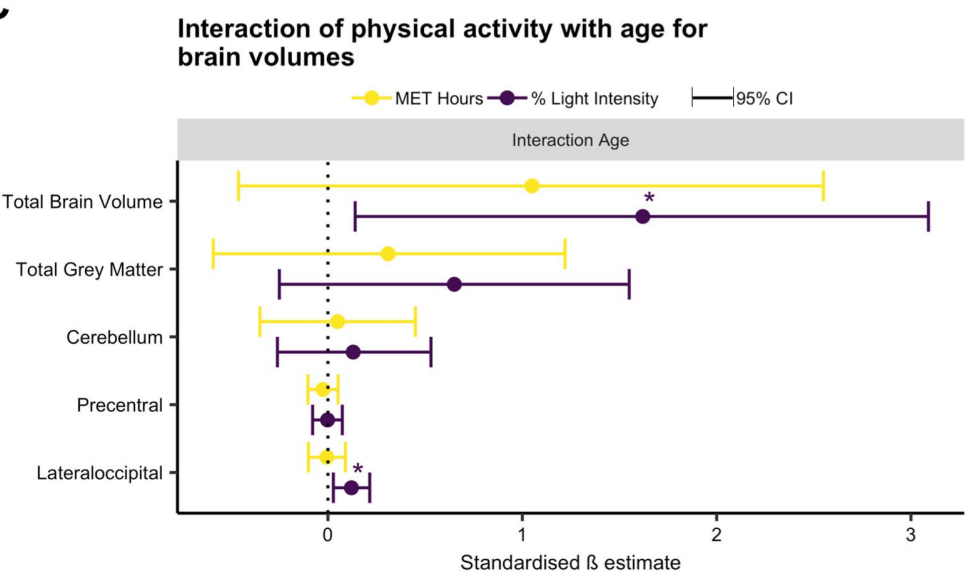

B

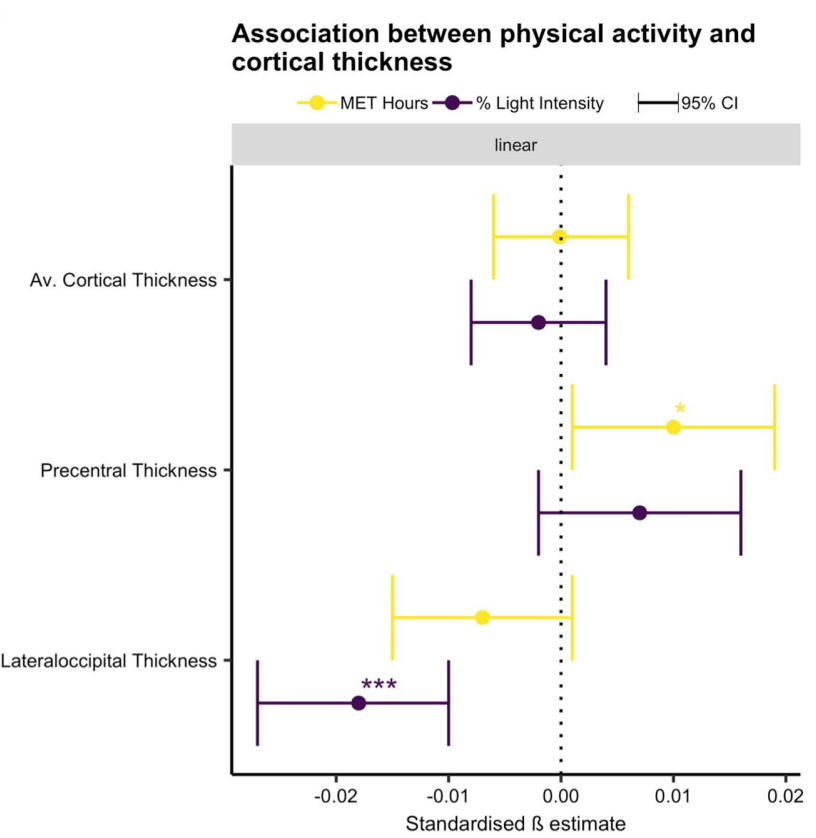

D

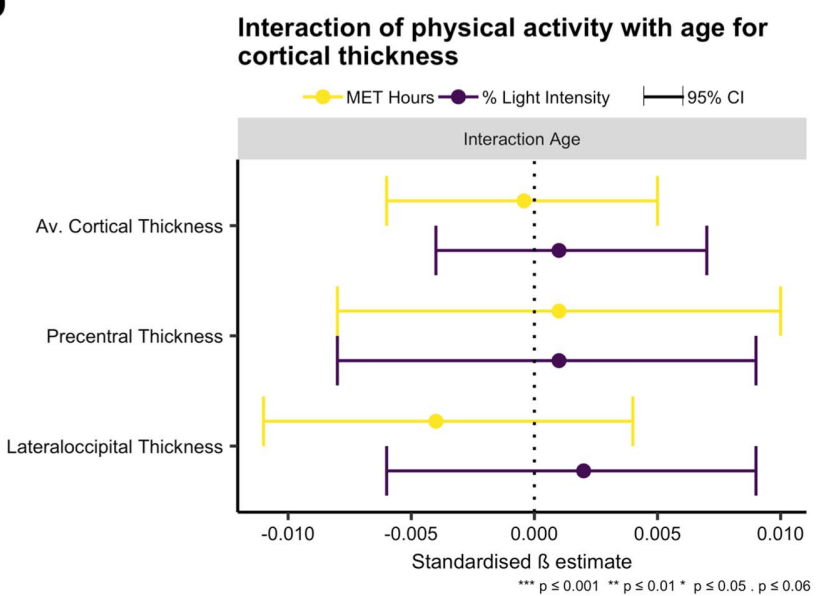

Figure 13 
Table 1: Area Under the Curve (AUC) with $95 \%$ confidence interval $(95 \% \mathrm{Cl}$ ) from receiver

operating characteristic curves discriminating between people with mild traumatic brain injury

and healthy controls on metrics of continuous passive monitoring - turning and activity. Effect

sizes and $p$ values are presented from independent sample t-tests unless noted otherwise.

Sizes and $p$ values are presented from independent sample t-tests unless noted otherwise.

Turning Metrics

Number of Turns/Hour

Average Turn Angle Magnitude

Turn Angle Magnitude Variability

Average Turn Duration

Turn Duration Variability

Average Peak Turn Velocity

Peak Turn Velocity Variability

Average Turn Velocity

Average Turn Velocity Variability

Activity Metrics

Average Number of Daily Bouts

Average Bout Duration

Bout Duration Variability

Average Number of Steps/Bout

Average Daily Activity Rate

$95 \% \mathrm{Cl}$ ERI

$p$ value

0.66

$0.27^{* a}$

0.63

$(0.47,0.79)$

0.056

0.62

$0.35^{\star}$

0.215

$(0.45,0.78)$

$0.42^{*}$

0.138

0.61

$(0.43,0.78)$

0.63
$0.47,0.79)$

0.18

0.207

0.54

$-0.64^{\star *}$

0.026

0.54
$(0.37,0.71)$

-0.21 *

0.464

0.66

$-0.71^{\text {** }}$

0.015

0.51

$(0.34,0.68)$

$-0.11$

0.689

0.61

$-0.46^{*}$

0.107

(0.45 0.77)

$0.29^{* a}$

0.041

$0.51,0.83$

0.62

$(0.46,0.77)$

0.59

$(0.43,0.75)$

$-0.20^{\text {*a }}$

0.162

$-0.29^{*}$

0.298

0.65

$(0.50,0.81)$

0.57

(0.41, 0.73)

$0.26^{\star a}$

0.063

$0.12^{\mathrm{a}} \quad 0.389$

Average Number of Steps/Day

0.49

NE

NE

( reported from a Mann Whitney U test; NE - not estimatable; Bolded values indicate a

significant group difference $(p<0.05)$

Figure 14

Figure 1. Child PA total, week and weekend for all years

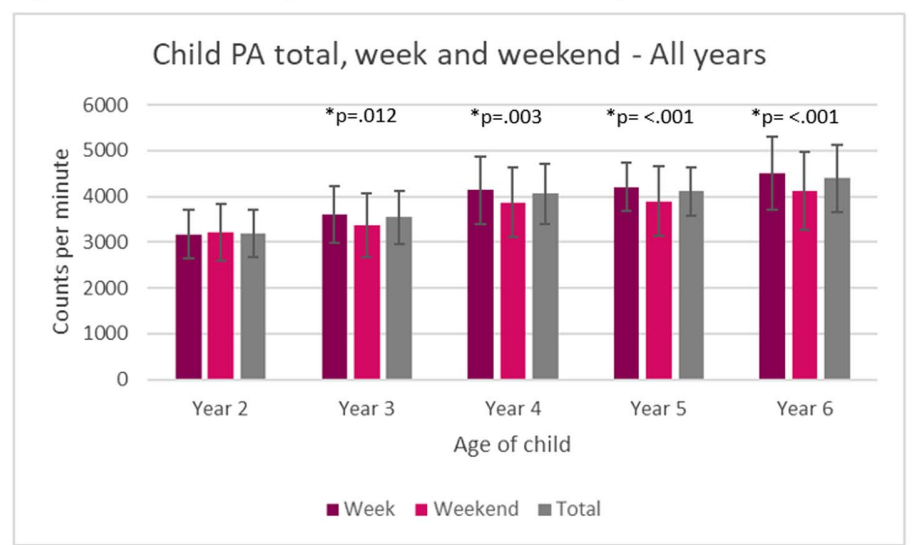

*P value significant for differences between weekdays and weekend days

Error bars represents standard deviations.

Figure 15 


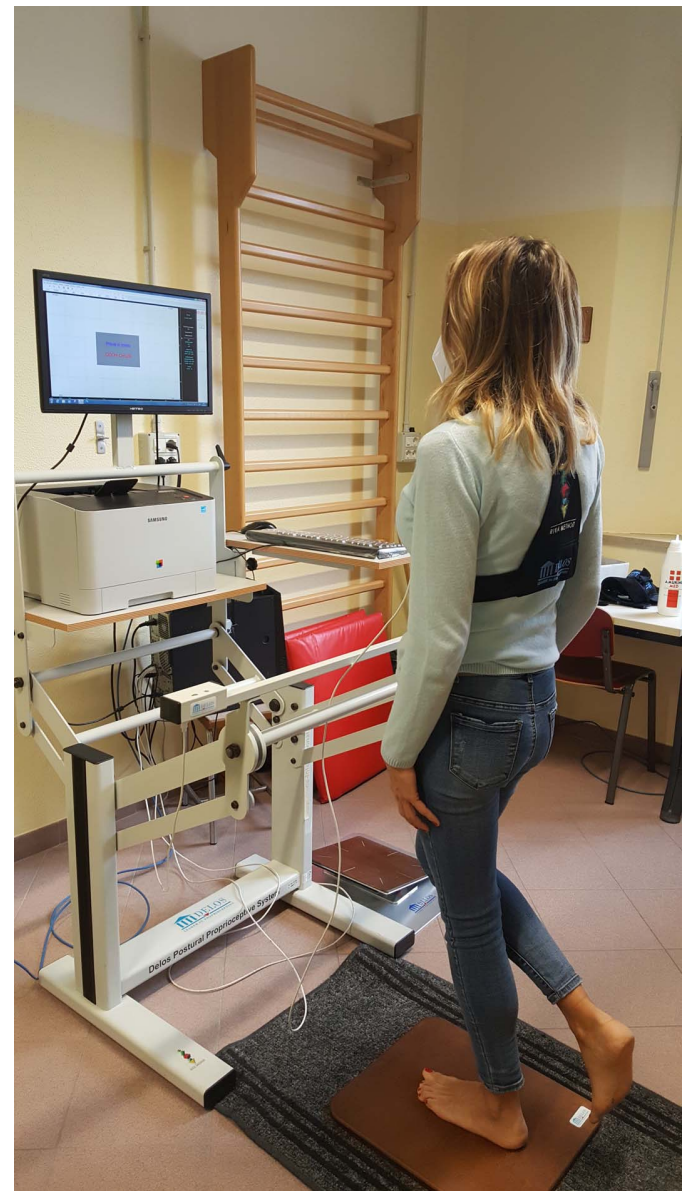

Figure 16
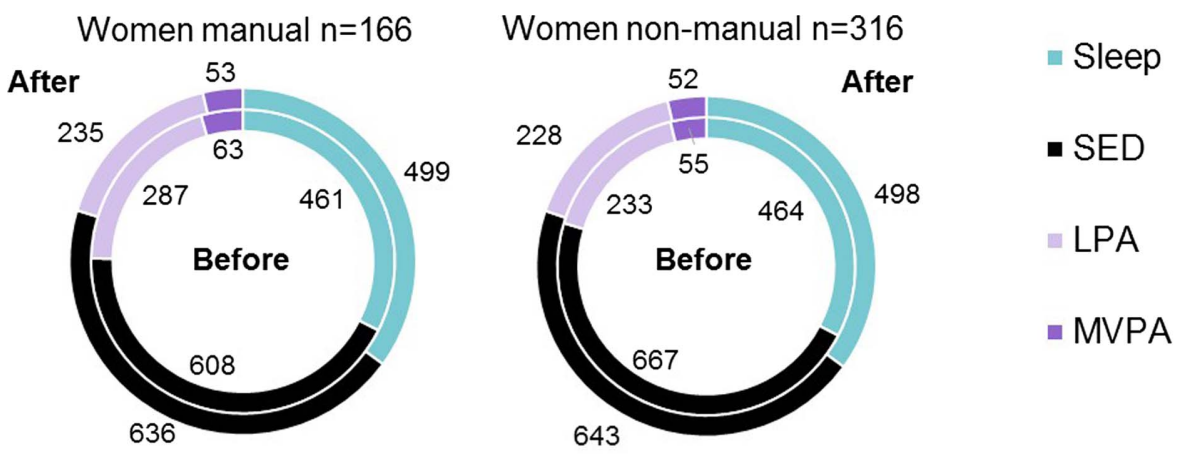

Men manual $n=27$

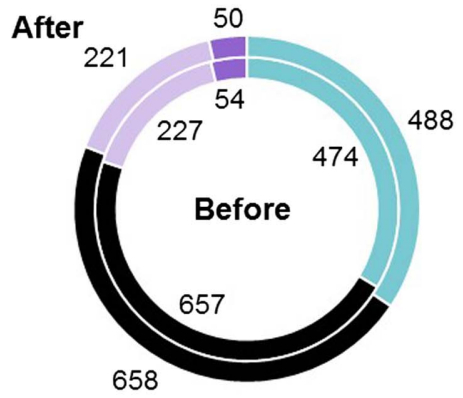

Men non-manual $n=60$

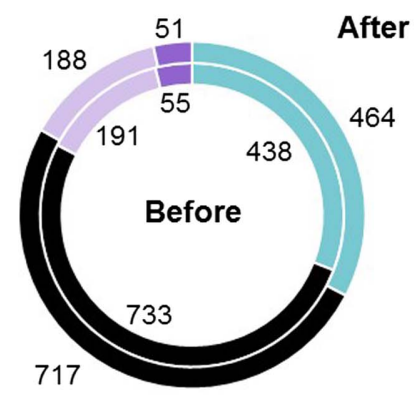

Figure 1. Sleep time, sedentary time, time spent in light physical activity (LPA) and moderate-to-vigorous physical activity (MVPA) in minutes per 24-hour day before and after the transition to retirement by gender and occupation.

Figure 17 
Table: Associations between circadian rest-activity rhythm parameters and falls $(\mathrm{N}=625)$.

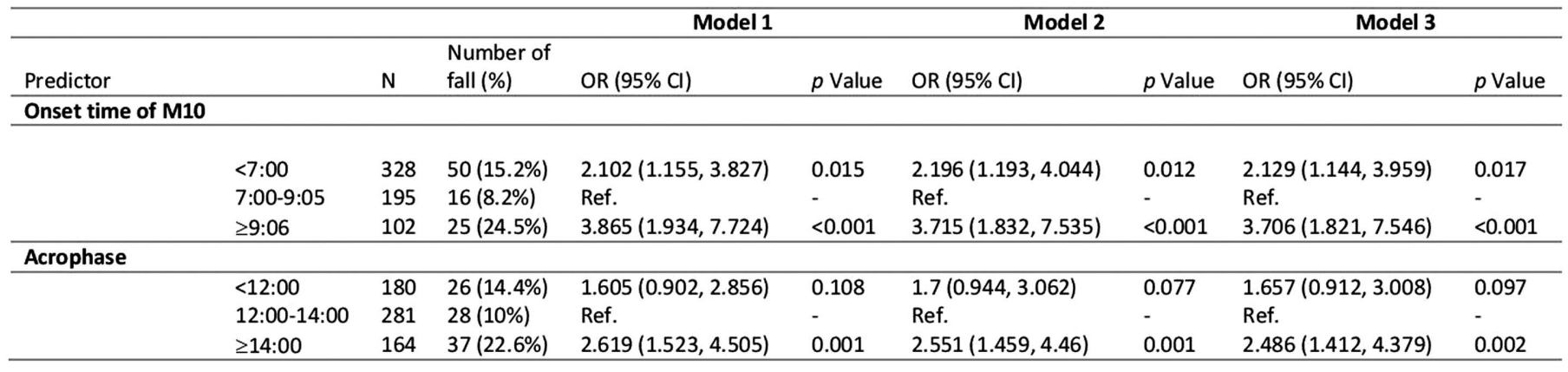

M10: most active 10 hours

Model 1: adjusted for age and gender.

Model 2: adjusted for covariates in model 1 and body mass index, number of diseases, six-meter walking speed, alcohol drinking, history of fall, cognitive impairment and depression.

Model 3: adjusted for covariates in model 2 and sleep duration and complaints.

Figure 18

Figure 19

\begin{tabular}{|c|c|c|c|c|}
\hline & \multicolumn{3}{|c|}{ Stepping Bout Duration } \\
\hline & & $10-60$ seconds & 1-15 Minutes & $>15$ Minutes \\
\hline \multirow{3}{*}{ 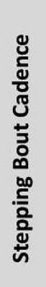 } & స్ํ ह & $22(55)$ & $136(432)$ & $\begin{array}{c}331(1,009) \\
\text { (exercise) }\end{array}$ \\
\hline & $\begin{array}{l}\text { సิ } \\
\substack{1 \\
1 \\
n}\end{array}$ & $1,947(960)$ & $\begin{array}{c}1,619(1,259) \\
\text { (outdoor) }\end{array}$ & 400 (791) \\
\hline & $\stackrel{n}{\stackrel{n}{v}} \frac{E}{n}$ & $\begin{array}{c}1,823(722) \\
\text { (indoor) }\end{array}$ & $278(240)$ & $2(22)$ \\
\hline
\end{tabular}

Table 1: Distribution of mean (standard deviation) daily step count using a cadence and duration based classification heuristic

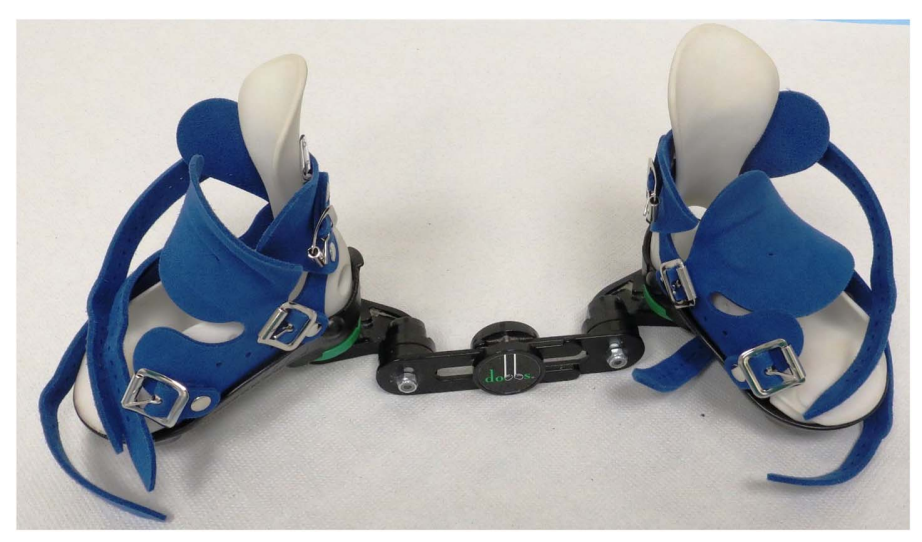

Figure 20 


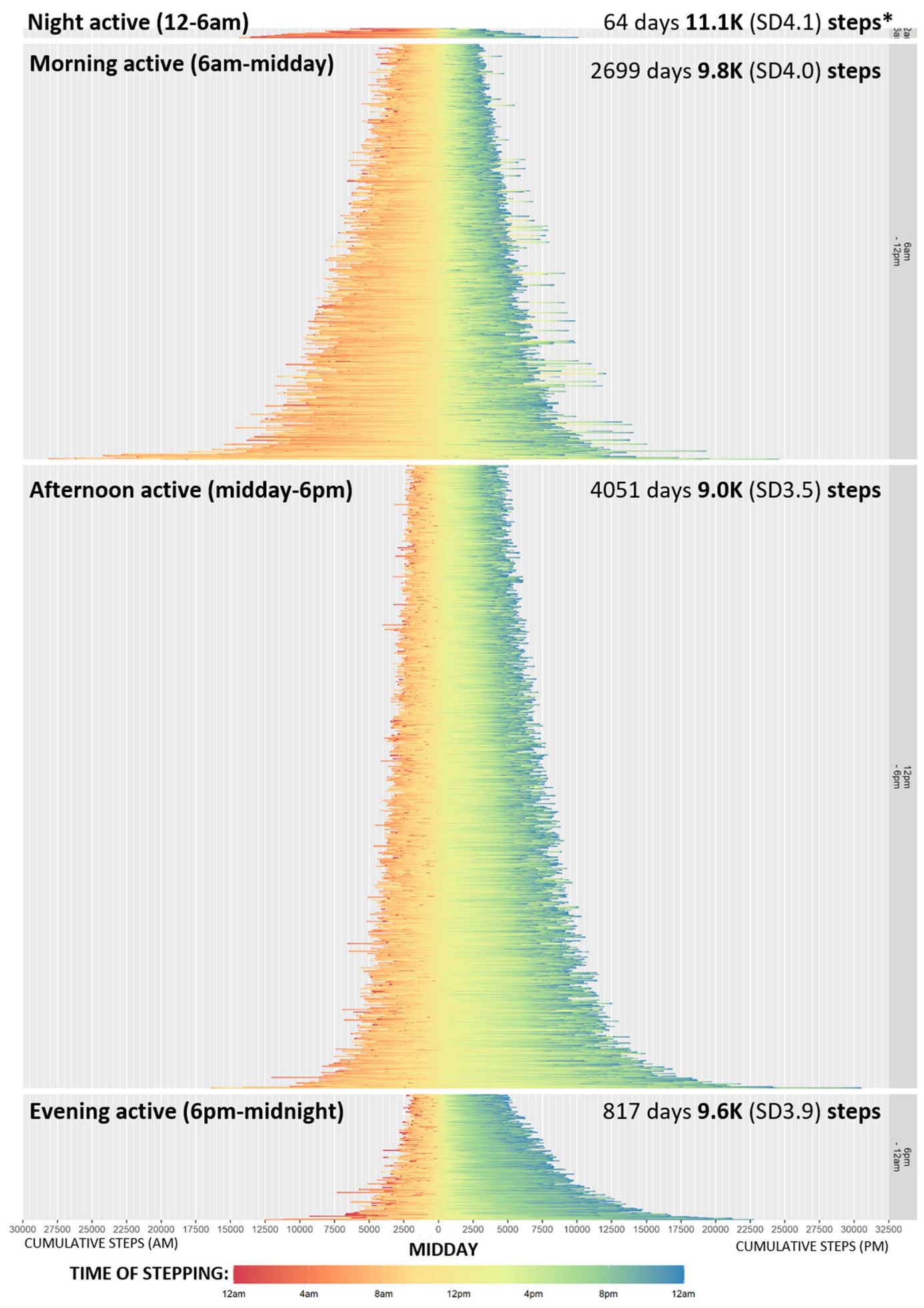

Figure 21 

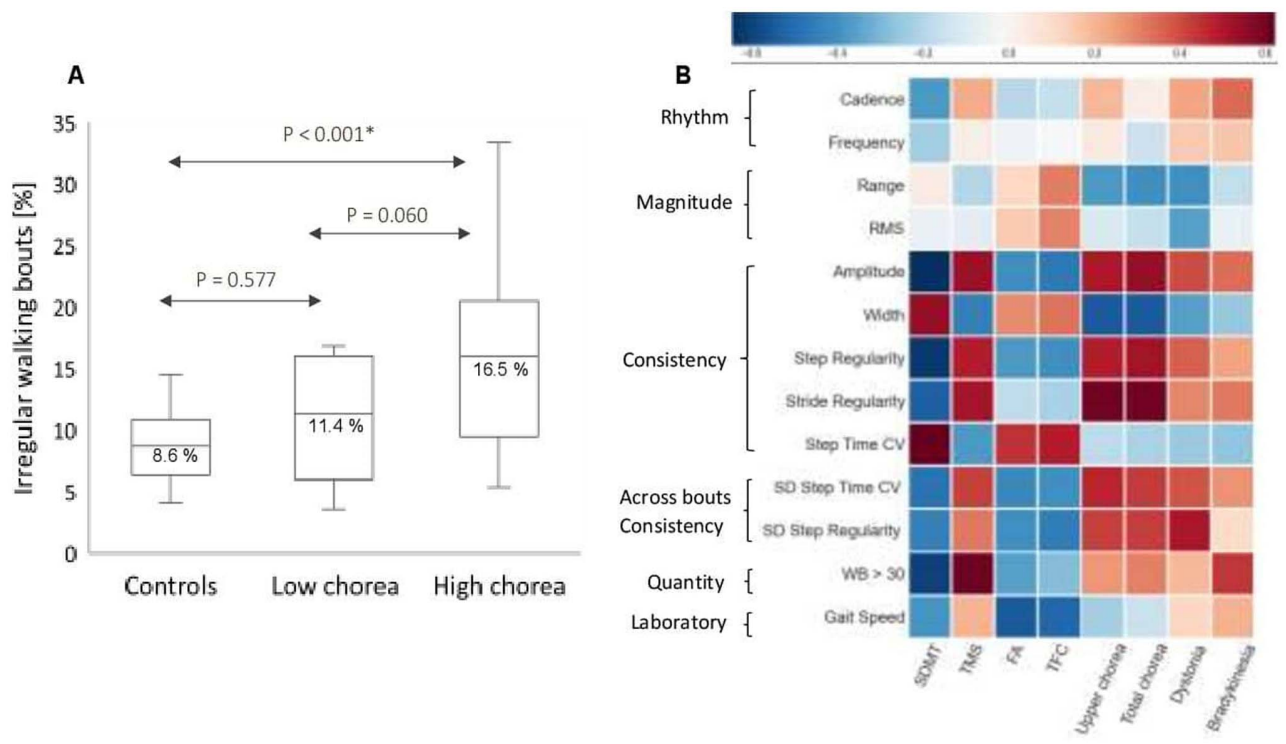

Figure 1A: Box plots of the percentage of irregular walking bouts (WB) from the total count of WB among the healthy controls and the HD participants with high upper body chorea score (High) and with low upper body chorea score (Low); box plots present the median, interquartile range (IQR), upper limit (third quartile + $1.5 * \mathrm{IQR}$ ), and lower limit (first quartile $-1.5 * \mathrm{IQR}$ ); The percentage of walking bouts identified as associated with irregular movements was greater in participants with greater levels of observer-rated upper body chorea.* Significant after Bonferroni correction for multiple comparisons. Figure 1B: Heat map showing the Spearman correlation coefficients between gait quality measures and HD clinical scores, after adjusting to age and gender Darker pixels reflect higher correlation values. Note that while the rhythm measures tended to be only mildly correlated with the clinical measures, the consistency measures were generally more strongly correlated with the clinical measures.

Figure 22

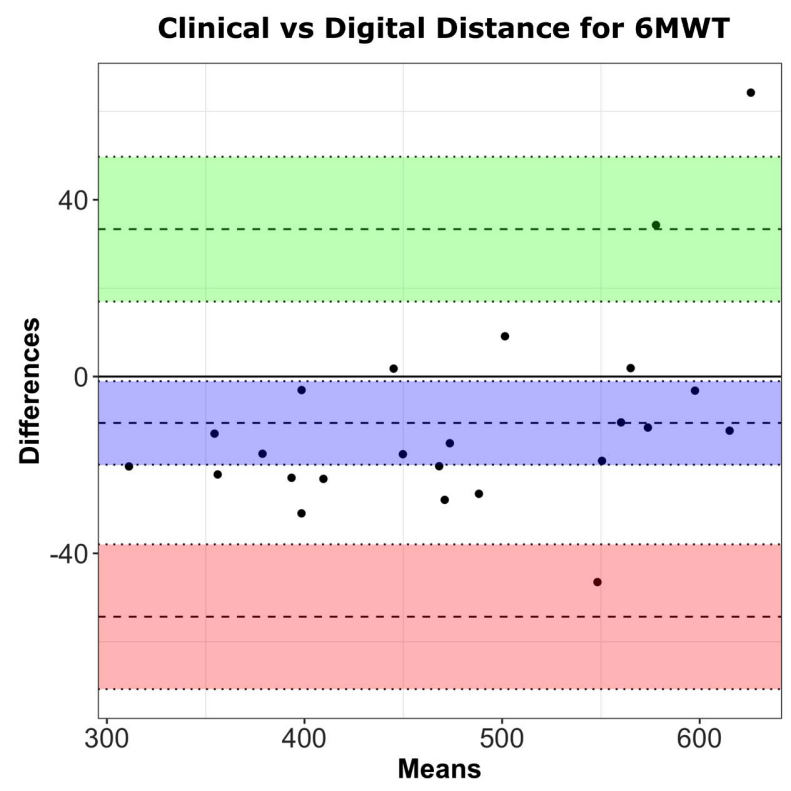

Figure 23 

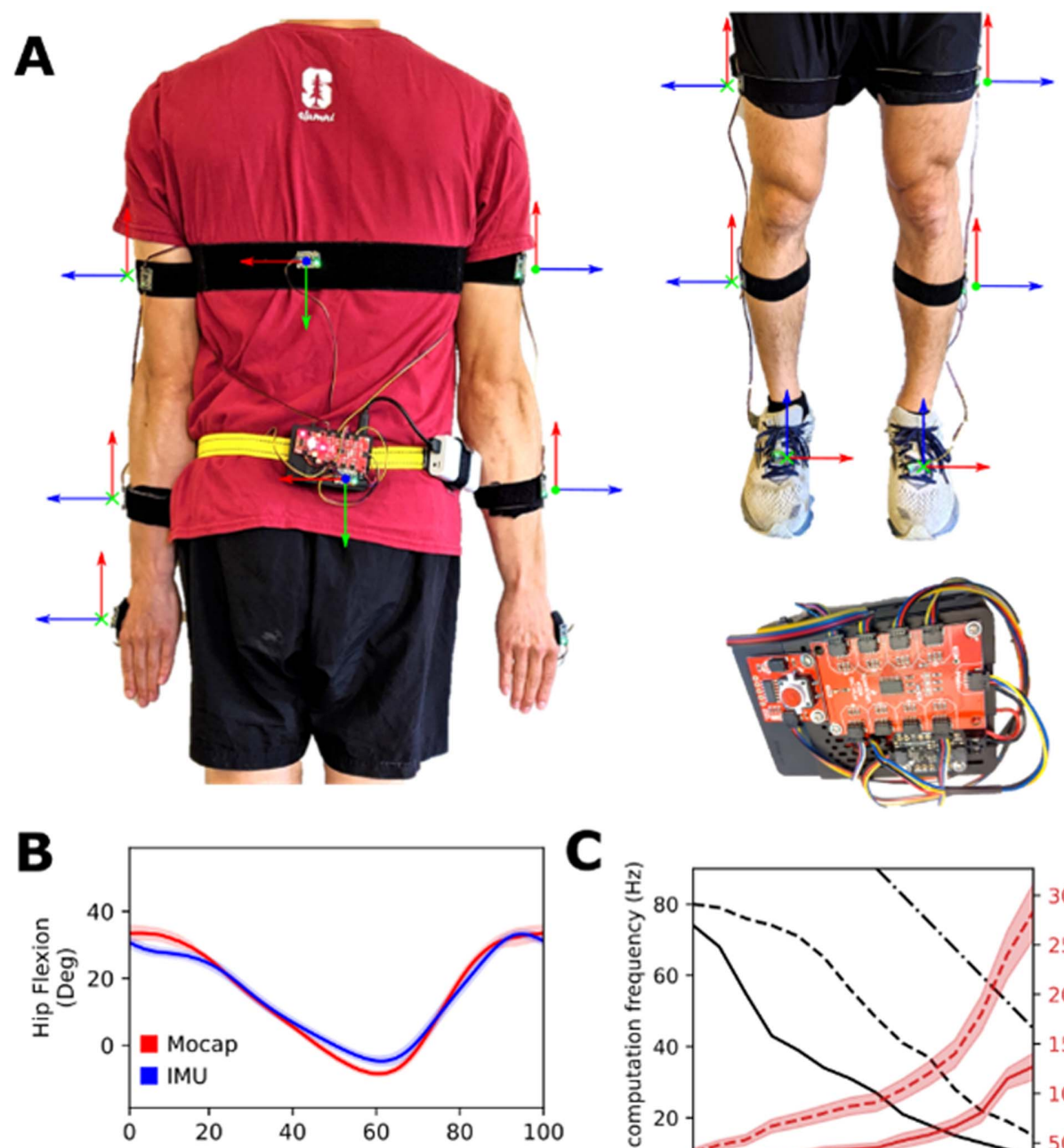

C
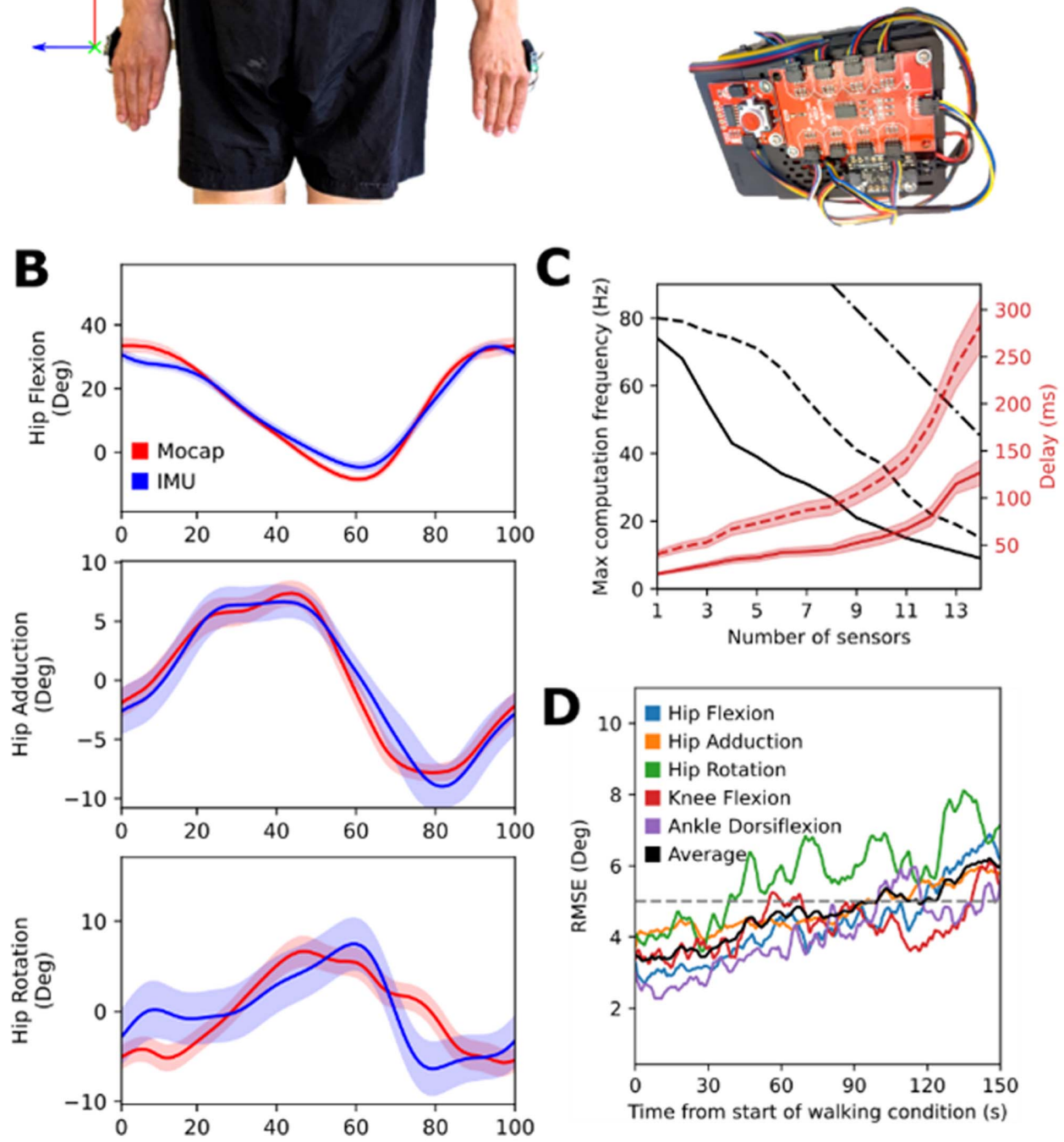

Figure 24 


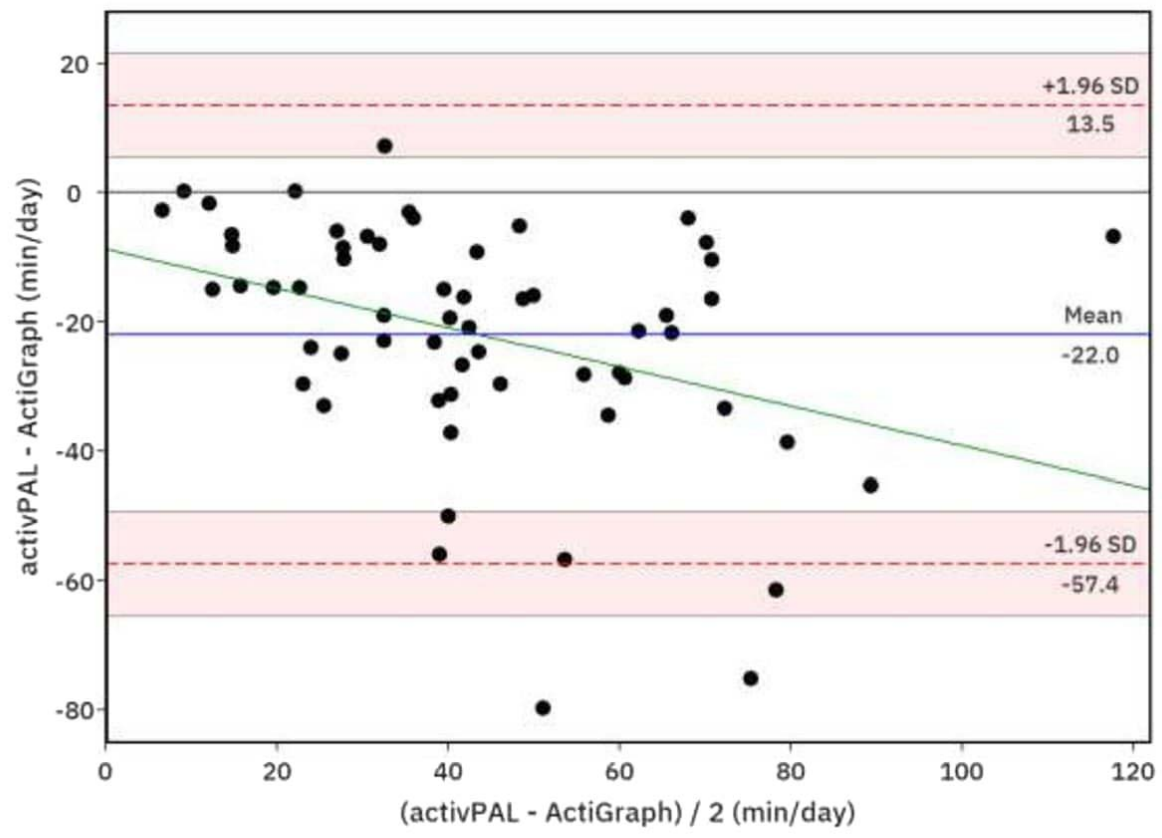

Figure 1: Bland-Altman diagram for the MVPA (mins/day) measured by activPAL4 and ActiGraph. The blue line shows the mean difference, the red dashed lines show the limits of agreement at the $95 \%$ level with the respective confidence intervals (red shaded bands), and the green line shows the linear regression trend.

Figure 25

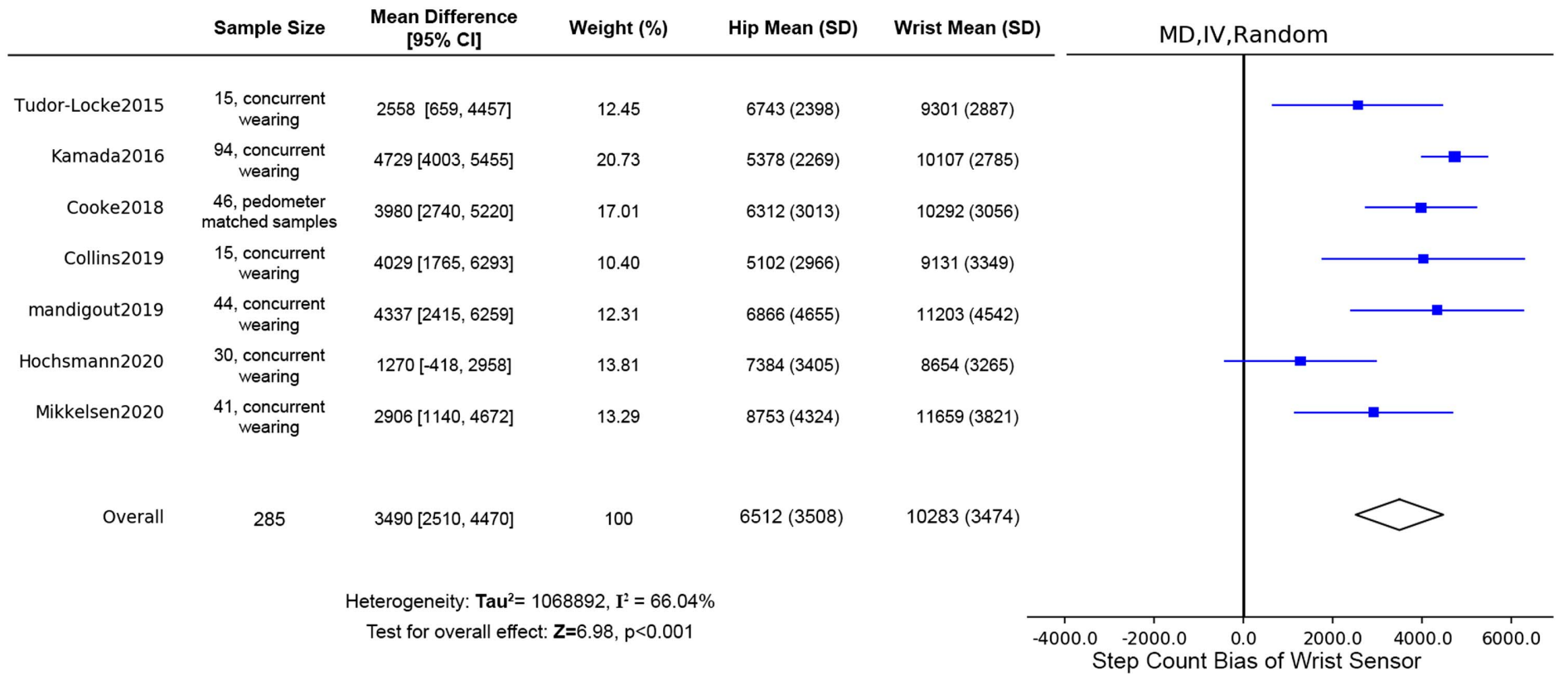

Figure 26 
Figure 27
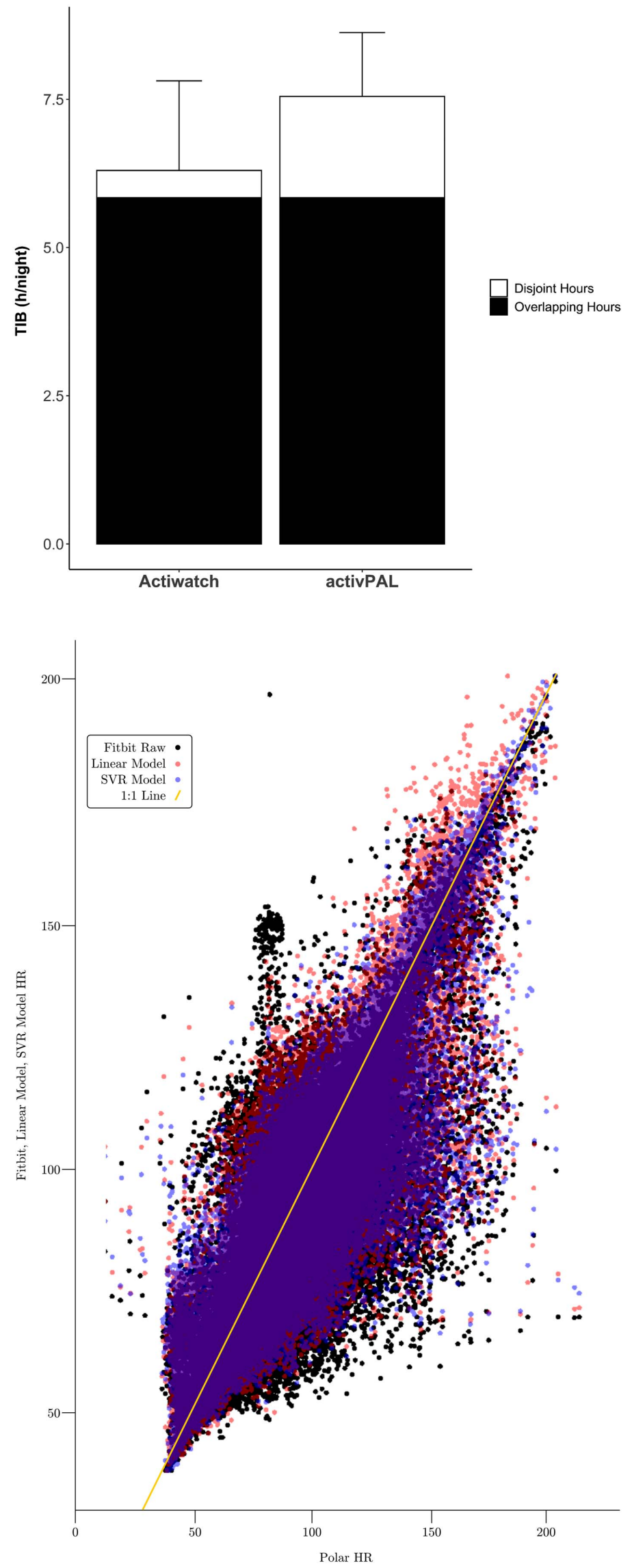

Figure 28 

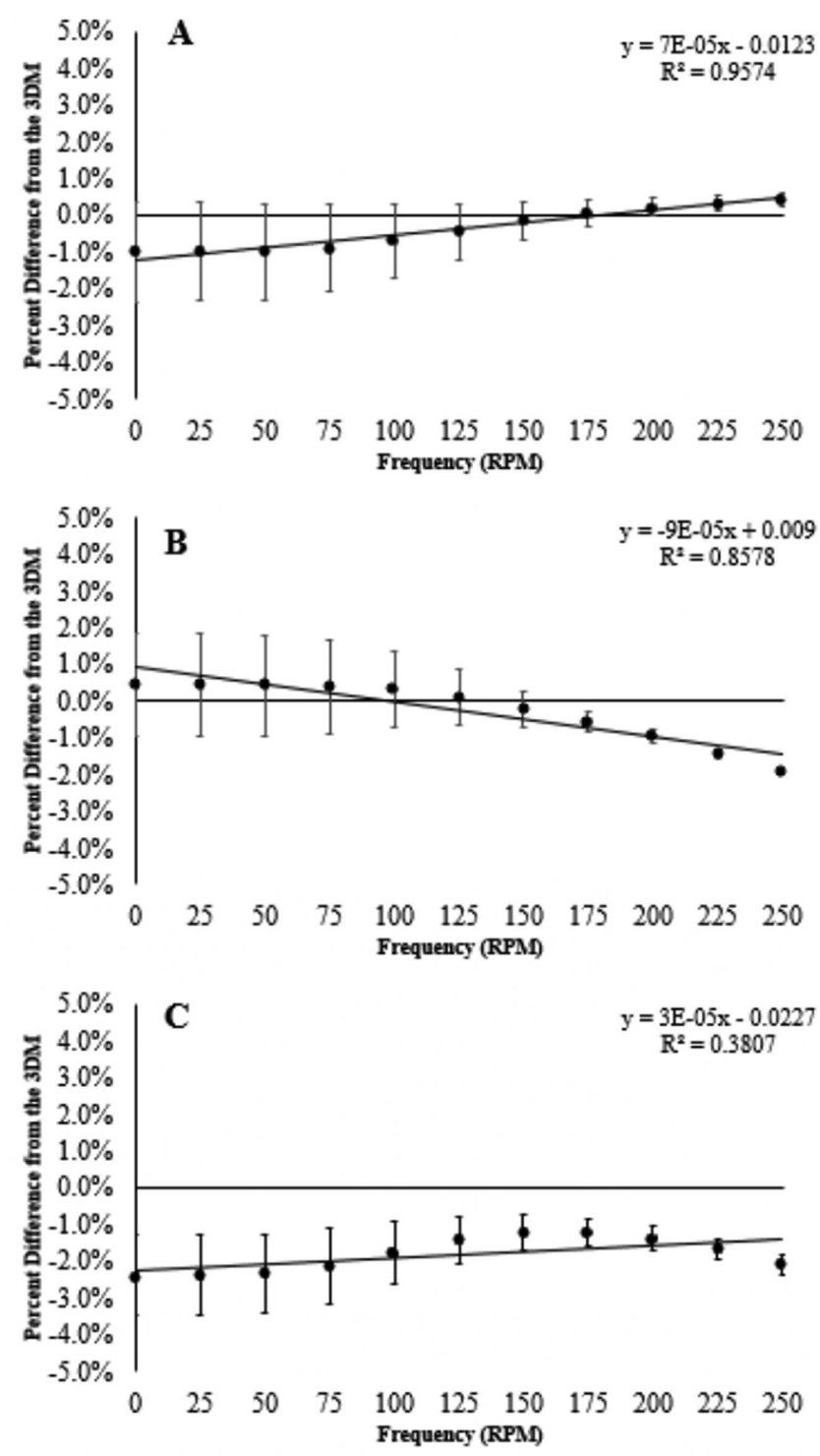

Figure 1. Percent difference (Mean $\pm S D$ ) from the $3 D M$ criterion accelerometer across frequency by device type A) CentrePoint Insight Watch, B) wGT3X-BT, and C) GT9X Link

Figure 29 


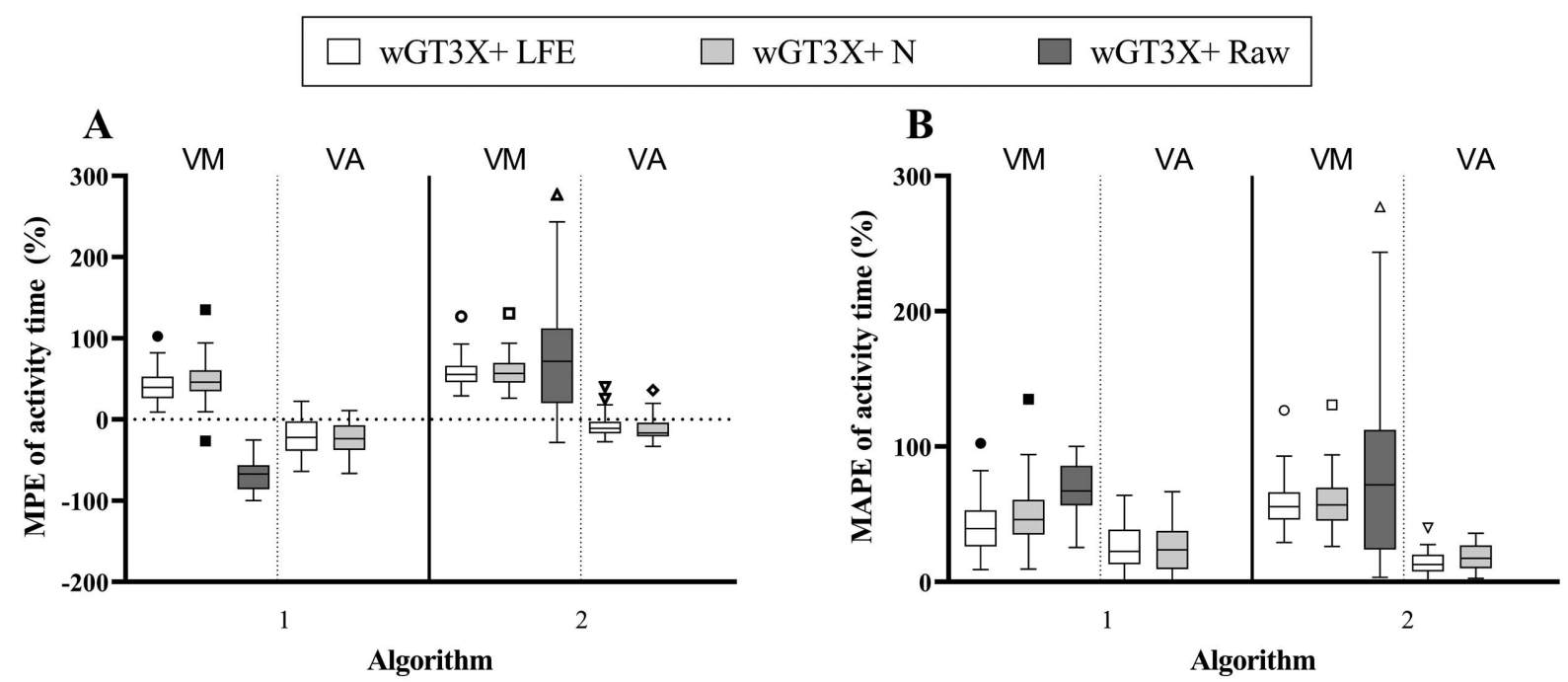

Figure 1. Median percent errors (MPE; A) and median absolute percent errors (MAPE; B) of the activity time between the wGT3X+ data (raw and counts/s with the low-frequency extension filter enabled or not) and the ActivPAL classification data. Algorithms 1 and 2 represent the computation of the threshold respectively from the total 7-day data and on moving windows of 1 hour.

LFE, low-frequency extension filter; $\mathrm{N}$, normal filter; VA, vertical axis; VM, vector magnitude.

Figure 30

\begin{tabular}{|l|c|c|c|c|c|c|c|}
\cline { 2 - 7 } \multicolumn{1}{c|}{} & $\begin{array}{c}\text { Mean } \\
\text { Difference } \\
{[\mathrm{s}]}\end{array}$ & $\begin{array}{c}\text { Standard } \\
\text { Deviation } \\
\text { of the } \\
\text { Difference } \\
{[\mathrm{s}]}\end{array}$ & $\begin{array}{c}\text { Minimum } \\
\text { value of the } \\
\text { Difference } \\
{[\mathrm{s}]}\end{array}$ & $\begin{array}{c}\text { Maximum } \\
\text { value of the } \\
\text { Difference } \\
{[\mathrm{s}]}\end{array}$ & $\begin{array}{c}\text { Lower Limit } \\
\text { of } \\
\text { Agreement } \\
{[\mathrm{s}]}\end{array}$ & $\begin{array}{c}\text { Upper Limit } \\
\text { of } \\
\text { Agreement } \\
{[\mathrm{s}]}\end{array}$ & $\begin{array}{c}\text { Inter-rater } \\
\text { reliability } \\
\text { ICC(3,1) }\end{array}$ \\
\hline $\begin{array}{l}\text { Total Duration of the } \\
\text { Test ( }=30)\end{array}$ & 0.00 & 0.10 & -0.14 & 0.29 & -0.19 & 0.19 & 1.00 \\
\hline $\begin{array}{l}\text { Duration of the fist Sit- } \\
\text { to-Stand ( }=30)\end{array}$ & 0.08 & 0.08 & -0.07 & 0.35 & -0.08 & 0.24 & 0.75 \\
\hline $\begin{array}{l}\text { Duration of the last } \\
\text { Stand-to-Sit (N=30) }\end{array}$ & -0.08 & 0.08 & -0.24 & 0.10 & -0.08 & 0.24 & 1.00 \\
\hline $\begin{array}{l}\text { Duration of the four } \\
\text { Sit-to-Stand (N=120) }\end{array}$ & 0.00 & 0.09 & -0.25 & 0.35 & -0.16 & 0.17 & 0.91 \\
\hline $\begin{array}{l}\text { Duration of the four } \\
\text { Stand-to-Sit ( }=120)\end{array}$ & 0.01 & 0.07 & -0.29 & 0.24 & -0.13 & 0.15 & 0.93 \\
\hline
\end{tabular}

Figure 31 

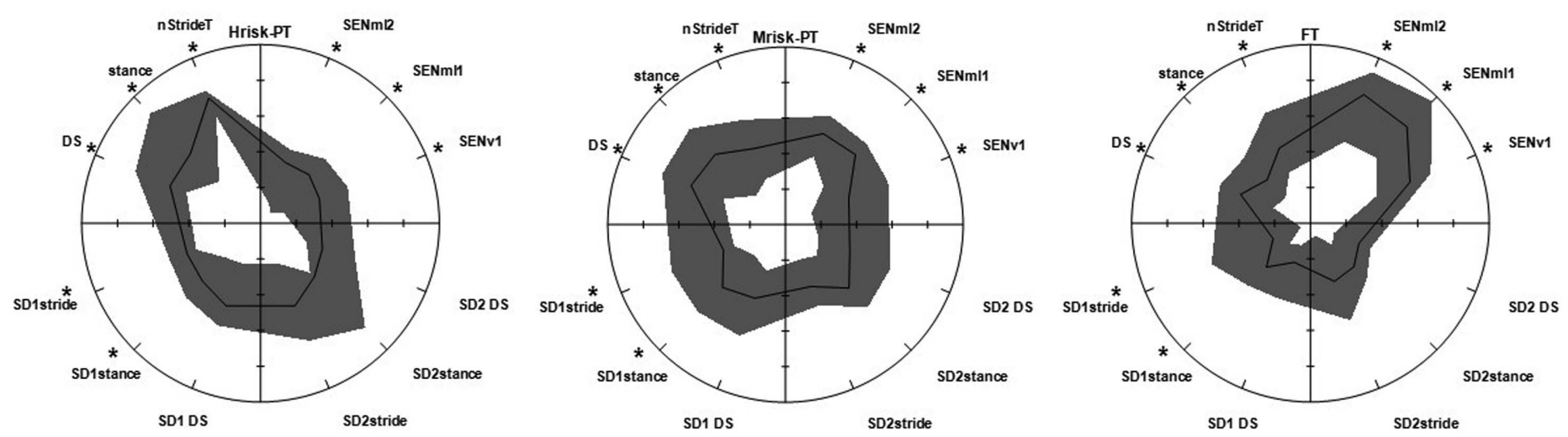

Figure 1. Polar bands (median, $25^{\text {th }}$ and $75^{\text {th }}$ percentiles) for Hrisk-PT, Mrisk-PT and FT children. Asterisks indicate significant effect of risk of motor delay $(\mathrm{p}<0.05)$. Represented parameters are:

1) 'Motor complexity': Sample entropy on the vertical $(\operatorname{SENv}(\tau=1))$ and mediolateral axis $(\operatorname{SENml}(\tau=1,2))$.

2) 'Temporal parameters': normalized stride (nStrideT), stance time (stance) and double support (DS).

3) 'Short term varibility' (SD1) for strideT, stance and DS.

4) 'Long term variability' (SD2) for strideT, stance and DS.

Figure 32

Figure 33

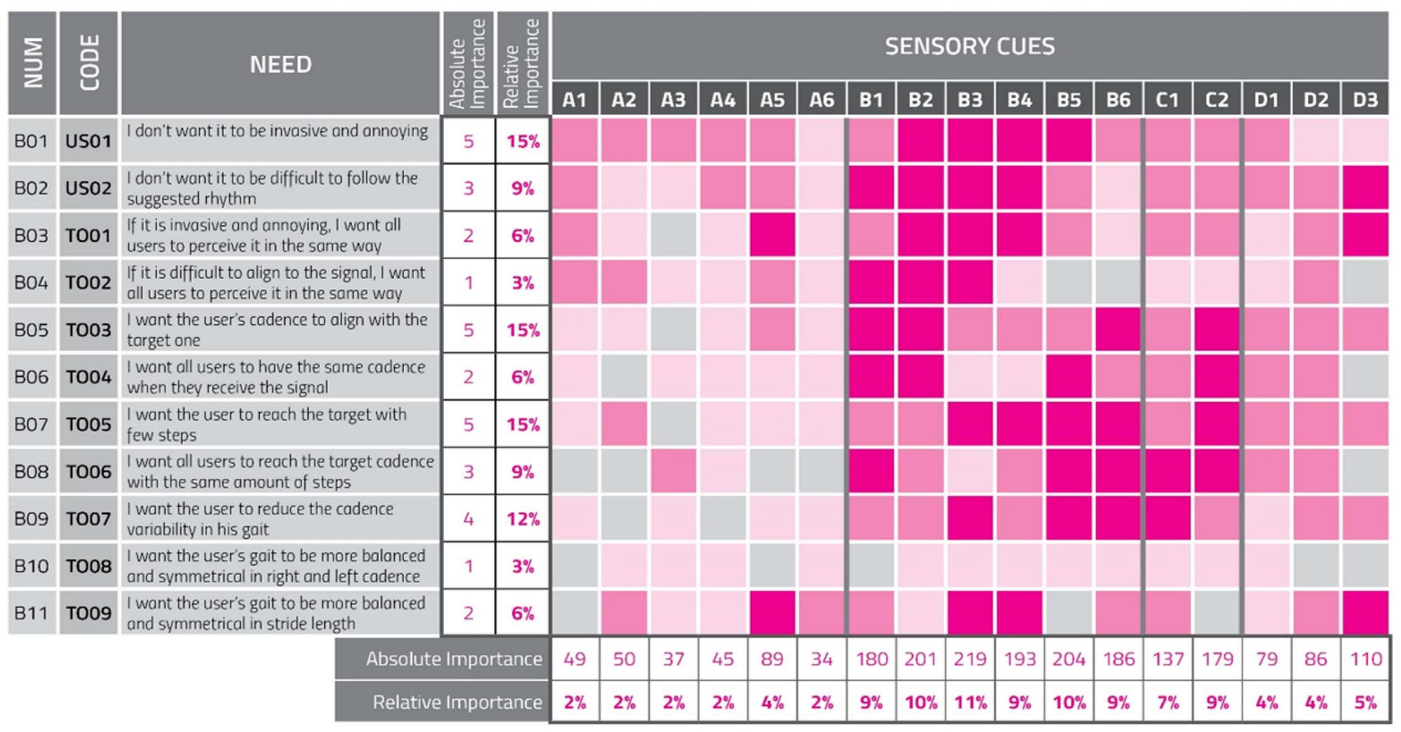

Fig. 1. Quality-Function-Deployment (QFD) matrix. A1:A6 Visual cues; B1:B6 Auditory cues; C1:C2 Haptic cues; D1:D3 Multimodal cues. 


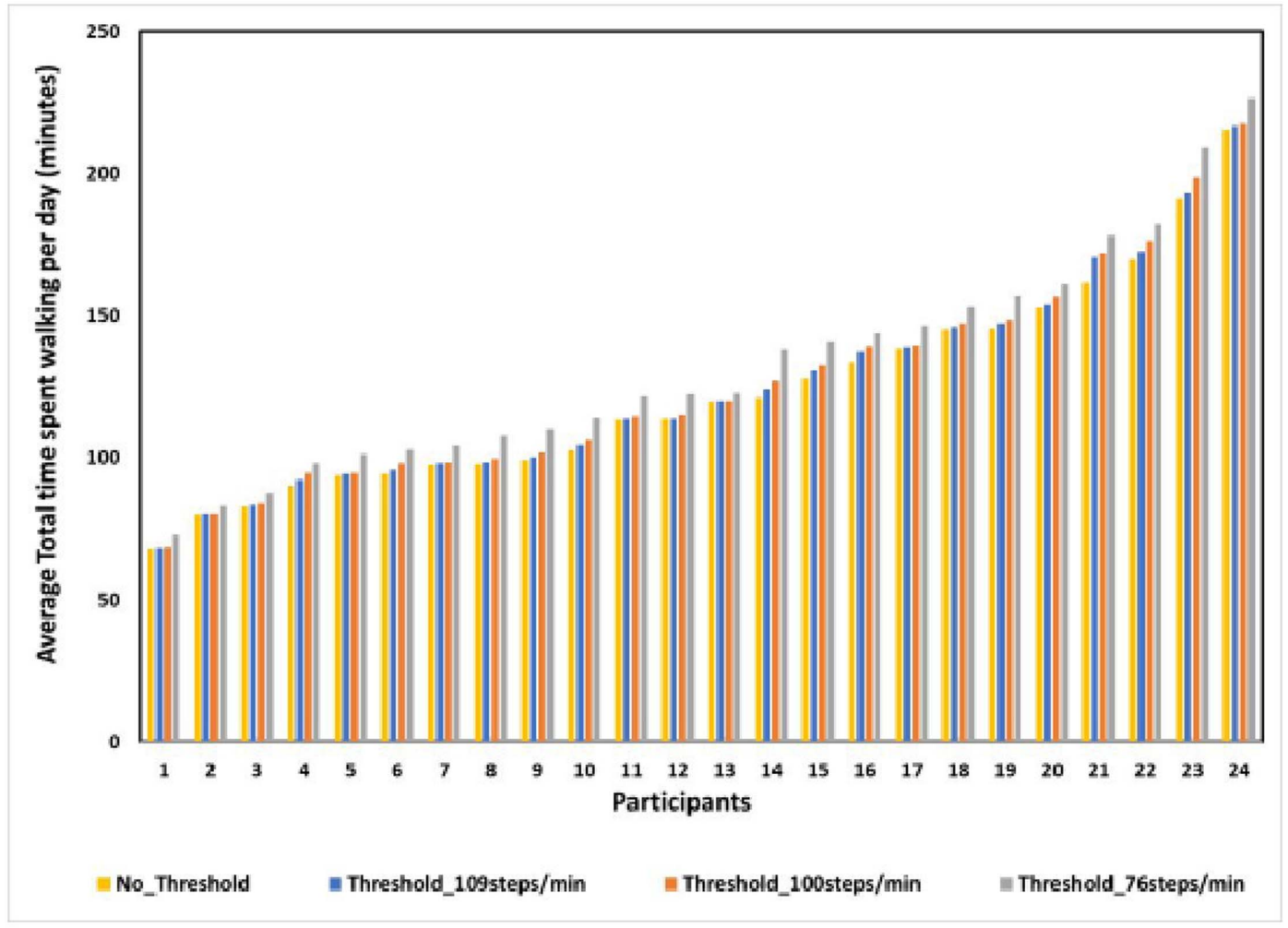

Figure 1: Average total time spent walking per day at different cadence thresholds for moderate-to-vigorous physical activity

Figure 34

Differences in accelerometer measured patterns of physical activity and sleep/rest between ethnic groups across different ages: an analysis of UK Biobank

Nathan P. Dawkins, MSc ${ }^{1,2}$ (ORCID: 0000-0002-6374-7908), Tom Yates, PhD ${ }^{1,2}$ (ORICD: 0000-0002-5724-5178), Cameron Razieh, MSc ${ }^{1,2}$ (ORCID: 0000-0003-3597-2945), Charlotte L. Edwardson, PhD ${ }^{1,2}$ (ORICD: 0000-0001-6485-9330), Ben Maylor, PhD ${ }^{1,2}$ (ORICD: 00000002-4503-0479), Francesco Zaccardi, PhD ${ }^{1,3}$ (ORCID: 0000-0002-2636-6487), Kamlesh Khunti, MD ${ }^{1,4}$ (ORICD: 0000-0003-2343-7099), Alex V. Rowlands, PhD ${ }^{1,2,5}$ (ORCID: 0000-0002-1463-697X).

1. Diabetes Research Centre, University of Leicester, Leicester General Hospital, Leicester, UK

2. NIHR Leicester Biomedical Research Centre, UK

3. Leicester Real World Evidence Unit, Leicester Diabetes Centre, Leicester General Hospital, Leicester, UK

4. NIHR Applied Research Collaboration East Midlands, Leicester General Hospital, UK

5. Alliance for Research in Exercise, Nutrition and Activity (ARENA), Sansom Institute for Health Research, Division of Health Sciences, University of South Australia, Adelaide, Australia

Corresponding author: Nathan Dawkins, Diabetes Research Centre, University of Leicester,

Leicester General Hospital, Leicester, LE5 4PW, UK. Nd177@le.ac.uk. Tel: +44 116258

4322

Figure 35 
Table 1

Odds ratios and 95\% confidence intervals of sexual orientation, demographic covariates, and muscle-strengthening activities $\geq 2$ days/week

\begin{tabular}{|c|c|c|c|c|}
\hline Variable & OR & $95 \%$ CI OR & AOR & $95 \% \mathrm{CI} A O R$ \\
\hline \multicolumn{5}{|l|}{ Sexual Orientation } \\
\hline Heterosexual & Referent & Referent & Referent & Referent \\
\hline Lesbian & 1.54 & 1.33 to 1.78 & 1.39 & 1.20 to 1.61 \\
\hline Bisexual & 1.30 & 1.13 to 1.50 & 1.15 & 0.99 to 1.34 \\
\hline Other & 1.01 & 0.74 to 1.38 & 0.89 & 0.63 to 1.25 \\
\hline \multicolumn{5}{|l|}{ Race/Ethnicity } \\
\hline Non-Hispanic White & Referent & Referent & Referent & Referent \\
\hline Non-Hispanic Black & 0.63 & 0.60 to 0.68 & 0.76 & 0.71 to 0.82 \\
\hline Hispanic & 0.66 & 0.62 to 0.71 & 0.80 & 0.75 to 0.86 \\
\hline Other & 0.75 & 0.69 to 0.81 & 0.62 & 0.57 to 0.67 \\
\hline \multicolumn{5}{|l|}{ Body Mass Index Classification } \\
\hline Normal & Referent & Referent & Referent & Referent \\
\hline Underweight & 0.63 & 0.55 to 0.71 & 0.66 & 0.58 to 0.75 \\
\hline Overweight & 0.66 & 0.63 to 0.69 & 0.73 & 0.69 to 0.76 \\
\hline Obese & 0.44 & 0.42 to 0.46 & 0.49 & 0.47 to 0.52 \\
\hline \multicolumn{5}{|l|}{ Marital Status } \\
\hline Married & Referent & Referent & Referent & Referent \\
\hline Divorced/Widowed/Separated & 0.74 & 0.71 to 0.77 & 0.96 & 0.92 to 1.01 \\
\hline Never Married & 1.11 & 1.06 to 1.17 & 1.09 & 1.04 to 1.15 \\
\hline \multicolumn{5}{|l|}{ Poverty } \\
\hline Yes & 0.55 & 0.51 to 0.59 & 0.66 & 0.62 to 0.71 \\
\hline No & Referent & Referent & Referent & Referent \\
\hline \multicolumn{5}{|l|}{ Education } \\
\hline Less than high school & 0.25 & 0.23 to 0.28 & 0.35 & 0.32 to 0.38 \\
\hline High school/GED & 0.42 & 0.40 to 0.44 & 0.49 & 0.46 to 0.51 \\
\hline Some college/college degree & Referent & Referent & Referent & Referent \\
\hline \multicolumn{5}{|l|}{ 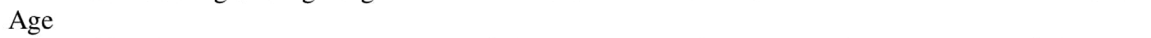 } \\
\hline 18 to 24 & Referent & Referent & Referent & Referent \\
\hline 25 to 34 & 0.94 & 0.87 to 1.02 & 0.90 & 0.83 to 0.98 \\
\hline 35 to 44 & 0.82 & 0.75 to 0.89 & 0.83 & 0.76 to 0.91 \\
\hline 45 to 54 & 0.74 & 0.68 to 0.81 & 0.77 & 0.70 to 0.85 \\
\hline 55 to 64 & 0.66 & 0.61 to 0.72 & 0.70 & 0.64 to 0.77 \\
\hline $65+$ & 0.51 & 0.47 to 0.56 & 0.58 & 0.53 to 0.63 \\
\hline
\end{tabular}

Figure 36

Table 1.

Classification Confusion Matrix of True Values vs Predicted Values Predicted Label

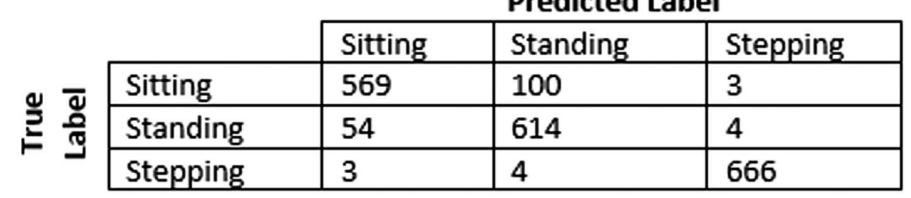

Figure 37 
General Model

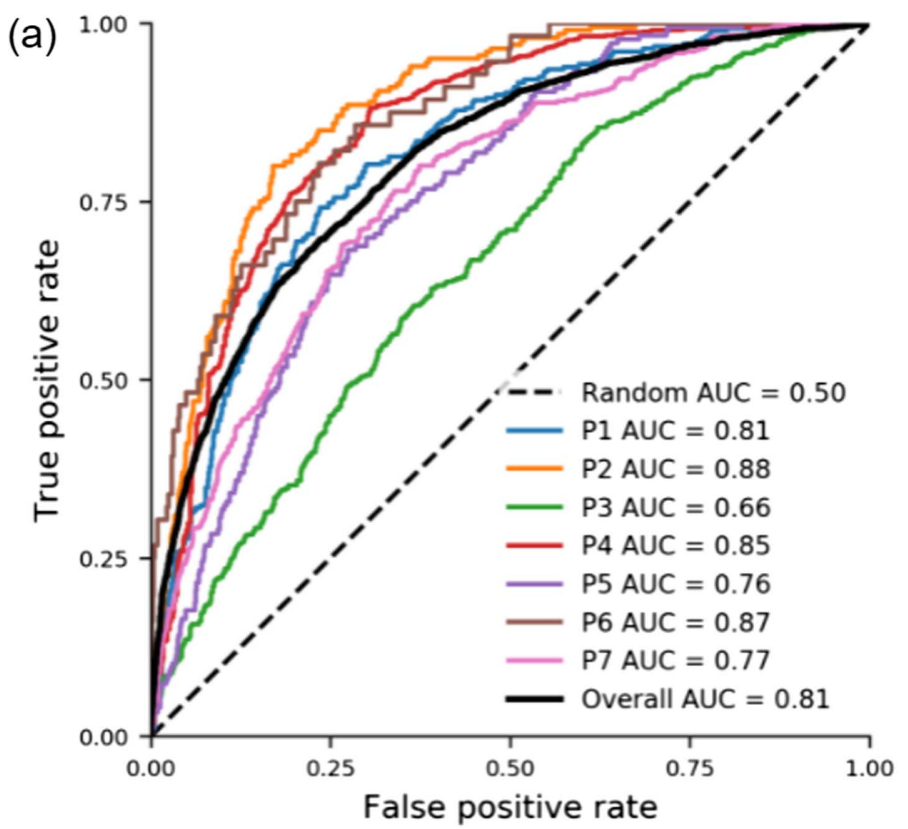

Participant-Specific Model

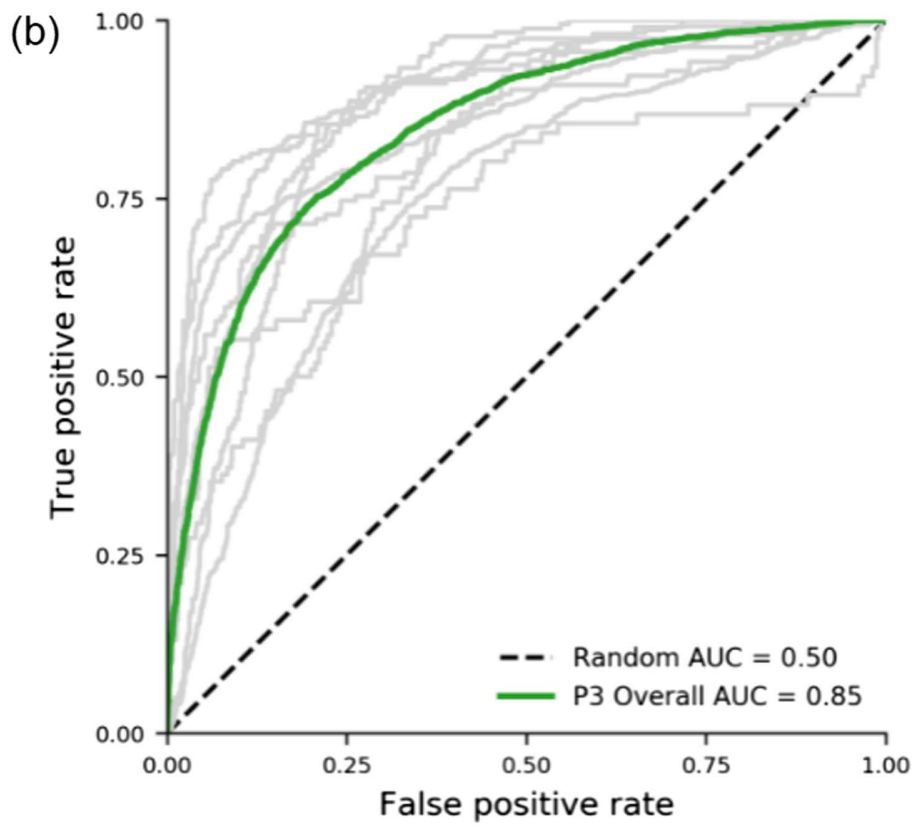

(a) Areas under the curves (AUCs) of the receiver operator characteristics for the general model, trained with leave-one-subject-out cross-validation, demonstrate good performance. (b) The participant-specific model, trained with leave-one-walk-out cross-validation (each grey line representing a single walk), improves AUC from 0.66 to 0.85 for the worst-performing participant in the general model (P3).

Figure 38

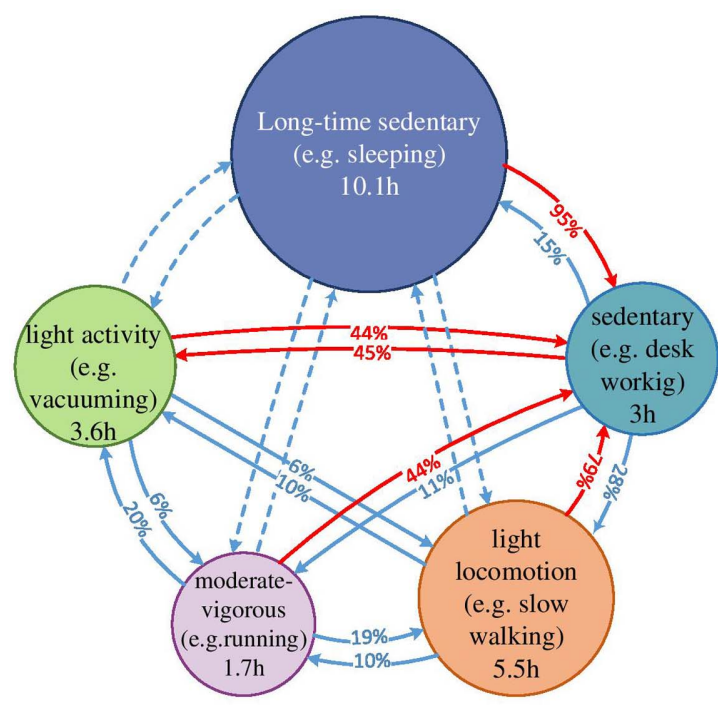

a. CLBP-

Transition smaller than $5 \%$

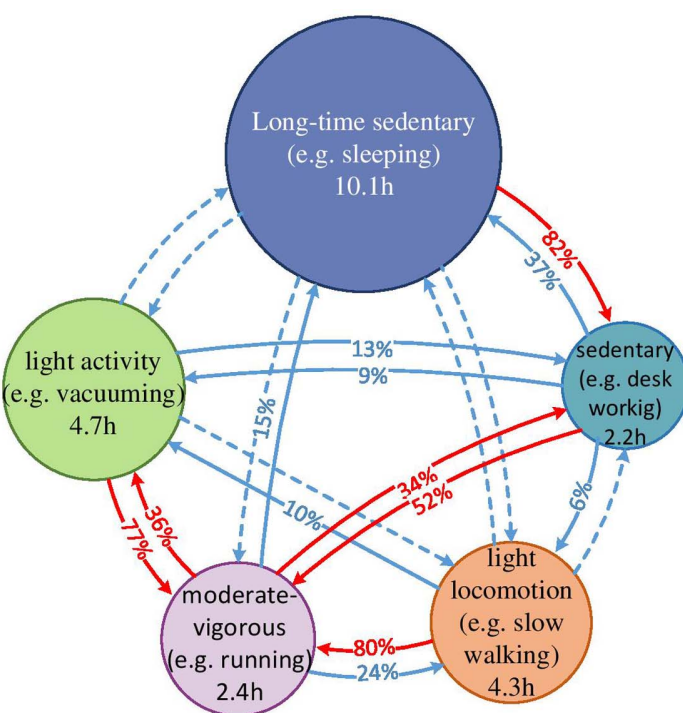

b. CLBP+

Figure. the 5 state HSMM for CLBP- and CLBP+

Figure 39 


\begin{tabular}{|r|c|}
\multicolumn{2}{|c|}{ Accelerometer data } \\
& GOTO_Inclusion \\
\hline $\mathrm{N}$ & 154 \\
\hline Sex (male \%) & $49.4 \%$ \\
\hline Age (years) & $62.8(5.8)$ \\
\hline Length (m) & $1.72(0.1)$ \\
\hline Weight(kg) & $79.2(9.9)$ \\
\hline BMI & $26.8(2.5)$ \\
\hline
\end{tabular}
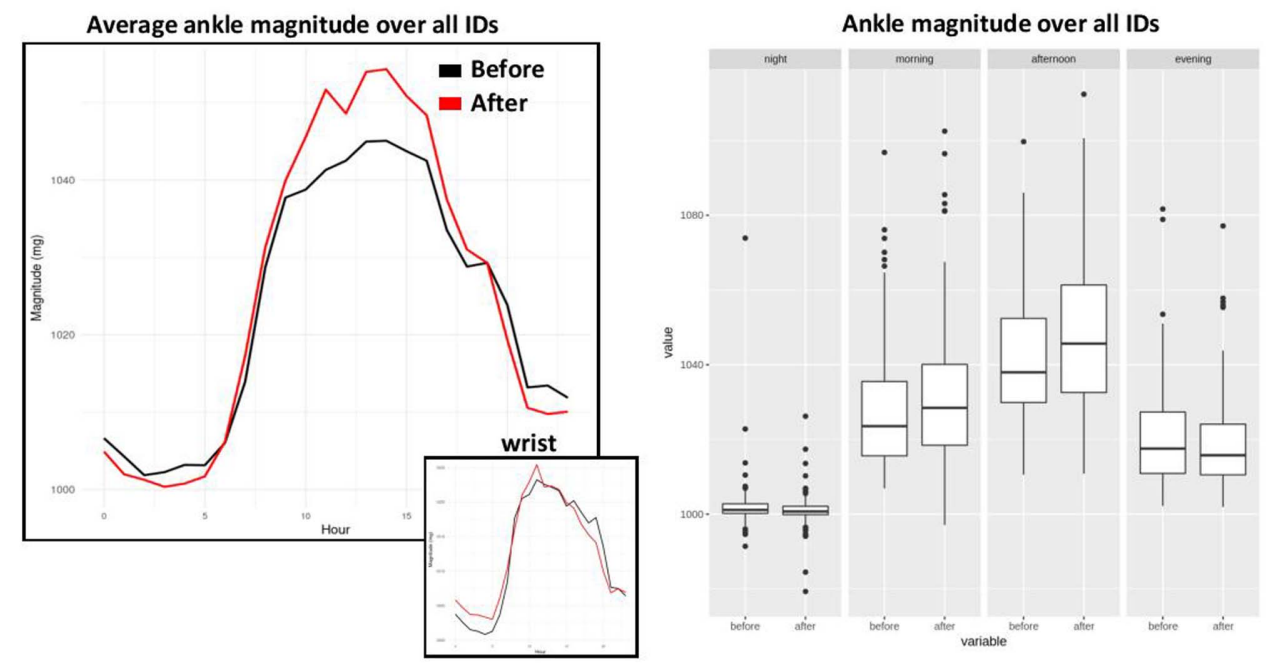

Figure 40 


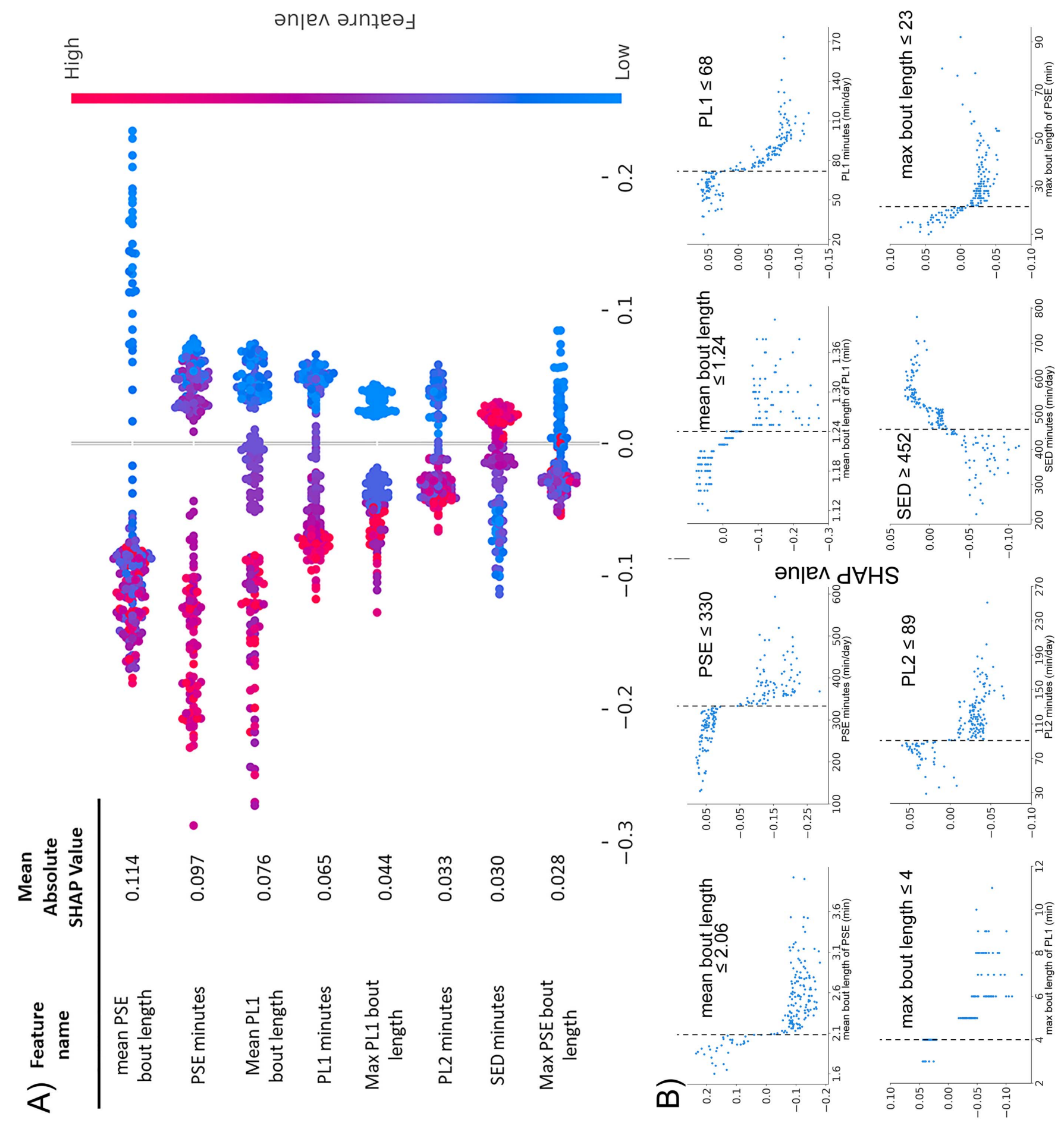

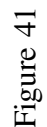


ACC

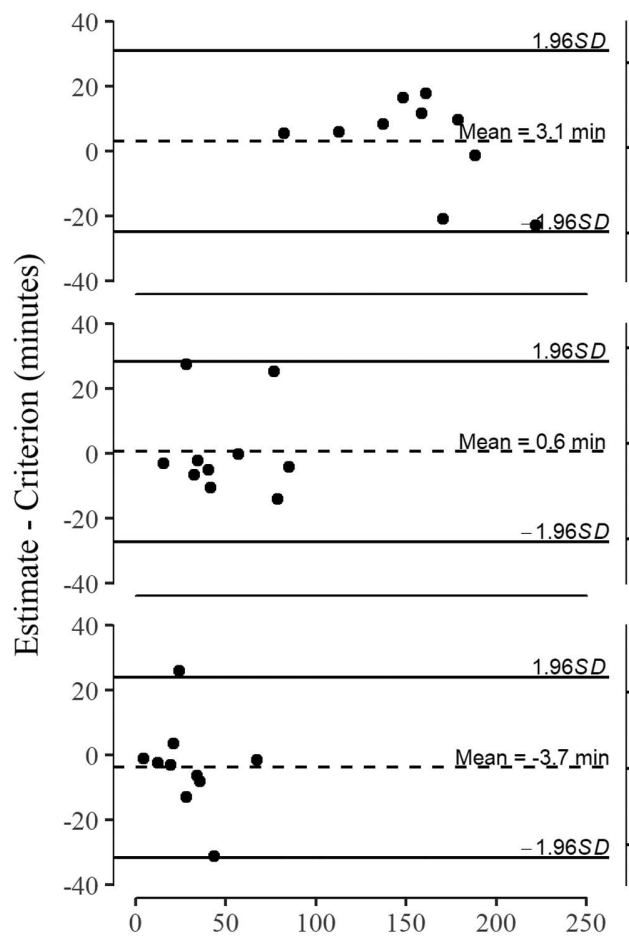

GYRO
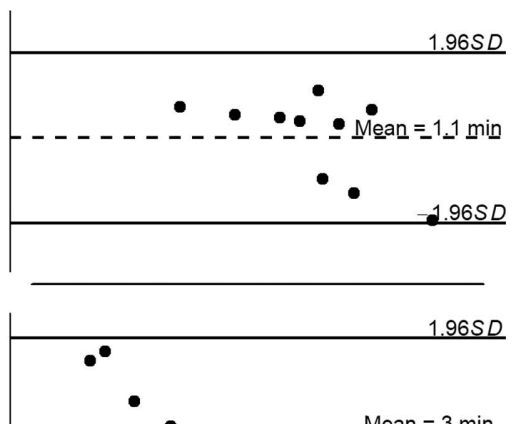

$-\bullet--e^{-}------$Mean $\equiv \underline{3} \min$
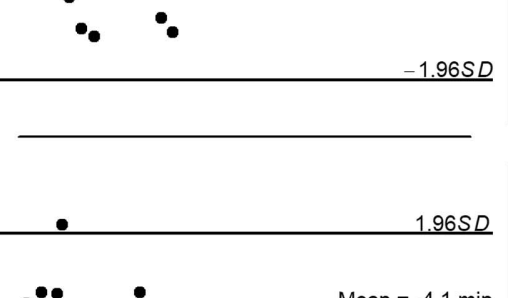

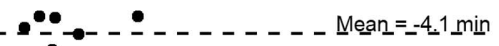
$\because \bullet$

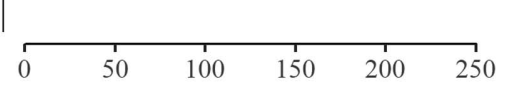

Direct Observation (minutes)
ACC+GYRO

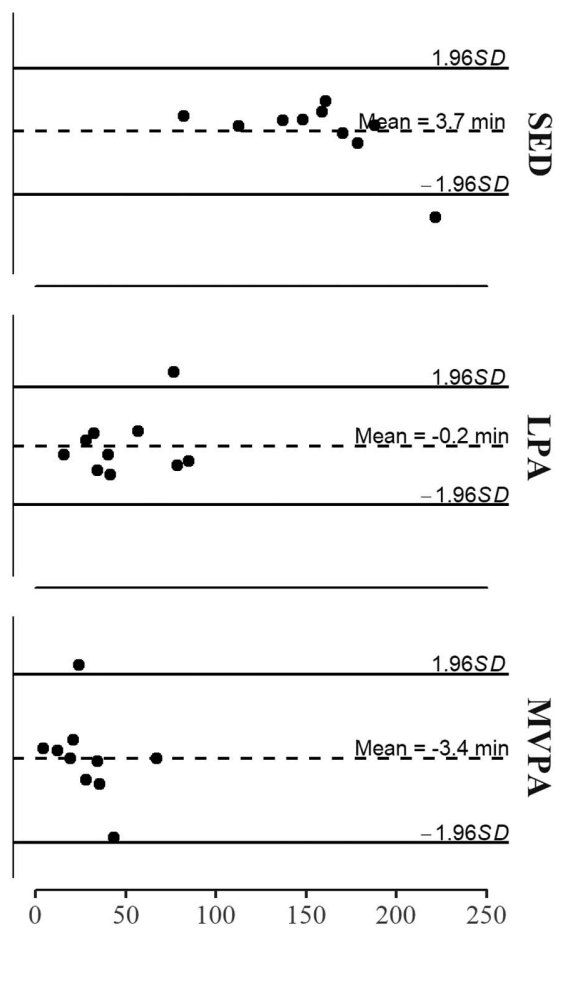

Figure 42 


\begin{tabular}{|c|c|c|c|c|}
\hline & Variable & $\begin{array}{l}\text { Subjects with } \\
\text { mild FOG during } \\
\text { daily-living* }\end{array}$ & $\begin{array}{c}\text { Subjects with } \\
\text { moderate FOG during } \\
\text { daily-living }\end{array}$ & p-value \\
\hline \multirow{6}{*}{$\begin{array}{c}\text { Subject } \\
\text { characteristics and } \\
\text { disease severity }\end{array}$} & Age (yrs) & $70(62-75.25)$ & $71(66.75-72.75)$ & 0.710 \\
\hline & Gender ( $\%$ female) & $14.29 \%$ & $14.29 \%$ & 1 \\
\hline & Disease duration (yrs) & $9.5(3.5-21.25)$ & $13.5(9.5-16)$ & 0.928 \\
\hline & MDS-UPDRS part III OFF & $47(35-52)$ & $44(34-53)$ & 0.678 \\
\hline & MDS-UPDRS III OFF item 3.11. FOG & $1(0-2)$ & $2(1-3)$ & 0.010 \\
\hline & LEDD (mg) & $546(288-1035)$ & $887(801-1235)$ & 0.018 \\
\hline \multirow{2}{*}{$\begin{array}{l}\text { Unsupervised } \\
\text { home monitoring }\end{array}$} & $\%$ TF during monitoring & $0.58(0.49-0.67)$ & $2.83(1.78-3.69)$ & 0.000 \\
\hline & Steps per day & $4684(3863-7845)$ & $9496(4425-14739)$ & 0.097 \\
\hline \multirow{14}{*}{$\begin{array}{l}\text { Amount of FOG } \\
\text { during Supervised } \\
\text { FOG-provoking } \\
\text { test (\%TF) }\end{array}$} & 4-meter walk OFF & $0.0(0.0-0.0)$ & $1.89(0.0-20.64)$ & 0.046 \\
\hline & TUG single task OFF & $0(0-3.87)$ & $5.08(0-20.83)$ & 0.066 \\
\hline & TUG with dual-task OFF & $0.0(0.0-1.14)$ & $6.99(0.0-41.86)$ & 0.012 \\
\hline & Hotspot door task OFF & $0(0-10.18)$ & $21.64(1.3-34.8)$ & 0.009 \\
\hline & Hotspot personal task OFF & $7.076(0-21.68$ & $27.98(8.74-39.48)$ & 0.113 \\
\hline & Turning task single task OFF & $21.94(4.39-62.69)$ & $21,17(5.33-60.56)$ & 0.949 \\
\hline & Turning task with dual-task OFF & $25.12(0-58.93)$ & $27.25(3.39-80.63)$ & 0.611 \\
\hline & 4-meter walk ON & $0(0-0)$ & $0(0-0)$ & 0.317 \\
\hline & TUG single task ON & $0(0-1.50)$ & $0(0-0)$ & 0.308 \\
\hline & TUG with dual task ON & $0(0-0)$ & $0(0-0.62)$ & 0.630 \\
\hline & Hotspot door task ON & $0(0-1.47)$ & $0(0-0)$ & 0.442 \\
\hline & Hotspot personal task ON & $1.33(0-7.40)$ & $0(0-4.27)$ & 0.401 \\
\hline & Turning task single task ON & $9.88(0-60.16)$ & $0(0-39.77)$ & 0.210 \\
\hline & Turning task with dual task ON & $9.29(0-57.51)$ & $4.41(0-40.98)$ & 0.320 \\
\hline \multirow{4}{*}{$\begin{array}{l}\text { Time to complete } \\
\text { FOG-provoking } \\
\text { tests (sec) }\end{array}$} & 4-meter walk OFF & $3.96(3.57-4.95)$ & $4.88(4.49-5.96)$ & 0.233 \\
\hline & TUG with dual-task OFF & $16.4(13.9-19.6)$ & $23.6(13.8-54.4)$ & 0.041 \\
\hline & Hotspot door task OFF & $16(15-16)$ & $24(17-27)$ & 0.774 \\
\hline & Turning task with dual-task OFF & $82(46-82)$ & $62(45-131)$ & 0.192 \\
\hline \multirow{2}{*}{$\begin{array}{c}\text { Self-report } \\
\text { Questionnaires }\end{array}$} & New FOG Questionnaire** & $21(19-24.25)$ & $30(22.25-37.75)$ & 0.510 \\
\hline & Characterising FOG Questionnaire*** & $19.5(15-27.7)$ & $23(19.7-25.3)$ & 0.510 \\
\hline
\end{tabular}

TF: \% time frozen; MDS-UPDRS: Movement Disorders Society-Unified Parkinson's Disease Rating Scale; TUG: Timed Up and Go; OFF: off medication state; LEDD: levodopa equivalent daily dose. Mann Whitney $U$ tests were used to compare the two groups since many variables were not normally distributed.

*Table entries are median ( $25 \%-75 \%$ percentiles)

** A Nieuwboer et al. (2009)

***E Martens et al. (2018)

Figure 43 


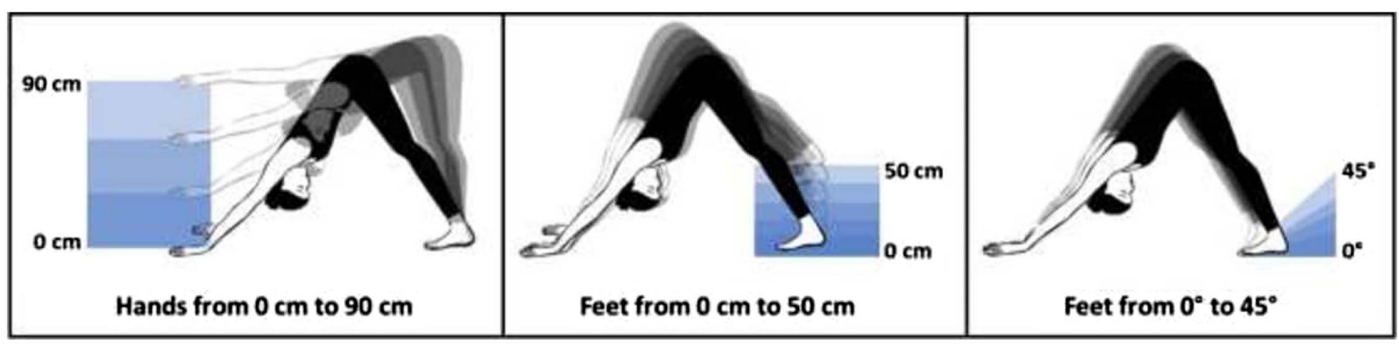

Figure 1 - Yoga position variants in the 3 section. Left: section 1, first position is with hands and feet on the floor (original) and last position with hands at $90 \mathrm{~cm}$ height; middle: section 2, first position is original and with hands on the floor and feet at $50 \mathrm{~cm}$ height; right: first position is original and last is with feet inclination of $45^{\circ}$.

Figure 44

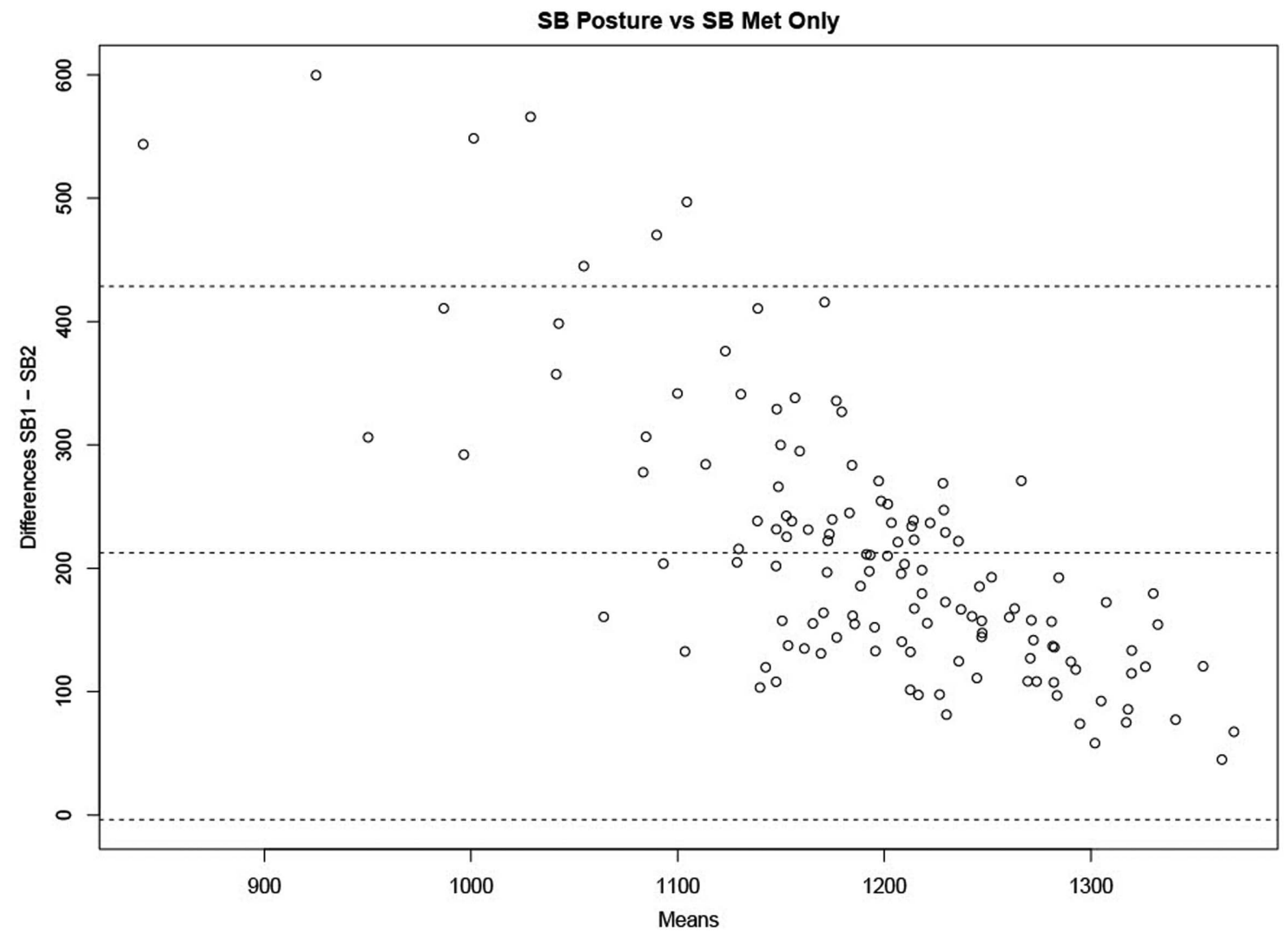

Figure 45 
Figure 1. Illustration of how a school classroom is coded using the Geosocial Observation Method (from the Geosocial Observation Method Codebook).

\section{School Classroom}

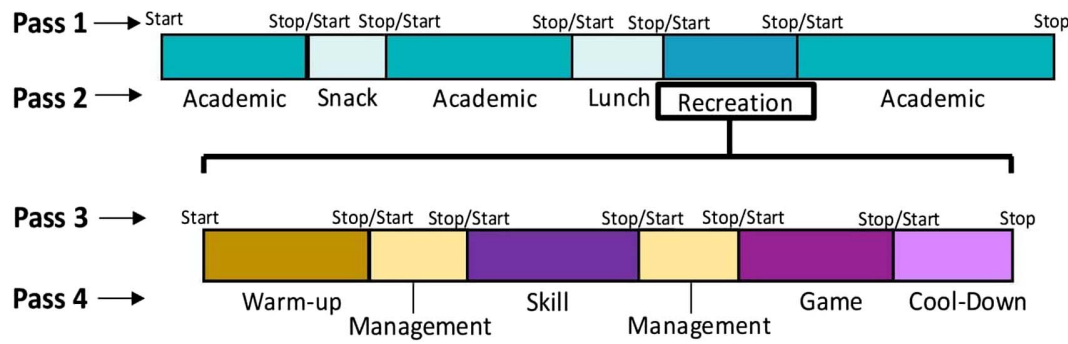

Research reported in this publication was supported by the National Cancer Institute of the National Institutes of Health under Award Number R01CA215420. The content is solely the responsibility of the authors and does not necessarily represent the official views of the National Institutes of Health.

Figure 46

Table 1. List of DMOs and corresponding statistical analyses/performance metrics. The types of DMOs for which the same 5 statistical analyses are evaluated, are matched by colour (blue, orange, green, grev and vellow) according to their structure and gramularity. For each of the DMOs is detailed: Performance metrics: The performance metrics that will be used to compare Wearable Sensor based DMOs vs. DMOs of the reference system. Criterion validity: The criterion validity metrics that will be used to compare Wearable Sensor based DMOs vs. DMOs of the reference system. Plots: The tvpe ofplots listed in the table will be used to visualise distributions and validity results.

\begin{tabular}{|c|c|c|c|c|c|c|c|c|c|c|c|c|c|c|c|c|}
\hline \multirow[b]{2}{*}{ Bluck } & \multirow[b]{2}{*}{ DMO } & \multicolumn{5}{|c|}{ PLRTORMANCL MLTRICS } & \multicolumn{7}{|c|}{ CRTIRION VALIDITY } & \multicolumn{3}{|c|}{ PLOTS } \\
\hline & & 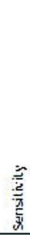 & 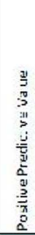 & 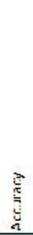 & $\frac{\sqrt{\frac{0}{7}}}{\frac{8}{8}}$ & 总 & 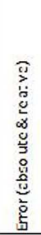 & 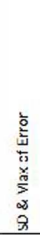 & 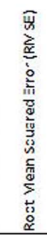 & $\frac{\overline{5}}{\frac{1}{2}}$ & 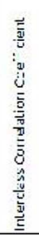 & 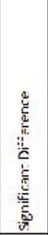 & 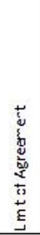 & 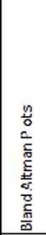 & 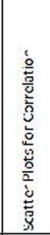 & 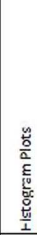 \\
\hline \multirow[t]{2}{*}{ GAIT S=OUFNCF NFTFMTION (GSD) } & $\begin{array}{l}\text { Numb=r nf Gait Serqu=nres } \\
\text { Start of Sait S=quenr:= detert=d } \\
\text { End of Gait Sezuence detectes }\end{array}$ & & & & & & & & & & & & & & & \\
\hline & Duration o- Geit Sequence detectes & & & & & & & & & & & & & & & \\
\hline STFP DFTFMTINN (SR) & $\begin{array}{c}\text { Initial C-Intect Fvents (Time) } \\
\text { Step Duration } \\
\text { Stride Duration }\end{array}$ & & & & & & & & & & & & & & & \\
\hline $\begin{array}{c}\text { IFFT/RIGHT } \\
\text { IDFNTIFICATION (I-RI) }\end{array}$ & $\begin{array}{c}\text { Iaterality } \\
\text { Sesuence. C.rder }\end{array}$ & & & & & & & & & & & & & & & \\
\hline CADENCE ESTINATICN (CAD) & Caventic= (sleps/imin) & & & & & & & & & & & & & & & \\
\hline STRIDE LNNGTH [5L] & Mean Strlde Length & & & & & & & & & & & & & & & \\
\hline RENL WNLKING SPEED (RWSS) & walking speed & & & & & & & & & & & & & & & \\
\hline \multirow{5}{*}{ IURNING DEIECIIUN (IU) } & Number of lurns & & & & & & & & & & & & & & & \\
\hline & Stert o lums & & & & & & & & & & & & & & & \\
\hline & End of I Urns & & & & & & & & & & & & & & & \\
\hline & Duration of Turns & & & & & & & & & & & & & & & \\
\hline & PMaximal Angle of Turns & & & & & & & & & & & & & & & \\
\hline HEIGHI ESIIMAIION (HE) & Elevation Change & & & & & & & & & & & & & & & \\
\hline \multirow{3}{*}{ SELONDAHR UUICUMES (SU) } & rinal Contact [vents (Tims) & & & & & & & & & & & & & & & \\
\hline & Stance lhose Lurction & & & & & & & & & & & & & & & \\
\hline & Svang lohasc Duratien & & & & & & & & & & & & & & & \\
\hline
\end{tabular}

References

1. Bonci T. et al. Sensors. 2020;20(22):6509.

2. https://www.mobilise-d.eu/

Figure 47 
Figure. Distributions of daily steps (A and B), peak 1-minute cadence (C and D), and peak 30-minute cadence (E and F) for metrics derived using data from concurrently worn activPAL micro (AP) and ActiGraph (AG) GT3X+ devices with $\mathrm{AG}$ data processed using the normal extension $\left(\mathrm{AG}_{\mathrm{NE}}\right)$ and using the low frequency extension $\left(\mathrm{AG}_{\mathrm{LFE}}\right)$. Dashed lines represent the arithmetic mean of each distribution. Adult Changes in Thought (ACT) cohort $(\mathrm{n}=953$, age $=77.0$, female $=55.8 \%, 2016)$.

(A)

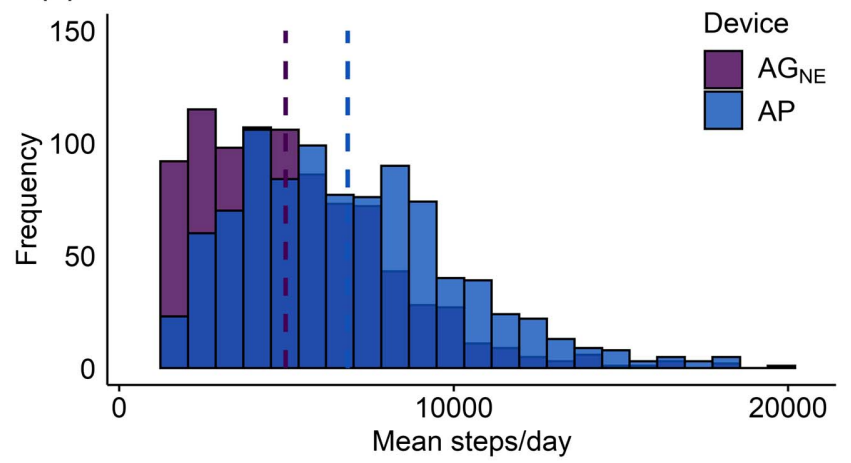

(C)

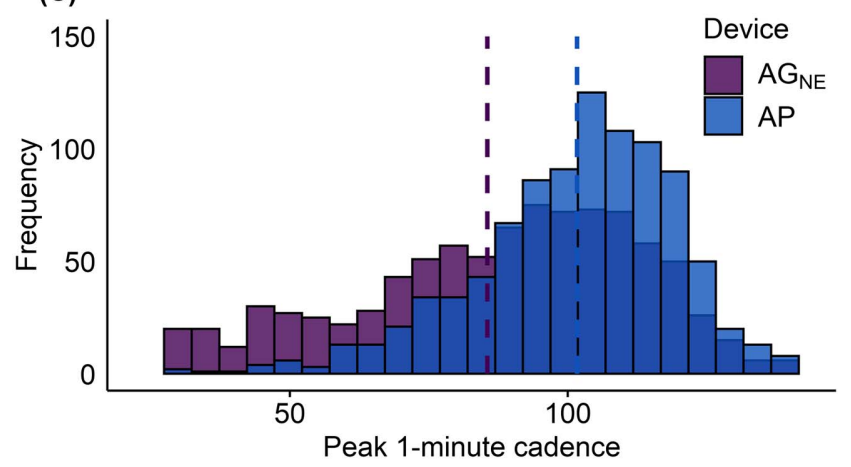

(E)

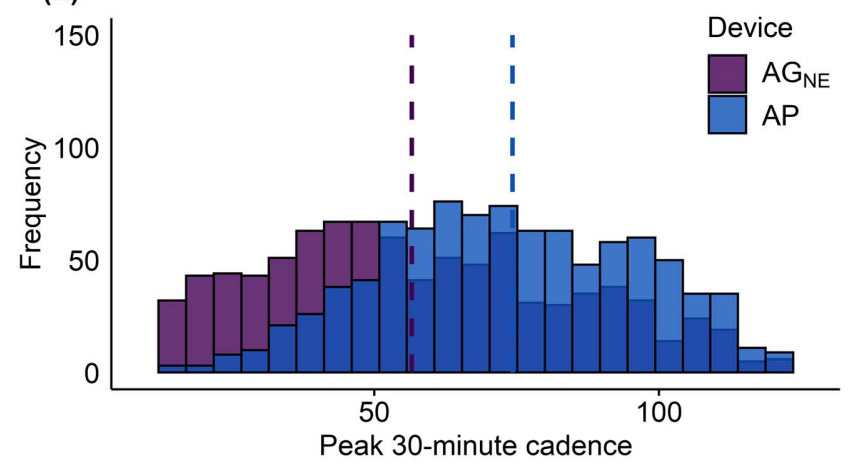

(B)

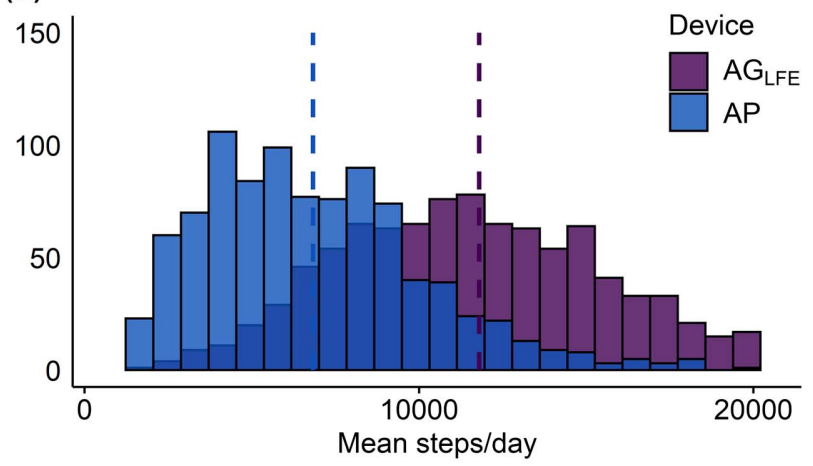

(D)

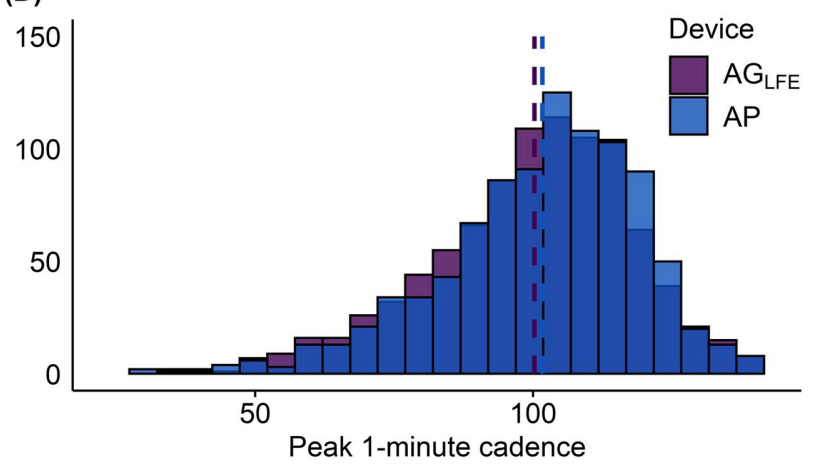

(F)

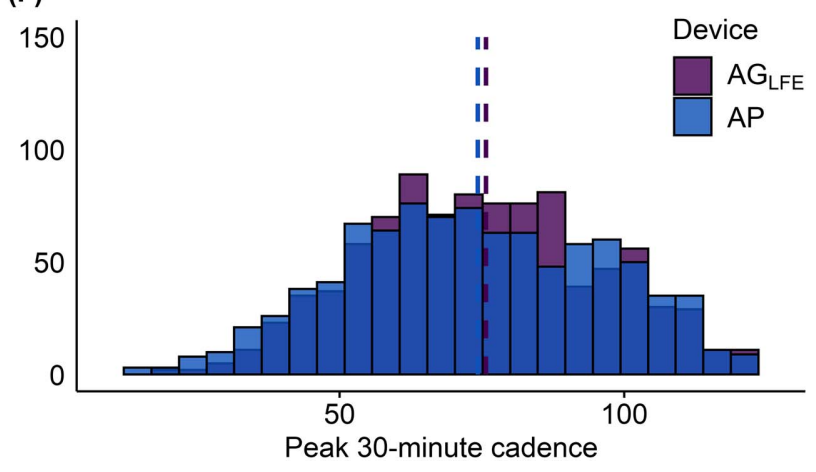

Figure 48 


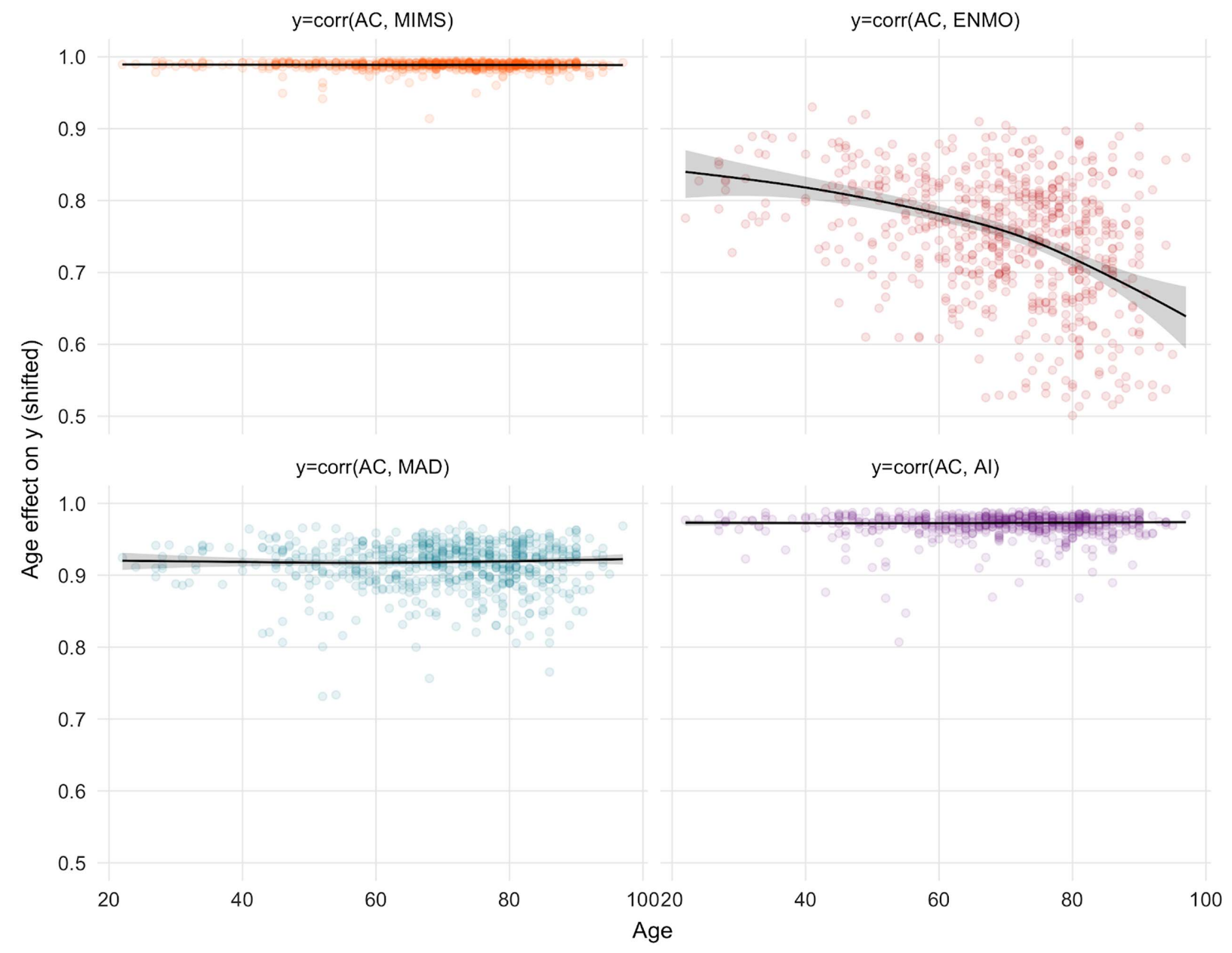

Figure 49 
(A)

Activity Level 1

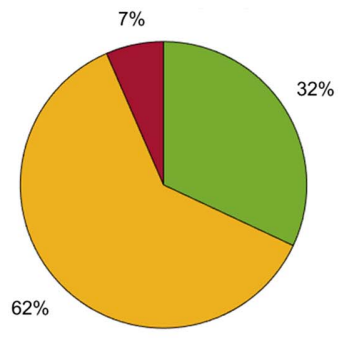

Activity Level 2

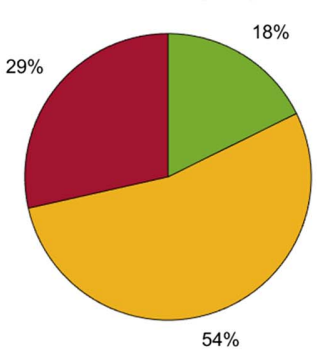

Activity Level 3

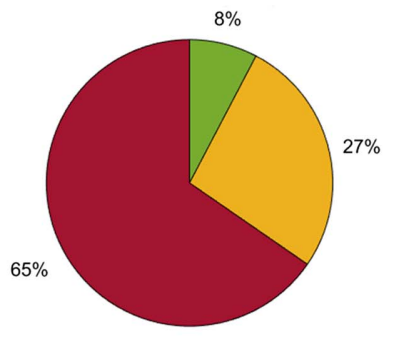

Activity Level 4

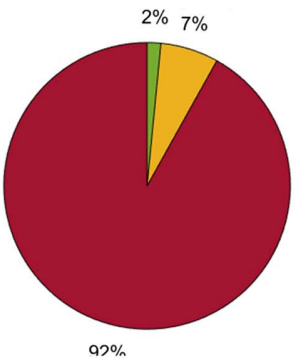

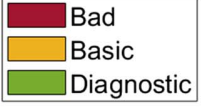

(B)

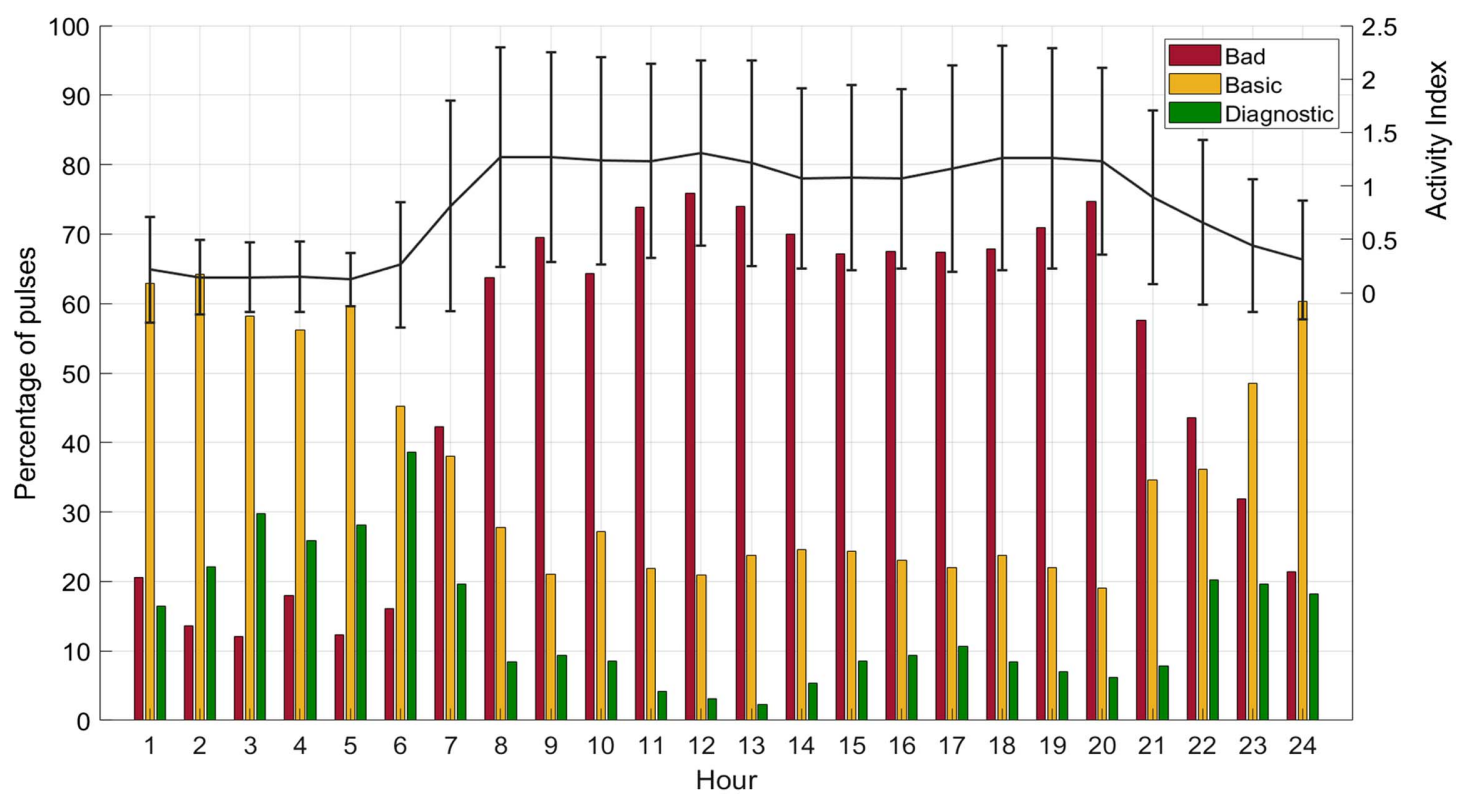

Figure 50
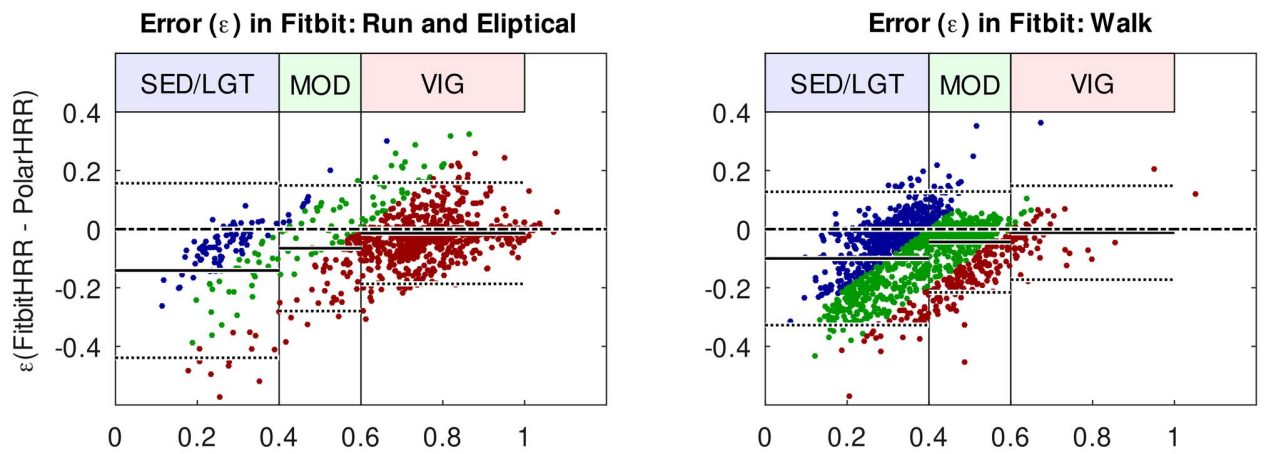

Error $(\varepsilon)$ in Fitbit: Spinning, Sport, and HIIT

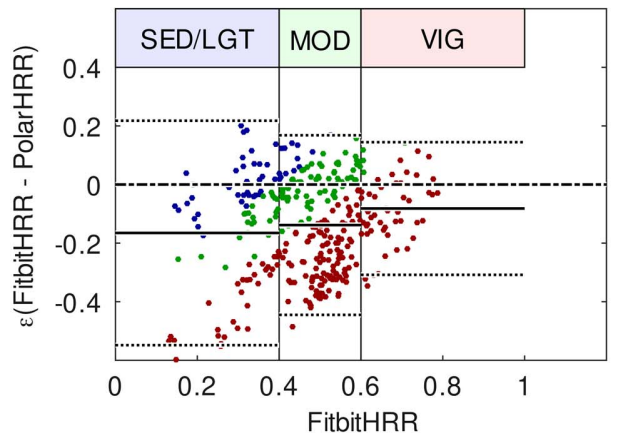

Figure 51

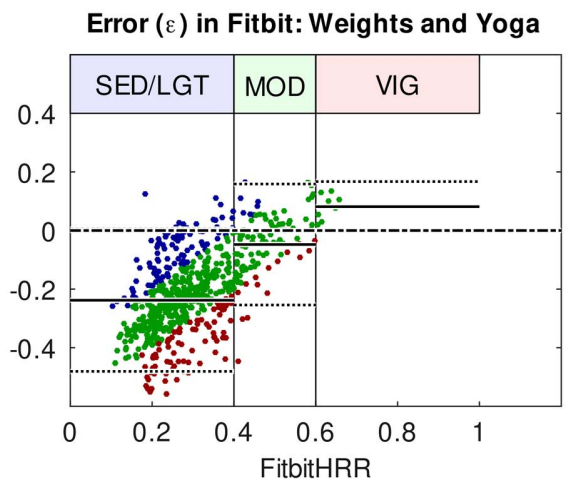




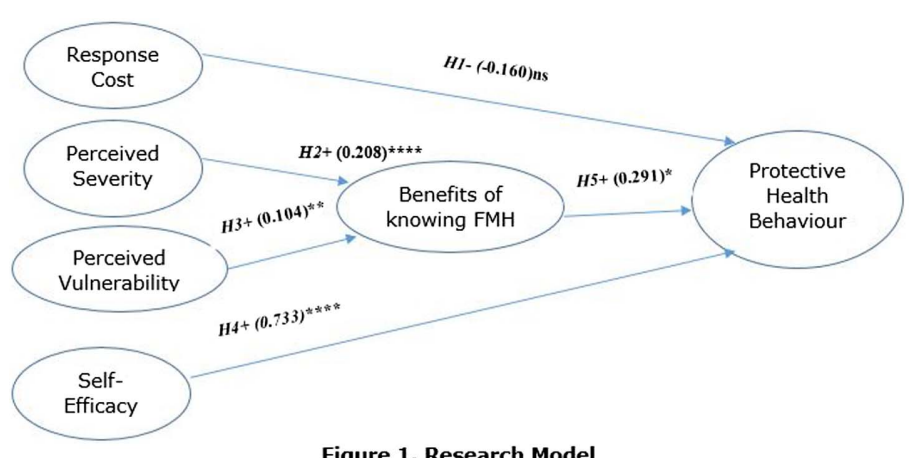

Figure 52

Figure 1. Research Model

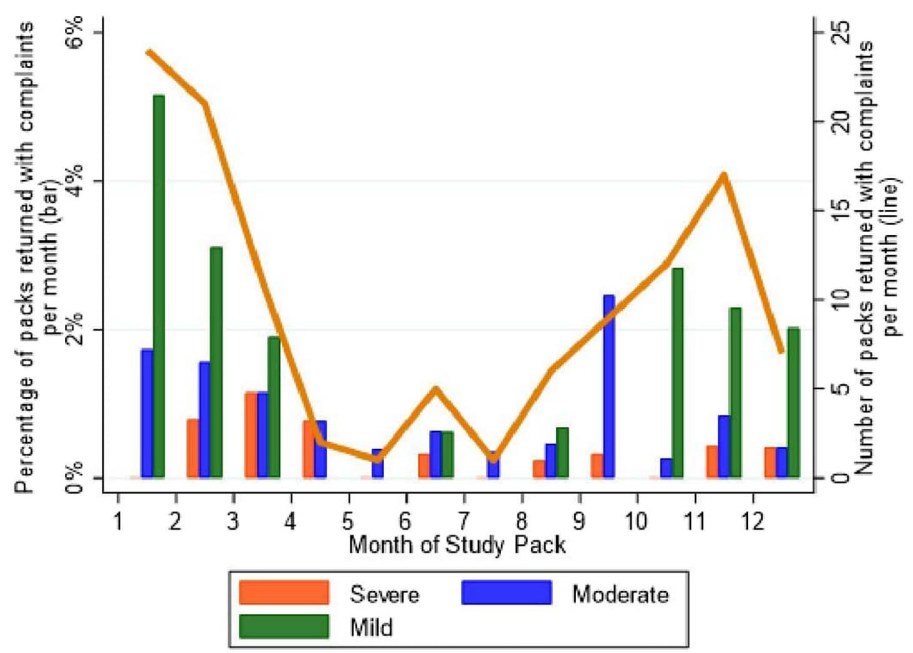

Figure 1: Frequency and severity of activPAL complaints, by month of year

Figure 53

Table 1: Ideal Distributions of Movement Behaviours for Post-Concussion Symptoms and Mean Distribution of Movement Behaviours and Post-Concussion Symptoms

\begin{tabular}{|c|c|c|c|}
\hline & \multicolumn{2}{|c|}{ Ideal Distributions } & \multirow{3}{*}{$\begin{array}{c}\text { Mean Values } \\
(n=237)\end{array}$} \\
\hline & Day 6 & Day 13 & \\
\hline & $(n=183)$ & $(n=172)$ & \\
\hline Sleep & $8.80(8.73,8.86)$ & $8.00(7.92,8.08)$ & $10.41(10.31,10.50)$ \\
\hline Stationary Time & $10.23(10.17,10.30)$ & $10.43(10.34,10.50)$ & $9.79(9.67,9.90)$ \\
\hline LPA & $3.84(3.74,3.94)$ & $4.45(4.39,4.51)$ & $3.35(3.27,3.45)$ \\
\hline MVPA & $1.12(1.10,1.14)$ & $1.12(1.09,1.14)$ & $0.45(0.41,0.49)$ \\
\hline Cognitive & $-1.89 \pm 0.15$ & $-1.51 \pm 0.34$ & $-3.78 \pm 8.28$ \\
\hline Somatic & $-1.36 \pm 0.09$ & $-1.43 \pm 0.10$ & $-6.11 \pm 6.39$ \\
\hline \multicolumn{4}{|c|}{$\begin{array}{l}\text { Movement behaviours units are in hours/day, with values representing the geometric means adjusted to } \\
\text { collectively sum to a full } 24 \text {-hours and bootstrapped } 95 \% \text { confidence intervals in parentheses; LPA = light- } \\
\text { intensity physical activity, MVPA=moderate- to vigorous-intensity physical activity; Cognitive = Change in Health } \\
\text { and Behaviour Inventory (HBI) cognitive subscale (range } 0 \text {-33) from baseline to } 2 \text {-weeks; Somatic = Change in } \\
\text { Health and Behaviour Inventory (HBI) somatic subscale (range } 0-27 \text { ) from baseline to } 2 \text {-weeks; Mean Values = } \\
\text { average across } 13 \text { days for movement behaviours (for those with } \geq 4 \text { valid days, with a valid day } \geq 10 \text { hours wear } \\
\text { time), and mean change from baseline to } 2 \text {-weeks for post-concussion symptoms. }\end{array}$} \\
\hline
\end{tabular}

Figure 54 

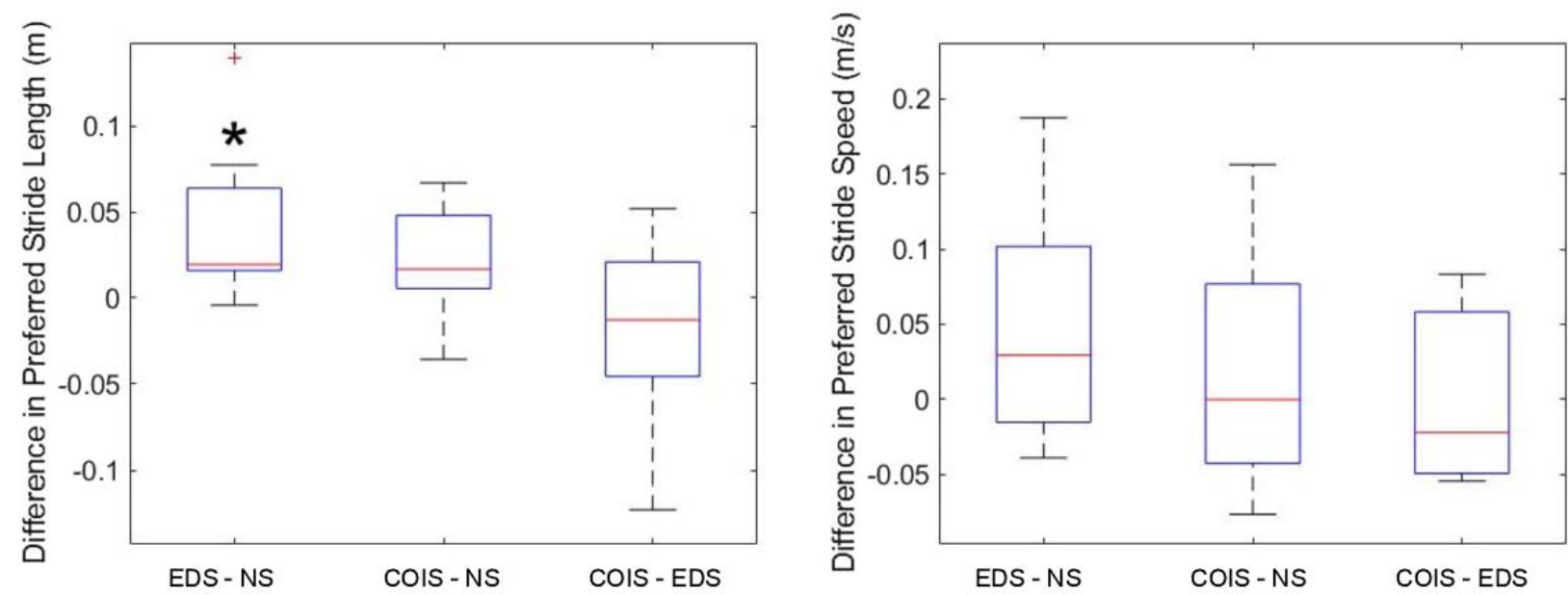

Differences in preferred stride length (left), and speed (right) for all patients. From Left to Right: extra-depth and normal shoes (EDS NS); extra-depth shoes inserted with custom insoles and normal shoes (COIS - NS); extra-depth shoes with custom insoles and extra-depth (COIS - ES). The black asterisk indicates significant differences detected by the one sample t-test (alpha=0.1) results.

Figure 55

Figure. Structural equation model for postoperative recovery after lumbar spine surgery

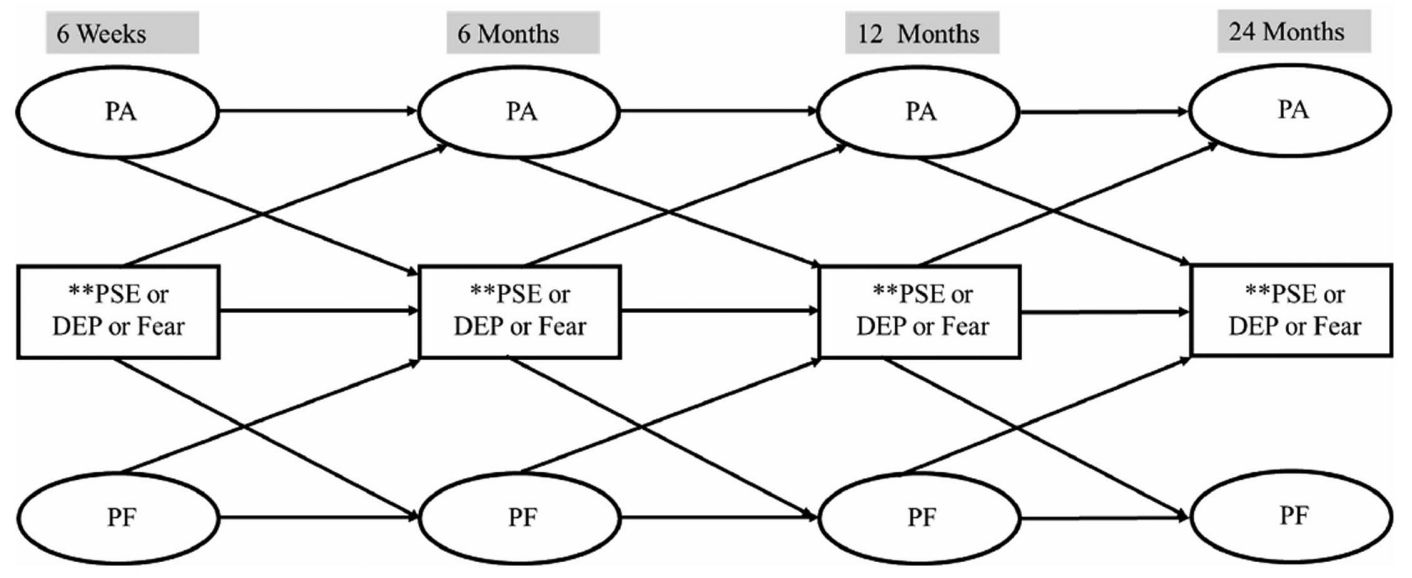

$\mathrm{PA}=$ physical activity as measured by accelerometer (average steps per day); $\mathrm{PF}=$ Physical function as measured by PROMIS; PSE = Pain self-efficacy as measured by Pain Self-Efficacy Questionnaire; Dep = Depressive symptoms as measured by Patient Health Questionnaire-9; Fear = Fear of movement as measured by 13-item Tampa Scale for Kinesiophobia.

*SEM models controlled for age, employment, comorbidities, prior spine surgery, group, and preoperative pain-self-efficacy, depression, fear of movement, physical function, and back and leg pain. **PSE, DEP and Fear were explored in separate SEM models.

Figure 56

Table 1 . Average daily steps, sitting and sleeping time in IWA and healthy populations and $\%$ difference. Average daily standing and stepping time in IWA. Data are presented as mean \pm standard deviation.

Figure 57

\begin{tabular}{|c|c|c|c|}
\hline & IWA $(n=9)$ & Healthy Populations ${ }^{*}$ & $\%$ Difference \\
\hline & Mean (SD) & Mean & \\
\hline Steps per Day & $1312(2190)$ & 5000 & -73.8 \\
\hline Daily Sitting Time (min.) & $757(356)$ & 240 & +215.4 \\
\hline Daily Sleeping Time (min.) & $525(279)$ & 480 & +9.4 \\
\hline Daily Standing Time (min.) & $112(93)$ & & \\
\hline Daily Stepping Time (min.) & $26(29)$ & & \\
\hline
\end{tabular}

IWA, individuals with aphasia; * healthy older adult population (age $\geq 60$ years) obtained from peer-reviewed journals; min. $=$ minutes. 
Table 1 . Average daily steps, sitting and sleeping time in IWA and healthy populations and $\%$ difference. Average daily standing and stepping time in IWA. Data are presented as mean \pm standard deviation.

\begin{tabular}{|c|c|c|c|}
\hline & IWA (n=9) & Healthy Populations* & \% Difference \\
\hline & Mean (SD) & Mean & \\
\hline Steps per Day & $1312(2190)$ & 5000 & -73.8 \\
\hline Daily Sitting Time (min.) & $757(356)$ & 240 & +215.4 \\
\hline Daily Sleeping Time (min.) & $525(279)$ & 480 & +9.4 \\
\hline Daily Standing Time (min.) & $112(93)$ & & \\
\hline Daily Stepping Time (min.) & $26(29)$ & & \\
\hline
\end{tabular}

Figure 58 min. $=$ minutes.

Table 1. Displays the mean and standard deviation (SD) (Mean \pm SD) of the control period (mean of Week 1 to Week 3) and the intervention period (mean of Week 4 to Week 6). The ' $p$ ' reported is the significance found via a Paired-Samples $t$-test between the control and intervention for each outcome variable. (No. $=$ Number).

\begin{tabular}{|l|c|c|c|}
\hline \multicolumn{1}{|c|}{ Outcome Variable } & Control & Intervention & $p$ \\
\hline No. of Sedentary bouts $>60$ minutes & $1.65 \pm 0.83$ & $1.41 \pm 0.90$ & 0.047 \\
\hline No. of Sedentary bouts $>90$ minutes & $0.76 \pm 0.52$ & $0.55 \pm 0.47$ & 0.017 \\
\hline Time spent sedentary $>60$ minutes & $162.01 \pm 88.79$ & $132.84 \pm 93.65$ & 0.003 \\
\hline Time spent sedentary $>90$ minutes & $96.67 \pm 71.39$ & $70.24 \pm 64.03$ & 0.011 \\
\hline
\end{tabular}

Figure 59

Table 1: PA in kids during COVID-19 compared to an age-matched pre-pandemic (historical) group

\begin{tabular}{|c|c|c|}
\hline & $\begin{array}{c}\text { During } \\
\text { COVID-19 } \\
n=33\end{array}$ & $\begin{array}{c}\text { Historical } \\
\text { Cohort } \\
n=27\end{array}$ \\
\hline \multicolumn{3}{|c|}{ Average Daily Activity Duration (mins) } \\
\hline All days & $\begin{array}{c}259 * \\
(227-291) \\
\end{array}$ & $\begin{array}{c}336 \\
(303-369) \\
\end{array}$ \\
\hline Weekdays & $\begin{array}{c}254 * \\
(226-283)\end{array}$ & $\begin{array}{c}348 \\
(315-389)\end{array}$ \\
\hline Weekend days & $\begin{array}{c}271 \\
(228-315) \\
\end{array}$ & $\begin{array}{c}301 \\
(260-342) \\
\end{array}$ \\
\hline \multicolumn{3}{|c|}{ Total Steps per Day } \\
\hline All days & $\begin{array}{c}11251 * \\
(9,998-12503)\end{array}$ & $\begin{array}{c}14,988 \\
(13,862-16,114)\end{array}$ \\
\hline Weekdays & $\begin{array}{c}11235 * \\
(9,956-12,514) \\
\end{array}$ & $\begin{array}{c}15,375 \\
(14,243-16,497) \\
\end{array}$ \\
\hline Weekend days & $\begin{array}{c}11377 * \\
(\mathbf{9 , 9 3 0 - 1 2 , 8 2 4 )} \\
\end{array}$ & $\begin{array}{c}13,863 \\
(12,131-15,594)\end{array}$ \\
\hline \multicolumn{3}{|c|}{ \% exceeding MVPA criteria $(>12,000)$} \\
\hline All days & $36 \% *$ & $85 \%$ \\
\hline Weekdays & $27 \% *$ & $92 \%$ \\
\hline Weekend days & $44 \%$ & $65 \%$ \\
\hline
\end{tabular}

Note: mean $(95 \% \mathrm{CI})$ *sig. diff. from historical group; Gray indicates sig. diff. across days

Figure 60 
A.

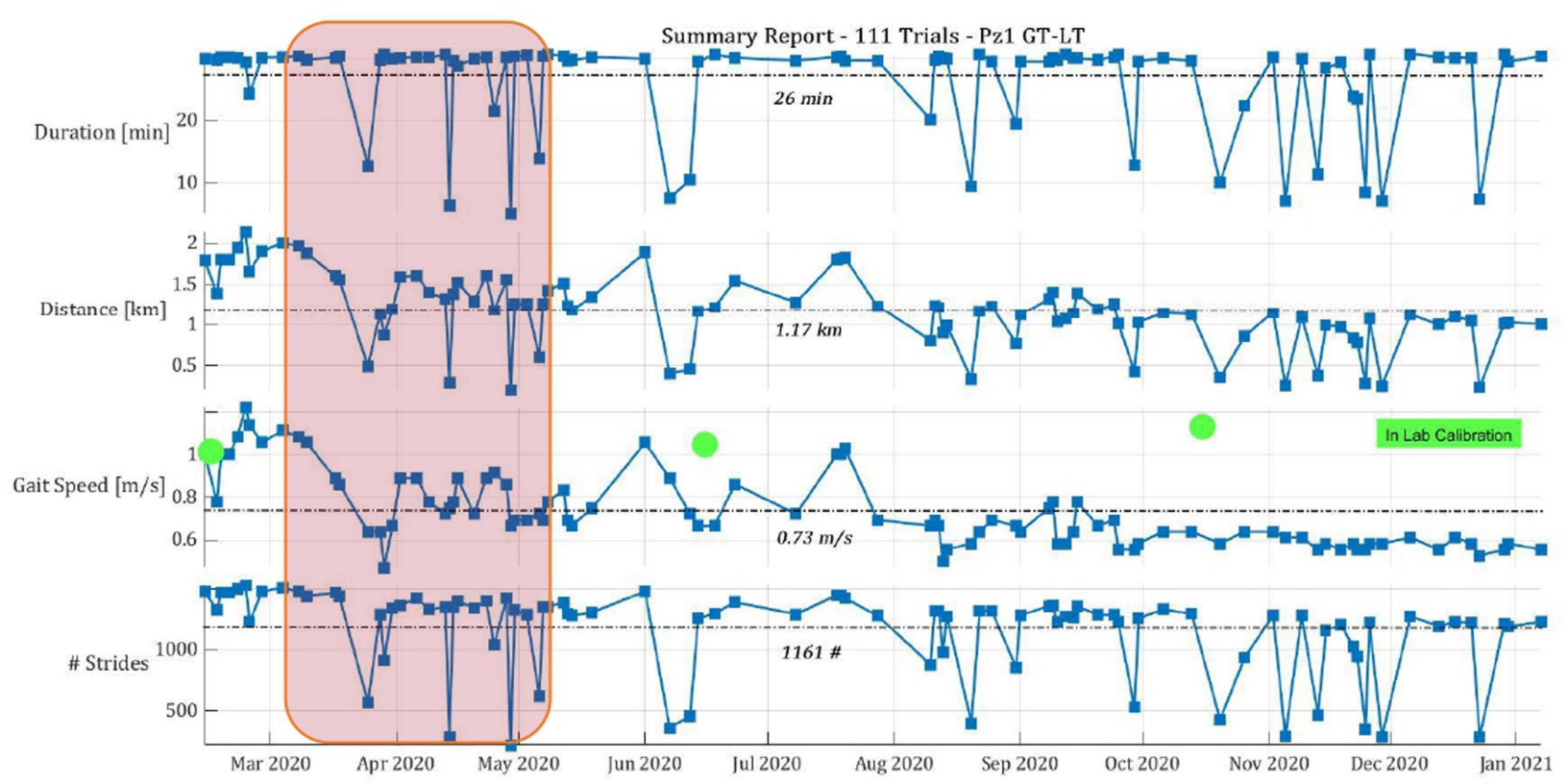

B.
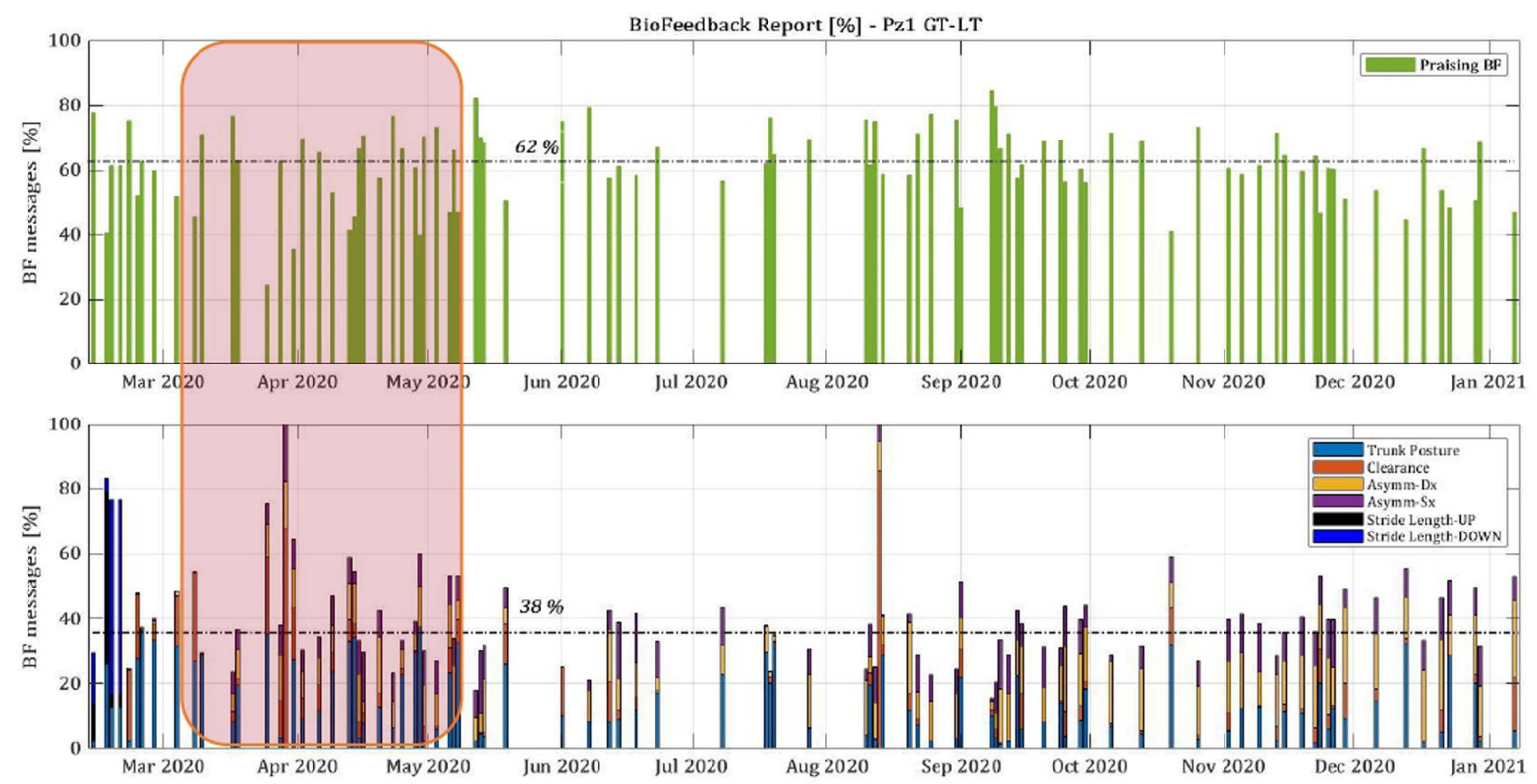

C.

\begin{tabular}{|c|c|c|c|c|c|c|c|c|c|c|}
\hline P1 & $\begin{array}{l}\text { MDS-UPDRS } \\
\text { Part III (on) }\end{array}$ & H\&Y & $\begin{array}{c}\text { BERG } \\
\text { Test }\end{array}$ & $\begin{array}{c}\text { MINI BEST } \\
\text { Test }\end{array}$ & $\begin{array}{l}\text { TUG } \\
{[s]}\end{array}$ & $\begin{array}{c}\text { TUG - Dual } \\
\text { Task [s] }\end{array}$ & $\begin{array}{c}\text { 10-WT } \\
{[\mathrm{s}]}\end{array}$ & MoCA & $\begin{array}{c}\text { Disease } \\
\text { Duration }\end{array}$ & Age \\
\hline TO_02/21/2020 & 29 & II & $55 / 56$ & $27 / 28$ & 11.81 & 15.25 & 5.50 & $25 / 30$ & 8 & 71 \\
\hline T1_07/17/2020 & 23 & II & $55 / 56$ & $25 / 28$ & 12.63 & 11.28 & 5.20 & 1 & 1 & 1 \\
\hline T2_10/16/2020 * & 23 & II & $54 / 56$ & $22 / 28$ & 11.56 & 12.24 & 5.02 & 1 & 1 & 1 \\
\hline T3_01/22/2021 & 24 & II & $53 / 56$ & $25 / 28$ & 10.52 & 11.34 & 5.30 & 1 & 1 & 1 \\
\hline \multicolumn{11}{|c|}{${ }^{\circ}$ The timing of clinical evaluations was modified for this patient due to the first lockdown for } \\
\hline
\end{tabular}

Fig. 1. A. Trend of the main outcomes achieved in each trial performed by P1: Duration time (26 [min]); Distance (1.17 [km]); Gait Speed $(0.73[\mathrm{~m} / \mathrm{s}])$ and Number of Strides (1161 [strides]). B. Percentage of Praising (62 [\%]) and Corrective biofeedback (38 [\%]) received in each trial. According to Clinician's indications, the main spatio-temporal gait parameters used as biofeedback variables by P1 were: Trunk Posture, Gait Asymmetry and Foot Clearance. The red box indicates the Italian lockdown period. C. Measures of progression of P1. H\&Y: Hoehn \& Yahr; TUG: Timed Up and Go; 10-WT: $10 \mathrm{mt}$ walking test; MoCA: Montreal Cognitive Assessment.

Figure 61 


\begin{tabular}{|c|c|c|c|c|c|c|c|c|c|c|c|}
\hline \multirow{2}{*}{ Variables } & \multicolumn{3}{|c|}{ Negative Ranks } & \multicolumn{3}{|c|}{ Positive ranks } & \multicolumn{3}{|c|}{ Test statistics } & \multirow{2}{*}{$\begin{array}{c}\text { Mean before } \\
\text { lockdown }\end{array}$} & \multirow{2}{*}{$\begin{array}{l}\text { Mean during } \\
\text { lockdown }\end{array}$} \\
\hline & $n$ & $\begin{array}{c}\text { Mean } \\
\text { rank }\end{array}$ & $\begin{array}{c}\text { Sum of } \\
\text { ranks }\end{array}$ & $n$ & $\begin{array}{c}\text { Mean } \\
\text { rank }\end{array}$ & $\begin{array}{c}\text { Sum of } \\
\text { ranks }\end{array}$ & Ties & z & $p$ & & \\
\hline $\begin{array}{l}\text { (Activity before lockdown) } \\
\text { - } \\
\text { (Activity during lockdown) }\end{array}$ & 120 & 89.99 & 10799 & 48 & 70.77 & 3397 & 390 & -6.139 & 0.000 & 4.19 & 3.98 \\
\hline $\begin{array}{l}\text { (General health before lockdown) } \\
\text { (General health during lockdown) }\end{array}$ & 14 & 45.86 & 642 & 83 & 49.53 & 4111 & 464 & -6.910 & 0.000 & 2.64 & 2.78 \\
\hline $\begin{array}{l}\text { (Mental health before lockdown) } \\
\text { (Mental health during lockdown) }\end{array}$ & 49 & 108.33 & 5308 & 264 & 166.03 & 43833 & 199 & -12.114 & 0.000 & 9.32 & 10.71 \\
\hline
\end{tabular}

Figure 62

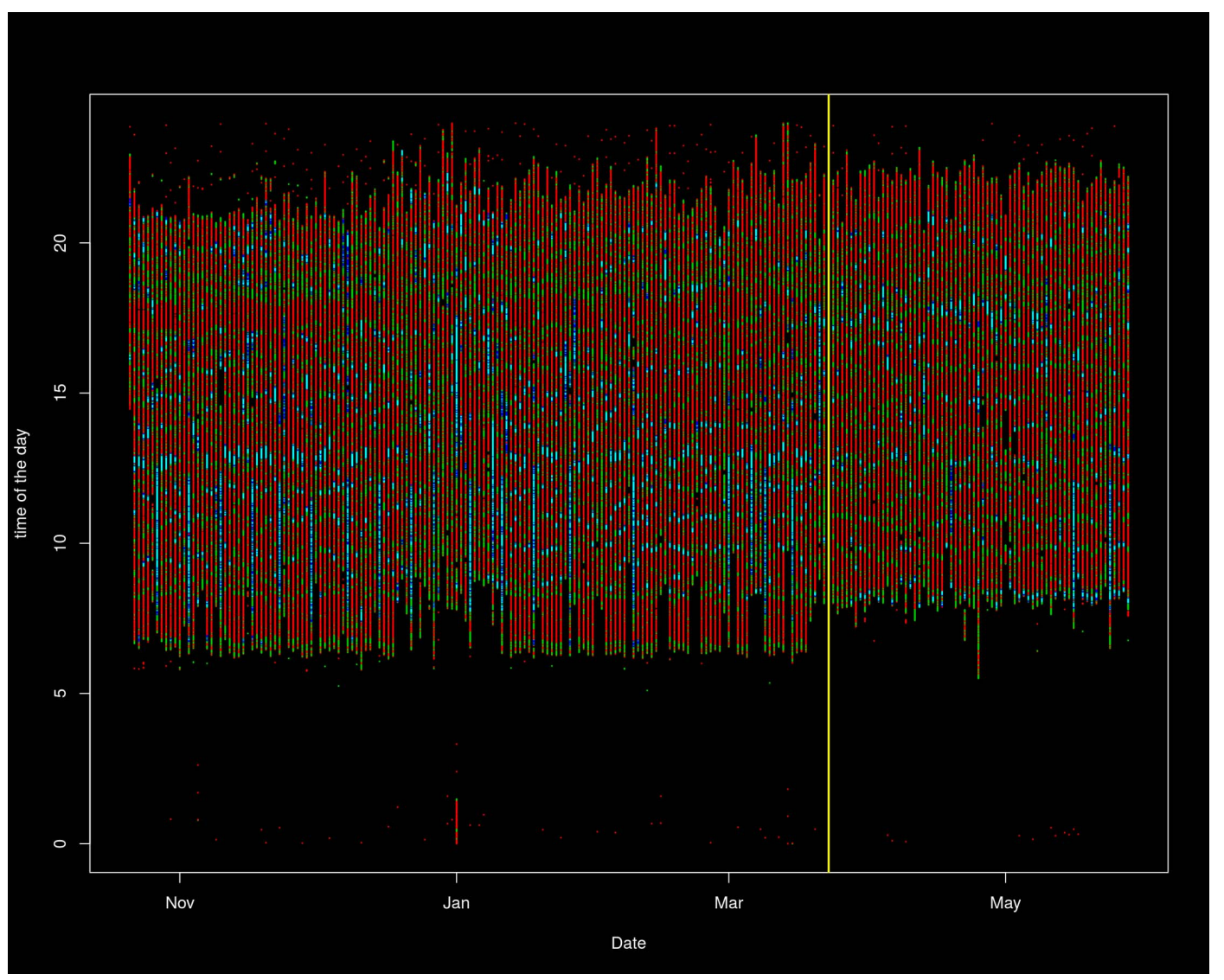

Figure 63 
Figure 1. The number of novel accelerometer methods included in the systematic review that were not made available, available within the paper as equations or cut-points, or are available

as code

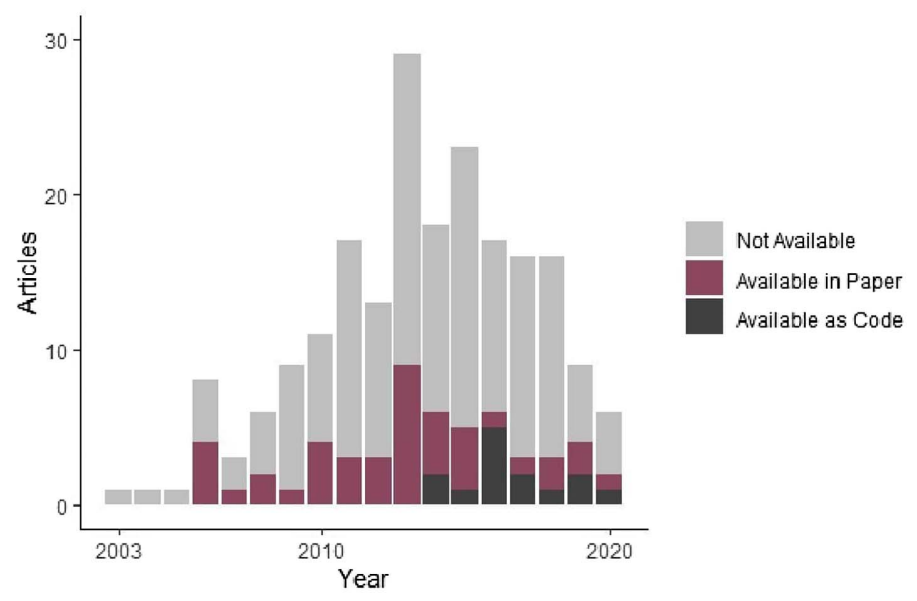

Figure 64

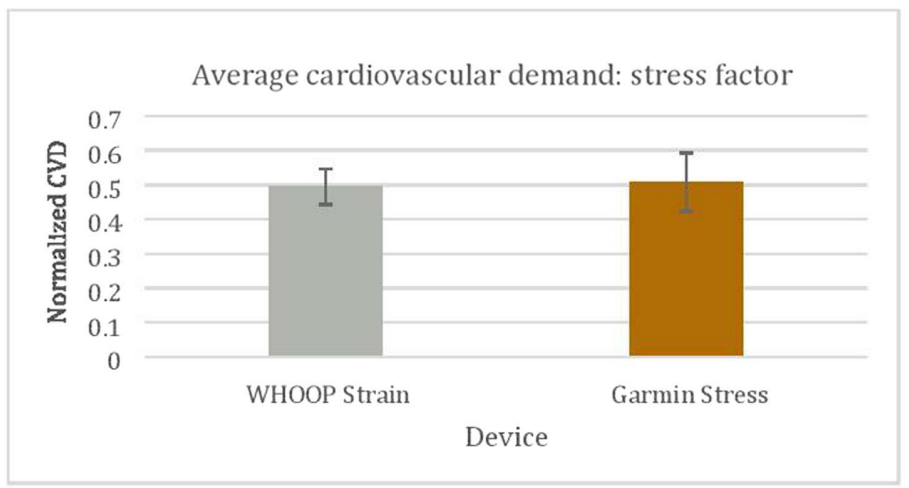

Figure 65

Figure 1. Average cardiovascular demand reported from respective devices $(\mathrm{N}=1)$

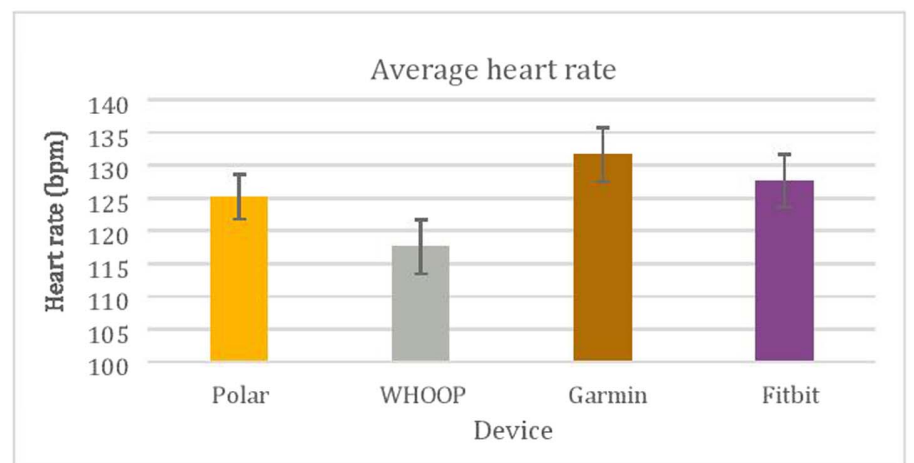

Figure 1. Average heart rate reported from devices compared to Polar H10

Figure 66 
(a) Linear ST

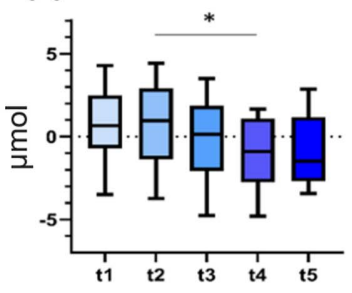

(b)

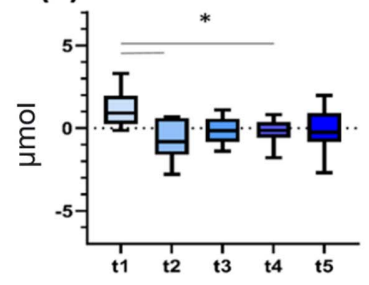

(c) F8WT Right ST

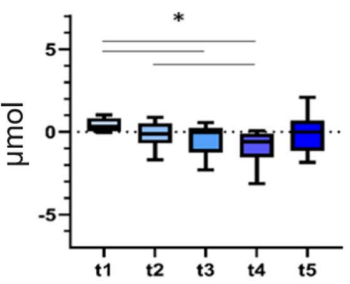

F8WT Right DT

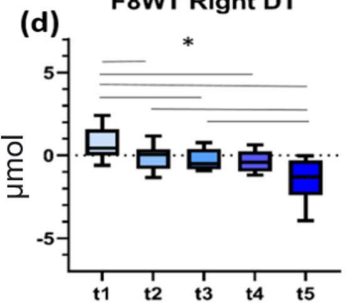

(e) F8WT Left ST
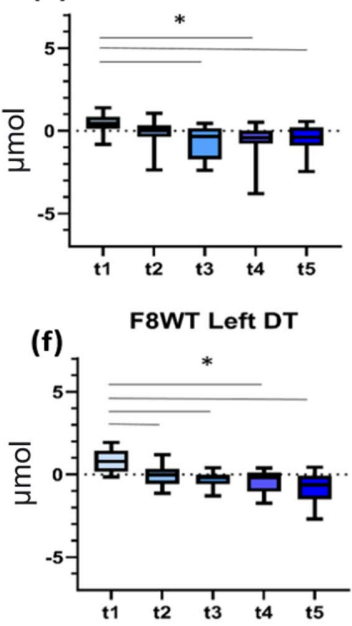

Figure 67 
Cross-sectional Associations between 24-hr Activity Behaviours and Cardiometabolic Health in Adolescents: A Compositional Data Analysis

G. Hayes $^{1}$, L. D. Browne ${ }^{6}$, K. P. Dowd (Ph.D.) $)^{2}$, C. MacDonncha (Ph.D. $)^{1,3}$, B.P. Carson (Ph.D.) $)^{1,3}$, H. Purtill (Ph.D.) $)^{3,4}$, A. Hannigan (Ph.D.) ${ }^{3}$, M. P. Herring (Ph.D.) ${ }^{1,3}$, E. M. O' Connor (Ph.D. $)^{3,5}$, C. S. O' Gorman (MD) ${ }^{3,6}$, and A. E. Donnelly (Ph.D. $)^{1.3}$.

${ }^{1}$ Department of Physical Education and Sport Sciences, University of Limerick, Limerick, Ireland,

${ }^{2}$ Department of Sport and Health Sciences, Athlone Institute of Technology, Westmeath, Ireland

${ }^{3}$ Health Research Institute, University of Limerick, Limerick, Ireland

${ }^{4}$ Department of Maths and Statistics, University of Limerick, Limerick, Ireland,

${ }^{5}$ Department of Biological Sciences, University of Limerick, Limerick, Ireland.

${ }^{6}$ School of Medicine, University of Limerick, Limerick, Ireland.

Funding: This study was funded by a grant from the Health Research Institute, University of Limerick, Ireland.

Table 1. Estimated mean predicted change (95\%) in health indicators related to pairwise reallocations of 30 minutes of sleep, sedentary time, standing to LIPA (Part A) or MVPA (Part B).

\begin{tabular}{|c|c|c|c|}
\hline Part A & Sleep to LIPA & SedT to LIPA & Standing to LIPA \\
\hline Health Indicator & $\begin{array}{l}\text { Predicted Change } \\
(95 \% \text { CI) }\end{array}$ & $\begin{array}{l}\text { Predicted Change } \\
(95 \% \text { CI) }\end{array}$ & $\begin{array}{l}\text { Predicted Change } \\
(95 \% \text { CI) }\end{array}$ \\
\hline BMI kg.m² & $-0.75(-1.59,0.1)$ & $-0.78(-1.58,0.02)$ & $-1.07(-2.08,-0.07)^{*}$ \\
\hline Sum of Skinfold thickness (mm) & $-4.94(-9.42,-0.46)^{*}$ & $-4.57(-8.81,-0.33)^{*}$ & $-5.94(-11.27,-0.62)^{*}$ \\
\hline WHR & $0.00(-0.01,0.01)$ & $0.00(-0.01,0.01)$ & $0.00(-0.01,0.01)$ \\
\hline Grip strength $(\mathrm{Kg})$ & $0.43(-0.84,1.7)$ & $0.80(-0.41,2)$ & $0.26(-1.25,1.77)$ \\
\hline $\begin{array}{l}\text { Cardiorespiratory Fitness } \\
\left(\mathrm{ml} / \mathrm{kg} / \mathrm{min}^{-1}\right)\end{array}$ & $1.58(0.23,2.94) *$ & $1.57(0.31,2.83)^{*}$ & $1.41(-0.20,3.02)$ \\
\hline Systolic BP (mmHg) & $-0.14(-2.92,2.65)$ & $0.55(-2.1,3.19)$ & $0.56(-2.76,3.88)$ \\
\hline Diastolic BP ((mmhg & $-3(-4.98,-1.03)^{*}$ & $-3.15(-5.02,-1.28)^{*}$ & $-2.35(-4.7,0)$ \\
\hline Total Cholesterol (mmol/L) & $0.04(-0.12,0.2)$ & $0.02(-0.14,0.18)$ & $0.10(-0.1,0.29)$ \\
\hline HDL_C $(\mathrm{mmol} / \mathrm{L})$ & $0.01(-0.04,0.06)$ & $-0.01(-0.06,0.05)$ & $-0.01(-0.07,0.05)$ \\
\hline LDL-C (mmol/L) & $0.04(-0.09,0.18)$ & $0.03(-0.1,0.15)$ & $0.10(-0.06,0.26)$ \\
\hline Triglycerides (mmol/L) & $0.00(-0.07,0.07)$ & $0.00(-0.06,0.07)$ & $0.03(-0.06,0.11)$ \\
\hline Glucose $(\mathrm{mmol} / \mathrm{L})$ & $-0.03(-0.10,-0.05)$ & $-0.05(-0.12,0.03)$ & $-0.04(-0.13,0.05)$ \\
\hline Part B & Sleep to MVPA & SedT to MVPA & Standing to MVPA \\
\hline Health Indicator & $\begin{array}{l}\text { Predicted Change } \\
(95 \% \text { CI })\end{array}$ & $\begin{array}{l}\text { Predicted Change } \\
\text { (95\% CI) }\end{array}$ & $\begin{array}{l}\text { Predicted Change } \\
(95 \% \text { CI) }\end{array}$ \\
\hline BMI (Kg.m²) & $0.55(0.06,1.04) *$ & $0.51(0.07,0.95)^{*}$ & $0.22(-0.36,0.8)$ \\
\hline Sum of Skinfolds (mm) & $1.91(-0.68,4.49)$ & $2.28(-0.05,4.62)$ & $0.90(-2.15,3.96)$ \\
\hline WHR & $0(0,0.01)$ & $0.00(0.00,0.01)$ & $0.00(0,0.01)$ \\
\hline Grip strength $(\mathrm{Kg})$ & $-0.25(-0.99,0.49)$ & $0.11(-0.55,0.78)$ & $-0.42(-1.28,0.44)$ \\
\hline $\begin{array}{l}\text { Cardiorespiratory Fitness } \\
\left(\mathrm{ml} / \mathrm{kg} / \mathrm{min}^{-1}\right)\end{array}$ & $0.55(-0.02,1.3)$ & $0.53(-0.13,1.2)$ & $0.37(-0.52,1.25)$ \\
\hline Systolic BP (mmHg) & $-1.80(-3.42,-0.19) *$ & $-1.12(-2.58,0.34)$ & $-1.10(-3,0.79)$ \\
\hline Diastolic BP (mmHg) & $0.23(-0.91,1.37)$ & $0.08(-0.95,1.12)$ & $0.88(-0.46,2.22)$ \\
\hline Total Cholesterol (mmol/L) & $-0.03(-0.12,0.07)$ & $-0.05(-0.13,0.04)$ & $0.03(-0.08,0.14)$ \\
\hline HDL-C (mmol/L) & $0.04(0.01,0.07)^{*}$ & $0.03(0.00,0.05)$ & $0.02(-0.02,0.06)$ \\
\hline LDL-C (mmol/L) & $-0.04(-0.12,0.04)$ & $-0.05(-0.12,0.02)$ & $0.02(-0.07,0.11)$ \\
\hline Triglycerides (mmol/L) & $-0.06(-0.1,-0.01)^{*}$ & $-0.05(-0.09,-0.01) *$ & $-0.03(-0.07,0.02)$ \\
\hline Glucose (mmol/L) & $0.00(-0.05,0.04)$ & $-0.02(-0.06,0.02)$ & $-0.01(-0.07,-0.04)$ \\
\hline
\end{tabular}

Figure 68 


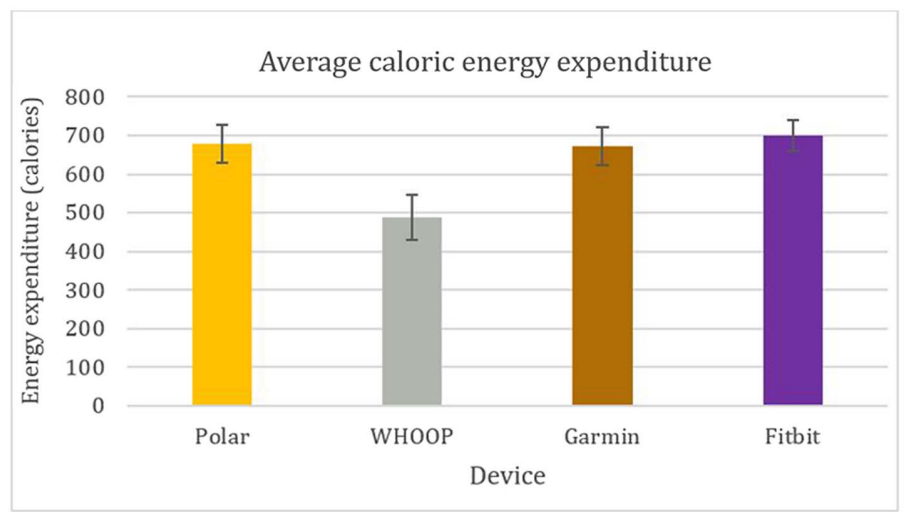

Figure 1. Average energy expenditure reported from devices

Figure 69

Participated in the laboratory measurements: invited to accelerometry $n=910$

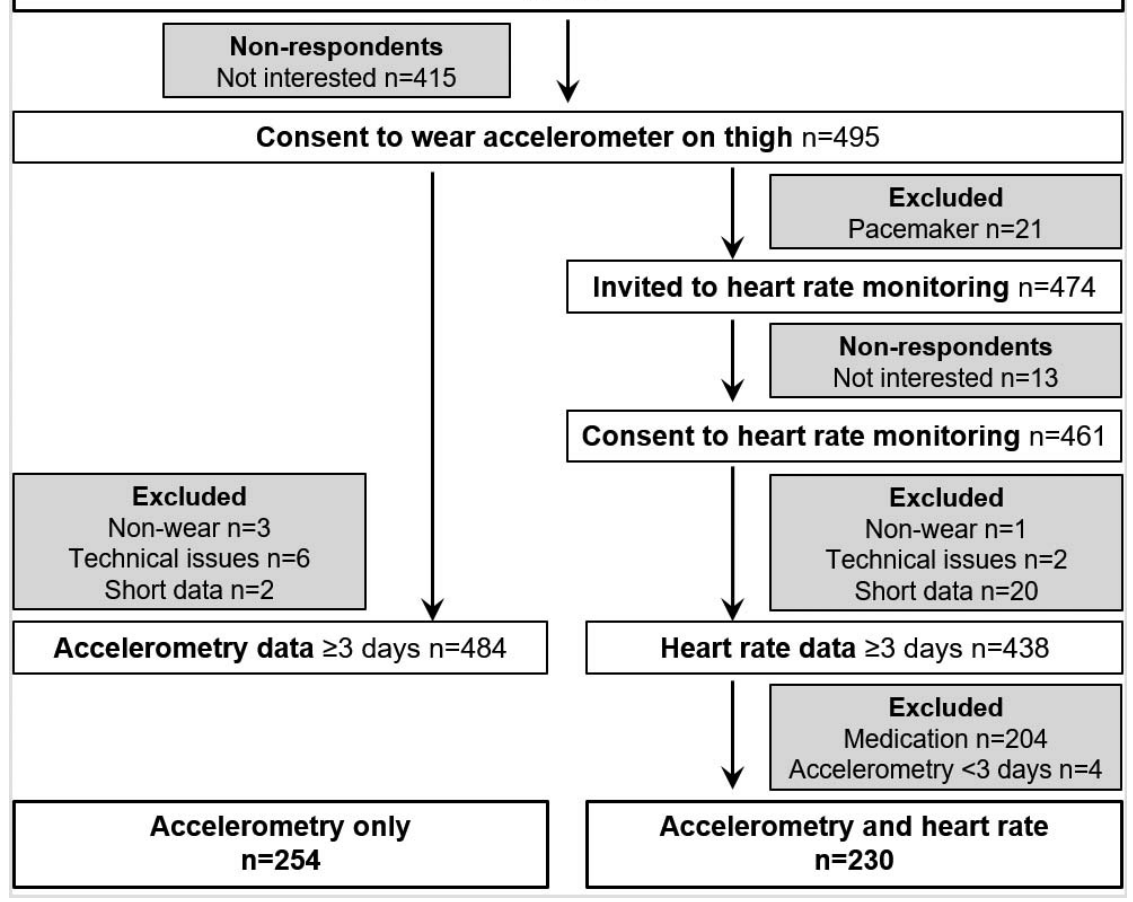

Figure 70 
Table 1: Longitudinal associations of different physical activity patterns with physical function after adjusting for covariates

\begin{tabular}{|c|c|c|c|c|}
\hline Variables & $\begin{array}{l}\text { Unstandardized } \\
\beta \text { Coefficients }\end{array}$ & $\begin{array}{l}\text { Standardized } \\
\beta \text { Coefficients }\end{array}$ & $95 \% \mathrm{Cl}$ & $\mathbf{p}$ \\
\hline \ 2-Minute Walk Test (metres) & & & & \\
\hline$\Delta$ PAFrag using $\geq 120$-second PA bouts (\%) & -0.21 & -0.06 & -0.46 to 0.05 & 0.111 \\
\hline$\Delta$ PAFrag using $\geq 300$-second PA bouts $(\%)^{a}$ & -0.55 & -0.09 & -0.98 to -0.12 & 0.013 \\
\hline
\end{tabular}

Abbreviations: $\boldsymbol{\Lambda}$ = change at follow-up minus baseline; $95 \% \mathrm{Cl}=95 \%$ confidence intervals; $\mathrm{PA}=$ physical activity; PAFrag = physical activity fragmentation

${ }^{a} n=649$ due to 41 participants not completing any 300 -second physical activity bouts

Note: Both models were adjusted for daily sedentary behaviour time, daily wear-time, country, sex, age, body mass index, education, number of comorbidities, physical SF-12, mental SF-12 and twominute walk test distance (all measured at baseline).

Figure 71

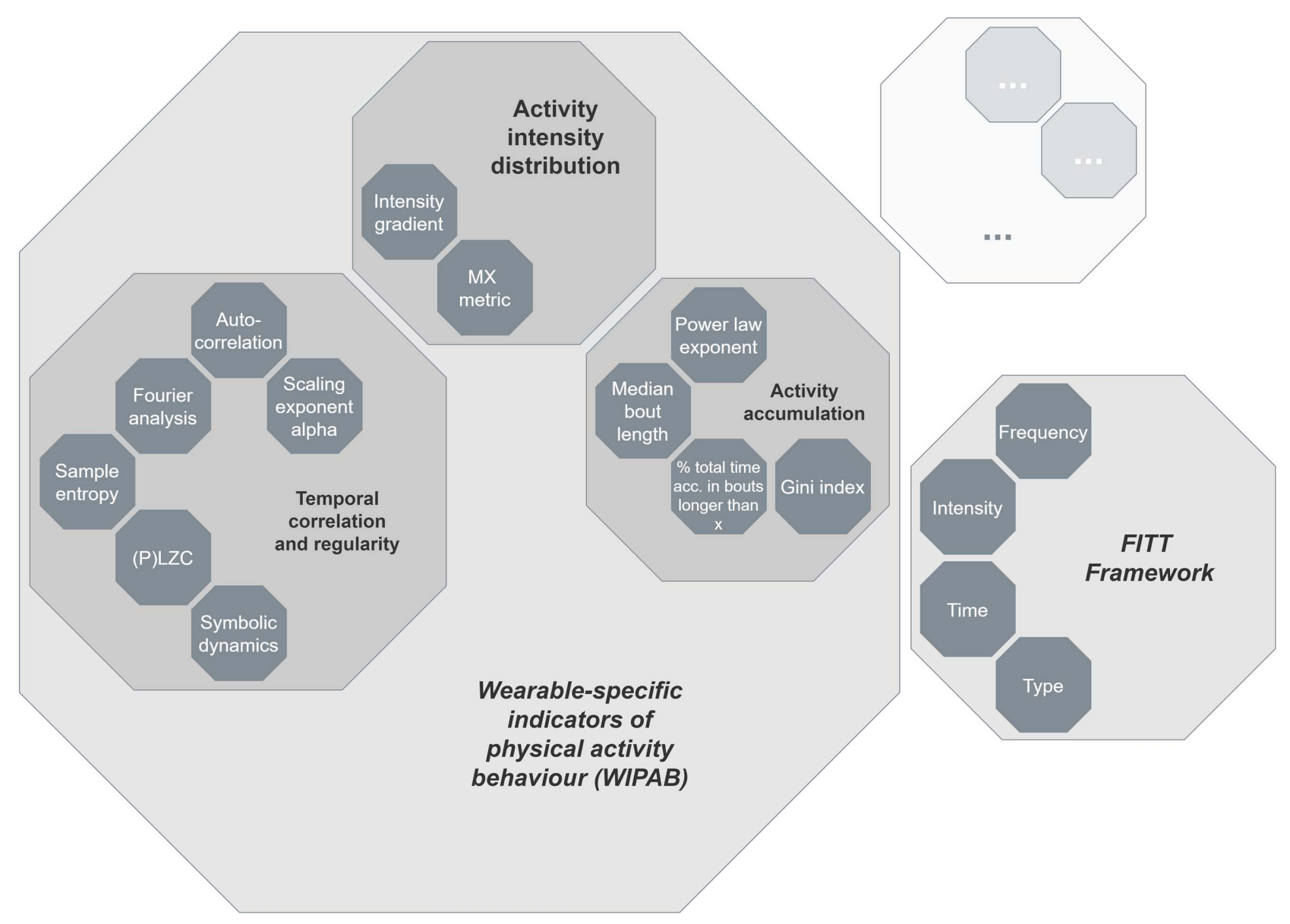

Figure 72 